\title{
Cardiac troponins
}

Citation for published version (APA):

van der Linden, N. (2018). Cardiac troponins: State of the (He)Art; towards optimization of interpretation. [Doctoral Thesis, Maastricht University]. Maastricht University. https://doi.org/10.26481/dis.20180119nvdl

\section{Document status and date:}

Published: 01/01/2018

DOI:

10.26481/dis.20180119nvdl

Document Version:

Publisher's PDF, also known as Version of record

\section{Please check the document version of this publication:}

- A submitted manuscript is the version of the article upon submission and before peer-review. There can be important differences between the submitted version and the official published version of record.

People interested in the research are advised to contact the author for the final version of the publication, or visit the DOI to the publisher's website.

- The final author version and the galley proof are versions of the publication after peer review.

- The final published version features the final layout of the paper including the volume, issue and page numbers.

Link to publication

\footnotetext{
General rights rights.

- You may freely distribute the URL identifying the publication in the public portal. please follow below link for the End User Agreement:

www.umlib.nl/taverne-license

Take down policy

If you believe that this document breaches copyright please contact us at:

repository@maastrichtuniversity.nl

providing details and we will investigate your claim.
}

Copyright and moral rights for the publications made accessible in the public portal are retained by the authors and/or other copyright owners and it is a condition of accessing publications that users recognise and abide by the legal requirements associated with these

- Users may download and print one copy of any publication from the public portal for the purpose of private study or research.

- You may not further distribute the material or use it for any profit-making activity or commercial gain

If the publication is distributed under the terms of Article $25 \mathrm{fa}$ of the Dutch Copyright Act, indicated by the "Taverne" license above, 
Cardiac troponins: State of the (He)Art towards optimization of interpretation 
Cardiac troponins: State of the (He)Art - towards optimization of interpretation Noreen van der Linden

\section{ISBN 9789462957848}

Cover illustration by Puck Rouvroye

Layout by Noreen van der Linden and Puck Rouvroye

Printed by ProefschriftMaken || www.proefschriftmaken.nl

(c) Copyright Noreen van der Linden, Rotterdam 2017 


\title{
Cardiac troponins: State of the (He)Art
}

\author{
towards optimization of interpretation
}

\section{PROEFSCHRIFT}

ter verkrijging van de graad van doctor aan de Universiteit Maastricht, op gezag van de Rector Magnificus, Prof. dr. Rianne M. Letschert, volgens het besluit van het College van Decanen, in het openbaar te verdedigen op vrijdag 19 januari 2018 om 14.00 uur

door

Noreen van der Linden 


\section{Promotores}

Prof. dr. M.P. van Dieijen-Visser

Prof. dr. L.J.C. van Loon

\section{Copromotor}

Dr. S.J.R. Meex

\section{Beoordelingscommissie}

Prof. dr. H.J.G.M. Crijns (voorzitter/chair)

Prof. dr. E. Giannitsis (Universitätsklinikum Heidelberg, Heidelberg, Germany)

Prof. dr. T.M. Hackeng

Prof. dr. Y.M. Pinto (Academisch Medisch Centrum, Amsterdam)

Prof. dr. C.D.A. Stehouwer

The research described in this thesis was supported by a grant of Stichting de Weijerhorst.

Financial support by Sysmex for the publication of this thesis is greatly acknowledged.

Financial support by the Dutch Heart Foundation for the publication of this thesis is greatly acknowledged. 


\section{Contents}

Chapter $1 \quad$ General introduction 7

Chapter $2 \quad$ Strong diurnal rhythm of troponin T, but not troponin I, in a patient 21 with renal dysfunction

Chapter 3 Diurnal rhythm of cardiac troponin: consequences for the diagnosis of acute myocardial infarction

Chapter $4 \quad$ Twenty-four-hour biological variation profiles of cardiac troponin I in subjects with or without chronic kidney disease

Chapter $5 \quad$ Origin of cardiac troponin $\mathrm{T}$ in chronic kidney disease

Chapter $6 \quad$ Mass spectrometric identification of cardiac troponin T in urine of patients suffering from acute myocardial infarction

Chapter $7 \quad$ Large variation in measured cardiac troponin T concentrations after standard addition in serum or plasma of different individuals

Chapter $8 \quad$ Combining cardiac troponin I and cardiac troponin T for the early diagnosis of acute myocardial infarction

Chapter 9 Effect of a six-month resistance-type exercise training program on 151 the course of cardiac troponin T levels in (pre)frail elderly

Chapter 10 Effect of exercise training on the course of cardiac troponin $\mathrm{T}$ and I 157 levels: three independent training studies

Chapter 11 Prognostic value of basal cardiac troponin levels on mortality in the 173 general population

Chapter 12 General discussion

Supplemental Summary, Samenvatting $\quad 207$

Valorization 217

Dankwoord, Curriculum Vitae, List of publications 221 

General introduction 


\section{Acute myocardial infarction: a major burden of morbidity and mortality}

Despite the decreasing prevalence of cardiovascular disease in the Western World ${ }^{1}$, acute myocardial infarction (also known as heart attack) remains an important burden of morbidity and mortality worldwide ${ }^{2}$. In the Netherlands, with approximately 17 million inhabitants, each year 70000 people are diagnosed with acute myocardial infarction ${ }^{3,4}$. This etiology substantially contributes to mortality-acute myocardial infarction is considered as the cause of death in approximately 5000 people yearly-, morbidity and related public health expenses ${ }^{3,5-7}$.

Chest pain, an important indicator of acute myocardial infarction, is reported by approximately $10 \%$ of all subjects visiting the emergency department ${ }^{8}$, a population of which 15-20\% does actually have an acute myocardial infarction ${ }^{9}$. Correct and rapid distinction of subjects with and without myocardial infarction assists the allocation of treatment to those who need it and prevents unnecessary resource use. Therefore, accurate diagnostics may, besides prevention and improved treatment, contribute to lower the burden of acute myocardial infarction ${ }^{9-16}$.

\section{The perfect biomarker for the diagnosis of acute myocardial infarction}

The diagnosis of acute myocardial infarction is based on the presence of characteristic clinical symptoms, like chest pain, accompanied by electrocardiogram (ECG) changes or by elevated levels of cardiac proteins (nowadays cardiac troponins) ${ }^{17,18}$. Whereas deviations on the $12-$ lead ECG are only clearly present in 30-40\% of patients with a myocardial infarction ${ }^{19-22}$, cardiac biomarkers in the blood play an important role in the diagnosis of acute myocardial infarction. During the last decades, an evolution in biomarkers used for the diagnosis has taken place. Subsequently, aspartate aminotransferase (AST), lactate dehydrogenase (LDH), creatine kinase (CK), creatine kinase $M B$ isoenzyme (CK-MB), and cardiac troponins were the biomarkers used in a clinical setting to detect acute myocardial infarction ${ }^{23}$. Time and again, a biomarker was replaced by another marker with more 'desirable characteristics ${ }^{24-26}$. Table 1 shows an overview of biomarker characteristics that are considered to be important ${ }^{26,27}$. 


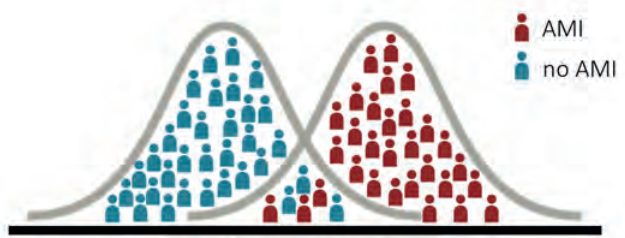

Biomarker concentration $\rightarrow$

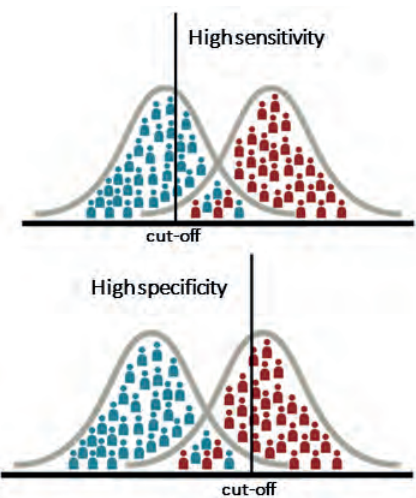

Figure 1. Sensitivity and specificity depend on the cut-off concentration. If there is an overlap in biomarker concentrations of people with and without a certain disease, it is not possible to have a test with a $100 \%$ sensitivity and a $100 \%$ specificity. In this case a higher sensitivity will be accompanied by a lower specificity, and vice versa. A cut-off concentration with a high sensitivity is suitable for the safe rule-out, and one with a high-specificity for the rule-in of subjects.

In general, the preferred characteristics of cardiac biomarkers strongly depend on their intended use $^{28}$. In case of the diagnosis of acute myocardial infarction, its accurate recognition - the allocation of subjects in a group with and without this specific etiology - is the main goal. Therefore, an important aspect for the evaluation of a biomarker for the diagnosis of acute myocardial infarction is the accuracy: The amount of agreement between the results of the biomarker test under evaluation (index test) and the gold standard diagnosis 29,30 . The accuracy is often represented by the sensitivity (detection of truepositives: a positive result when the disease is truly present) and specificity (detection of true negatives: a negative result when the disease is truly absent) at a selected cut-off point ${ }^{27}$, or by the area under a receiver operating characteristic (ROC) curve ${ }^{30}$. Hypothetically, the ideal test makes the perfect distinction between subjects with and without myocardial infarction, and should, therefore, present both a maximum, 100\%, sensitivity and specificity. However, in reality, there is often an overlapping range in biomarker concentrations between subjects with and without disease. Hence, the definition of a cut-off point is required. This causes a high sensitivity (almost all subjects that truly have an acute myocardial infarction have a positive test result) at the expense of lower specificity (a high number of false positive test results), or the other way around (Figure 1 ). 
Table 1. Desirable characteristics of biomarkers for the diagnosis of acute myocardial infarction ${ }^{26,27}$

\begin{tabular}{ll}
\hline General features & Acceptable to the patient \\
& Add to clinical assessment \\
& Ability to influence therapy \\
& Ability to improve patient outcome \\
& Cost-effective \\
& Applicable to (almost) all patients, always \\
& High myocardial specificity \\
& Release proportional to injury size or ischemic burden \\
& Suitable for early diagnosis: immediate release \\
& Permit long time window for diagnosis \\
& Permit diagnosis of recurrent ischemia \\
\hline Test features & Ability to detect even small infarctions \\
& No special sample preparation needed \\
& Rapid test / Short turnaround time \\
& Test readily available \\
& Easy interpretable \\
& Single measure representative \\
& Internationally standardized \\
High accuracy \\
High precision \\
Compatible with existing logistics \\
\hline Assay / measurement features
\end{tabular}

\section{Cardiac troponins as biomarkers for acute myocardial infarction}

According to the universal definition, acute myocardial infarction is cardiomyocyte death due to prolonged myocardial ischemia, an imbalance between oxygen demand and supply ${ }^{17}$. As a result of this process of ischemic injury and subsequent cell necrosis, cardiomyocyte content is released into the peripheral blood stream. After a finite period of time, cardiac ischemia will be reflected by increasing concentrations of several biomarkers, like cardiac troponins, in the peripheral blood ${ }^{31,32}$.

Cardiac troponins form part of the intracellular content of cardiomyocytes. The cardiac troponin complex, consisting of a troponin $\mathrm{T}, \mathrm{I}$ and $\mathrm{C}$ protein, fulfills a key role in cardiomyocyte contraction by regulation of the binding between the actin and the myosin filaments. In general, troponins are structurally bound to the actin filaments of the contractile apparatus of the cardiomyocytes ${ }^{33}$. In addition, it has been suggested that a smaller portion (3-8 \%) may be present as unbound protein in the cytosol ${ }^{34-36}$. Two proteins of the cardiac troponin complex, cardiac troponin T (cTnT) and cardiac troponin I (cTnl), are 
exclusively expressed in the heart, and are therefore considered as cardiac specific biomarkers $33,37-39$.

Healthy cardiomyocyte

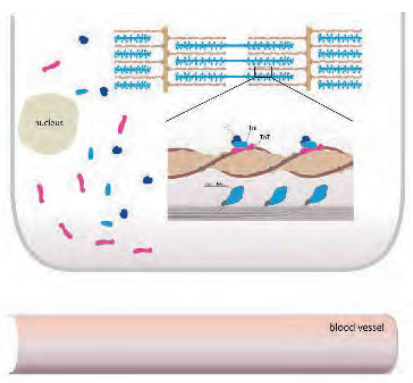

$\underline{\text { Ischemic cardiomyocyte }}$

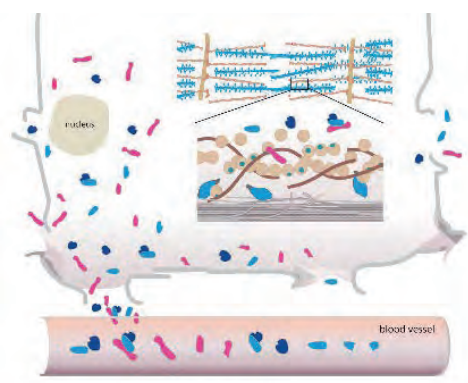

Figure 2. Release of cardiac troponins following acute myocardial infarction. In the healthy heart, cardiac troponins are limited to the cardiomyocytes where they play an essential role in muscle contraction coupling ${ }^{33}$. In cardiac ischemia, altered cell membrane integrity causes release of troponin into the circulation as the contractile apparatus breaks down 32,35 .

\section{The detection of cardiac troponins: from conventional to high-sensitivity assays}

The discovery of cardiac specific troponins triggered the interest in cardiac troponins as cardiac specific biomarkers. The first immunoassay for the detection of cTnl in serum was developed in $1987^{40}$, followed by the first CTnT assay in $1989^{41}$. These initial assays were characterized by several 'undesirable properties' such as an extended turn-around time, low analytical sensitivity and cross-reactivity with skeletal muscle troponins ${ }^{23}$. In the subsequent years, the continuous evolution in assays led to improvement of cardiac specificity, analytical sensitivity, and assay precision at the low measurement range $23,42-45$. In 2010, the first highsensitivity assay was implemented and in the following years multiple high-sensitivity assays became commercially available and are now widely used in most Western European countries $^{46,47}$. A troponin assay can be considered as a high-sensitivity assay if at least $50 \%$ of the general population exhibits measurable levels below the $99^{\text {th }}$ percentile $e^{48-52}$.

As shown in table 2, there are multiple conventional and high-sensitivity cTnl assays in the marketplace and, due to intellectual property rights, there is only one manufacturer that produces cTnT assays. Because cTnT and cTnl are different proteins with distinct 
characteristics, their measured concentrations will differ in individuals ${ }^{33,53,54}$. Nevertheless, it is important to consider that this is also the case for cTnl measured with different assays since the assays are not standardized and have various combinations of antibodies, incubation conditions, and detection technology 49 . As a result, the guidelines on the diagnosis of acute myocardial infarction use different, assay-specific cut-off values ${ }^{18,55}$.

Table 2. Analytical characteristics of the troponin assays used throughout this thesis ${ }^{49,50,52}$

\begin{tabular}{|c|c|c|c|c|c|}
\hline Assay & Manufacturer & $\mathrm{LoB}^{\mathrm{a}}, \mathrm{ng} / \mathrm{L}$ & LoD $^{b}, \mathrm{ng} / \mathrm{L}$ & $99^{\text {th }}$ percentile, $\mathrm{ng} / \mathrm{L}$ & CV at $99^{\text {th }}$ percentile, $\%$ \\
\hline \multicolumn{6}{|c|}{ Conventionalassays } \\
\hline cTnl & Abbott & NA & 9 & 28 & 14 \\
\hline cTnl & Beckman Coultier & $<10$ & 10 & 40 & 10 \\
\hline cTnT & Roche & 10 & NA & $<10$ & NA \\
\hline \multicolumn{6}{|c|}{ High-sensitivity assays } \\
\hline hs-cTnl & Abbott & $0.7-1.3$ & $1.1-1.9$ & 26.2 & $4.0-6.0$ \\
\hline hs-cTnl & Siemens & NA & 0.5 & 9 & $<10$ \\
\hline hs-cTnl & Singulex & NA & 0.09 & 10.1 & 9.0 \\
\hline hs-cTnT & Roche & 3 & 5 & 14 & 8.0 \\
\hline
\end{tabular}

aThe highest apparent analyte concentration expected to be found when replicates of a sample containing no analyte are tested. bLowest analyte concentration likely to be reliably distinguished from the LoB and at which detection is feasible ${ }^{56}$.

\section{The interpretation of cardiac troponins in the era of high-sensitivity assays}

In the year 2000, before the introduction of the high-sensitivity assays, cardiac troponins were designated as the preferred biomarkers for the diagnosis of acute myocardial infarction ${ }^{17,57-60}$. According to the first consensus document, a maximal concentration of cTnT or cTnl exceeding the decision limit $\left(99^{\text {th }}\right.$ percentile upper reference limit of a healthy reference population) on at least one occasion during the first 24 hours after the index clinical event was considered as a biochemical indicator for myocardial injury ${ }^{57}$. To be more specific in the detection of acute coronary events, the interpretation of cardiac troponins was further specified as a dynamic elevation, rise and/or fall, of cardiac troponin with at least one value above the $99^{\text {th }}$ percentile (if compatible with the clinical presentation) ${ }^{50,58}$. This definition of acute myocardial infarction remained the same after the introduction of the high-sensitivity assays, with the proviso that the increased sensitivity of the new assays 
allowed a shorter time interval between two consecutive cardiac troponin assessments (three instead of six hours), and hence allows a faster diagnosis ${ }^{9,61-64}$.
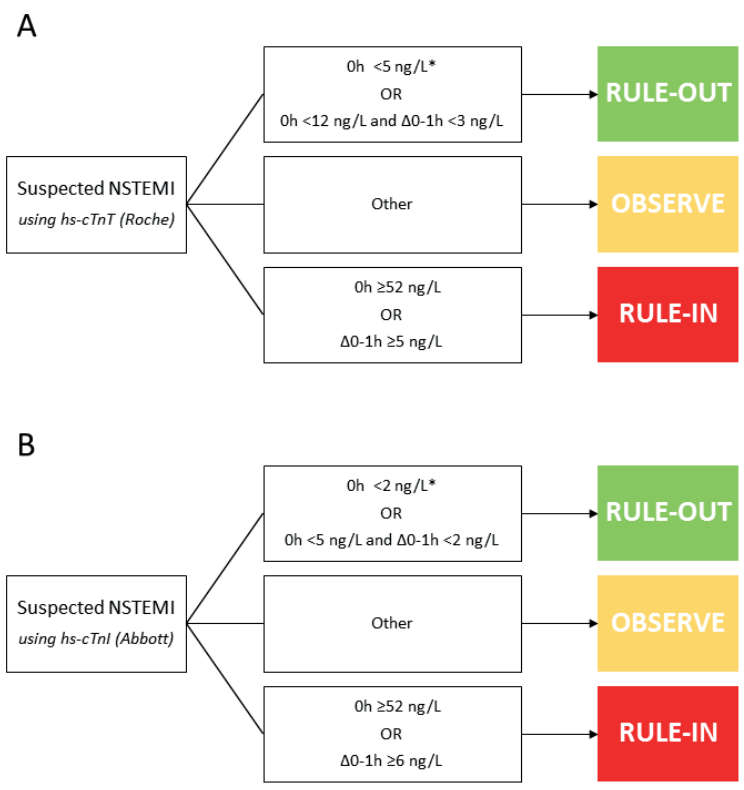

Figure $3.0 \mathrm{~h} / 1 \mathrm{~h}$ rule-in and rule-out algorithm. Based on assays-specific cut-off values acute myocardial infarction can be rapidly ruled-in and ruled-out at presentation or within one hour. Patients who do not qualify for 'rule-out' or 'rule-in' require a further high-sensitivity cardiac troponin assessment (e.g. at 3 h) ${ }^{18}$. ${ }^{*}$ Only applicable if chest pain onset $>3 h$

In 2015, the European Society of Cardiology presented its new guidelines with two alternative algorithms for the diagnosis of acute myocardial infarction based on highsensitivity cardiac troponins; a $0 \mathrm{~h} / 3 \mathrm{~h}$ algorithm and a $0 \mathrm{~h} / 1 \mathrm{~h}$ algorithm. Whereas the interpretation of cardiac troponin levels in the $0 \mathrm{~h} / 3 \mathrm{~h}$ algorithm is not substantially different from that of the previous $0 \mathrm{~h} / 6 \mathrm{~h}$ algorithm (one value above the $99^{\text {th }}$ percentile and a rise/fall defined by a delta-change-value), the $0 \mathrm{~h} / 1 \mathrm{~h}$ algorithm is based on a different approach (Figure 3$)^{18,65-70}$. 


\section{The expense and opportunities of high-sensitivity cardiac troponin assays}

The ability to measure previously undetectable concentrations of cardiac troponins expedited the clinical use of lower cut-off levels and significantly increased the sensitivity to detect (small) acute myocardial infarctions ${ }^{9,12}$. However, the increase in sensitivity due to lower cut-off values came at the expense of a decreased specificity ${ }^{12}$ : Elevations of cardiac troponin levels turned out not to be specific for acute myocardial infarction, but were also associated with several other acute and chronic conditions like myocarditis, heart failure, pulmonary embolism, stroke, stable coronary artery disease, type 2 diabetes mellitus, decreased renal function and as the result of prolonged exercise ${ }^{32,71-80}$. Since the cut-off values of the current diagnostic algorithms are specifically aimed at not missing subjects with acute myocardial infarction (thus the sensitivity is rather high), this may lead to an overdiagnosis or even an overtreatment of patients with persistently elevated cardiac troponin concentrations ${ }^{81,82}$. Therefore, the further optimization of interpretation of cardiac troponin concentrations in diagnosis seems to be indicated and prompts questions like: How may these conditions affect the diagnosis of acute myocardial infarction? And, how can diagnostic algorithms, or even assays, be adjusted for these effects?

Notwithstanding, the introduction of the high-sensitivity assays also facilitated the exploration of basal cardiac troponin concentrations and their characteristics in larger cohort studies. Several of these studies showed that (slightly, even below the 99the percentile) elevated cardiac troponin concentrations reflect future or (sub)clinical disease, and are associated with an unfavorable prognosis, an increased risk for cardiovascular disease and mortality $77,78,83-87$. These findings indicate a potential role of cardiac troponins in secondary prevention. Though, there is no current consensus on the interpretation, treatment or follow-up of subjects with (slightly) elevated cardiac troponins without acute clinical symptoms. Future research in this field is required.

Lastly, the capability to measure cardiac troponin concentrations in a majority of subjects also facilitated the large-scale comparison of cTnT and cTnl concentrations. Although the current guidelines equally recommend both cardiac troponins for the diagnosis of acute myocardial infarction ${ }^{18}$, it became clear that not only the biochemical, but also the biological 
characteristics (for example the presence of a circadian rhythm and the variation in a healthy reference population) differ ${ }^{33,88-91}$. This leads to additional questions regarding dissimilarities between both cardiac troponins in diagnosis and prognosis.

\section{Outline of this thesis}

This thesis focuses on research that is related to the optimization of interpretation of cardiac troponin concentration for both diagnosis and prognosis.

Chapter 2 presents a case which illustrates four important challenges we face in the interpretation of cardiac troponins (discussed in the following chapters): First, the variation in cardiac troponin concentrations over the day (chapters 3 and 4). Second, the effect of impaired renal function on cardiac troponin levels (chapters 5 and 6). Third, the differences between cTnT and cTnl (chapters 7 and 8). Fourth, persistently elevated basal cardiac troponin concentrations and their clinical significance (chapters 9, 10 and 11).

Chapter 3 shows a diurnal rhythm that is present for cTnT, but not for cTnl, and examines its effect on the diagnosis of acute myocardial infarction.

Chapter 4 quantifies the hour-to-hour biological variation in cTnI and its time-dependency over 24 hours in subjects with or without chronic kidney disease.

Chapter 5 employs the circadian rhythm in CTnT concentrations, described in chapter 3, to examine if the kidneys, by impaired renal clearance, or the heart, by increased release, can be considered as the main driver behind elevated cTnT concentrations in chronic kidney disease patients.

Chapter 6 uses mass-spectrometry to study if cTnT can be identified in the urine of patients with acute myocardial injury.

Chapter 7 presents a series of experiments which examine the variation in measured concentrations after the addition of a standardized amount of cardiac troponins to the serum and plasma of different individuals.

Chapter 8 explores whether combining CTnT and cTnl might overcome limitations of individual cardiac troponins, and increase the number of rule-outs after a single blood draw. 
Chapters 9 and 10 describe the prevalence of basal elevated cardiac troponin concentrations in (frail) elderly, and assess the effects of prolonged exercise training on these levels.

Chapter 11 contains a meta-analysis to the prognostic value of cTnT and cTnl on cardiovascular and all-cause mortality in the general population.

The final chapter, chapter 12, contains a general discussion of the work presented in this thesis and provides directions for future research. 


\section{References}

1. Townsend N, Wilson L, Bhatnagar P, Wickramasinghe K, Rayner M, Nichols M. Cardiovascular disease in Europe: epidemiological update 2016. Eur Heart J 2016.

2. Mortality GBD, Causes of Death C. Global, regional, and national age-sex specific all-cause and cause-specific mortality for 240 causes of death, 1990-2013: a systematic analysis for the Global Burden of Disease Study 2013. Lancet 2015;385:117-71.

3. Volksgezondheidenzorg.info. 2016. at https://www.volksgezondheidenzorg.info.

4. OECD Better Life Index. 2016. at http://www.oecdbetterlifeindex.org/countries/netherlands/.

5. Roggeri DP, Roggeri A, Rossi E, et al. Direct healthcare costs and resource consumption after acute coronary syndrome: a real-life analysis of an Italian subpopulation. Eur J Prev Cardiol 2014;21:1090-6.

6. Roggeri A, Gnavi R, Dalmasso M, et al. Resource consumption and healthcare costs of acute coronary syndrome: a retrospective observational administrative database analysis. Crit Pathw Cardiol 2013;12:204-9.

7. Soekhlal RR, Burgers LT, Redekop WK, Tan SS. Treatment costs of acute myocardial infarction in the Netherlands. Neth Heart J 2013;21:230-5.

8. Nawar EW, Niska RW, Xu J. National Hospital Ambulatory Medical Care Survey: 2005 emergency department summary. Adv Data 2007:1-32.

9. Reichlin T, Hochholzer W, Bassetti S, et al. Early diagnosis of myocardial infarction with sensitive cardiac troponin assays. N Engl J Med 2009;361:858-67.

10. Reiter M, Twerenbold R, Reichlin T, et al. Early diagnosis of acute myocardial infarction in the elderly using more sensitive cardiac troponin assays. Eur Heart J 2011;32:1379-89.

11. Raskovalova T, Twerenbold R, Collinson PO, et al. Diagnostic accuracy of combined cardiac troponin and copeptin assessment for early rule-out of myocardial infarction: a systematic review and meta-analysis. Eur Heart J Acute Cardiovasc Care 2014;3:18-27.

12. Lipinski MJ, Baker NC, Escarcega RO, et al. Comparison of conventional and high-sensitivity troponin in patients with chest pain: a collaborative meta-analysis. Am Heart J 2015;169:6-16 e6.

13. Mockel M, Searle J, Hamm C, et al. Early discharge using single cardiac troponin and copeptin testing in patients with suspected acute coronary syndrome (ACS): a randomized, controlled clinical process study. Eur Heart J 2015;36:369-76.

14. Thygesen K, Mair J, Giannitsis E, et al. How to use high-sensitivity cardiac troponins in acute cardiac care. Eur Heart J 2012;33:2252-7.

15. Wenaweser P, Windecker S. Acute coronary syndromes: management and secondary prevention. Herz 2008;33:25-37.

16. Goodacre S, Thokala P, Carroll C, et al. Systematic review, meta-analysis and economic modelling of diagnostic strategies for suspected acute coronary syndrome. Health Technol Assess 2013;17:v-vi, 1-188.

17. Thygesen K, Alpert JS, Jaffe AS, et al. Third universal definition of myocardial infarction. Eur Heart J 2012;33:255167.

18. Roffi M, Patrono C, Collet JP, et al. 2015 ESC Guidelines for the management of acute coronary syndromes in patients presenting without persistent ST-segment elevation: Task Force for the Management of Acute Coronary Syndromes in Patients Presenting without Persistent ST-Segment Elevation of the European Society of Cardiology (ESC). Eur Heart J 2016;37:267-315.

19. Menown IB, Mackenzie G, Adgey AA. Optimizing the initial 12-lead electrocardiographic diagnosis of acute myocardial infarction. Eur Heart J 2000;21:275-83.

20. Freisinger E, Fuerstenberg T, Malyar NM, et al. German nationwide data on current trends and management of acute myocardial infarction: discrepancies between trials and real-life. Eur Heart J 2014;35:979-88.

21. McManus DD, Gore J, Yarzebski J, Spencer F, Lessard D, Goldberg RJ. Recent trends in the incidence, treatment, and outcomes of patients with STEMI and NSTEMI. Am J Med 2011;124:40-7.

22. Ruff CT, Braunwald E. The evolving epidemiology of acute coronary syndromes. Nat Rev Cardiol 2011;8:140-7. 
23. Danese E, Montagnana M. An historical approach to the diagnostic biomarkers of acute coronary syndrome. Ann Transl Med 2016;4:194.

24. Mythili S, Malathi N. Diagnostic markers of acute myocardial infarction. Biomed Rep 2015;3:743-8.

25. Wu AH. Cardiac markers: from enzymes to proteins, diagnosis to prognosis, laboratory to bedside. Ann Clin Lab Sci 1999;29:18-23.

26. Dolci A, Panteghini M. The exciting story of cardiac biomarkers: from retrospective detection to gold diagnostic standard for acute myocardial infarction and more. Clin Chim Acta 2006;369:179-87.

27. Vasan RS. Biomarkers of cardiovascular disease: molecular basis and practical considerations. Circulation 2006;113:2335-62.

28. Manolio T. Novel risk markers and clinical practice. N Engl J Med 2003;349:1587-9.

29. Eusebi P. Diagnostic accuracy measures. Cerebrovasc Dis 2013;36:267-72.

30. Linnet K, Bossuyt PM, Moons KG, Reitsma JB. Quantifying the accuracy of a diagnostic test or marker. Clin Chem 2012;58:1292-301.

31. Collinson PO, Gaze DC. Biomarkers of cardiovascular damage and dysfunction--an overview. Heart Lung Circ 2007;16 Suppl 3:S71-82.

32. de Lemos JA. Increasingly sensitive assays for cardiac troponins: a review. JAMA 2013;309:2262-9.

33. Katrukha IA. Human cardiac troponin complex. Structure and functions. Biochemistry (Mosc) 2013;78:1447-65. 34. Katus HA, Remppis A, Scheffold T, Diederich KW, Kuebler W. Intracellular compartmentation of cardiac troponin $T$ and its release kinetics in patients with reperfused and nonreperfused myocardial infarction. Am J Cardiol 1991;67:1360-7.

35. Remppis A, Scheffold T, Greten J, et al. Intracellular compartmentation of troponin T: release kinetics after global ischemia and calcium paradox in the isolated perfused rat heart. J Mol Cell Cardiol 1995;27:793-803.

36. Voss EM, Sharkey SW, Gernert AE, et al. Human and canine cardiac troponin $T$ and creatine kinase-MB distribution in normal and diseased myocardium. Infarct sizing using serum profiles. Arch Pathol Lab Med 1995;119:799-806.

37. Dhoot GK, Frearson N, Perry SV. Polymorphic forms of troponin T and troponin C and their localization in striated muscle cell types. Exp Cell Res 1979;122:339-50.

38. Mikawa T, Takeda S, Shimizu T, Kitaura T. Gene expression of myofibrillar proteins in single muscle fibers of adult chicken: micro two dimensional gel electrophoretic analysis. J Biochem 1981;89:1951-62.

39. Bucher EA, Maisonpierre PC, Konieczny SF, Emerson CP, Jr. Expression of the troponin complex genes: transcriptional coactivation during myoblast differentiation and independent control in heart and skeletal muscles. Mol Cell Biol 1988;8:4134-42.

40. Cummins B, Auckland ML, Cummins P. Cardiac-specific troponin-I radioimmunoassay in the diagnosis of acute myocardial infarction. Am Heart J 1987;113:1333-44.

41. Katus HA, Remppis A, Looser S, Hallermeier K, Scheffold T, Kubler W. Enzyme linked immuno assay of cardiac troponin $T$ for the detection of acute myocardial infarction in patients. J Mol Cell Cardiol 1989;21:1349-53.

42. Bodor GS, Porter S, Landt Y, Ladenson JH. Development of monoclonal antibodies for an assay of cardiac troponin-I and preliminary results in suspected cases of myocardial infarction. Clin Chem 1992;38:2203-14.

43. Adams JE, 3rd, Bodor GS, Davila-Roman VG, et al. Cardiac troponin I. A marker with high specificity for cardiac injury. Circulation 1993;88:101-6.

44. Hallermayer K, Klenner D, Vogel R. Use of recombinant human cardiac Troponin T for standardization of third generation Troponin T methods. Scand J Clin Lab Invest Suppl 1999;230:128-31.

45. Hermsen D, Apple F, Garcia-Beltran L, et al. Results from a multicenter evaluation of the 4th generation Elecsys Troponin T assay. Clin Lab 2007;53:1-9.

46. Sandoval Y, Smith SW, Apple FS. Present and Future of Cardiac Troponin in Clinical Practice: A Paradigm Shift to High-Sensitivity Assays. Am J Med 2016;129:354-65.

47. Giannitsis E, Kurz K, Hallermayer K, Jarausch J, Jaffe AS, Katus HA. Analytical validation of a high-sensitivity cardiac troponin T assay. Clin Chem 2010;56:254-61. 
48. Apple FS. A new season for cardiac troponin assays: it's time to keep a scorecard. Clin Chem 2009;55:1303-6. 49. Jarolim P. High sensitivity cardiac troponin assays in the clinical laboratories. Clin Chem Lab Med 2015;53:63552.

50. Cardinaels EP, Mingels AM, Jacobs LH, Meex SJ, Bekers O, van Dieijen-Visser MP. A comprehensive review of upper reference limits reported for (high-)sensitivity cardiac troponin assays: the challenges that lie ahead. Clin Chem Lab Med 2012;50:791-806.

51. Mueller C. Biomarkers and acute coronary syndromes: an update. Eur Heart J 2014;35:552-6.

52. Apple FS, Collinson PO, Biomarkers ITFoCAoC. Analytical characteristics of high-sensitivity cardiac troponin assays. Clin Chem 2012;58:54-61.

53. Mair J, Lindahl B, Muller C, et al. What to do when you question cardiac troponin values. Eur Heart J Acute Cardiovasc Care 2017:2048872617708973.

54. Thygesen K, Mair J, Katus H, et al. Recommendations for the use of cardiac troponin measurement in acute cardiac care. Eur Heart J 2010;31:2197-204.

55. Amsterdam EA, Wenger NK, Brindis RG, et al. 2014 AHA/ACC guideline for the management of patients with non-ST-elevation acute coronary syndromes: executive summary: a report of the American College of Cardiology/American Heart Association Task Force on Practice Guidelines. Circulation 2014;130:2354-94.

56. Armbruster DA, Pry T. Limit of blank, limit of detection and limit of quantitation. Clin Biochem Rev 2008;29 Suppl 1:S49-52.

57. Alpert JS, Antman E, Apple F, et al. Myocardial infarction redefined - A consensus document of the Joint European Society of Cardiology/American College of Cardiology Committee for the Redefinition of Myocardial Infarction. European Heart Journal 2000;21:1502-13.

58. Thygesen K, Alpert JS, White HD, et al. Universal definition of myocardial infarction. Circulation 2007;116:263453.

59. Apple FS, Jesse RL, Newby LK, et al. National Academy of Clinical Biochemistry and IFCC Committee for Standardization of Markers of Cardiac Damage Laboratory Medicine Practice Guidelines: analytical issues for biochemical markers of acute coronary syndromes. Clin Chem 2007;53:547-51.

60. Morrow DA, Cannon CP, Jesse RL, et al. National Academy of Clinical Biochemistry Laboratory Medicine Practice Guidelines: clinical characteristics and utilization of biochemical markers in acute coronary syndromes. Clin Chem 2007;53:552-74.

61. Keller T, Zeller T, Peetz D, et al. Sensitive troponin I assay in early diagnosis of acute myocardial infarction. N Engl J Med 2009;361:868-77.

62. Keller T, Zeller T, Ojeda F, et al. Serial changes in highly sensitive troponin I assay and early diagnosis of myocardial infarction. JAMA 2011;306:2684-93.

63. Giannitsis E, Becker M, Kurz K, Hess G, Zdunek D, Katus HA. High-sensitivity cardiac troponin T for early prediction of evolving non-ST-segment elevation myocardial infarction in patients with suspected acute coronary syndrome and negative troponin results on admission. Clin Chem 2010;56:642-50.

64. Haaf P, Drexler B, Reichlin T, et al. High-sensitivity cardiac troponin in the distinction of acute myocardial infarction from acute cardiac noncoronary artery disease. Circulation 2012;126:31-40.

65. Neumann JT, Sorensen NA, Schwemer T, et al. Diagnosis of Myocardial Infarction Using a High-Sensitivity Troponin I 1-Hour Algorithm. JAMA Cardiol 2016;1:397-404.

66. Mueller C, Giannitsis E, Christ M, et al. Multicenter Evaluation of a 0-Hour/1-Hour Algorithm in the Diagnosis of Myocardial Infarction With High-Sensitivity Cardiac Troponin T. Ann Emerg Med 2016;68:76-87 e4.

67. Jaeger C, Wildi K, Twerenbold R, et al. One-hour rule-in and rule-out of acute myocardial infarction using highsensitivity cardiac troponin I. Am Heart J 2016;171:92-102 e1-5.

68. Reichlin T, Schindler C, Drexler B, et al. One-hour rule-out and rule-in of acute myocardial infarction using highsensitivity cardiac troponin T. Arch Intern Med 2012;172:1211-8.

69. Reichlin T, Twerenbold R, Wildi K, et al. Prospective validation of a 1-hour algorithm to rule-out and rule-in acute myocardial infarction using a high-sensitivity cardiac troponin T assay. CMAJ 2015;187:E243-52. 
70. Rubini Gimenez M, Twerenbold R, Jaeger C, et al. One-hour rule-in and rule-out of acute myocardial infarction using high-sensitivity cardiac troponin I. Am J Med 2015;128:861-70 e4.

71. Newby LK, Jesse RL, Babb JD, et al. ACCF 2012 expert consensus document on practical clinical considerations in the interpretation of troponin elevations: a report of the American College of Cardiology Foundation task force on Clinical Expert Consensus Documents. J Am Coll Cardiol 2012;60:2427-63.

72. Peacock WFt, De Marco T, Fonarow GC, et al. Cardiac troponin and outcome in acute heart failure. N Engl J Med 2008;358:2117-26.

73. Muller-Bardorff M, Weidtmann B, Giannitsis E, Kurowski V, Katus HA. Release kinetics of cardiac troponin T in survivors of confirmed severe pulmonary embolism. Clin Chem 2002;48:673-5.

74. Sandhu R, Aronow WS, Rajdev A, et al. Relation of cardiac troponin I levels with in-hospital mortality in patients with ischemic stroke, intracerebral hemorrhage, and subarachnoid hemorrhage. Am J Cardiol 2008;102:632-4.

75. Masson S, Anand I, Favero C, et al. Serial measurement of cardiac troponin T using a highly sensitive assay in patients with chronic heart failure: data from 2 large randomized clinical trials. Circulation 2012;125:280-8.

76. Omland T, de Lemos JA, Sabatine MS, et al. A sensitive cardiac troponin T assay in stable coronary artery disease. N Engl J Med 2009;361:2538-47.

77. Everett BM, Cook NR, Magnone MC, et al. Sensitive cardiac troponin T assay and the risk of incident cardiovascular disease in women with and without diabetes mellitus: the Women's Health Study. Circulation 2011;123:2811-8.

78. deFilippi C, Seliger SL, Kelley W, et al. Interpreting cardiac troponin results from high-sensitivity assays in chronic kidney disease without acute coronary syndrome. Clin Chem 2012;58:1342-51.

79. Klinkenberg LJ, Luyten P, van der Linden N, et al. Cardiac Troponin T and I Release After a 30-km Run. Am J Cardiol 2016;118:281-7.

80. Mingels A, Jacobs L, Michielsen E, Swaanenburg J, Wodzig W, van Dieijen-Visser M. Reference population and marathon runner sera assessed by highly sensitive cardiac troponin $\mathrm{T}$ and commercial cardiac troponin $\mathrm{T}$ and I assays. Clin Chem 2009;55:101-8.

81. Twerenbold R, Wildi K, Jaeger C, et al. Optimal Cutoff Levels of More Sensitive Cardiac Troponin Assays for the Early Diagnosis of Myocardial Infarction in Patients With Renal Dysfunction. Circulation 2015;131:2041-50.

82. Chenevier-Gobeaux C, Meune C, Freund Y, et al. Influence of age and renal function on high-sensitivity cardiac troponin T diagnostic accuracy for the diagnosis of acute myocardial infarction. Am J Cardiol 2013;111:1701-7.

83. Blankenberg S, Salomaa V, Makarova N, et al. Troponin I and cardiovascular risk prediction in the general population: the BiomarCaRE consortium. Eur Heart J 2016;37:2428-37.

84. Sze J, Mooney J, Barzi F, Hillis GS, Chow CK. Cardiac Troponin and its Relationship to Cardiovascular Outcomes in Community Populations - A Systematic Review and Meta-analysis. Heart Lung Circ 2016;25:217-28.

85. Haaf $P$, Reichlin $T$, Twerenbold R, et al. Risk stratification in patients with acute chest pain using three highsensitivity cardiac troponin assays. Eur Heart J 2014;35:365-75.

86. van Wijk S, Jacobs L, Eurlings LW, et al. Troponin T measurements by high-sensitivity vs conventional assays for risk stratification in acute dyspnea. Clin Chem 2012;58:284-92.

87. Saunders JT, Nambi V, de Lemos JA, et al. Cardiac troponin T measured by a highly sensitive assay predicts coronary heart disease, heart failure, and mortality in the Atherosclerosis Risk in Communities Study. Circulation 2011;123:1367-76.

88. Klinkenberg LJ, van Dijk JW, Tan FE, van Loon LJ, van Dieijen-Visser MP, Meex SJ. Circulating cardiac troponin T exhibits a diurnal rhythm. J Am Coll Cardiol 2014;63:1788-95.

89. Kimenai DM, Henry RM, van der Kallen CJ, et al. Direct comparison of clinical decision limits for cardiac troponin T and I. Heart 2016;102:610-6.

90. Wildi K, Gimenez MR, Twerenbold R, et al. Misdiagnosis of Myocardial Infarction Related to Limitations of the Current Regulatory Approach to Define Clinical Decision Values for Cardiac Troponin. Circulation 2015;131:2032-40. 91. Rubini Gimenez M, Twerenbold R, Reichlin T, et al. Direct comparison of high-sensitivity-cardiac troponin I vs. T for the early diagnosis of acute myocardial infarction. Eur Heart J 2014;35:2303-11. 


\section{Strong diurnal rhythm of troponin T, but}

not troponin I, in a patient with renal

\section{dysfunction}

Noreen van der Linden, Tom Cornelis, Lieke Klinkenberg, Dorien Kimenai, Judith Hilderink, Elisabeth Litjens, Jeroen Kooman, Otto Bekers, Marja van Dieijen-Visser, Steven Meex - Int J Cardiol: 2016 oct 15;221:287-8. 


\section{Introduction}

Cardiac troponin T (cTnT) and I (cTnI), measured with high-sensitive assays, are the preferred biomarkers for the diagnosis of acute myocardial infarction ${ }^{1,2}$. Serial cardiac troponin measurements, and the interpretation of a 'troponin rise-or-fall' in patients with chest pain discriminate between acute and chronic troponin elevations ${ }^{1}$. This diagnostic concept assumes that troponin levels in patients without acute cardiac injury fluctuate randomly around an individual's homeostatic setpoint. However, it has recently been demonstrated that $\mathrm{cTnT}$ exhibits a diurnal rhythm ${ }^{3,4}$. The amplitude of the diurnal cTnT rhythm and its diagnostic implication in patients with chronically elevated cTnT levels are unexplored. Also, whether cTnT and cTnl are equally affected by rhythmic diurnal oscillation is unknown.

\section{Methods}

A 63-year-old woman with decreased renal function (estimated Glomerular Filtration Rate (eGFR) $14 \mathrm{ml} / \mathrm{min} / 1.73 \mathrm{~m}^{2}$ ) was included in our observational study to the effect of renal function on the diurnal rhythm of cTnT and cTnl (ClinicalTrials.gov: NCT02210897). This study was carried out according to the principles of the Declaration of Helsinki and approved by the Institutional Review Board and Ethics Committee of Maastricht University Medical Center. The participant provided written informed consent. Her medical history included nephroangiosclerosis, dyslipidemia, hypertension, pacemaker implantation for chronotropic incompetence, hyperparathyroidism caused by parathyroid adenoma, aortic insufficiency, and gout. Chronically elevated cardiac troponin levels in this patient were first observed in 2011, when she was evaluated at the emergency department after a collapse. At the time, laboratory analysis with a conventional, non-high-sensitive, assay showed an increased cTnT level $\left(0.84 \mu \mathrm{g} / \mathrm{L} ; 99^{\text {th }}\right.$ percentile $\left.<0.01 \mu \mathrm{g} / \mathrm{L}\right)$, which remained elevated during hospitalization $(0.77 \mu \mathrm{g} / \mathrm{L}$ and $0.79 \mu \mathrm{g} / \mathrm{L}, 6$ and 16 hours after presentation respectively). Coronary angiography and pulmonary CT angiography found no substrate, and the elevated cardiac troponin levels were therefore considered unrelated to an acute coronary event or pulmonary embolism. In the end, she was discharged with a diagnosis of vasovagal collapse. As part of the present study, she was restricted to the laboratory environment over a period 
of 25 hours and serial venous blood samples were drawn every hour. She took her regular medication (allopurinol, amlodipine, doxazosine, ferrofumarate, furosemide, irbesartan, sodium bicarbonate, nebivolol, pantoprazole, prednisolone and sevelamer) and was completely asymptomatic during these 25 hours. High-sensitivity cardiac troponin T (hscTnT, Roche Cobas) and I (hs-cTnl, Abbott Architect) were measured in duplicate in all samples. Analyses were performed using IBM SPSS Statistics for Windows 23.0 (IBM Corp., Armonk, New York, USA) and GraphPad Prism version 5.03 for Windows (GraphPad Software, San Diego, California, USA).

\section{Results}

In line with laboratory results from 2011, baseline hs-cTnT concentration at the start of the study, day $18.30 \mathrm{AM}$, was highly elevated and strongly exceeded the $99^{\text {th }}$ percentile threshold $\left(147.7 \mathrm{ng} / \mathrm{L}, 99^{\text {th }}\right.$ percentile $\left.=14 \mathrm{ng} / \mathrm{L}\right)$. Interestingly, hs-cTnl at baseline was within the reference range $\left(10.3 \mathrm{ng} / \mathrm{L}, 99^{\text {th }}\right.$ percentile $\left.=26.2 \mathrm{ng} / \mathrm{L}\right)$. hs-cTnT concentrations showed a diurnal rhythm and fitted a cosine model ${ }^{5}\left(R^{2}=0.90\right)$, with a maximal concentration change over 24 hours of $50.9 \mathrm{ng} / \mathrm{L}$. In contrast, hs-cTnl levels remained virtually unchanged over time with a maximal difference of only $2.6 \mathrm{ng} / \mathrm{L}$ over 24 hours and no visual cosine fit. The concentrations of hs-cTnT and hs-cTnl over time are shown in figure 1. hs-cTnT levels exceeded the diagnostic criteria, both baseline level and 1-hour delta-change values, of the current European Society of Cardiology (ESC) guidelines to rule-in subjects for $\mathrm{AMI}^{1}$.

\section{Conclusion}

This observation warrants further evaluation of the diurnal troponin T rhythm in subjects with chronically elevated hs-cTnT levels, and examination of its effect on the diagnostic accuracy in patients with chronically elevated troponin levels. 


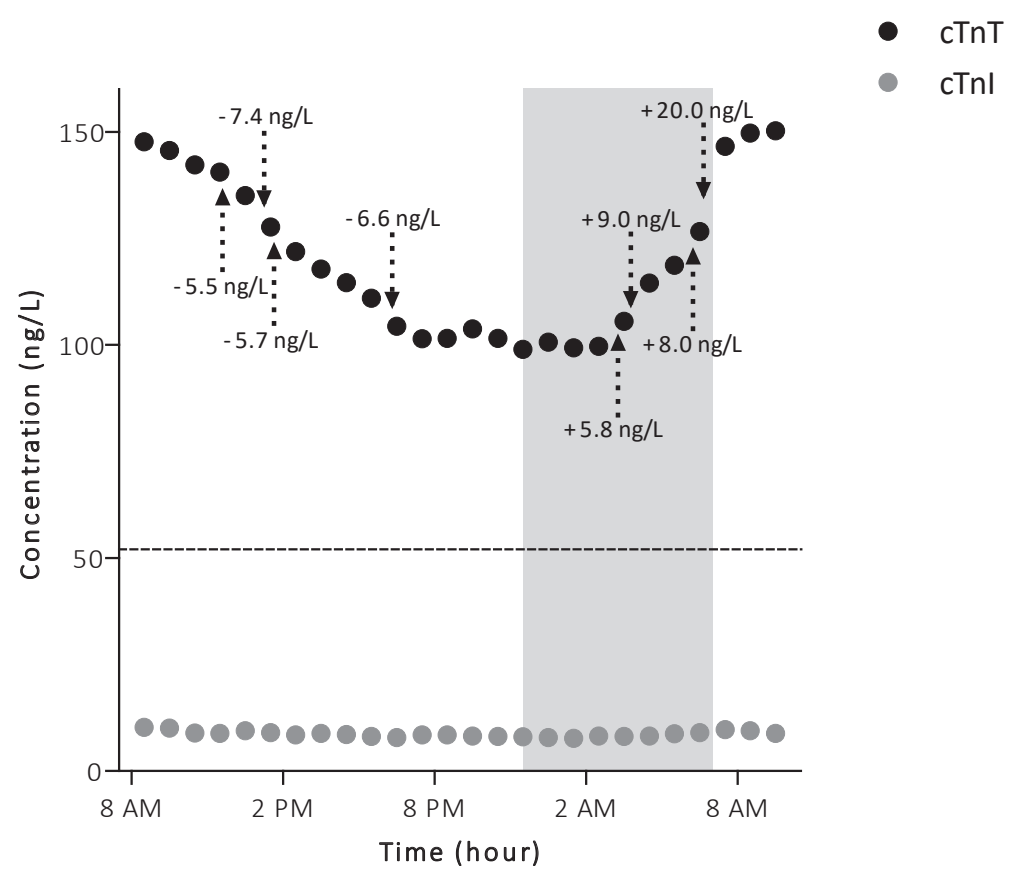

Figure 1. The course of hs-cTnT and hs-cTnl in a 63-year old woman with decreased renal function. The dotted line indicates the cut-off level for the immediate ruling-in of suspected non-ST-elevation myocardial infarction without additional serial testing using high-sensitive cardiac troponin assays at presentation as recently published in the $\mathrm{Oh} / \mathrm{h}$ rule-in and rule-out algorithms of the ESC guidelines. According to this algorithm patients are ruled in the acute coronary syndrome treatment protocol if they present with a concentration of hs-cTnT (Roche) or hs-cTnl (Abbott) above $52 \mathrm{ng} / \mathrm{L}$ (immediate rule-in without additional serial testing) or when hs-cTnT (Roche) or hs-cTnl (Abbott) concentration increases in the first hour with 5 or $6 \mathrm{ng} / \mathrm{l}$, respectively ${ }^{1,6,7}$. The 1 h delta values for hs-cTnT in this patient vary between 0.1 and $20.0 \mathrm{ng} / \mathrm{L}$, and exceed the cut-off value of $5 \mathrm{ng} / \mathrm{L}$ eight times, especially around noon and in the early morning. The $3 \mathrm{~h}$ delta values for hs-cTnT vary between $0.1 \mathrm{ng} / \mathrm{L}$ and $32.1 \mathrm{ng} / \mathrm{L}$. The subject slept in supine position between 11.30 PM and 7.00 AM (shaded area). 


\section{References}

1. Roffi M, Patrono C, Collet JP, et al. 2015 ESC Guidelines for the management of acute coronary syndromes in patients presenting without persistent ST-segment elevation: Task Force for the Management of Acute Coronary Syndromes in Patients Presenting without Persistent ST-Segment Elevation of the European Society of Cardiology (ESC). Eur Heart J 2016;37:267-315.

2. Mills NL, Churchhouse AM, Lee KK, et al. Implementation of a sensitive troponin I assay and risk of recurrent myocardial infarction and death in patients with suspected acute coronary syndrome. JAMA 2011;305:1210-6.

3. Klinkenberg $L$, van Dijk JW, Tan FE, van Loon $\sqcup$, van Dieijen-Visser MP, Meex SJ. Circulating cardiac troponin T exhibits a diurnal rhythm. J Am Coll Cardiol 2014;63:1788-95.

4. Aakre KM, Roraas T, Petersen PH, et al. Weekly and 90-minute biological variations in cardiac troponin $\mathrm{T}$ and cardiac troponin I in hemodialysis patients and healthy controls. Clin Chem 2014;60:838-47.

5. Nelson W, Tong YL, Lee JK, Halberg F. Methods for cosinor-rhythmometry. Chronobiologia 1979;6:305-23. 6. Reichlin T, Schindler C, Drexler B, et al. One-hour rule-out and rule-in of acute myocardial infarction using highsensitivity cardiac troponin T. Arch Intern Med 2012;172:1211-8.

7. Rubini Gimenez M, Twerenbold R, Jaeger C, et al. One-hour rule-in and rule-out of acute myocardial infarction using high-sensitivity cardiac troponin I. Am J Med 2015;128:861-70 e4. 
Cardiac Troponins: State of the (He)Art 


\section{Diurnal rhythm of cardiac troponin:}

\section{consequences for the diagnosis of acute myocardial infarction}

Lieke Klinkenberg*, Karin Wildi*, Noreen van der Linden, Imre Kouw, Marijke Niens, Raphael Twerenbold, Maria Rubini Gimenez, Christian Puelacher, Daniel Neuhaus, Petra Hillinger, Thomas Nestelberger, Jasper Boeddinghaus, Karin Grimm, Zaid Sabti, Judith Bons, Jeroen van Suijlen, Frans Tan, Joop ten Kate, Otto Bekers, Luc van Loon, Marja van Diejen-Visser, Christian Mueller, Steven Meex - Clin Chem, 2016 Dec;62(12):1602-1611 * equal contribution 


\section{Abstract}

\section{Background}

Interpretation of serial high-sensitivity troponin (hs-cTn) measurements for the diagnosis of acute myocardial infarction (AMI) assumes random fluctuation of hs-cTn around an individual's homeostatic set-point. The aim of this study was to challenge this diagnostic concept.

\section{Methods}

Study-1 examined the presence of a diurnal hs-cTn rhythm by hourly blood sampling, day-and-night, in 24 individuals without a recent history of AMI. Study-2 assessed morning versus evening diagnostic accuracy of hs-cTnT and hs-cTnl in a prospective multicenter diagnostic study of 2782 unselected patients, presenting to the emergency department with acute chest pain.

\section{Results}

In study-1, hs-cTnT, but not hs-cTnl, exhibited a diurnal rhythm, characterized by gradually decreasing concentrations throughout daytime, rising concentrations during nighttime, to peak concentrations in the morning (mean 16.2ng/L at 8:30A.M and 12.1ng/L at 7:30P.M.). In study-2, the hs-cTnT rhythm was confirmed by higher hs-cTnT levels in early-morning presenters compared to evening presenters with an adjudicated diagnosis of non-cardiac disease. The diagnostic accuracy (area under the receiveroperator-characteristic curve (AUC)) of hs-cTnT at presentation, 1-hour, and for the combination of absolute changes with presenting concentration, were very high and comparable among patients presenting early-morning as compared to evening (all AUC>0.93). Hs-cTnl exhibited no diurnal rhythm with no differences in AUC among early-morning and evening presenters.

\section{Conclusions}

Rhythmic diurnal variation of hs-cTnT is a general phenomenon that is not seen with hs-cTnl. While the diurnal hs-cTnT rhythm does not seem to affect the diagnostic accuracy of hs-cTnT for AMI, it should be considered when using hs-cTnT for screening purposes. 


\section{Introduction}

The early diagnosis of acute myocardial infarction (AMI) is mandatory for the timely initiation of evidence-based therapy and based on three key diagnostic tools: clinical assessment, the ECG, and cardiac troponin (cTn), a quantitative marker of cardiomyocyte injury ${ }^{1}$. Troponin assays target one of two proteins - cardiac troponin T (cTnT) or cardiac troponin I (cTnl) with distinct biochemical properties, but comparable diagnostic performance ${ }^{2}$. The advent of high-sensitivity cTn assays for both cTnT (hs-cTnT) and cTnl (hs-cTnl) has expedited the early diagnosis of $\mathrm{AMI}^{3-5}$, but also resulted in the detection of previously unnoticed cardiomyocyte injury in multiple other conditions such as diabetes, chronic kidney disease, frailty, or even apparently healthy elderly individuals ${ }^{6,7}$. Key to accurately discriminate between acute and chronic causes of cardiomyocyte injury is serial testing and the assessment of kinetic hs-cTn changes as recommended in the current guidelines ${ }^{8-11}$. A critical assumption when interpreting changes between serial measurements is that hs-cTn blood concentrations fluctuate randomly around a homeostatic set-point. This dogma of random fluctuation was recently challenged by the intriguing observation that hs-cTnT exhibited a diurnal rhythm in a small group of male subjects with type 2 diabetes ${ }^{12}$. The diurnal hs-cTnT rhythm was characterized by peak-levels during morning hours, a gradual decrease during the day, and a rising pattern at nighttime ${ }^{12}$.

The generalizability of the diurnal rhythm of hs-cTnT beyond male type 2 diabetes patients and whether hs-cTnT and hs-cTnl are equally affected is unknown. It is also unknown, whether -if confirmed- diurnal rhythmic oscillation in hs-cTn blood concentration affect the diagnostic accuracy of hs-cTn for AMI. This possibility is substantiated by the observation that the magnitude of physiological change over the day, exceeded clinically applied delta-change criteria for $\mathrm{AMI}$ in some individuals ${ }^{1,12}$.

The present study was conducted to quantify the rhythmic diurnal oscillation of hs-cTnT and hs-cTnl in currently asymptomatic women and men. Next, we analyzed data from a multicenter diagnostic study, enrolling patients presenting to the emergency department with suspected $A M I$, to determine whether rhythmic diurnal oscillation and thereby the time of presentation affects the diagnostic accuracy of hs-cTnT and hs-cTnl. 


\section{Materials and methods}

Two studies were conducted: Study 1 was a standardized biological variation study with hourly blood sampling day and night, to ascertain and quantify circadian rhythms of hs-cTnT and hs-cTnl (January 2013 to May 2014); Study 2 was a prospective diagnostic study (APACE; Advantageous $\underline{\text { Predictors of }}$ Acute Coronary syndrome Evaluation). APACE is an ongoing prospective international multicenter study aiming to advance the early diagnosis of $\mathrm{AMI}^{2,4,13,14}$. Both studies were carried out according to the principles of the Declaration of Helsinki and approved by the local Institutional Review Board and Ethics Committee at each institution. Written informed consent was obtained from all participants.

\section{Study 1: Diurnal rhythm of cardiac troponin under standardized conditions}

The study population consisted of 24 individuals; 19 males and 5 females (mean age $72 \pm 7$ years, Supplementary Table 1). Exclusion criteria were: a history of AMI or stroke less than 12 months prior to inclusion. The diurnal hs-cTnT variation of 7 male subjects has been described before as part of a biological variation study of hs-cTnT in patients with type 2 diabetes (Supplementary Figure $1 \mathrm{~A}$ and 2, participants $4,5,7,11,16,20,24)^{12}$. To extend these pilot findings to both sexes and individuals with normal glucose homeostasis, 17 additional subjects (12 men, 5 women) were included in the present study. Participants were asked to refrain from exhaustive physical activities and exercise training 2 days before the test period. After an overnight fast, subjects arrived at the laboratory by car or public transportation. From 8.30 AM till 9.30 AM the next day, subjects were restricted to the laboratory environment and sampled hourly $(8 \mathrm{~mL})$ via an antecubital venous catheter. At night (11.30 PM till 7.00 AM), extension lines were used to minimize disturbance of participants' sleep. Meals were consumed at 8.30 AM, 12.30 PM and 5.30 PM (breakfast, lunch and dinner, respectively). Blood samples were collected in both serum (sensitive cTnl [s-cTnl] Beckman Coulter) and EDTA tubes (hs-cTnT Roche and hs-cTnl Abbott) and measured in duplicate. 


\section{Study 2: Diurnal rhythm of cardiac troponin and diagnostic accuracy}

To investigate the clinical effects of rhythmic diurnal hs-cTn oscillations, we evaluated whether the time of presentation to the emergency department affected the diagnostic accuracy of hs-cTn for AMI in the APACE study. The study population consisted of 2782 consecutive patients older than 18 years presenting to the emergency department between April 2006 and June 2013 with symptoms suggestive of AMI with onset or peak of symptoms within the last 12 hours (Supplementary Table 2). Patients with renal failure requiring dialysis were excluded from the study.

For this analysis patients were excluded if the adjudicated diagnosis was ST-elevation MI $(n=108)$ (as biomarkers in general do not have a role in the rapid diagnosis of ST-elevation $\mathrm{AMI}$ ), if the final diagnosis remained unclear after adjudication and at least one cTn level was elevated (possibly indicating presence of AMI) ( $n=92$ ), or if the exact presentation time was not recorded $(n=48)$.

Adjudication of the final diagnosis and follow-up of the patients is extensively described in the Supplementary Methods. Briefly, two independent cardiologists adjudicated the final diagnosis by reviewing all available medical records - patient history, physical examination, results of laboratory testing (including cTn levels), radiologic testing, ECG, echocardiography, cardiac exercise test, lesion severity and morphology in coronary angiography - pertaining to the patient from the time of ED presentation to 90-day follow up. In situations of disagreement about the diagnosis, cases were reviewed and adjudicated in conjunction with a third cardiologist.

Cosinor rhythmometry results from study 1 were used to divide the day in two timeframes: a 'hs-cTn high level phase' (early-morning) and a 'hs-cTn low level phase' (evening). Diagnostic accuracy of hs-cTn at presentation, at $1 \mathrm{~h}$, and the combination of absolute changes with baseline concentration were the primary diagnostic end points, as they reflect the most important diagnostic variables in clinical practice ${ }^{1,9,10}$. 


\section{Laboratory analysis}

Details on the four cTn assays (hs-cTnT Roche Diagnostics, hs-cTnl Abbott Diagnostics, hscTnl Siemens, s-cTnl Beckman Coulter) used in this manuscript are described in the Supplementary Methods.

\section{Statistical analysis}

Diurnal troponin changes were analyzed by fitting the data to a cosine curve by using the method of cosinor-rhythmometry ${ }^{12,15}$. Evidence of a diurnal rhythm was indicated by a significant cosinor model fit. Receiver-operating characteristics (ROC) curves were constructed to assess the sensitivity and specificity of hs-cTn for the diagnosis of AMI in earlymorning presenters and evening presenters. The comparison of independent areas under the ROC curves (AUC) was performed as recommend by Hanley and McNeil ${ }^{16}$. Similarly, AUCs were compared in early-morning presenters and evening presenters for the prognostic accuracy of hs-cTn at presentation to predict all-cause mortality during follow-up.

All hypothesis testing was two-tailed and a p-value of less than 0.05 was considered statistically significant. Analyses were performed using IBM SPSS Statistics for Windows 22.0 (SPSS Inc, Chicago, IL) and MedCalc 10.3.4 (MedCalc Software, Mariakerke, Belgium).

\section{Results}

\section{High-sensitivity cardiac troponin T exhibits a diurnal rhythm}

To investigate the diurnal rhythm of hs-cTn, blood samples were collected hourly over a time span of 25h (8.30 AM to 9.30 AM the next day - Supplementary Figure 1). In all participants, we found rhythmic circadian hs-cTnT variation, characterized by gradually decreasing concentrations throughout daytime (mean $16.2 \mathrm{ng} / \mathrm{L}$ at $8.30 \mathrm{AM}$ and mean $12.1 \mathrm{ng} / \mathrm{L}$ at 7.30 PM) and rising concentrations during nighttime, to peak concentrations in the morning (mean $16.2 \mathrm{ng} / \mathrm{L}$ at 8:30A.M. the next day - Figure 1A, Supplementary Figure 2-4). Subjects with the highest hs-cTnT concentrations displayed the largest amplitude of the diurnal oscillation, illustrated by the highly significant correlation between hs-cTnT level at 8.30 AM and the range of individual changes during the $25 \mathrm{~h}$ period (Pearson's $R^{2} 0.70 ; p<0.001-$ 
Supplementary Figure 5). Based on the rhythmic variation, we tested the hs-cTnT data for a periodic signal using single cosinor analysis under the assumption of a $24 \mathrm{~h}$ period. In all but one individual (participant 1), the observed pattern correlated significantly to the fitted cosine model, as described by the mesor, amplitude and acrophase (range $R^{2}$ 0.44-0.91; all $p \leq 0.001$ - Table 1). Correction for possible posture-induced changes in plasma volume did not abrogate the diurnal rhythm (Supplementary Figure 6) ${ }^{17}$. In addition, we could not relate the diurnal rhythm to the $25 \mathrm{~h}$ variation in cortisol, thyroid stimulating hormone or testosterone in terms of the time of peak and the maximal individual changes during the day (Supplementary Methods, Supplementary Figure 7).

A

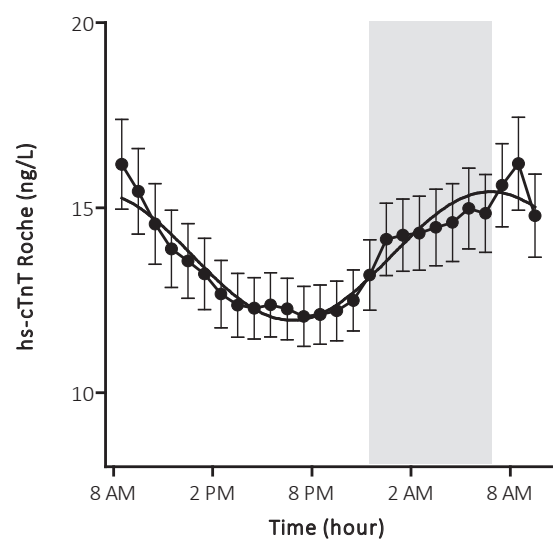

B

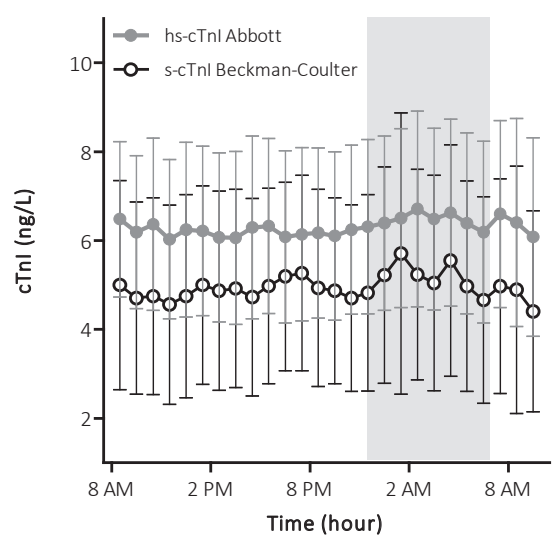

Figure 1. Diurnal variation of hs-cTnT, but not hs-cTnl, is a general phenomenon. A) Mean \pm SEM 25h hs-cTnT profile and fitted cosine curve. Subjects slept in a supine position between 11.30 PM and 7.00 AM (shaded area). The curve is described as $Z(t)=13.70+1.7 \cdot \cos (15 t-337.19)$, with a $R 2$ of $0.92(p<0.001)$. The time of peak was calculated at 6.58 AM. B) Mean \pm SEM $25 \mathrm{~h} \mathrm{cTnl} \mathrm{profile.}$

\section{High-sensitivity troponin I levels fluctuate at random}

Distinct from hs-cTnT, hs-cTnl (Abbott) and s-cTnl (Beckman Coulter) levels fluctuated randomly around the subject's homeostatic setpoint. Mean hs-cTnl and s-cTnl concentrations were $6.5 \mathrm{ng} / \mathrm{L}$ and $5.0 \mathrm{ng} / \mathrm{L}$ at $8.30 \mathrm{AM}$ and $6.1 \mathrm{ng} / \mathrm{L}$ and $5.3 \mathrm{ng} / \mathrm{L}$ at $7.30 \mathrm{PM}$, respectively (Figure 1B, Supplementary Figure 8 and 9). Accordingly, in all but one participant, the $25 \mathrm{~h}$ hs-cTnl and s-cTnl profile did not fit significantly to a cosine curve. Even 
participant 24, with hs-cTnl and s-cTnl values above the $99^{\text {th }}$ percentile upper reference limit, and with the most prominent diurnal oscillations for hs-cTnT, showed relatively stable hscTnl and s-cTnl levels during the 25h test period (Supplementary Figure 8 and 9). One individual had a hs-cTnl day-curve that could be fitted to a cosinus model (Supplementary Figure 8, participant 7). This curve, however, was characterized by an acrophase that was almost $10 \mathrm{~h}$ out of phase compared to the mean hs-cTnT acrophase (10.30 PM vs. mean hscTnT acrophase of 6.58 AM).

\section{Diurnal rhythm of high-sensitivity cardiac troponin in chest pain patients}

To investigate whether rhythmic diurnal hs-cTn oscillation is also evident in a clinical setting, we compared hs-cTnT (Roche) and hs-cTnl (Abbott and Siemens) concentrations from 2782 patients included in the APACE trial. Baseline hs-cTn values were available for 2700, 2281 and 1812 patients (Roche, Abbott and Siemens, respectively). The adjudicated final diagnosis was AMI in $17.5 \%$, unstable angina in $9.8 \%$, cardiac non-coronary disease in $14.3 \%$, noncardiac chest pain in 53.3\%, and unknown (but not AMI) in 5.1\%. In a multivariate analysis, admission time was a marginally significant predictor of baseline hs-cTnT values (Supplementary Table 3), but not hs-cTnl Abbott and hs-cTnl Siemens (data not shown).

APACE patients were classified in one of two timeframes that were derived from the observed diurnal rhythm of hs-cTnT under standardized conditions (Figure 1A): a hs-cTnT high-level phase in the early-morning (11.00 PM till 2.00 PM) and a hs-cTnT low-level phase in the evening (2.00 PM till 11.00 PM). Hs-cTnT also showed a diurnal rhythm in patients with adjudicated diagnoses of non-cardiac disease and other cardiac disorders with significantly higher hs-cTnT levels in early-morning presenters compared to evening presenters. These differences were statistically significant, but small in absolute terms, and were not seen in patients with AMI or unstable angina (Table 2, Supplementary Table 4).

The diurnal rhythm of hs-cTnT overall did not affect diagnostic accuracy for AMI. Diagnostic accuracy of hs-cTnT as quantified by the area under the receiver-operating characteristics curve (AUC) at presentation, at $1 \mathrm{~h}$ and with the combination of absolute changes with baseline concentration were very high and comparable among patients presenting in the 
Table 1. The individual diurnal variation of hs-cTnT is significantly described by a cosinor model

\begin{tabular}{|c|c|c|c|c|c|}
\hline Participant ${ }^{\mathrm{a}}$ & Mesor (ng/L) & Amplitude (ng/L) & Acrophase (h) & $\mathrm{R}^{2}$ & P-value \\
\hline 1 & 5.6 & 0.2 & $10.45 \mathrm{AM}$ & 0.23 & 0.05 \\
\hline 2 & 7.3 & 1.2 & $5.39 \mathrm{AM}$ & 0.82 & $<0.001^{*}$ \\
\hline 3 & 9.4 & 2.4 & $5.47 \mathrm{AM}$ & 0.89 & $<0.001^{*}$ \\
\hline 4 & 8.4 & 1.7 & 7.16 AM & 0.66 & $<0.001^{*}$ \\
\hline 5 & 8.6 & 0.9 & $6.29 \mathrm{AM}$ & 0.65 & $<0.001^{*}$ \\
\hline 6 & 8.9 & 0.9 & $5.01 \mathrm{AM}$ & 0.70 & $<0.001^{*}$ \\
\hline 7 & 10.0 & 1.0 & $7.35 \mathrm{AM}$ & 0.55 & $<0.001^{*}$ \\
\hline 8 & 10.6 & 0.9 & 5.57 AM & 0.54 & $<0.001^{*}$ \\
\hline 9 & 10.5 & 0.9 & $5.12 \mathrm{AM}$ & 0.68 & $<0.001^{*}$ \\
\hline 10 & 12.7 & 0.7 & $2.56 \mathrm{AM}$ & 0.44 & $0.001^{*}$ \\
\hline 11 & 12.9 & 2.4 & $6.03 \mathrm{AM}$ & 0.81 & $<0.001^{*}$ \\
\hline 12 & 12.8 & 1.3 & $5.18 \mathrm{AM}$ & 0.64 & $<0.001^{*}$ \\
\hline 13 & 15.6 & 3.8 & $6.03 \mathrm{AM}$ & 0.81 & $<0.001^{*}$ \\
\hline 14 & 13.8 & 2.2 & $8.17 \mathrm{AM}$ & 0.86 & $<0.001^{*}$ \\
\hline 15 & 15.3 & 2.7 & $8.53 \mathrm{AM}$ & 0.77 & $<0.001^{*}$ \\
\hline 16 & 17.5 & 4.0 & $6.37 \mathrm{AM}$ & 0.91 & $<0.001^{*}$ \\
\hline 17 & 16.3 & 1.9 & 10.26 AM & 0.57 & $<0.001^{*}$ \\
\hline 18 & 16.1 & 1.4 & $8.26 \mathrm{AM}$ & 0.79 & $<0.001^{*}$ \\
\hline 19 & 19.7 & 3.7 & $5.29 \mathrm{AM}$ & 0.90 & $<0.001^{*}$ \\
\hline 20 & 19.5 & 4.1 & $9.08 \mathrm{AM}$ & 0.80 & $<0.001^{*}$ \\
\hline 21 & 19.4 & 2.2 & $4.35 \mathrm{AM}$ & 0.86 & $<0.001^{*}$ \\
\hline 22 & 17.8 & 1.9 & $5.29 \mathrm{AM}$ & 0.79 & $<0.001^{*}$ \\
\hline 23 & 18.3 & 2.4 & $8.19 \mathrm{AM}$ & 0.82 & $<0.001^{*}$ \\
\hline 24 & 21.7 & 2.3 & 9.18 AM & 0.74 & $<0.001^{*}$ \\
\hline
\end{tabular}

The cosinor model is described as: $Z(t)=M+A \cdot \cos (\omega t+\phi)+e(t)$, where $Z(t)$ represents the measured hs-cTnT concentration at a given time ( $\mathrm{t}$ ), $\mathrm{M}$ the mesor (value about which oscillation occurs), $\mathrm{A}$ the amplitude (half the difference between the peak and the nadir value), $\omega$ the angular frequency (degrees per unit time, with $360^{\circ}$ representing a complete cycle), $\phi$ the acrophase (timing of maximal value in degrees) and e(t) the error between the cosinor model and the measurement. aSubjects were ranked on the basis of their visual range of hs-cTnT concentration over the day: generally, high subject numbers correspond to a wider hs-cTnT dispersion. The numbering used in Table 1 is extended to Supplementary Figure 1, 2, 8, 9 allowing direct comparisons throughout the paper.

*Significant after Bonferroni correction for multiple testing, $p=0.0021(0.05 / 24$ 
early-morning and patients presenting in the evening (all AUC >0.90; Table 3; Figure 2). In contrast, AUC of absolute changes for hs-cTnT within the first hour were significantly lower in the early-morning ( $A \cup C$ C.90, 95\% Cl 0.89-0.92) as compared to the evening (AUC 0.95, $95 \% \mathrm{Cl}$ 0.93-0.97, $\mathrm{p}=0.01$ ). No significant difference was seen in absolute changes within $2 \mathrm{~h}$, $3 \mathrm{~h}$ and $6 \mathrm{~h}$ between the two timeframes (Supplementary Table 5a). An additional analysis comparing diagnostic accuracy of absolute changes of day presenters (8 AM to 8 PM) and night presenters (8 PM to 8 AM) showed no systematic difference (Supplementary Table 5b). In patients presenting very early ( $\leq 3$ hours) after symptom onset ( $n=1490,16.3 \%$ with AMI), differences in AUC between early-morning presenters and patients presenting in the evening seemed to be more pronounced but without systematic significance (Table 4).

The prognostic accuracy of hs-cTnT at presentation for all-cause mortality within one year, as quantified by the AUC was moderate-to-high and comparable among all adjudicated diagnoses between early morning presenters and evening presenters (Supplementary Table $6)$.

Hs-cTnl (Abbott and Siemens) did not exhibit a diurnal rhythm, levels were similar among all adjudicated diagnoses between early-morning presenters and evening presenters (Table 2, Supplementary Table 4). In addition, diagnostic accuracy of hs-cTnl was very high and comparable among patients presenting in the early-morning and patients presenting in the evening (all AUC > 0.92, Table 3, Table 4, Supplementary Table 5). 
Table 2. Early-morning and evening levels of hs-cTnT and hs-cTnl according to final diagnosis

\begin{tabular}{|c|c|c|c|c|}
\hline & & $\begin{array}{l}\text { Early-morning } \\
11.00 \mathrm{PM}-2.00 \mathrm{PM}\end{array}$ & $\begin{array}{l}\text { Evening } \\
2.00 \mathrm{PM}-11.00 \mathrm{PM}\end{array}$ & \\
\hline Timepoint & Adjudicated diagnosis & Median (IQR) & Median (IQR) & p-value \\
\hline \multicolumn{5}{|c|}{ hs-cTnT Roche, $n g / L$} \\
\hline \multirow[t]{5}{*}{ Oh } & AMI & $60(28-153)$ & $60(25-119)$ & 0.94 \\
\hline & Unstable angina & $11(7-16)$ & $12(6-16)$ & 0.73 \\
\hline & Cardiac cause not $C A D^{b}$ & $14(7-30)$ & $10(6-26)$ & 0.04 \\
\hline & Non-cardiac cause & $6(4-10)$ & $5(3-8)$ & $<0.001$ \\
\hline & Unknown & $7(4-10)$ & $5(3-10)$ & 0.20 \\
\hline \multirow[t]{5}{*}{$1 \mathrm{~h}$} & AMI & $68(32-193)$ & $88(34-164)$ & 0.49 \\
\hline & Unstable angina & $12(7-17)$ & $13(7-16)$ & 0.52 \\
\hline & Cardiac cause not $C A D^{b}$ & $15(7-30)$ & $10(6-27)$ & 0.07 \\
\hline & Non-cardiac cause & $6(4-10)$ & $5(4-8)$ & 0.004 \\
\hline & Unknown & $7(4-10)$ & $6(4-10)$ & 0.34 \\
\hline \multicolumn{5}{|c|}{ hs-cTn/Siemens, ng/L } \\
\hline \multirow[t]{5}{*}{$\mathrm{Oh}$} & AMI & $199(27-1270)$ & $158(22-559)$ & 0.35 \\
\hline & Unstable angina & $6(3-14)$ & $7(4-17)$ & 0.55 \\
\hline & Cardiac cause not $C A D^{b}$ & $9(3-50)$ & $6(2-24)$ & 0.45 \\
\hline & Non-cardiac cause & $2(1-5)$ & $2(1-5)$ & 0.09 \\
\hline & Unknown & $3(2-7)$ & $2(1-4)$ & 0.17 \\
\hline \multirow[t]{5}{*}{$1 \mathrm{~h}$} & AMI & $275(46-1357)$ & 203 (55-974) & 0.58 \\
\hline & Unstable angina & $6(4-15)$ & $8(4-17)$ & 0.66 \\
\hline & Cardiac cause not $C A D^{b}$ & $10(3-70)$ & $6(3-23)$ & 0.21 \\
\hline & Non-cardiac cause & $3(1-5)$ & $2(1-6)$ & 0.36 \\
\hline & Unknown & $4(2-7)$ & $3(1-4)$ & 0.22 \\
\hline \multicolumn{5}{|c|}{$h s-c T n / A b b o t t, n g / L$} \\
\hline \multirow[t]{5}{*}{ Oh } & AMI & $114(22-628)$ & $107(21-424)$ & 0.82 \\
\hline & Unstable angina & $6(3-12)$ & $8(4-13)$ & 0.49 \\
\hline & Cardiac cause not $C A D^{b}$ & $9(4-32)$ & $7(3-18)$ & 0.46 \\
\hline & Non-cardiac cause & $3(2-5)$ & $3(2-5)$ & 0.39 \\
\hline & Unknown & $3(3-5)$ & $3(2-4)$ & 0.44 \\
\hline \multirow[t]{5}{*}{$1 \mathrm{~h}$} & AMI & $151(28-871)$ & 217 (38-613) & 0.52 \\
\hline & Unstable angina & $6(4-12)$ & $9(4-14)$ & 0.46 \\
\hline & Cardiac cause not $C A D^{b}$ & $10(3-50)$ & $7(4-15)$ & 0.40 \\
\hline & Non-cardiac cause & $3(2-5)$ & $3(2-6)$ & 0.58 \\
\hline & Unknown & $3(2-5)$ & $3(2-3)$ & 0.85 \\
\hline
\end{tabular}

${ }^{a}$ Compared with the Mann Whitney $U$ test. ${ }^{b} \mathrm{CAD}$; coronary artery disease 
Early-morning

A

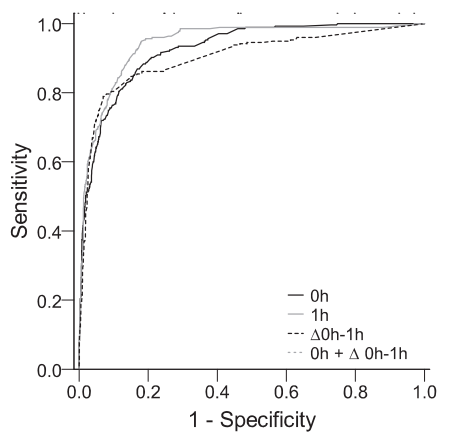

B

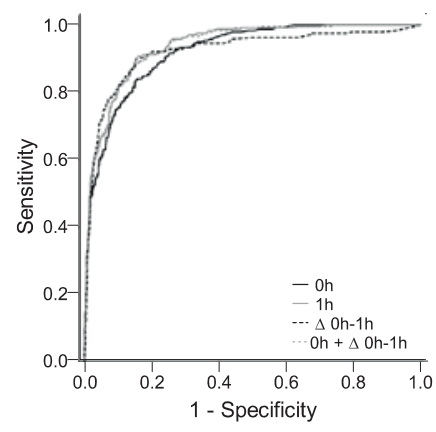

C

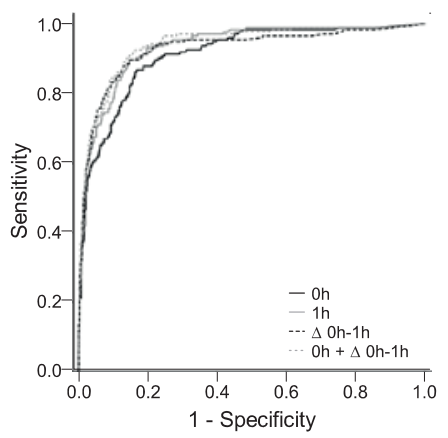

Evening
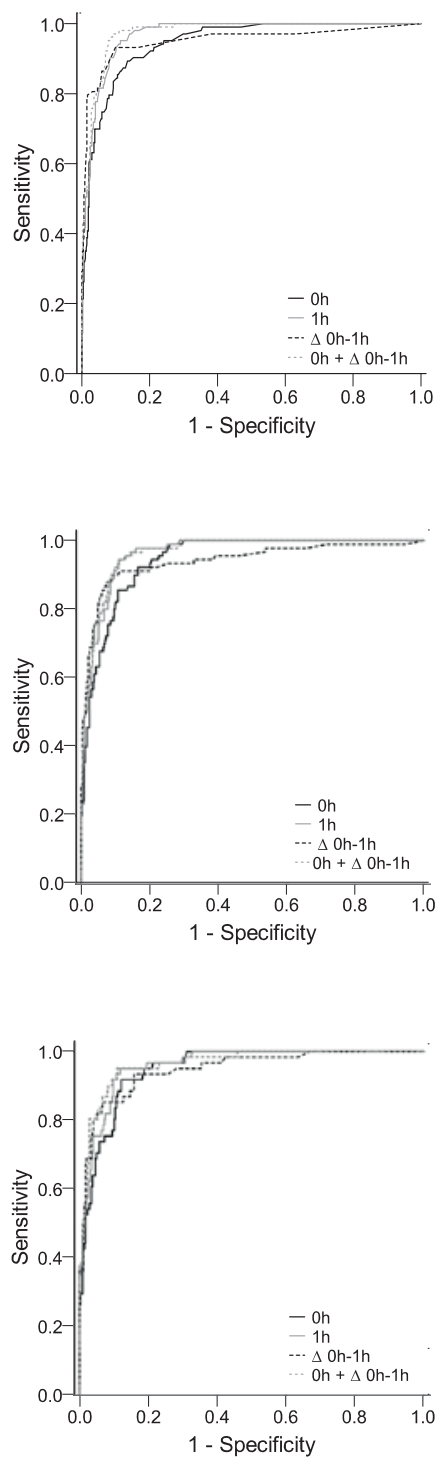

Figure 2. Diagnostic performance of hs-cTnT and hs-cTnl for AMl in early-morning and evening presenters. ROC curves show the diagnostic accuracy of hs-cTnT (A) and hs-cTnl (B (Abbott) and C (Siemens)) for AMl in patients presenting in the early morning (11.00 PM-2.00 PM) and in the evening (2.00 PM-11.00 PM). 
Table 3. Diagnostic accuracy of cTnT and cTnl in early-morning and evening presenters

\begin{tabular}{|c|c|c|c|}
\hline & Early-morning & Evening & \\
\hline & $11.00 \mathrm{PM}-2.00 \mathrm{PM}$ & 2.00 PM - 11.00 PM & \\
\hline & $\operatorname{AUC}(95 \% \mathrm{Cl})$ & $\operatorname{AUC}(95 \% \mathrm{Cl})$ & p-value \\
\hline \multicolumn{4}{|c|}{ hs-cTnT Roche, $n g / L$} \\
\hline $\mathrm{Oh}$ & $0.93(0.92-0.94)$ & $0.94(0.92-0.96)$ & 0.58 \\
\hline $1 \mathrm{~h}$ & $0.94(0.93-0.95)$ & $0.97(0.95-0.98)$ & 0.10 \\
\hline$\Delta 1 \mathrm{~h}$ & $0.90(0.89-0.92)$ & $0.95(0.93-0.97)$ & 0.010 \\
\hline $0 h+\Delta 1 h$ & $0.96(0.95-0.97)$ & $0.98(0.96-0.99)$ & 0.20 \\
\hline \multicolumn{4}{|c|}{ hs-cTn/Siemens, $n g / L$} \\
\hline $\mathrm{Oh}$ & $0.92(0.90-0.93)$ & $0.94(0.92-0.96)$ & 0.32 \\
\hline $1 \mathrm{~h}$ & $0.93(0.92-0.95)$ & $0.96(0.94-0.98)$ & 0.24 \\
\hline$\Delta 1 \mathrm{~h}$ & $0.93(0.91-0.94)$ & $0.95(0.92-0.97)$ & 0.39 \\
\hline $0 h+\Delta 1 h$ & $0.94(0.92-0.95)$ & $0.96(0.94-0.98)$ & 0.26 \\
\hline \multicolumn{4}{|c|}{$h s-c T n / A b b o t t, n g / L$} \\
\hline $\mathrm{Oh}$ & $0.93(0.91-0.94)$ & $0.94(0.92-0.96)$ & 0.50 \\
\hline $1 \mathrm{~h}$ & $0.94(0.91-0.94)$ & $0.96(0.94-0.98)$ & 0.09 \\
\hline$\Delta 1 \mathrm{~h}$ & $0.92(0.91-0.94)$ & $0.94(0.92-0.96)$ & 0.35 \\
\hline $0 h+\Delta 1 h$ & $0.94(0.92-0.95)$ & $0.97(0.95-0.98)$ & 0.11 \\
\hline
\end{tabular}

Table 4. Diagnostic accuracy of cTnT and cTnl in early-morning and evening presenters ( $\leq 3 \mathrm{~h}$ since symptom onset)

\begin{tabular}{|c|c|c|c|}
\hline & Early-morning & Evening & \\
\hline & $11.00 \mathrm{PM}-2.00 \mathrm{PM}$ & $2.00 \mathrm{PM}-11.00 \mathrm{PM}$ & \\
\hline & $\operatorname{AUC}(95 \% \mathrm{Cl})$ & $\operatorname{AUC}(95 \% \mathrm{Cl})$ & p-value \\
\hline \multicolumn{4}{|c|}{ hs-cTnT Roche, ng/L } \\
\hline $\mathrm{Oh}$ & $0.90(0.89-0.92)$ & $0.93(0.91-0.96)$ & 0.26 \\
\hline $1 \mathrm{~h}$ & $0.94(0.92-0.95)$ & $0.98(0.96-0.99)$ & 0.050 \\
\hline$\Delta 1 \mathrm{~h}$ & $0.90(0.88-0.92)$ & $0.95(0.93-0.97)$ & 0.01 \\
\hline $0 h+\Delta 1 h$ & $0.95(0.93-0.96)$ & $0.98(0.96-0.99)$ & 0.06 \\
\hline \multicolumn{4}{|c|}{ hs-cTn/ Siemens, ng/L } \\
\hline $\mathrm{Oh}$ & $0.91(0.88-0.93)$ & $0.94(0.90-0.96)$ & 0.36 \\
\hline $1 \mathrm{~h}$ & $0.94(0.92-0.96)$ & $0.96(0.93-0.98)$ & 0.63 \\
\hline$\Delta 1 \mathrm{~h}$ & $0.93(0.91-0.94)$ & $0.95(0.92-0.97)$ & 0.41 \\
\hline $0 h+\Delta 1 h$ & $0.95(0.93-0.97)$ & $0.97(0.94-0.98)$ & 0.61 \\
\hline \multicolumn{4}{|c|}{$h s-c T n / A b b o t t, n g / L$} \\
\hline $\mathrm{Oh}$ & $0.92(0.90-0.94)$ & $0.94(0.91-0.97)$ & 0.42 \\
\hline $1 \mathrm{~h}$ & $0.94(0.92-0.96)$ & $0.97(0.94-0.99)$ & 0.25 \\
\hline$\Delta 1 \mathrm{~h}$ & $0.91(0.90-0.94)$ & $0.94(0.92-0.96)$ & 0.20 \\
\hline $0 h+\Delta 1 h$ & $0.94(0.92-0.96)$ & 0.97 (0.94-0.99) & 0.19 \\
\hline
\end{tabular}




\section{Effect on sensitivity, specificity, positive predictive value and negative predictive value}

Using the ESC2015 NSTEMI cutoff values ${ }^{1}$, specificity of hs-cTnT, but not hs-cTnl, is slightly lower in the early morning compared to the evening. (Supplementary Table 7) However, inclusion of the $1 \mathrm{~h}$ delta-change value, which is the most important determinant to increase specificity in clinical practice, neutralizes the specificity difference between morning and evening presenters, hence limiting the clinical relevance of the baseline difference. Negative and positive predictive value of hs-cTnT are also similar in early-morning and evening presenters (Supplementary Table 7).

\section{Discussion}

The aim of the present study was to validate the diurnal troponin rhythm as a general phenomenon and to evaluate whether diurnal oscillation affect the diagnostic accuracy for AMI. We report seven major findings:

First, we identified rhythmic diurnal hs-cTnT variation as a general phenomenon, characterized by gradually declining levels during daytime followed by rising concentrations during nighttime to peak concentrations at again (early) morning hours. The rhythm is irrespective of sex and glucose metabolism status, with the largest amplitude of the oscillation seen in stable subjects with the highest hs-cTnT concentrations. Second, the diurnal rhythm is restricted to hs-cTnT, with no evidence for rhythmic variation detected in hs-cTnl and sensitive cTnl as quantified by three different cTnl assays.cTnT (36 kDa) and cTnl $(24 \mathrm{kDa})$ are components of the thin filament of cardiomyocytes with possibly a small proportion existing as unbound protein in the cytosol ${ }^{18,19}$. Release of cTn in the circulation is strongly associated with myocardial injury, although it is unclear whether detectable levels in healthy individuals reflect protein turnover or cardiomyocyte apoptosis ${ }^{6}$. As measured in serum from AMI patients, circulating cTn consists of intact and degradation forms that are ultimately cleared by the kidneys ${ }^{20}$. We can only speculate which physiological differences between cTnT and cTnl underlie the observed differences in the diurnal rhythmicity. Renal function may play a role, as various observations suggests that deteriorating renal function is stronger related to elevated cTnT levels than cTnl levels ${ }^{21-25}$. However, it is unknown 
whether diurnal variations in renal function would be sufficient to cause rather pronounced relative changes in hs-cTnT in the normal range. Accordingly, differences in cTn metabolism including repair mechanisms may also play a role. Third, these results could be reproduced in the large APACE study: significantly higher hs-cTnT levels were evident in early-morning presenters compared to evening presenters with an adjudicated final diagnosis of noncardiac disease. Fourth, diagnostic accuracy of hs-cTnT for AMI overall was very high in patients presenting in the early-morning as well as patients presenting in the evening and not significantly different between the two. Fifth, the physiological rise and fall of hs-cTnT during the day significantly affected the diagnostic accuracy of absolute changes within the first hour after presentation with lower AUC in the early-morning (when non-cardiac disease and healthy persons have higher levels) as compared to the evening (when non-cardiac disease and healthy persons have lower levels). This result could not be reproduced in changes within 2, 3 and 6h. As in current clinical practice absolute changes are not used as a primary diagnostic variable, but only secondary to the absolute level at presentation and at $1 \mathrm{~h}$, or in conjunction with the absolute level at presentation/1h, the clinical impact - if any of the circadian rhythm in hs-cTnT seems to be very small overall. Sixth, in patients presenting very early $(<3 \mathrm{~h})$ after chest pain onset, the difference in AUC seemed to be more pronounced. This observation can be well explained that in early presenters the true circadian baseline level has still a stronger impact on hs-cTnT blood concentrations in AMI, as the added hs-cTnT component resulting exclusively from the AMI event is much smaller very early versus later after chest pain onset. As the magnitude of the difference in AUC was small, the clinical relevance of this difference seems to be very small at most also in early presenters. The circadian rhythm in hs-cTnT may well however affect the diagnostic accuracy of hs-cTnT when used as a screening tool for cardiovascular disease, where much smaller differences exist between health and disease. Accordingly, screening with its need for serial testing over years should be scheduled on the same time of the day in the individual patient. Seventh, prognostic accuracy of hs-cTnT for all-cause mortality within one year was moderate-to-high and not affected by the circadian rhythm. 
Our findings extend and corroborate recent pilot data suggesting a diurnal rhythm for hscTnT in a small group of male subjects with type 2 diabetes ${ }^{12}$. As the circulating hs-cTn concentration is defined by the extent of release and clearance, this study does not allow to quantify the influence of both processes on the diurnal hs-cTnT rhythm. Nevertheless, the rhythmicity of this cardiac biomarker is in line with the circadian organization of the cardiovascular system ${ }^{26,27}$. Synchronizing physiological functions to the natural $24 \mathrm{~h}$ light-dark cycle, for example by upregulating body temperature and blood pressure before the time of awakening, anticipates to the demands of daytime activities. Circadian rhythmicity has in addition been described for many cardiovascular traits: heart rate, sympathetic activity, vascular resistance, the renin-angiotensin-aldosteron system, prothrombotic tendency, platelet aggregability and the fibrinolytic system ${ }^{27}$. Identical to hs-cTnT concentrations, all these components exhibit peak activity in (early) morning hours. The physiological rhythmicity of these cardiovascular components is reflected in the increasing intensity of cardiovascular events in the morning hours. For example, the incidence of AMI, sudden cardiac death and stroke display a significant $24 \mathrm{~h}$ variation, characterized by a higher frequency in morning hours ${ }^{28}$. Even the clock time of AMI onset is associated with clinical course and survival, with the largest infarct sizes and higher mortality found among patients with $\mathrm{AMI}$ that commence in morning hours ${ }^{29,30}$.

Potential limitations of our study merit consideration. First, study 1 enrolled 19 men compared to 5 women, which is a limitation given the difference in clinical hs-reference values between both genders. Second, study 2 was conducted with patients presenting to the emergency department with symptoms suggestive of AMI. Additional studies in patients presenting to a general practitioner, which in general have a much lower pretest-probability for AMI, are required to learn whether the diurnal hs-cTnT rhythm affects the diagnostic accuracy in this setting. Third, this diagnostic study required written informed consent. Therefore, invariably the rate of enrollment was lower than in registries. Fourth, we cannot comment on diurnal rhythms in patients with terminal kidney failure requiring dialysis, since such patients were excluded from our study. 
In conclusion, rhythmic diurnal variation of hs-cTnT is a general phenomenon, that is not seen with hs-cTnl, and should trigger additional studies to better understand its pathophysiology. Fortunately, the rhythmic diurnal variation of hs-cTnT does not seem to affect the high diagnostic accuracy of hs-cTnT in the diagnosis of AMI. It should however be considered when using hs-cTnT as a screening tool, where much smaller differences exist between health and disease. 


\section{References}

1. Roffi M, Patrono C, Collet JP, et al. 2015 ESC Guidelines for the management of acute coronary syndromes in patients presenting without persistent ST-segment elevation: Task Force for the Management of Acute Coronary Syndromes in Patients Presenting without Persistent ST-Segment Elevation of the European Society of Cardiology (ESC). European heart journal 2016;37:267-315.

2. Rubini Gimenez M, Twerenbold R, Reichlin T, et al. Direct comparison of high-sensitivity-cardiac troponin I vs. T for the early diagnosis of acute myocardial infarction. European heart journal 2014;35:2303-11.

3. Keller T, Zeller T, Peetz D, et al. Sensitive troponin I assay in early diagnosis of acute myocardial infarction. The New England journal of medicine 2009;361:868-77.

4. Reichlin T, Hochholzer W, Bassetti S, et al. Early diagnosis of myocardial infarction with sensitive cardiac troponin assays. The New England journal of medicine 2009;361:858-67.

5. Mills NL, Churchhouse AM, Lee KK, et al. Implementation of a sensitive troponin I assay and risk of recurrent myocardial infarction and death in patients with suspected acute coronary syndrome. Jama 2011;305:1210-6.

6. de Lemos JA. Increasingly sensitive assays for cardiac troponins: a review. Jama 2013;309:2262-9.

7. van der Linden $\mathrm{N}$, Tieland $\mathrm{M}$, Klinkenberg $L$, et al. The effect of a six-month resistance-type exercise training program on the course of high sensitive cardiac troponin T levels in (pre)frail elderly. International journal of cardiology 2014;175:374-5.

8. Giannitsis E, Katus HA. Cardiac troponin level elevations not related to acute coronary syndromes. Nat Rev Cardiol 2013;10:623-34.

9. Thygesen K, Alpert JS, Jaffe AS, et al. Third universal definition of myocardial infarction. European heart journal 2012;33:2551-67.

10. Hamm CW, Bassand JP, Agewall S, et al. ESC Guidelines for the management of acute coronary syndromes in patients presenting without persistent ST-segment elevation: The Task Force for the management of acute coronary syndromes (ACS) in patients presenting without persistent ST-segment elevation of the European Society of Cardiology (ESC). European heart journal 2011;32:2999-3054.

11. Thygesen K, Mair J, Giannitsis E, et al. How to use high-sensitivity cardiac troponins in acute cardiac care. European heart journal 2012;33:2252-7.

12. Klinkenberg LJ, van Dijk JW, Tan FE, van Loon L, van Dieijen-Visser MP, Meex SJ. Circulating cardiac troponin T exhibits a diurnal rhythm. Journal of the American College of Cardiology 2014;63:1788-95.

13. Haaf $P$, Reichlin $T$, Twerenbold $R$, et al. Risk stratification in patients with acute chest pain using three highsensitivity cardiac troponin assays. European heart journal 2014;35:365-75.

14. Reiter M, Twerenbold R, Reichlin T, et al. Early diagnosis of acute myocardial infarction in patients with preexisting coronary artery disease using more sensitive cardiac troponin assays. European heart journal 2012;33:98897.

15. Nelson W, Tong YL, Lee JK, Halberg F. Methods for cosinor-rhythmometry. Chronobiologia 1979;6:305-23.

16. Hanley JA, McNeil BJ. A method of comparing the areas under receiver operating characteristic curves derived from the same cases. Radiology 1983;148:839-43.

17. Dill DB, Costill DL. Calculation of percentage changes in volumes of blood, plasma, and red cells in dehydration. Journal of applied physiology 1974;37:247-8.

18. Katrukha IA. Human cardiac troponin complex. Structure and functions. Biochemistry (Mosc) 2013;78:1447-65.

19. Katus HA, Remppis A, Scheffold T, Diederich KW, Kuebler W. Intracellular compartmentation of cardiac troponin $T$ and its release kinetics in patients with reperfused and nonreperfused myocardial infarction. Am J Cardiol 1991;67:1360-7.

20. Cardinaels EP, Mingels AM, van Rooij T, Collinson PO, Prinzen FW, van Dieijen-Visser MP. Time-dependent degradation pattern of cardiac troponin T following myocardial infarction. Clinical chemistry 2013;59:1083-90.

21. Hickman PE, Koerbin G, Southcott E, et al. Newer cardiac troponin I assays have similar performance to troponin $T$ in patients with end-stage renal disease. Annals of clinical biochemistry 2007;44:285-9. 
22. deFilippi C, Seliger SL, Kelley W, et al. Interpreting cardiac troponin results from high-sensitivity assays in chronic kidney disease without acute coronary syndrome. Clinical chemistry 2012;58:1342-51.

23. Collinson PO, Heung YM, Gaze D, et al. Influence of population selection on the 99th percentile reference value for cardiac troponin assays. Clinical chemistry 2012;58:219-25.

24. Apple FS, Murakami MM, Pearce LA, Herzog CA. Predictive value of cardiac troponin I and T for subsequent death in end-stage renal disease. Circulation 2002;106:2941-5.

25. Jacobs LH, van de Kerkhof J, Mingels AM, et al. Haemodialysis patients longitudinally assessed by highly sensitive cardiac troponin T and commercial cardiac troponin T and cardiac troponin I assays. Annals of clinical biochemistry 2009;46:283-90.

26. Martino TA, Sole MJ. Molecular time: an often overlooked dimension to cardiovascular disease. Circulation research 2009;105:1047-61.

27. Sato M, Matsuo T, Atmore H, Akashi M. Possible contribution of chronobiology to cardiovascular health. Frontiers in physiology 2013;4:409.

28. Manfredini R, Boari B, Salmi R, et al. Twenty-four-hour patterns in occurrence and pathophysiology of acute cardiovascular events and ischemic heart disease. Chronobiology international 2013;30:6-16.

29. Reiter R, Swingen C, Moore L, Henry TD, Traverse JH. Circadian dependence of infarct size and left ventricular function after ST elevation myocardial infarction. Circulation research 2012;110:105-10.

30. Manfredini R, Boari B, Bressan S, et al. Influence of circadian rhythm on mortality after myocardial infarction: data from a prospective cohort of emergency calls. The American journal of emergency medicine 2004;22:555-9. 


\section{Supplemental information}

\section{Supplemental Methods}

\section{Adjudication of final diagnoses}

Adjudication of the final diagnosis was performed centrally in a core lab (University Hospital Basel)1-5 and included two sets of cardiac troponin (cTn) measurements. Those performed clinically in each hospital at presentation and at 3-6h and possibly also at 12-24h according to current guidelines ${ }^{6,7}$, as well as levels of high-sensitivity cardiac troponin T (hs-cTnT) measured from the study blood samples taken at presentation, at $1 \mathrm{~h}, 2 \mathrm{~h}, 3 \mathrm{~h}$, and $6 \mathrm{~h}$ (if feasible) in order to take advantage of the higher sensitivity and higher overall diagnostic accuracy offered by hs-cTnT (this allows the additional detection of small acute myocardial infarctions [AMIs] $]^{1,2,8}$.

AMI was defined and cTn levels interpreted as recommended in current guidelines ${ }^{6,7,9}$. In brief, AMI was diagnosed when there was evidence of myocardial necrosis in association with a clinical setting consistent with myocardial ischemia. Myocardial necrosis was diagnosed by at least one cTn value above the $9^{\text {th }}$ percentile together with a significant rising and/or falling6,7,9. All other patients were classified as 'NO NSTEMI' for this analysis, including in this group the categories of unstable angina (UA), non-cardiac chest pain (NCCP), cardiac but non-coronary disease (e.g. tachyarrhythmia, perimyocarditis), and symptoms of unknown origin with normal levels of cTn and hs-cTnT.

The following conventional cTn assays were used: For the Roche CTnT $4^{\text {th }}$ generation assay, the $10 \% \mathrm{CV}$ level is $0.035 \mu \mathrm{g} / \mathrm{L}$. The laboratories of the participating sites reported only two decimals; therefore $0.04 \mu \mathrm{g} / \mathrm{L}$ was used as a cut-off for myocardial necrosis. In order to fulfil the criteria of a significant change ( $30 \%$ of $99^{\text {th }}$ percentile or $10 \% \mathrm{CV}$ level), a patient would e.g. need to have a level of $<0.01 \mu \mathrm{g} / \mathrm{L}$ at presentation and $0.04 \mu \mathrm{g} / \mathrm{L}$ at $6 \mathrm{~h}$. A patient would also qualify if the first level is $0.02 \mu \mathrm{g} / \mathrm{L}$ and the second $0.04 \mu \mathrm{g} / \mathrm{L}$. A patient would not fulfil the criteria if the first level is $0.03 \mu \mathrm{g} / \mathrm{L}$ and the second is $0.04 \mu \mathrm{g} / \mathrm{L}$. If the first level is $0.04 \mu \mathrm{g} / \mathrm{L}$, the second level needs to be at least $0.06 \mu \mathrm{g} / \mathrm{L}$. For the Abbott Axsym cTnI ADV, the $10 \% \mathrm{CV}$ level is $0.16 \mu \mathrm{g} / \mathrm{L}$. A patient having $0.16 \mu \mathrm{g} / \mathrm{L}$ at presentation would meet the criteria for significant change if the second was $\geq 0.21 \mu \mathrm{g} / \mathrm{L}$. A patient having $<0.12 \mu \mathrm{g} / \mathrm{L}$ at presentation (limit of detection) would qualify if the second is $>0.16 \mu \mathrm{g} / \mathrm{L}$. For the Beckmann Coulter Accu CTnl, the $10 \% \mathrm{CV}$ level is $0.06 \mu \mathrm{g} / \mathrm{L}$. A patient having $0.06 \mu \mathrm{g} / \mathrm{L}$ at presentation would qualify if the second is $\geq 0.08 \mu \mathrm{g} / \mathrm{L}$. A patient having $0.05 \mu \mathrm{g} / \mathrm{L}$ at presentation would qualify if the second is 0.07 $\mu \mathrm{g} / \mathrm{L}$, but not $0.06 \mu \mathrm{g} / \mathrm{L}$. A patient having undetectable $\mathrm{cTnl}(\mathrm{cTn} \mid<0.01 \mu \mathrm{g} / \mathrm{L})$ at presentation would qualify if the second is $\geq 0.06 \mu \mathrm{g} / \mathrm{L}$. 
For hs-cTnT, the 99th percentile ( $14 \mathrm{ng} / \mathrm{L}$ ) was used as cut-off for myocardial necrosis. Absolute changes in hs-cTnT were used to determine significant changes based on the diagnostic superiority of absolute over relative changes. Based on studies of the biological variation of CTn as well as on data from previous chest pain cohort studies, a significant absolute change was defined as a rise or fall of at least $10 \mathrm{ng} / \mathrm{L}$ within six hours. In patients, in whom a 6 hour hs-cTnT level was not available, changes were assessed at earlier time points. In an assumption of linearity, an absolute change of $6 \mathrm{ng} / \mathrm{L}$ within three hours was considered.

\section{Follow up}

After hospital discharge, patients were contacted after 3, 12 and 24 months by telephone calls or in written form. Information regarding death was furthermore obtained from the national registry on mortality, the hospitals' diagnosis registry, and the family physicians' records.

\section{Laboratory analysis}

cTnT was measured by using the high-sensitivity cTnT assay of Roche Diagnostics (Cobas 8000); the 99th percentile among healthy subjects is $14 \mathrm{ng} / \mathrm{L}$, with a $10 \%$ analytical variation at $13 \mathrm{ng} / \mathrm{L}^{10}$. Abbott highsensitivity cTnl was measured with the STAT high-sensitivity troponin I assay (Architect, Abbott Diagnostics). According to the manufacturer, the $99^{\text {th }}$ percentile is $26.2 \mathrm{ng} / \mathrm{L}$ with a corresponding coefficient of variation of $<5 \%$. The high-sensitivity cTnl assay of Siemens has a $99^{\text {th }}$ percentile concentration of $9 \mathrm{ng} / \mathrm{L}$ and a coefficient of variation of $10 \%$ at $3 \mathrm{ng} / \mathrm{L}$ (according to the manufacturer). S-cTnl (Beckman Coulter) was measured by using the Access AccuTnl+3 Assay; with a 99th percentile limit of $40 \mathrm{ng} / \mathrm{L}$ with a corresponding 10\% imprecision, as specified by the manufacturer. Cortisol, thyroid-stimulating hormone and testosterone were measured by using the assays of Roche Diagnostics (Cobas 8000). 


\section{Supplemental Figures}

A

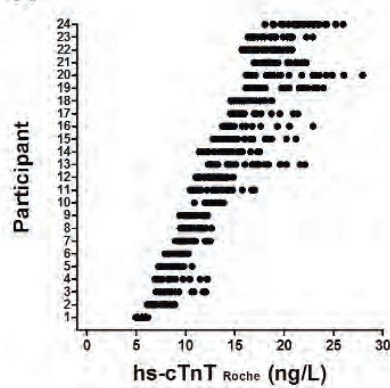

B

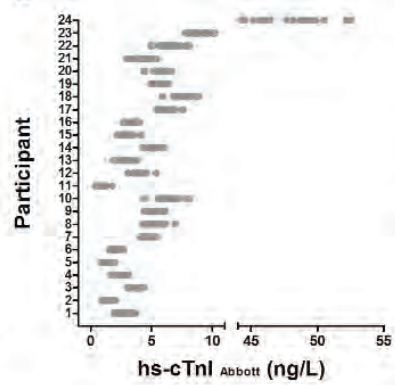

C

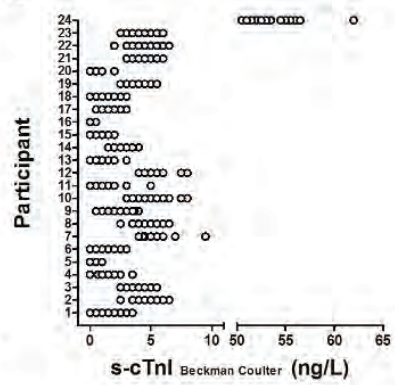

Supplemental figure 1. 25h distribution of high-sensitivity cardiac troponin T and I. Distribution of high sensitivity cardiac troponin T (hs-cTnT Roche Diagnostics) (A), high sensitivity cardiac troponin I (hs-cTnl Abbott Diagnostics) (B), and sensitive cardiac troponin I (s-cTnI Beckman Coulter) (C) in 24 subjects (8.30 AM to 9.30 AM the next day). Circles represent mean troponin values from each sampling point for a given individual. Participants 1, 2, 8, 12 and 21 are female subjects. Subjects were ranked on the basis of their visual range of hs-cTnT concentration: generally, high subject number correspond to a wider range in hs-cTnT concentration. The ranking used for Supplemental figure $1 \mathrm{~A}$ is extended to Supplemental figures $1 \mathrm{~B}, 1 \mathrm{C}$, Table 1, Supplemental figure 2, 5, 6, allowing direct comparisons throughout the paper. 

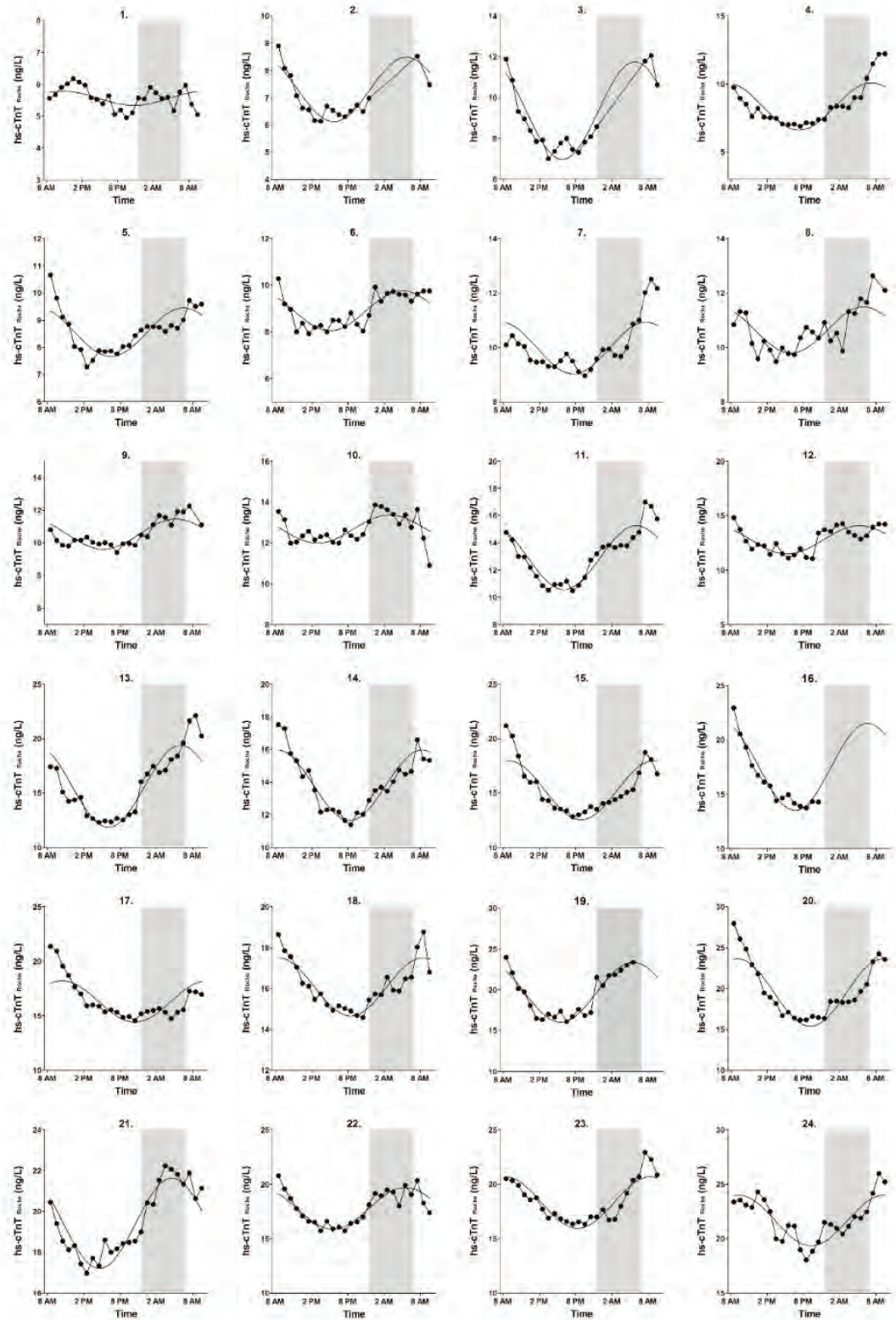

Supplemental figure 2. Individual diurnal high-sensitivity cardiac troponin T profiles and fitted cosine curves. Depicted are the mean of the duplicate measurements. The numbering is identical to Table 1, Supplemental figure 1, 5, 6 allowing direct comparisons throughout the paper. Subjects slept in a supine position between 11.30 PM and 7.00 AM (shaded area). Sampling in subjects 2, 3 and 16 was hindered throughout the night due to technical problems with the intravenous cannula. 


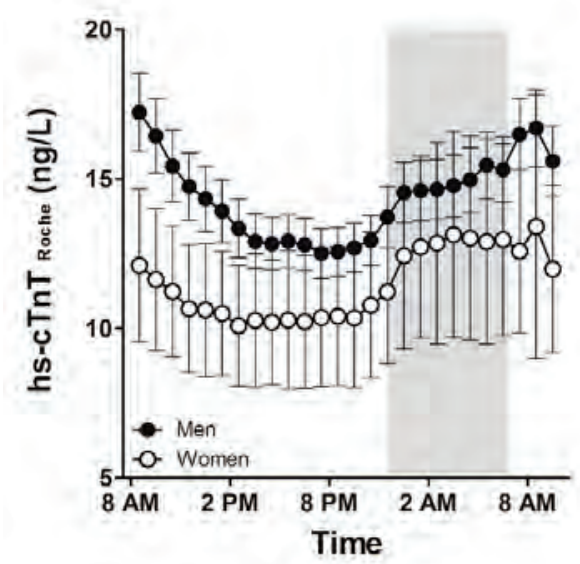

Supplemental figure 3. Mean \pm SEM $25 \mathrm{~h}$ high-sensitivity cardiac troponin T profile of men and women. Subjects slept in a supine position between 11.30 PM-7.00 AM (shaded area).

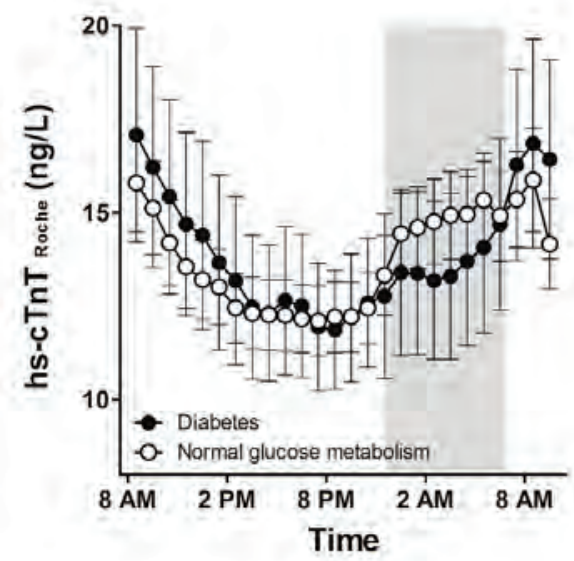

Supplemental figure 4. Mean \pm SEM 25h high-sensitivity cardiac troponin T profile of individuals with diabetes and normal glucose metabolism. Subjects slept in a supine position between 11.30 PM-7.00 AM (shaded area). 


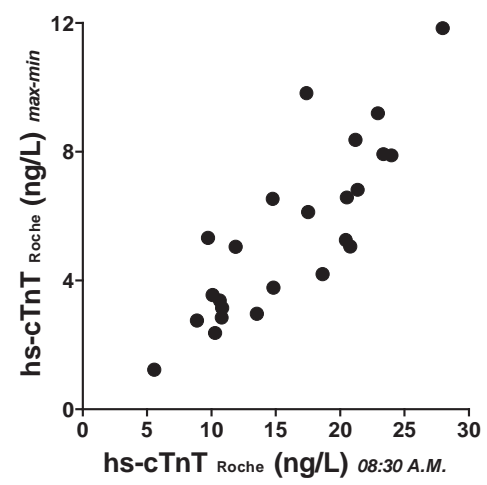

Supplemental figure 5. High-sensitivity cardiac troponin T fluctuations are most prominent in subjects with the highest troponin T concentrations. Correlation between the high sensitivity cardiac troponin T (hs-cTnT) value at 8.30 AM day 1 and the maximal intra-individual changes during the 25h test period (Pearson's R2 0.70; $p<0.001$ ).

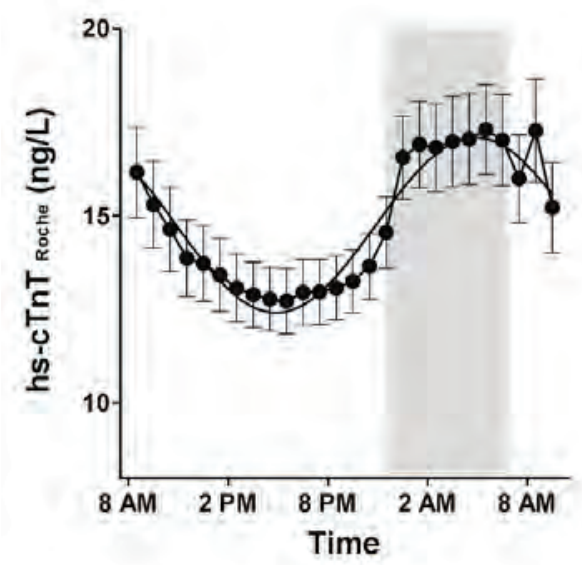

Supplemental figure 6. Mean diurnal high sensitivity cardiac troponin T profile corrected for possible plasma volume changes and fitted cosine curve. Depicted are the mean \pm SEM 25h high sensitivity cardiac troponin T (hs-cTnT) profile corrected for hemoglobin and hematocrit and fitted cosine curve. Subjects slept in a supine position between 11.30 PM and 7.00 AM (shaded area). The cosine curve is described as $Z(t)=14.75+2.34 \cdot \cos (15 t-305.56)$, with a R2 goodness of fit of $0.94(p<0.001)$. 
A
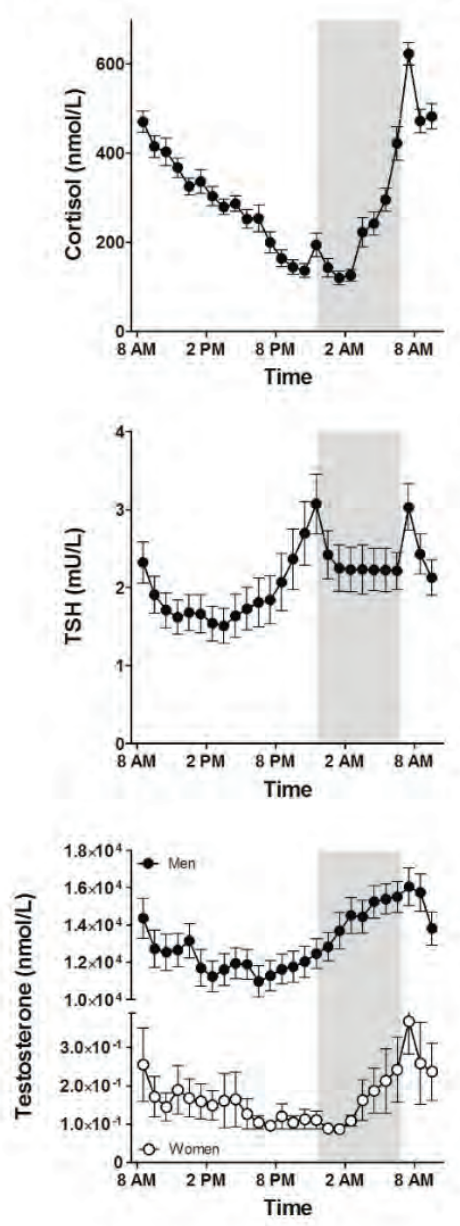

B
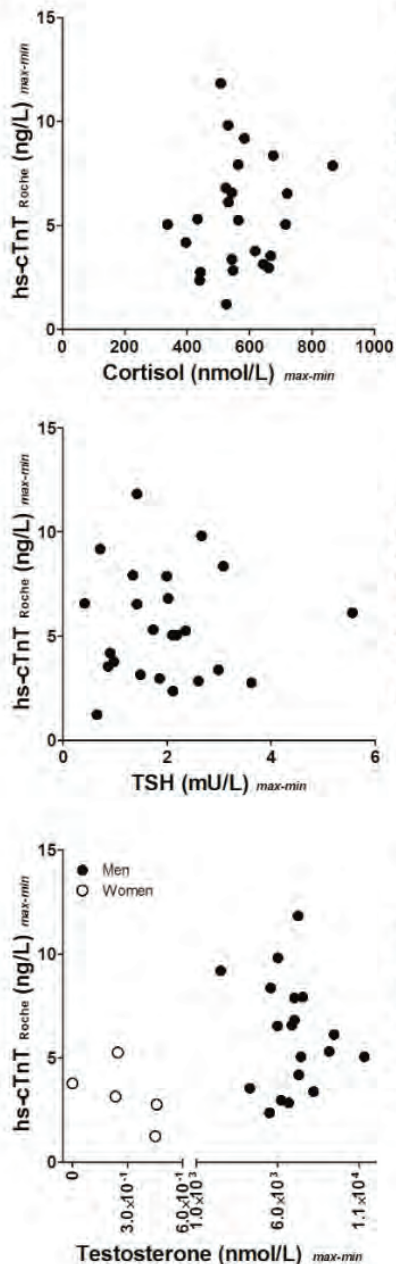

C
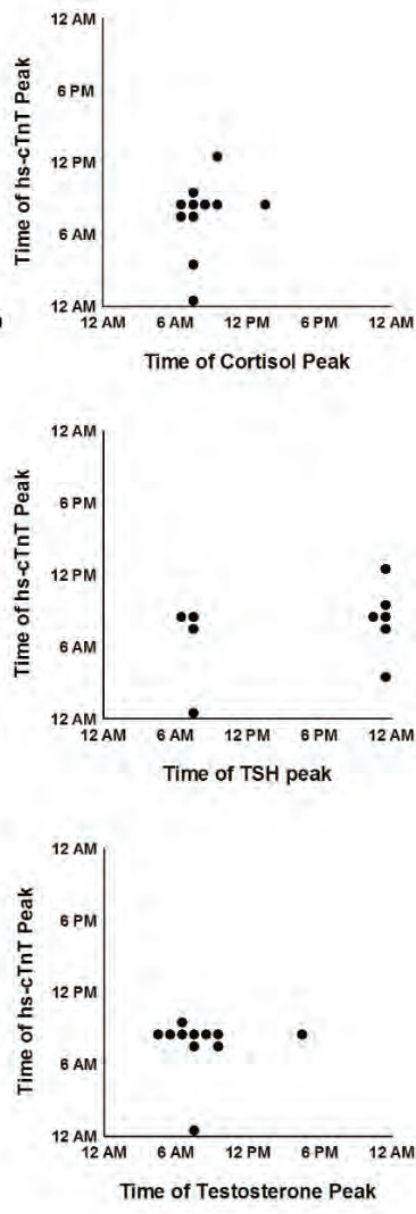

Supplemental figure 7. Relation of the diurnal profile of high-sensitivity cardiac troponin T with cortisol, thyroid stimulating hormone and testosterone. A) Mean $25 \mathrm{~h} \pm$ SEM profiles of cortisol, thyroid stimulating hormone (TSH) and testosterone. Subjects slept in a supine position between $11.30 \mathrm{PM}$ and 7.00 AM (shaded area). B) Relation between the maximal intra-individual changes of high-sensitivity cardiac troponin T (hs-cTnT) with cortisol, thyroidstimulating hormone (TSH) and testosterone. All correlations are non-significant. C) Relation between the time of high-sensitivity cardiac troponin T (hs-cTnT) peak with the time of cortisol, thyroid stimulating hormone (TSH) and testosterone. All correlations are non-significant. 

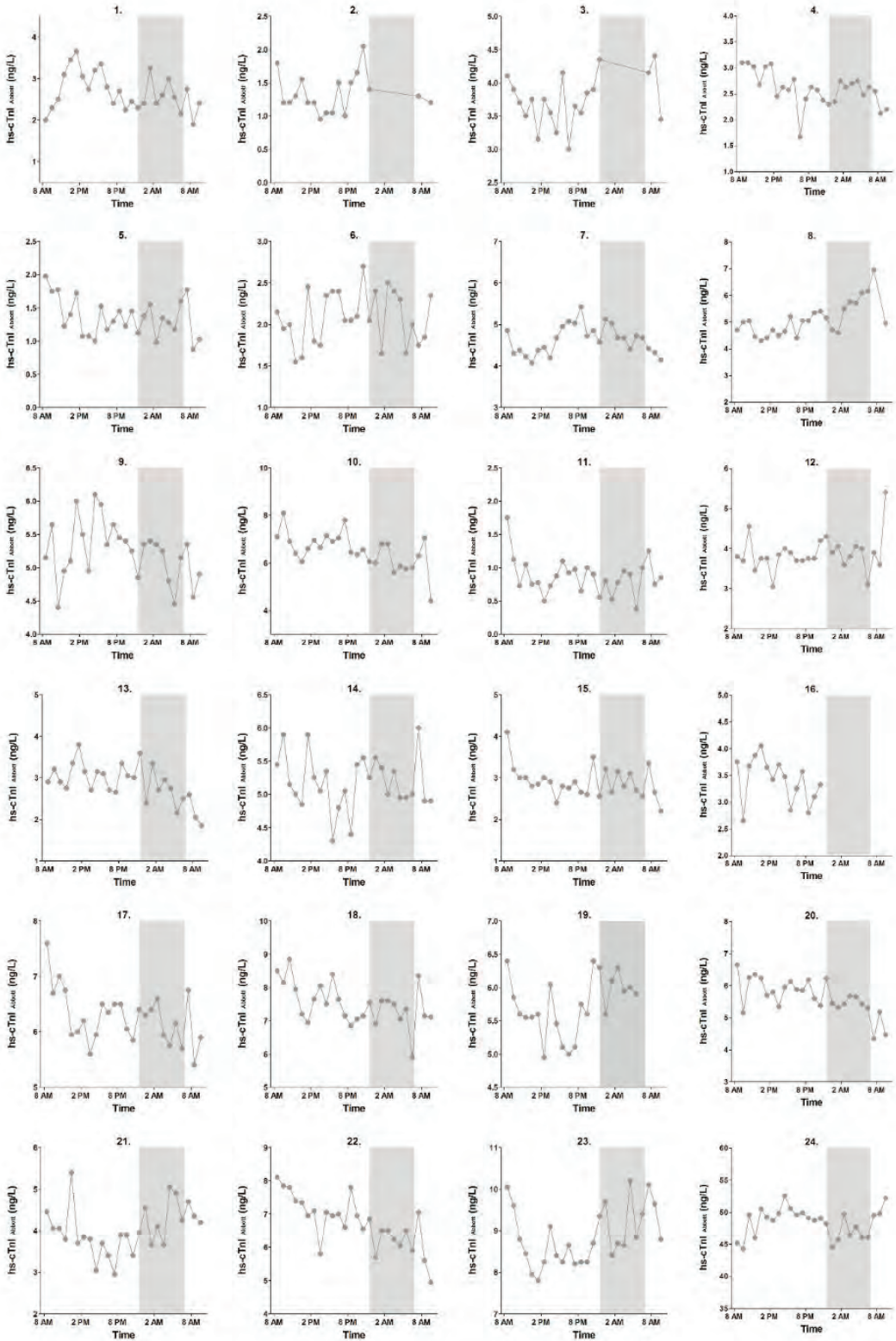

Supplemental figure 8. Individual diurnal high sensitivity cardiac troponin I profiles. Depicted are the mean of the duplicate measurements. The numbering is identical to Table 1, Supplemental figure 1, 2, 6, allowing direct comparisons throughout the paper. Subjects slept in a supine position between 11.30 PM and 7.00 AM (shaded area). Sampling in subjects 2, 3 and 16 was hindered throughout the night due to technical problems with the intravenous cannula. 

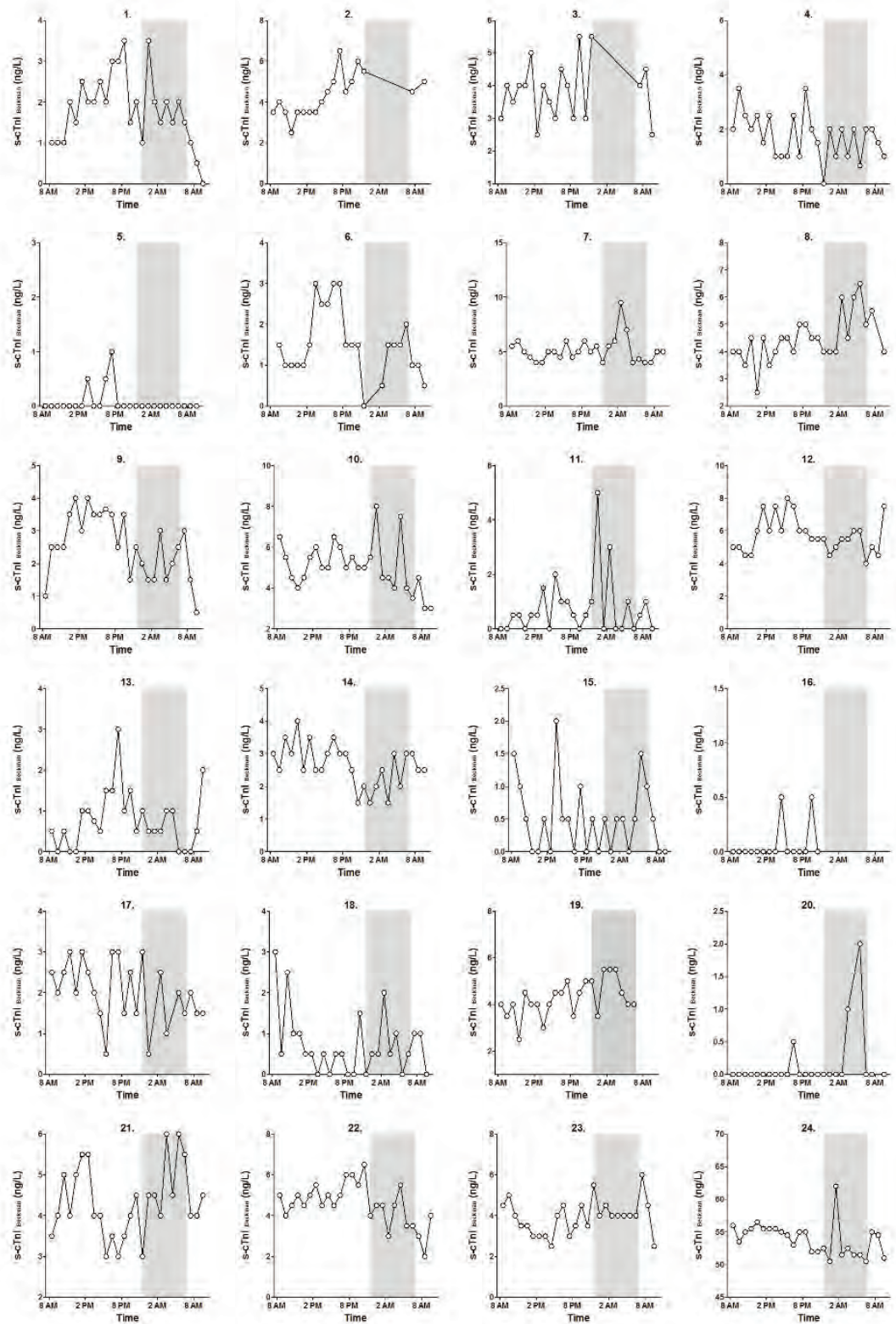

Supplemental figure 9. Individual diurnal sensitive cardiac troponin I profiles. Depicted are the mean of the duplicate measurements. The numbering is identical to Table 1 and Supplemental figures 1, 2 and 5, allowing direct comparisons throughout the paper. Subjects slept in a supine position between 11.30 PM and 7.00 AM (shaded area). Sampling in subjects 2, 3 and 16 was hindered throughout the night due to technical problems with the intravenous cannula. 


\section{Supplemental Tables}

Supplemental table 1. Study 1: Participants' characteristics

\begin{tabular}{|c|c|}
\hline & Study population \\
\hline$n$ & 24 \\
\hline Male gender & $19(79 \%)$ \\
\hline Age, years & $72 \pm 7$ \\
\hline Type 2 Diabetes Mellitus ${ }^{\mathrm{a}}$ & $7(29 \%)$ \\
\hline $\mathrm{BMI}, \mathrm{kg} / \mathrm{m}^{2}$ & $27 \pm 5$ \\
\hline Systolic blood pressure ${ }^{b}, \mathrm{~mm} \mathrm{Hg}$ & $140 \pm 15$ \\
\hline Diastolic blood pressure ${ }^{b}, \mathrm{~mm} \mathrm{Hg}$ & $68 \pm 8$ \\
\hline Fasting glucose ${ }^{\mathrm{b}}, \mathrm{mmol} / \mathrm{L}$ & $5.9(5.2-6.7)$ \\
\hline $\mathrm{HbA} 1 c^{b}, \%$ & $5.7(5.3-6.5)$ \\
\hline Total cholesterol ${ }^{\mathrm{b}}, \mathrm{mmol} / \mathrm{L}$ & $3.7(4.8-5.4)$ \\
\hline HDL cholesterol ${ }^{\mathrm{b}}, \mathrm{mmol} / \mathrm{L}$ & $1.3(1.6-1.9)$ \\
\hline LDL cholesterol ${ }^{\mathrm{b}}, \mathrm{mmol} / \mathrm{L}$ & $1.7(2.5-3.0)$ \\
\hline Triglycerides ${ }^{b}, \mathrm{mmol} / \mathrm{L}$ & $1.2(0.8-1.8)$ \\
\hline NT-proBNPb ${ }^{\mathrm{b}}$ pmol/L & $10.9(4.9-38.8)$ \\
\hline Hs-cTnT - Roche ${ }^{b}, \mathrm{ng} / \mathrm{L}$ & $16.1(10.7-21.1)$ \\
\hline Hs-cTnl-Abbottb, ng/L & $4.6(3.0-7.0)$ \\
\hline cTnl-Beckman Coulter ${ }^{\mathrm{b}}, \mathrm{ng} / \mathrm{L}$ & $3.0(1.0-4.5)$ \\
\hline $\mathrm{eGFR}{ }^{\mathrm{bc}}, \mathrm{mL} / \mathrm{min} / 1.73 \mathrm{~m}^{2}$ & $75(61-92)$ \\
\hline Oral blood glucose-lowering medication ${ }^{d}$ & $7(29 \%)$ \\
\hline Lipid-lowering medication ${ }^{d}$ & $12(50 \%)$ \\
\hline Anti-hypertensive medication ${ }^{d}$ & $10(42 \%)$ \\
\hline
\end{tabular}

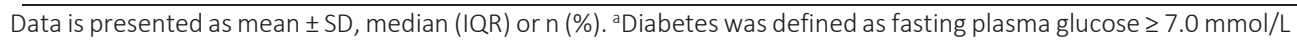
and/or $\mathrm{HbA} 1 \mathrm{c} \geq 6.5 \%{ }^{11}$. bValue at $8.30 \mathrm{AM}$ day $1 .{ }^{\mathrm{c}} \mathrm{eGFR}$, estimated glomerular filtration rate according to CKD-EPI Creatinine-Cystatin $C$ equation ${ }^{12}$. ${ }^{d}$ Medication status was stable for at least three months prior to inclusion, and continued as normal during the study period. To convert concentrations from $\mathrm{mmol} / \mathrm{L}$ to $\mathrm{mg} / \mathrm{dL}$, multiply glucose by 18.02 , cholesterol by 38.67 , and triglycerides by 88.54 . 
Supplemental table 2. Study 2: baseline characteristics of patients enrolled in the APACE trial.

\begin{tabular}{|c|c|c|c|c|}
\hline & All $(n=2782)$ & AMI $(n=488)$ & No AMI (n=2294) & p-value ${ }^{a}$ \\
\hline Age, $y$ & $61(49-74)$ & $72(59-80)$ & $59(47-72)$ & $<0.001$ \\
\hline Male gender, n (\%) & 1903 (68.4\%) & $360(73.8 \%)$ & $1543(67.3 \%)$ & 0.014 \\
\hline Body-mass index, $\mathrm{kg} / \mathrm{m}^{2}$ & $26(24-30)$ & $26(24-29)$ & $26(24-30)$ & 0.66 \\
\hline $\mathrm{eGFR}, \mathrm{mL} / \mathrm{min} / 1.73 \mathrm{~m}^{2}$ & $85(69-101)$ & $73(56-94)$ & $86(71-102)$ & $<0.001$ \\
\hline \multicolumn{5}{|l|}{ Risk factors, $n(\%)$} \\
\hline Hypertension & $1712(61.5)$ & $381(78.1)$ & $1331(58.0)$ & $<0.001$ \\
\hline Hypercholesterolemia & $1390(50.0)$ & $332(68.0)$ & $1058(46.1)$ & $<0.001$ \\
\hline Diabetes Mellitus & $477(17.1)$ & $126(25.8)$ & $351(15.3)$ & $<0.001$ \\
\hline Current or previous smoking & $1722(61.9)$ & $317(65.0)$ & $1405(61.2)$ & 0.11 \\
\hline Family history & 767 (27.6) & $158(32.4)$ & 609 (26.5) & 0.008 \\
\hline \multicolumn{5}{|l|}{ History, n (\%) } \\
\hline Coronary artery disease & $965(34.7)$ & $242(49.6)$ & $723(31.5)$ & $<0.001$ \\
\hline Previous AMI & $662(23.8)$ & $172(35.2)$ & $490(21.4)$ & $<0.001$ \\
\hline Previous revascularization & 775 (27.9) & $183(37.5)$ & $592(25.8)$ & $<0.001$ \\
\hline Peripheral artery disease & $169(6.1)$ & $63(12.9)$ & $106(4.6)$ & $<0.001$ \\
\hline Previous stroke & $152(5.5)$ & $44(9.0)$ & $108(4.7)$ & $<0.001$ \\
\hline \multicolumn{5}{|l|}{ ECG findings, $n(\%)$} \\
\hline Left bundle branch block & $79(2.8)$ & $28(5.7)$ & $51(2.2)$ & $<0.001$ \\
\hline ST-segment elevation & $57(2.0)$ & $13(2.7)$ & 44 (1.9) & 0.28 \\
\hline ST-segment depression & $282(10.1)$ & 146 (29.9) & $136(5.9)$ & $<0.001$ \\
\hline T-wave inversion & 359 (12.9) & $120(24.6)$ & $239(10.4)$ & $<0.001$ \\
\hline \multicolumn{5}{|l|}{ Medication at presentation, $n(\%)$} \\
\hline ASA/thienopyridine & $1111(39.9)$ & $268(54.9)$ & $843(36.7)$ & $<0.001$ \\
\hline b-Blockers & $966(34.7)$ & 214 (43.9) & $752(32.8)$ & $<0.001$ \\
\hline ACEIs/ARBs & $1069(38.4)$ & $253(51.8)$ & $816(35.6)$ & $<0.001$ \\
\hline Calcium antagonists & $411(14.8)$ & $100(20.5)$ & $311(13.6)$ & $<0.001$ \\
\hline
\end{tabular}

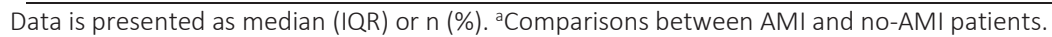


Supplemental table 3. Multivariate analysis of baseline high-sensitivity cardiac troponin T values

\begin{tabular}{lcccc}
\hline \multicolumn{1}{c}{ hs-cTnT Roche } & univariate $(95 \% \mathrm{Cl})$ & p-value & multivariate $(95 \% \mathrm{Cl})$ & $\mathrm{p}$-value \\
\hline gender & $8.14(-8.97-25.3)$ & 0.35 & & $0.77(0.93-1.44)$ \\
age & $1.38(0.88-1.87)$ & $<0.001$ & 0.26 \\
history of CAD & $19.08(2.34-35.81)$ & 0.025 & $1.178(-18.37-20.73)$ & 0.91 \\
history of hypertension & $24.09(7.72-40.46)$ & 0.004 & $-0.003(-20.27-20.26)$ & 1.0 \\
positive family history & $26.88(8.76-45.00)$ & 0.004 & $26.57(8.34-44.80)$ & 0.004 \\
eGFR & $-1.00(-1.31-0.69)$ & $<0.001$ & $-0.65(-1.04-0.27)$ & 0.001 \\
admission time & $2.50(0.31-4.96)$ & 0.047 & $2.59(0.08-5.10)$ & 0.04 \\
\hline
\end{tabular}


Supplemental table 4. Early-morning and evening absolute changes of high-sensitivity cardiac troponin $\mathrm{T}$ and I according to final diagnosis

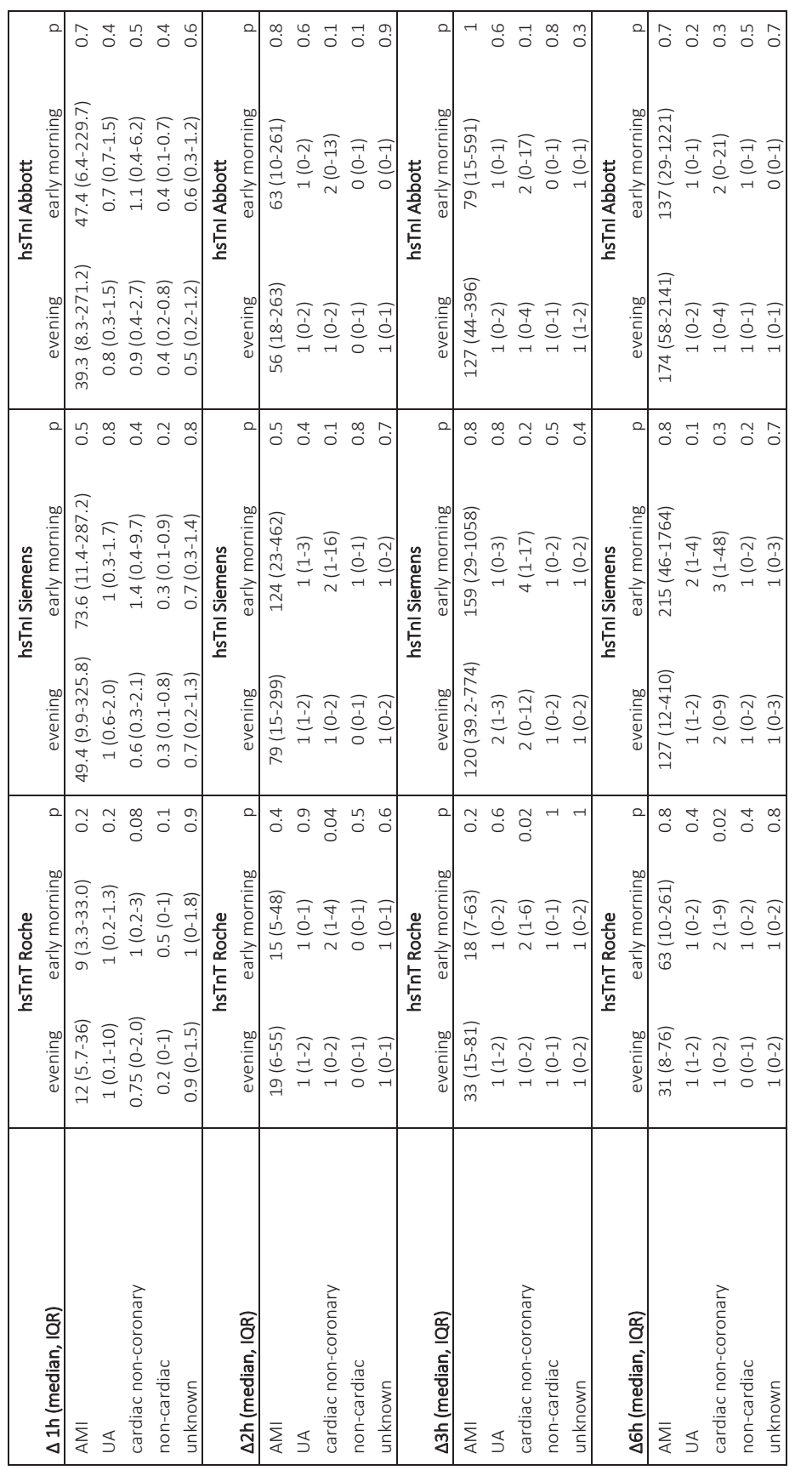


Supplemental table 5. Diagnostic accuracy of absolute changes in high-sensitivity cardiac troponin T and I in a) earlymorning and evening presenters and in b) day and night presenters.

a)

\begin{tabular}{lccr}
\hline hs-cTnT Roche & early morning & evening & p-value \\
\hline$\Delta 1 \mathrm{~h}$ & $0.90(0.88-0.92)$ & $0.95(0.93-0.97)$ & 0.01 \\
$\Delta 2 \mathrm{~h}$ & $0.92(0.90-0.93)$ & $0.95(0.92-0.97)$ & 0.8 \\
$\Delta 3 \mathrm{~h}$ & $0.96(0.94-0.97)$ & $0.97(0.94-0.99)$ & 0.5 \\
$\Delta 6 \mathrm{~h}$ & $0.94(0.91-0.96)$ & $0.97(0.92-0.99)$ & 0.3 \\
\hline hs-cTnl Siemens & early morning & evening & p-value \\
\hline$\Delta 1 \mathrm{~h}$ & $0.93(0.91-0.94)$ & $0.95(0.92-0.97)$ & 0.4 \\
$\Delta 2 \mathrm{~h}$ & $0.93(0.92-0.95)$ & $0.96(0.94-0.98)$ & 0.2 \\
$\Delta 3 \mathrm{~h}$ & $0.93(0.91-0.95)$ & $0.97(0.93-0.99)$ & 0.2 \\
$\Delta 6 \mathrm{~h}$ & $0.92(0.88-0.95)$ & $0.97(0.91-0.99)$ & 0.2 \\
\hline hs-cTnl Abbott & early morning & evening & $\mathrm{p}$-value \\
\hline$\Delta 1 \mathrm{~h}$ & $0.91(0.90-0.94)$ & $0.94(0.92-0.96)$ & 0.2 \\
$\Delta 2 \mathrm{~h}$ & $0.93(0.92-0.95)$ & $0.95(0.92-0.97)$ & 0.5 \\
$\Delta 3 \mathrm{~h}$ & $0.93(0.90-0.95)$ & $0.97(0.92-0.99)$ & 0.2 \\
$\Delta 6 \mathrm{~h}$ & $0.94(0.91-0.97)$ & $0.99(0.94-1.00)$ & 0.1 \\
\hline
\end{tabular}

b)

\begin{tabular}{lccr}
\hline hs-cTnT Roche & day (8 AM to 8 PM) & night (8PM to 8AM) & p-value \\
\hline Oh & $0.93(0.92-0.94)$ & $0.93(0.87-0.96)$ & 1 \\
$1 \mathrm{~h}$ & $0.95(0.94-0.96)$ & $0.94(0.89-0.97)$ & 0.8 \\
$\Delta 1 \mathrm{~h}$ & $0.92(0.90-0.93)$ & $0.92(0.87-0.96)$ & 0.9 \\
$\Delta 2 \mathrm{~h}$ & $0.93(0.91-0.94)$ & $0.88(0.81-0.93)$ & 0.3 \\
$\Delta 3 \mathrm{~h}$ & $0.96(0.94-0.97)$ & $0.98(0.92-1.00)$ & 0.5 \\
$\Delta 6 \mathrm{~h}$ & $0.94(0.91-0.96)$ & $0.98(0.91-1.00)$ & 0.2 \\
\hline hs-cTnl Siemens & day (8 AM to 8 PM) & night (8PM to 8AM) & p-value \\
\hline Oh & $0.92(0.90-0.93)$ & $0.96(0.92-0.99)$ & 0.2 \\
$1 \mathrm{~h}$ & $0.94(0.93-0.95)$ & $0.97(0.92-0.99)$ & 0.3 \\
$\Delta 1 \mathrm{~h}$ & $0.93(0.92-0.95)$ & $0.94(0.88-0.98)$ & 0.9 \\
$\Delta 2 \mathrm{~h}$ & $0.94(0.93-0.96)$ & $0.94(0.87-0.97)$ & 0.9 \\
$\Delta 3 \mathrm{~h}$ & $0.94(0.92-0.96)$ & $0.95(0.87-0.99)$ & 0.9 \\
$\Delta 6 \mathrm{~h}$ & $0.93(0.90-0.95)$ & $0.93(0.83-0.98)$ & 1 \\
\hline hs-cTnl Abbott & day (8 AM to 8 PM) & night (8PM to 8AM) & 0.9 \\
\hline Oh & $0.93(0.92-0.94)$ & $0.94(0.89-0.97)$ & 0.8 \\
$1 \mathrm{~h}$ & $0.94(0.93-0.95)$ & $0.96(0.91-0.99)$ & 0.9 \\
$\Delta 1 \mathrm{~h}$ & $0.93(0.91-0.94)$ & $0.93(0.87-0.97)$ & 0.8 \\
$\Delta 2 \mathrm{~h}$ & $0.94(0.92-0.95)$ & $0.93(0.86-0.97)$ & 0.2 \\
$\Delta 3 \mathrm{~h}$ & $0.94(0.92-0.96)$ & $0.74(0.60-0.85)$ & 0.8 \\
$\Delta 6 \mathrm{~h}$ & $0.96(0.93-0.98)$ & $0.94(0.83-0.99)$ &
\end{tabular}


Supplemental table 6. Prognostic accuracy of high-sensitivity cardiac troponin $\mathrm{T}$ in early-morning and evening presenters for the prediction of all-cause mortality within one year.

\begin{tabular}{llll}
\hline & Early-morning & Evening & \\
& $11.00 \mathrm{PM}-2.00 \mathrm{PM}$ & $2.00 \mathrm{PM}-11.00 \mathrm{PM}$ & \\
\hline AUC $(95 \% \mathrm{Cl})$ & AUC $(95 \% \mathrm{Cl})$ & 0.22 \\
All patients & $0.74(0.71-0.77)$ & $0.82(0.81-0.84)$ & 0.65 \\
Unstable angina & $0.57(0.48-0.66)$ & $0.66(0.37-0.62)$ & 0.28 \\
Cardiac non-coronary & $0.49(0.37-0.62)$ & $0.74(0.67-0.80)$ & 0.90 \\
Non-cardiac & $0.81(0.72-0.88)$ & $0.79(0.74-0.84)$ & 0.53 \\
Unknown & $0.78(0.73-0.82)$ & $0.85(0.83-0.87)$ & \\
\hline
\end{tabular}


Supplemental Table 7. Sensitivity, Specificity, Positive Predictive Value, Negative Predictive Value of baseline values, $1 \mathrm{~h}$ values and changes in hs-cTnT and hs-cTnl in early-morning and evening presenters.

\begin{tabular}{|c|c|c|c|c|}
\hline & $\begin{array}{l}\text { Early-morning } \\
11.00 \text { PM- } \\
2.00 \text { PM }\end{array}$ & $\begin{array}{l}\text { Evening } \\
2.00 \text { PM- } \\
11.00 \mathrm{PM}\end{array}$ & $\begin{array}{l}\text { Early-morning } \\
11.00 \mathrm{PM}- \\
2.00 \mathrm{PM}\end{array}$ & $\begin{array}{l}\text { Evening } \\
2.00 \text { PM- } \\
11.00 \text { PM }\end{array}$ \\
\hline & Sensitivity $(95 \% \mathrm{Cl})$ & Sensitivity $(95 \% \mathrm{Cl})$ & Specificity $(95 \% \mathrm{Cl})$ & Specificity $(95 \% \mathrm{Cl})$ \\
\hline \multicolumn{5}{|c|}{ hs-cTnT Roche } \\
\hline Oh & 99.4 (97.9-99.9) & $100(97.0-100)$ & $28.3(26.1-30.5)$ & $35.0(31.2-39.0)$ \\
\hline $1 \mathrm{~h}$ & 98.9 (96.9-99.8) & $100(96.5-100)$ & $27.8(25.4-30.2)$ & $35.6(31.3-40.1)$ \\
\hline$\Delta 1 \mathrm{~h}$ & $78.2(72.8-82.9)$ & $86.4(78.3-92.4)$ & $93.1(91.6-94.4)$ & $94.1(91.6-96.1)$ \\
\hline $0 h+\Delta 1 h$ & $99.7(98.4-100.0)$ & $100(97.0-100)$ & $65.9(63.3-68.3)$ & $69.6(65.4-73.6)$ \\
\hline \multicolumn{5}{|c|}{ hs-cTn/ Siemens } \\
\hline $\mathrm{Oh}$ & 99.1 (96.7-99.9) & $100(94.9-100)$ & $14.7(12.7-17.0)$ & $15.7(12.4-19.5)$ \\
\hline $1 \mathrm{~h}$ & 98.9 (95.9-99.9) & $100(94.1-100)$ & $12.6(10.5-14.9)$ & $13.1(9.7-17.0)$ \\
\hline$\Delta 1 \mathrm{~h}$ & $91.3(86.0-95.0)$ & $86.9(75.8-94.2)$ & $81.3(78.6-83.8)$ & 86.5 (82.4-89.9) \\
\hline $0 h+\Delta 1 h$ & $98.1(95.3-99.5)$ & $100(94.9-100)$ & $54.1(50.9-57.2)$ & $59.0(53.9-64.0)$ \\
\hline \multicolumn{5}{|c|}{ hs-cTnl Abbott } \\
\hline Oh & $100(98.8-100)$ & $100(96.6-100)$ & $19.8(17.7-22.0)$ & $19.0(15.6-22.7)$ \\
\hline $1 \mathrm{~h}$ & $99.6(97.8-100)$ & $100(96.1-100)$ & $18.5(16.2-20.9)$ & $19.0(15.2-23.2)$ \\
\hline$\Delta 1 \mathrm{~h}$ & $85.2(80.1-89.5)$ & $91.1(83.2-96.1)$ & $87.0(84.8-88.9)$ & $88.8(85.2-91.8)$ \\
\hline $0 h+\Delta 1 h$ & 97.4 (94.9-98.9) & $100(96.6-100)$ & $57.5(54.6-60.3)$ & $59.0(54.1-63.8)$ \\
\hline & PPV $(95 \% \mathrm{Cl})$ & PPV $(95 \% \mathrm{Cl})$ & NPV $(95 \% \mathrm{Cl})$ & NPV $(95 \% \mathrm{Cl})$ \\
\hline \multicolumn{5}{|c|}{ hs-cTnT Roche } \\
\hline Oh & $22.8(20.7-25.0)$ & $23.8(20.2-27.8)$ & $99.6(98.5-100.0)$ & $100(98.3-100)$ \\
\hline $1 \mathrm{~h}$ & $22.1(19.8-24.5)$ & $25.2(21.1-29.7)$ & 99.2 (97.7-99.8) & $100(97.8-100)$ \\
\hline$\Delta 1 \mathrm{~h}$ & $70.0(64.6-75.1)$ & $76.1(67.3-83.5)$ & $95.4(94.1-96.5)$ & $97.0(95.0-98.3)$ \\
\hline $0 h+\Delta 1 h$ & $41.4(38.1-44.9)$ & $43.8(37.8-50.0)$ & $99.9(99.4-100)$ & $100(99.0-100)$ \\
\hline \multicolumn{5}{|c|}{ hs-cTn/ Siemens } \\
\hline Oh & $18.5(16.3-20.9)$ & $16.3(13.0-20.2)$ & $98.8(95.7-99.9)$ & $100(94.6-100)$ \\
\hline $1 \mathrm{~h}$ & $17.4(15.1-20.0)$ & $16.6(13.0-20.8)$ & $98.3(94.1-99.8)$ & $100(92.3-100)$ \\
\hline$\Delta 1 \mathrm{~h}$ & $47.7(42.2-53.3)$ & $53.0(42.8-63.1)$ & $98.0(96.8-98.9)$ & $97.4(95.0-98.9)$ \\
\hline $0 h+\Delta 1 h$ & $31.6(28.1-35.3)$ & $31.1(25.1-37.6)$ & 99.3 (98.1-99.8) & $100(98.4-100)$ \\
\hline \multicolumn{5}{|c|}{ hs-cTnl Abbott } \\
\hline Oh & $21.8(19.7-24.1)$ & $21.1(17.6-24.9)$ & 100 (98.7-100) & $100(96.2-100)$ \\
\hline $1 \mathrm{~h}$ & $21.2(18.9-23.7)$ & $22.8(18.8-27.2)$ & $99.5(97.3-100)$ & $100(95.1-100)$ \\
\hline$\Delta 1 \mathrm{~h}$ & $58.9(53.5-64.2)$ & $66.1(57.1-74.4)$ & $96.4(95.1-97.5)$ & 97.7 (95.4-99.0) \\
\hline $0 h+\Delta 1 h$ & $36.9(33.6-40.3)$ & $38.8(33.0-44.8)$ & $98.9(97.8-99.5)$ & $100(98.5-100)$ \\
\hline
\end{tabular}




\section{Supplemental References}

1. Reichlin T, Hochholzer W, Bassetti S, Steuer S, Stelzig C, Hartwiger S, et al. Early diagnosis of myocardial infarction with sensitive cardiac troponin assays. N Engl J Med 2009;361:858-67.

2. Haaf P, Reichlin T, Twerenbold R, Hoeller R, Rubini Gimenez M, Zellweger $C$, et al. Risk stratification in patients with acute chest pain using three high-sensitivity cardiac troponin assays. Eur Heart J 2014;35:365-75.

3. Reichlin T, Hochholzer W, Stelzig C, Laule K, Freidank H, Morgenthaler NG, et al. Incremental value of copeptin for rapid rule out of acute myocardial infarction. J Am Coll Cardiol 2009;54:60-8.

4. Reiter M, Twerenbold R, Reichlin T, Benz B, Haaf P, Meissner J, et al. Early diagnosis of acute myocardial infarction in patients with pre-existing coronary artery disease using more sensitive cardiac troponin assays. Eur Heart J 2012;33:988-97.

5. Rubini Gimenez M, Twerenbold R, Reichlin T, Wildi K, Haaf P, Schaefer M, et al. Direct comparison of highsensitivity-cardiac troponin I vs. T for the early diagnosis of acute myocardial infarction. Eur Heart J 2014;35:230311.

6. Hamm CW, Bassand JP, Agewall S, Bax J, Boersma E, Bueno H, et al. ESC Guidelines for the management of acute coronary syndromes in patients presenting without persistent ST-segment elevation: The Task Force for the management of acute coronary syndromes (ACS) in patients presenting without persistent ST-segment elevation of the European Society of Cardiology (ESC). Eur Heart J 2011;32:2999-3054.

7. Amsterdam EA, Wenger NK, Brindis RG, Casey DE, Jr., Ganiats TG, Holmes DR, Jr., et al. 2014 AHA/ACC Guideline for the Management of Patients with Non-ST-Elevation Acute Coronary Syndromes: a report of the American College of Cardiology/American Heart Association Task Force on Practice Guidelines. J Am Coll Cardiol 2014;64:e139-228.

8. Keller T, Zeller T, Peetz D, Tzikas S, Roth A, Czyz E, et al. Sensitive troponin I assay in early diagnosis of acute myocardial infarction. N Engl J Med 2009;361:868-77.

9. Thygesen K, Alpert JS, Jaffe AS, Simoons ML, Chaitman BR, White HD, Writing Group on the Joint EAAWTFftUDoMI. Third universal definition of myocardial infarction. Eur Heart J 2012;33:2551-67.

10. Giannitsis E, Kurz K, Hallermayer K, Jarausch J, Jaffe AS, Katus HA. Analytical validation of a high-sensitivity cardiac troponin T assay. Clin Chem 2010;56:254-61.

11. International Diabetes Federation Guideline Development G. Global guideline for type 2 diabetes. Diabetes Res Clin Pract 2014;104:1-52.

12. Inker LA, Schmid CH, Tighiouart H, Eckfeldt JH, Feldman HI, Greene T, et al. Estimating glomerular filtration rate from serum creatinine and cystatin C. N Engl J Med 2012;367:20-9. 


\section{Twenty-four-hour biological variation}

\section{profiles of cardiac troponin I in individuals}

\section{with or without chronic kidney disease}

Noreen van der Linden, Judith Hilderink, Tom Cornelis. Dorien Kimenai, Lieke Klinkenberg, William van Doorn, Elisabeth Litjens, Jeroen van Suijlen, Luc van Loon, Otto Bekers, Jeroen Kooman, Steven Meex - Clin Chem. 2017 Oct;63(10):1655-1656 


\section{Abstract}

\section{Background}

Correct interpretation of serial cardiac troponin measurements is of profound importance for the diagnosis of acute myocardial infarction, especially in populations with a high prevalence of persistently elevated cardiac troponin levels. To gain more insight in physiological cardiac troponin I fluctuations, we performed a biological variation study in which we examined and compared the hour-to-hour biological variation in cardiac troponin I over 24-hours in subjects with and subjects without chronic kidney disease.

\section{Methods}

During $24 \mathrm{~h}$, hourly blood samples were collected from 20 subjects with and 24 subjects without chronic kidney disease. Cardiac troponin I was determined in duplicate. Analytical variation (CVA), withinsubject biological variation (CVI) and between-subject biological variation (CVG) were calculated for 1hour, 3-hour and 6-hour intervals in both groups.

\section{Results}

Baseline cardiac troponin I levels were higher in subjects with chronic kidney disease compared to those without (6.8 [3.5-9.2] ng/L vs. 4.7 [2.8-6.9] ng/L; p=0.09). The within-day variation showed no diurnal pattern. Variation components remained constant for all time-intervals. Whereas $\mathrm{CV}_{\mathrm{G}}(49-95 \%)$ and $\mathrm{CVI}$ (8-9\%) were not significantly different between both groups, CVA was significantly lower in subjects with chronic kidney disease (6\% vs. $10 \%)$.

\section{Conclusions}

Cardiac troponin I concentrations fluctuate at random during the day. Except from the analytical variation, variation components were of the same magnitude in subjects with or without CKD. Our findings imply that the variation in cardiac troponin I levels is not affected by the presence of chronic kidney disease. Interpretation of serial high-sensitivity troponin (hs-cTn) measurements for the diagnosis of acute myocardial infarction (AMI) assumes random fluctuation of hs-cTn around an individual's homeostatic set-point. The aim of this study was to challenge this diagnostic concept. 


\section{Introduction}

The interpretation of cardiac troponin concentrations at presentation and their dynamics over time is a key aspect in the diagnostic workup of acute myocardial infarction in the absence of characteristic ECG-abnormalities ${ }^{1,2}$. Whereas subjects with very low or high cardiac troponin concentrations at presentation can immediately be ruled-out or ruled-in respectively, serial measurements are particularly indicated in patients with moderately elevated cardiac troponin concentrations ${ }^{3-6}$. A majority of these patients does not have an acute myocardial infarction, but shows persistently elevated cardiac troponin concentrations which are associated with the presence of underlying (subclinical) disease like diabetes, coronary artery disease, hypertension and chronic kidney disease $(C K D)^{7-9}$. Especially in these patients it is of profound importance to distinguish pathology associated changes from physiological fluctuation in serial cardiac troponin measurements.

Biological variation studies provide insight in the magnitude of physiological variation. On the basis is the assumption that the biomarker concentrations fluctuate around an individual set point, and that the totally observed variation can be subdivided in a between-subject, a within-subject and an analytical component ${ }^{10}$. These individual components facilitate the estimation of the reference change value (RCV), the required threshold for a change between serial measurements to be significantly different from a physiological variation. Additionally, it is possible to calculate the index of individuality (II), which represents the variation between the individual set points ${ }^{11-13}$.

During the last decade, the duration of the diagnostic workup for acute myocardial infarction shortened, which was reflected in the recent introduction of the $0 \mathrm{~h} / 1 \mathrm{~h}$-algorithm ${ }^{1,14,15}$. Since most biological variation studies focus on long(er)-term variation in cardiac troponin I $(\mathrm{cTnl})^{16-19}$, less is known about the hour-to-hour variation. Furthermore, all previous reported studies were limited to a certain time interval and it is therefore not clear whether the derived values apply to both day and night, or may be affected by a diurnal rhythm ${ }^{20}$. The present biological variation study was the first to examine and compare the hour-to-hour biological variation in cTnl over 24-hours in subjects without and subjects with CKD. 


\section{Methods}

This study was carried out according to the principles of the Declaration of Helsinki, approved by the Institutional Review Board and Ethics Committee of Maastricht University Medical Center, and registered at clinicaltrial.gov (NCT02091427 and NCT02210897). All participants provided written informed consent. The current study meets the critical appraisal checklist criteria for studies of biological variation as proposed by Bartlett et al. ${ }^{21}$.

\section{Study population}

The study population consisted of 44 adult subjects, 24 subjects ( 19 males and 5 females) without CKD and 20 subjects (14 males and 6 females) with CKD. The subjects without CKD were previously described in more detail20. Subjects with CKD stage 3 or higher (estimated glomerular filtration rate $\left.(\mathrm{eGFR})<59 \mathrm{~mL} / \mathrm{min} / 1.73 \mathrm{~m}^{2}\right)^{22}$ were included at the nephrology outpatient department of the Maastricht University Medical Center (MUMC), and suffered from various types of kidney disease (glomerular $(n=7)$, tubulointerstitial $(n=4)$, vascular $(n=8)$ and congenital kidney disease $(n=1))$. Exclusion criteria were: history of acute myocardial infarction less than 12 months prior to exclusion, (current) dialysis regimen, active cardiac disease (angina pectoris, cardiomyopathy and myocarditis) and anemia ( $\mathrm{Hb}<$ $10.5 \mathrm{~g} / \mathrm{dL}$ ). To monitor whether the degree of renal impairment was stable in CKD subjects, we examined kidney function (estimated glomerular filtration rate (eGFR)) one week and one month after the initial test day.

\section{Study design}

After an overnight fast, subjects arrived at 8 a.m. at the laboratory by car or public transport. For 25h, from 8.30 A.M. till 9.30 A.M. the next day, subjects were restricted to the laboratory environment, and samples were collected every hour from an antecubital venous catheter. Extension lines for blood sampling were used to prevent disturbance of participants' sleep during the night. Meals were consumed at 8.30 A.M., 12.30 P.M. and 6.00 P.M. (breakfast, lunch and dinner, respectively). Subjects went to bed at 11.30 P.M., lights were off between 
11.35 P.M. and 7.00 A.M., and they slept in a supine position. Participants were asked to refrain from exhaustive physical activities and exercise training, two days before the test day.

\section{Laboratory measurements}

Blood samples were collected in ethylenediaminetetraacetic acid containing (EDTA) tubes. Samples were centrifuged and the plasma was stored at $-80^{\circ} \mathrm{C}$ until analysis. cTnl was measured with the STAT high-sensitivity troponin I assay (Architect i2000 SR, Abbott Diagnostics). According to the manufacturer, the LoD is $1.1-1.9 \mathrm{ng} / \mathrm{L}$, and 99th percentile of this assay is $26.2 \mathrm{ng} / \mathrm{L}$ with a corresponding coefficient of variation of $<5 \%$. To enable the determination of analytical variation, all samples were measured in duplicate. Creatinine and cystatin C were both measured on the COBAS 8000 analyzer. The eGFR was calculated according to the Chronic Kidney Disease Epidemiology Collaboration (CKD-EPI) formula based on creatinine and cystatin $\mathrm{C}^{23}$.

\section{Statistical analysis}

For the comparison between the baseline characteristics of both groups, continuous variables were compared with the Mann-Whitney $U$ test, and categorical variables were compared by use of the Pearson $\chi^{2}$ test. Data are expressed as median and interquartile range (median [IQR]) for non-normally distributed continuous variables and as numbers and percentages $(n(\%))$ for categorical variables.

For the 24-hour analyses, we used data obtained between 10.30 AM (first day) till 9.30 (AM) (second day). As suggested by Fraser and Harris ${ }^{24}$, we tested for homogeneity in the analytical- and within-subject biological variances using the method described by Cochran. Finally, the criteria of Reed were used to identify between-subject outliers ${ }^{10}$.

Two-fold nested ANOVA was used to calculate the coefficients of variation on a betweensubject $\left(C V_{G}\right)$, within-subject $\left(C V_{I}\right)$ and an analytical $\left(C V_{A}\right)$ level, including 95\% confidence intervals ${ }^{25,26}$. We calculated the reference change value (RCV) using the formula: $R C V=Z \cdot V\left(2 \cdot\left(C V_{1}^{2}+C V_{A}^{2}\right)\right)$. In the RCV formula, $Z$ represents the number of standard deviations appropriate for the desired level of statistical significance for a bidirectional change ${ }^{10}$. For 
the RCV calculations in this study a Z-score of 1.96 was used. The index of individuality (II) was calculated using the formula: $\|=V\left(C V_{1}{ }^{2}+C V_{A}{ }^{2}\right) / C V_{G}{ }^{13,27}$. All hypothesis testing was twotailed, and values of $\mathrm{P}<0.05$ were considered statistically significant. Statistical analyses were performed with SPSS for Windows 23.0 (IBM Corp., Armonk, NY, USA).

\section{Results}

A total of 44 subjects, 20 with and 24 without CKD disease, participated in this biological variation study. We plotted the range in observed cTnl concentrations over $24 \mathrm{~h}$ for all individual subjects in Figure 1.

A

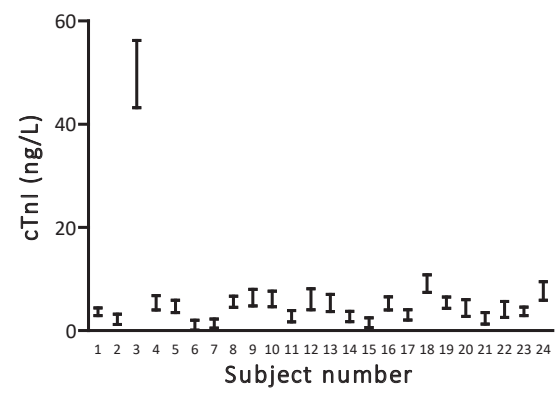

B

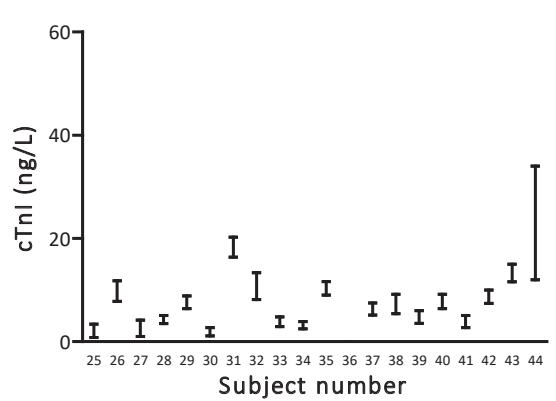

Figure 1. Subject specific concentrations over $24 \mathrm{~h}$ with hourly sampling. Absolute ranges ( $\mathrm{min}-\mathrm{max}$ ) of cTnl concentrations over $24 \mathrm{~h}$ in subjects without CKD (panel A) and with CKD (panel B).

Complete data sets were available for 40 subjects (subjects 1, 8, 15 and 23 had missing data). In addition, one subject had no measurable levels of cTnl (subject 36), and another subject became sick during the test day (subject 20). Two subjects (subjects 3 and 44) were identified as outliers and were therefore excluded from further analyses. A total of 36 subjects, 18 subjects with and 18 subjects without CKD, were included in the final data analyses. Figure 2 shows the in- and exclusion of subjects, including baseline characteristics. In subjects with CKD the eGFR remained stable for at least one month after the initial test day (data not shown). 
Baseline cTnl levels were higher in subjects with CKD compared to those without CKD (6.8 [3.5-9.2] $\mathrm{ng} / \mathrm{L}$ vs. 4.7 [2.8-6.9] $\mathrm{ng} / \mathrm{L} ; \mathrm{p}=0.09)$. The within-day variation in these concentrations showed no diurnal pattern, and biological variation seemed to be constant over 24h in both groups (Figure 3, Supplemental Figure 1) (individual curves of subjects without CKD have been previously published ${ }^{20}$; chapter 3 of this thesis).

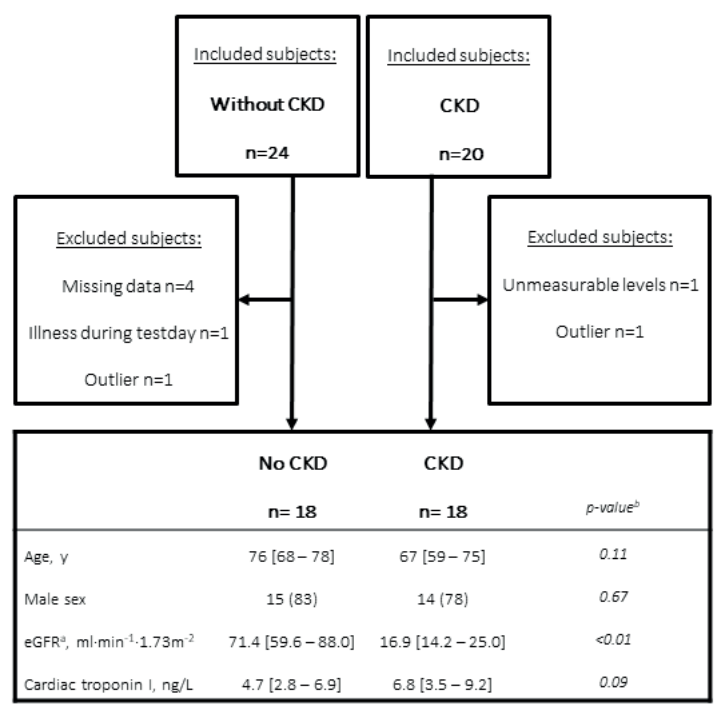

Figure 2. Flowchart of the study, including baseline characteristics. ${ }^{a}$ eGFR, estimated glomerular filtration rate according to the CKD-EPI Creatinine-Cystatin C equitation ${ }^{23}$; ${ }^{b}$ comparison between subjects with or without CKD.

Consecutively, we calculated the variation components of 1-hour, 3-hour and 6-hour intervals during the day, which are presented in Table 1. We detected no significant differences in the components of variation for the different time intervals. When the variation components where compared between subjects without and subjects with CKD, $\mathrm{CV}_{\mathrm{G}}$ and $\mathrm{CV}$, were not significantly different. In contrast, we observed significant differences in $\mathrm{CV}_{\mathrm{A}}$ between both groups: $\mathrm{CV}_{\mathrm{A}}$ was significantly lower in subjects with CKD (the group with 
A

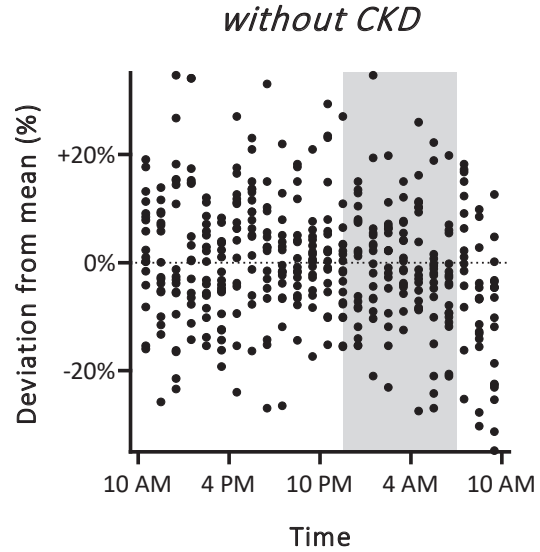

B

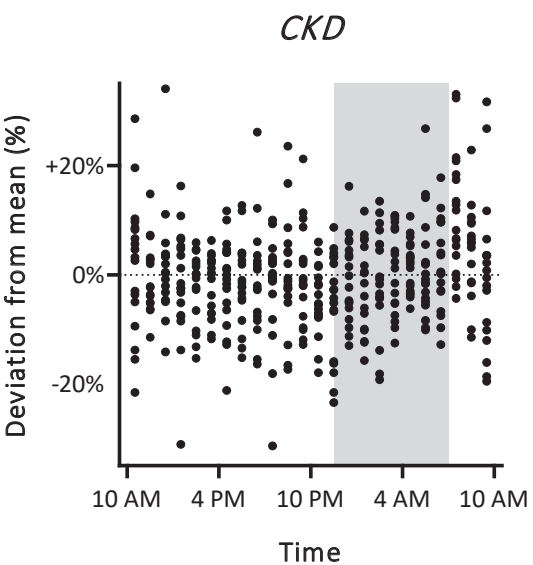

Figure 3. No diurnal pattern in cTnl variation in subjects with or without CKD. We calculated the deviation (in \%) from the mean concentration (over 24h) of each individual on every time point in subjects with (panel A) or without CKD (panel B). This variation is random and is not subject to a circadian pattern.

Table 1. Hour-to-hour biological variation in subjects with or without chronic kidney disease

\begin{tabular}{|c|c|c|c|c|c|c|}
\hline & \multicolumn{2}{|c|}{ 1-hour } & \multicolumn{2}{|c|}{ 3-hourc } & \multicolumn{2}{|c|}{ 6-hour ${ }^{d}$} \\
\hline & No CKD & CKD & No CKD & CKD & No CKD & CKD \\
\hline & $n=18$ & $n=18$ & $\mathrm{n}=18$ & $\mathrm{n}=18$ & $n=18$ & $\mathrm{n}=18$ \\
\hline \multicolumn{7}{|c|}{ Variance components } \\
\hline \multirow[t]{2}{*}{$\mathrm{CV}_{\mathrm{G}}$} & 49.4 & 62.4 & 48.0 & 61.6 & 48.6 & 61.6 \\
\hline & $(37.1-74.2)$ & $(46.8-93.5)$ & $(35.9-71.1)$ & $(46.2-92.4)$ & $(36.2-73.1)$ & $(46.1-92.5)$ \\
\hline \multirow[t]{2}{*}{$\mathrm{CV}_{\mathrm{I}}$} & 8.6 & 7.7 & 9.4 & 8.7 & 9.2 & 8.9 \\
\hline & $(7.6-9.7)$ & $(7.1-8.4)$ & $(7.6-11.5)$ & $(7.5-10.2)$ & $(6.2-12.4)$ & $(7.1-11.3)$ \\
\hline \multirow[t]{2}{*}{$\mathrm{CV}_{\mathrm{A}^{\mathrm{a}}}$} & 10.0 & 5.6 & 9.9 & 5.6 & 10.1 & 5.9 \\
\hline & $(9.4-10.8)$ & $(5.2-6.0)$ & $(8.8-11.2)$ & $(5.0-6.3)$ & $(8.7-12.1)$ & $(5.0-7.0)$ \\
\hline \multicolumn{7}{|l|}{$R C V^{p}$} \\
\hline Normal & 36.7 & 26.4 & 37.9 & 28.6 & 37.5 & 29.5 \\
\hline Log-normal & $-30.6 ; 44.0$ & $-23.16 ; 30.15$ & $-31.4 ; 45.8$ & $-24.8 ; 33.0$ & $-31.4 ; 45.7$ & $-25.5 ; 34.2$ \\
\hline \multicolumn{7}{|c|}{ Index of individuality } \\
\hline & 0.27 & 0.15 & 0.28 & 0.17 & 0.28 & 0.17 \\
\hline
\end{tabular}

Values are \% $(95 \% \mathrm{Cl}) ; \mathrm{Cl}=$ confidence interval; $\mathrm{CV}_{\mathrm{A}}=$ analytical coefficient of variation; $\mathrm{CV}_{\mathrm{G}}=$ between-subject coefficient of variation; $\mathrm{CV}_{1}=$ within-subject biological coefficient of variation; $\mathrm{RCV}=$ reference change value ${ }^{a}$ on the basis of duplicate measurements; ${ }^{b}$ on the basis of a Z-score of 1.96; ${ }^{c}$ 3-hour intervals: 10:30 AM-01:30 PM, 01:30 PM-04:30 PM, 04:30 PM-07:30 PM, 07:30 PM-10:30 PM, 01:30 AM-4:30 AM and 4:30 AM-7:30 AM; ${ }^{d}$ 6-hour intervals: 10:30 AM-04:30 PM, 04:30 PM-10:30 PM and 10:30 PM-04:30 AM 
the higher cTnl concentrations). As a result of the lower $\mathrm{CV}_{\mathrm{A}}$, the RCV is also lower among these subjects. Besides, we observed a trend towards a higher between-subject variation and a lower index of individuality in this group.

\section{Discussion}

This study examined the hour-to-hour biological variation of cTnl, and calculated its variation components over $24 \mathrm{~h}$ in subjects with or without CKD. We report four major findings: First, we observed randomly fluctuating cTnl levels over the day. In the absence of a circadian rhythm, we calculated the 1-hour, 3-hour and 6-hour variation over the day without any additional transformations. The fact that the $\mathrm{CV}_{\text {I }}$ remained constant (8-9\%) during all timeintervals over $24 \mathrm{~h}$, supports our observation of random variation. Second, the CVI was not significantly different in subjects with or without CKD. This seems to be in line with the claim

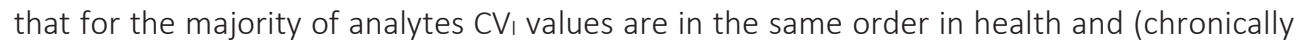
stable) disease $\mathrm{e}^{10,28}$. Third, we identified a substantial amount of variation between individuals $\left(C V_{G}=48-62 \%\right)$. This finding corresponds to that of previous reported studies ${ }^{16-18,29,30}$ and, since the $C_{\text {I }}$ is rather limited, it produces a low index of individuality $(<0.3)$ The observation that the $C_{G}$ is slightly higher in subjects with CKD may be due to a high variety in comorbidities characteristic for this population ${ }^{31}$. Fourth, we showed that the $\mathrm{CV}_{\mathrm{A}}$ is significantly higher in subjects without than in those with CKD (10\% vs. $6 \%$ ). This can be explained by the concentration-dependency of the $\mathrm{CV}_{\mathrm{A}}$. As a consequence of these differences in $\mathrm{CV}_{\mathrm{A}}$, the RCV is slightly lower in the group with the higher cTnl concentrations. From a clinical point of view, the finding of a substantial $\mathrm{CV}_{\mathrm{G}}$ and a low index of individuality emphasizes the wide variety in cardiac troponin concentrations among the population, the limited utility of population-based reference intervals and the importance of the use of deltachange values ${ }^{13,32}$. Nevertheless, it is important to consider that the ongoing progression towards increasingly short time intervals between serial measurements, will come to a stop at the point where the changes between consecutive concentrations are so small that there is no distinction anymore with the physiological variation within a person. This can only be partially overcome by increasing the sensitivity and precision of the assay: In the hypothetical 
situation of a perfect assay $\left(\mathrm{CV}_{\mathrm{A}} \mathrm{0 \%}\right)$ and a $\mathrm{CV}$ । of approximately $8 \%$, the calculated $\mathrm{RCV}$ is $22 \%$. This implies that the RCV for cTnI values in the lower range may drop from $37 \%$ to $22 \%$, but will never be lower than $22 \%$.

Potential limitations of our study merit consideration. First, most subjects included in the final data analyses have relatively low concentrations of cTnl. It is therefore hard to extrapolate our findings to higher concentration ranges. However, when cTnl values at presentation are much higher, serial measurements have limited additional value ${ }^{1,3}$. Second, since the prevalence of comorbidities in both populations is relatively high ${ }^{20}$, we cannot make an unbiased comparison between health and disease. Therefore, we were only able to examine the effect of chronic kidney disease.

In conclusion, with this biological variation study we were the first to examine the hour-tohour biological variation in cTnl over a full time span of $24 \mathrm{~h}$. We showed a random variation over the day. Except from the analytical variation, variation components were of the same magnitude in subjects with or without CKD. Our findings imply that the within-subject biological variation of cardiac troponin I is not affected by the presence of chronic kidney disease. 


\section{References}

1. Roffi M, Patrono C, Collet JP, et al. 2015 ESC Guidelines for the management of acute coronary syndromes in patients presenting without persistent ST-segment elevation: Task Force for the Management of Acute Coronary Syndromes in Patients Presenting without Persistent ST-Segment Elevation of the European Society of Cardiology (ESC). Eur Heart J 2016;37:267-315.

2. Amsterdam EA, Wenger NK, Brindis RG, et al. 2014 AHA/ACC Guideline for the Management of Patients with NonST-Elevation Acute Coronary Syndromes: a report of the American College of Cardiology/American Heart Association Task Force on Practice Guidelines. J Am Coll Cardiol 2014;64:e139-228.

3. Mueller-Hennessen M, Mueller C, Giannitsis E, et al. Serial Sampling of High-Sensitivity Cardiac Troponin T May Not Be Required for Prediction of Acute Myocardial Infarction Diagnosis in Chest Pain Patients with Highly Abnormal Concentrations at Presentation. Clin Chem 2017;63:542-51.

4. Body R, Burrows G, Carley S, et al. High-sensitivity cardiac troponin t concentrations below the limit of detection to exclude acute myocardial infarction: a prospective evaluation. Clin Chem 2015;61:983-9.

5. Sandoval Y, Smith SW, Shah AS, et al. Rapid Rule-Out of Acute Myocardial Injury Using a Single High-Sensitivity Cardiac Troponin I Measurement. Clin Chem 2017;63:369-76.

6. Boeddinghaus J, Reichlin T, Nestelberger T, et al. Early diagnosis of acute myocardial infarction in patients with mild elevations of cardiac troponin. Clin Res Cardiol 2017.

7. Nestelberger T, Wildi K, Boeddinghaus J, et al. Characterization of the observe zone of the ESC 2015 highsensitivity cardiac troponin $0 \mathrm{~h} / 1 \mathrm{~h}$-algorithm for the early diagnosis of acute myocardial infarction. Int J Cardiol 2016;207:238-45.

8. Twerenbold R, Wildi K, Jaeger C, et al. Optimal Cutoff Levels of More Sensitive Cardiac Troponin Assays for the Early Diagnosis of Myocardial Infarction in Patients With Renal Dysfunction. Circulation 2015;131:2041-50.

9. de Lemos JA. Increasingly sensitive assays for cardiac troponins: a review. JAMA 2013;309:2262-9.

10. Fraser C. Biological Variation: From principles to practice. Washington: AACC press; 2001.

11. Braga F, Panteghini M. Generation of data on within-subject biological variation in laboratory medicine: An update. Crit Rev Cl Lab Sci 2016;53:313-25.

12. Fraser CG. Reference change values. Clin Chem Lab Med 2011;50:807-12.

13. Harris EK. Effects of intra- and interindividual variation on the appropriate use of normal ranges. Clin Chem 1974;20:1535-42.

14. Reichlin T, Schindler C, Drexler B, et al. One-hour rule-out and rule-in of acute myocardial infarction using highsensitivity cardiac troponin T. Arch Intern Med 2012;172:1211-8.

15. Rubini Gimenez M, Twerenbold R, Jaeger C, et al. One-hour rule-in and rule-out of acute myocardial infarction using high-sensitivity cardiac troponin I. Am J Med 2015;128:861-70 e4.

16. Wu AH, Akhigbe P, Wians F. Long-term biological variation in cardiac troponin I. Clin Biochem 2012;45:714-6.

17. Wu AH, Shea E, Lu QT, et al. Short- and long-term cardiac troponin I analyte stability in plasma and serum from healthy volunteers by use of an ultrasensitive, single-molecule counting assay. Clin Chem 2009;55:2057-9.

18. Simpson AJ, Potter JM, Koerbin G, et al. Use of observed within-person variation of cardiac troponin in emergency department patients for determination of biological variation and percentage and absolute reference change values. Clin Chem 2014;60:848-54.

19. Apple FS, Collinson PO, Biomarkers ITFoCAoC. Analytical characteristics of high-sensitivity cardiac troponin assays. Clin Chem 2012;58:54-61.

20. Klinkenberg $\amalg$, Wildi K, van der Linden N, et al. Diurnal Rhythm of Cardiac Troponin: Consequences for the Diagnosis of Acute Myocardial Infarction. Clin Chem 2016;62:1602-11.

21. Bartlett WA, Braga F, Carobene A, et al. A checklist for critical appraisal of studies of biological variation. Clin Chem Lab Med 2015;53:879-85.

22. Levey AS, Stevens LA, Coresh J. Conceptual model of CKD: applications and implications. Am J Kidney Dis 2009;53:S4-16. 
23. Inker LA, Schmid CH, Tighiouart $\mathrm{H}$, et al. Estimating glomerular filtration rate from serum creatinine and cystatin C. N Engl J Med 2012;367:20-9.

24. Fraser CG, Harris EK. Generation and application of data on biological variation in clinical chemistry. Crit Rev Clin Lab Sci 1989;27:409-37.

25. Roraas T, Petersen PH, Sandberg S. Confidence Intervals and Power Calculations for Within-Person Biological Variation: Effect of Analytical Imprecision, Number of Replicates, Number of Samples, and Number of Individuals (vol 58, pg 1306, 2012). Clinical Chemistry 2012;58:1724-.

26. Burdick RK GF. Confidence intervals on variance components. New York: Marcel Dekker, Inc; 1992.

27. Petersen PH, Fraser CG, Sandberg S, Goldschmidt H. The index of individuality is often a misinterpreted quantity characteristic. Clin Chem Lab Med 1999;37:655-61.

28. Ricos C, Iglesias N, Garcia-Lario JV, et al. Within-subject biological variation in disease: collated data and clinical consequences. Ann Clin Biochem 2007;44:343-52.

29. Aakre KM, Roraas T, Petersen PH, et al. Weekly and 90-Minute Biological Variations in Cardiac Troponin T and Cardiac Troponin I in Hemodialysis Patients and Healthy Controls. Clinical Chemistry 2014;60:838-47.

30. Mbagaya W, Luvai A, Lopez B. Biological variation of cardiac troponin in stable haemodialysis patients. Ann Clin Biochem 2015;52:562-8.

31. Tonelli M, Wiebe N, Guthrie B, et al. Comorbidity as a driver of adverse outcomes in people with chronic kidney disease. Kidney Int 2015;88:859-66.

32. Harris EK. Statistical aspects of reference values in clinical pathology. Prog Clin Pathol 1981;8:45-66. 


\section{Supplemental information}

\section{Supplemental Figures}
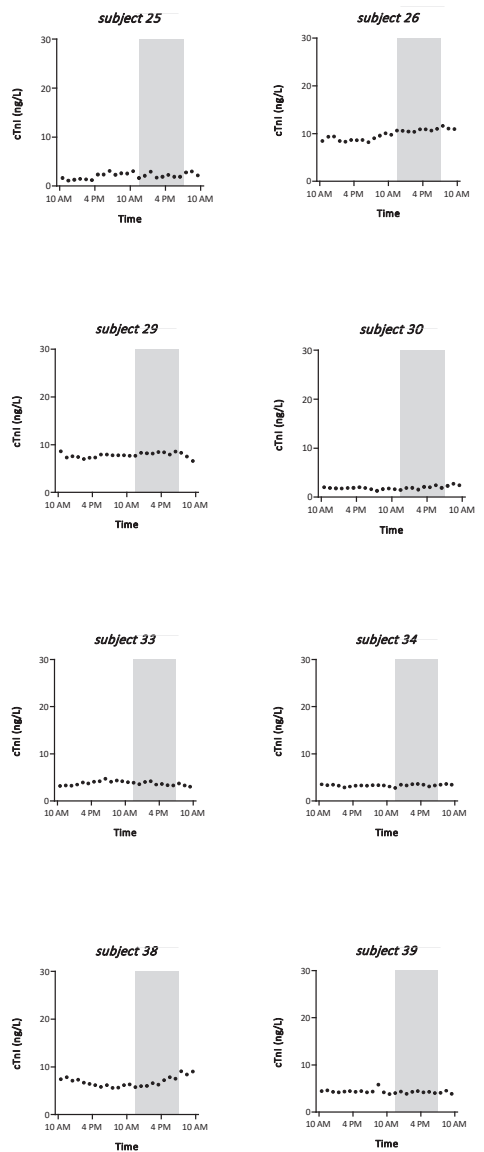

Time

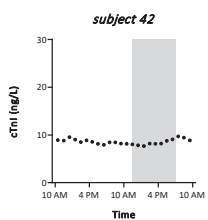

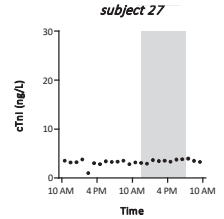
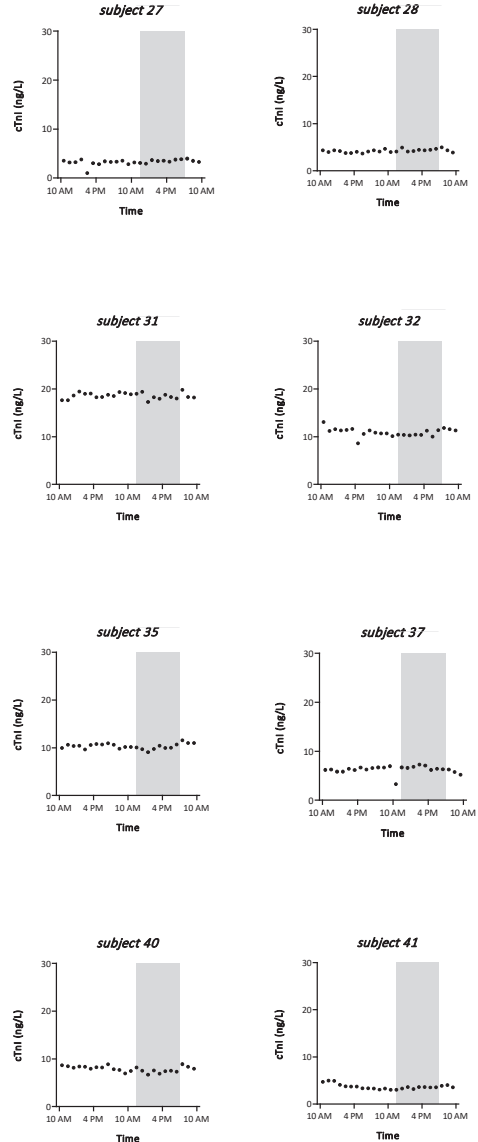

Supplemental Figure 1. Individual diurnal cardiac troponin I profiles. cTnl concentrations over the day in subjects with chronic kidney disease. Depicted are the mean of the duplicate measurements. The numbering is identical to Figure 1. Subjects slept in a supine position between 11:30 P.M. and 7:00 A.M. (shaded area) (curves of non-CKD subjects are shown in chapter 3 ). 
Cardiac troponins: State of the (He)Art 


\section{Origin of cardiac troponin T elevations in}

\section{chronic kidney disease}

Noreen van der Linden, Tom Cornelis, Dorien Kimenai, Lieke Klinkenberg, Judith Hilderink, Sarah Lück, Elisabeth Litjens, Frederqive Peeters, Sander Streng, Tobias Breidthardt, Luc van Loon, Otto Bekers, Jeroen Kooman, Pål Westermark, Christian Mueller, Steven Meex Circulation. 2017 Sep 12,136(11):1073-1075 


\section{Abstract}

\section{Background}

Elevated cardiac troponin concentrations are common among patients with impaired renal function. Their origin is unknown, but may involve the heart, by increased release, or the kidneys, by decreased renal elimination.

\section{Methods}

We employed the diurnal rhythm in troponin T as a model to assess the contribution of impaired renal function to elevated troponin levels in subjects with impaired renal function. We obtained blood samples over 24 hours from subjects with $(n=24)$ and without $(n=20)$ impaired renal function. Diurnal rhythms of cardiac troponin $T$ were analysed by fitting data to a cosine curve. Expected fading of rhythmicity upon a progressively decreasing renal elimination rate was modelled using the cosine curves of subjects without impaired renal function, and compared with the amplitudes in subjects with impaired renal function.

\section{Results}

Baseline cardiac troponin T levels were significantly higher in the group with, than in the group without impaired renal function (median 20.5 [18.0-24.6] vs. 14.1 [10.4-20.6] ng/L, p=0.009). Concentrations of cardiac troponin T over the day fitted a cosinor model in all subjects (range $R^{2} 0.23-0.91$; all $p \leq 0.001$ ). A progressively decreasing renal elimination was mathematically predicted to decrease the amplitude of the diurnal troponin T rhythm. However, the actual amplitudes of the diurnal rhythm in subjects with chronic kidney disease followed a similar pattern, and were not reduced in amplitude compared to subjects without CKD ( $11.4 \pm 0.5$ vs. $12.0 \pm 0.5 \%$; $p=0.53$; mathematically predicted amplitude $8 \%)$. In contrast, the amplitude of cystatin C was significantly faded among subjects with decreased renal function compared with the reference group. $1.7 \pm 0.2 \%$ vs. $3.9 \pm 0.2 \% ; p<0.001$; mathematically predicted amplitude $2 \%)$.

\section{Conclusions}

Whereas a severely decreased elimination of cardiac troponin T would lead to accumulation of cardiac troponin $T$ and fading of the diurnal rhythm, our results suggest that impaired renal elimination itself is not the main cause of elevated cardiac troponin T levels in subjects with chronic kidney disease. 


\section{Introduction}

Plasma concentrations of cardiac troponins, the preferred biomarkers for the diagnosis of acute myocardial infarction ${ }^{1-3}$, are often persistently elevated in patients with chronic kidney disease $(C K D)^{4}$. Epidemiological cohort studies support this clinical observation and show robust associations between a deteriorating glomerular filtration rate and plasma troponin elevations $s^{5-7}$. The origin of elevated cardiac troponin concentrations in these patients is unknown: Is it the heart, by increased release, or the kidneys, by decreased renal elimination ${ }^{7-9}$ ? CKD is strongly associated with an increased incidence of cardiovascular disease ${ }^{10,11}$, supporting increased cardiac vulnerability of these patients. Nevertheless, by some, elevated plasma troponin levels in CKD patients are regarded as the prototype example of troponin elevations without cardiovascular etiology 8,12 . In clinical practice, this equivocal view on troponin elevations in patients with reduced glomerular clearance underlies countless clinical discussions among physicians and may delay rapid initiation of adequate treatment when these patients present with chest pain ${ }^{4,13}$.

In the present study we sought to discriminate between 'increased cardiac release' or 'reduced renal elimination' as the main process underlying this phenomenon. Specifically, we employed the recently demonstrated rhythmic diurnal oscillation pattern of troponin $\mathrm{T}$, characterized by gradually decreasing concentrations throughout daytime and rising concentrations during night-time, as a model to assess the contribution of impaired renal elimination to persistently elevated troponin levels in in patients with $\mathrm{CKD}^{14,15}$. We hypothesized that if decreased renal clearance, and not increased production, is the main driver behind the elevated levels of cardiac troponins in patients with CKD, the subsequent accumulation of cardiac troponins will fade the diurnal rhythm of cardiac troponin $\mathrm{T}$. Therefore, by comparing the amplitude of diurnal oscillations in cardiac troponin T concentrations in subjects with or without CKD, we evaluated the role of reduced renal elimination on elevated basal cardiac troponin $T$ concentrations ${ }^{16}$. 


\section{Methods}

To test the hypothesis that impaired renal elimination is the main driver behind elevated cardiac troponin concentrations, and that the diurnal rhythm in cardiac troponin T levels is affected by the prolonged terminal elimination time in subjects with severely decreased renal function, we performed hourly blood sampling over one full day ( $24 \mathrm{~h}$ ), in 20 reference subjects (estimated glomerular filtration rate (eGFR) above $60 \mathrm{ml} \cdot \mathrm{min}-1 \cdot 1.73 \mathrm{~m}-2$ ) and in 24 subjects with decreased renal function (eGFR below $60 \mathrm{ml} \cdot \mathrm{min}-1 \cdot 1.73 \mathrm{~m}-2)^{17}$. Two consecutive analyses were performed: First, to examine how the rhythm in cardiac troponin T would change due to a decreased elimination rate, we applied a mathematical model, that quantifies the effect of decreased elimination on the resulting diurnal cosine curve, to the circadian rhythm that was observed in the reference subjects ${ }^{15}$. Second, we assessed whether the predicted effect of prolonged elimination time on the troponin amplitude matched with the actual results obtained in subjects with impaired renal function.

The study was carried out according to the principles of the Declaration of Helsinki, approved by the Institutional Review Board and Ethics Committee of Maastricht University Medical Centre, and registered at clinicaltrials.gov (NCT02091427 and NCT02210897). All participants provided written informed consent.

\section{Study participants}

A total of 44 adult subjects, 20 reference subjects (16 males, 4 females, median eGFR 82.8 [68.0-92.7] $\mathrm{ml} \cdot \mathrm{min}^{-1} \cdot 1.73 \mathrm{~m}^{-2}$ ), and 24 subjects with impaired renal function (eGFR below 60 $\left.\mathrm{ml} \cdot \mathrm{min}^{-1} \cdot 1.73 \mathrm{~m}^{-2}\right)(17 \text { males, } 7 \text { females, median eGFR } 18.3 \text { [14.2-32.2] } \mathrm{ml} \cdot \mathrm{min}-1 \cdot 1.73 \mathrm{~m}-2)^{15}$, were consecutively included. Exclusion criteria were: history of acute myocardial infarction less than 12 months prior to exclusion, (current) dialysis regimen, active cardiac disease (angina pectoris, cardiomyopathy and myocarditis) and anemia ( $(\mathrm{Hb}<10.5 \mathrm{~g} / \mathrm{dL})$. To facilitate the detection of a rhythm in cardiac troponin T levels, all included subjects were selected for their elevated basal cardiac troponin T levels. 


\section{Day and night hourly blood sampling}

After an overnight fast, subjects arrived at the laboratory by car or public transport. For 25 h, from 8.30 AM till 9.30 AM the next day, subjects were restricted to the laboratory environment, and samples were collected every hour from an antecubital venous catheter. Extension lines for blood sampling were used to prevent disturbance of participants' sleep during the night. Meals were consumed at 8.30 AM, 12.30 PM and 6.00 PM (breakfast, lunch and dinner, respectively). Subjects went to bed at 11.30 PM, lights were off between 11.35 $\mathrm{PM}$ and $7.00 \mathrm{AM}$, and they slept in a supine position. Participants were asked to refrain from exhaustive physical activities and exercise training, two days before the test day. A schematic overview of the test day is shown in Figure 1.

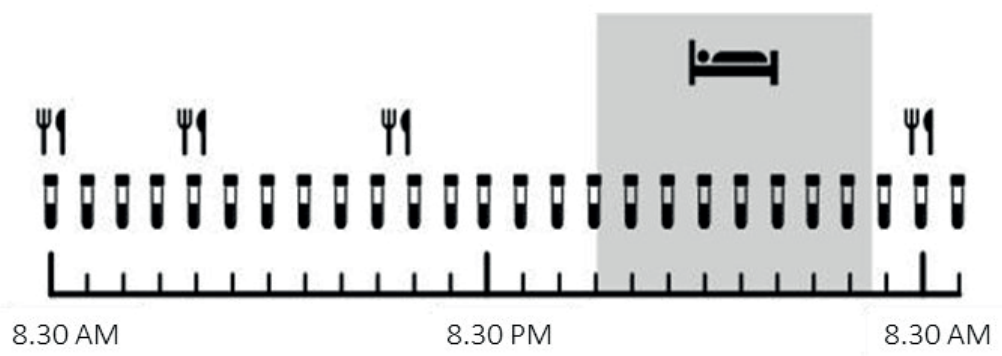

Figure 1. Hourly blood sampling protocol in subjects with or without chronic kidney disease. Between 8.30 AM till 9.30 AM the next day, subjects were restricted to the laboratory environment, and samples were collected every hour. The lights were off between 11.35 PM and 7.00 AM (grey part), and subjects slept in a supine position. Meals breakfast, lunch and dinner - were consumed at 8.30 A.M., 12.30 PM and 6.00 PM.

\section{Laboratory measurements}

Blood samples were collected in ethylenediaminetetraacetic acid containing (EDTA) tubes. Samples were centrifuged and plasma was stored at $-80^{\circ} \mathrm{C}$ until analysis. Cardiac troponin $\mathrm{T}$ was measured with the high-sensitivity cardiac troponin T assay (Roche) on a COBAS 6000 analyser. The 99th percentile among healthy subjects is $14 \mathrm{ng} / \mathrm{L}$, with a $10 \%$ analytical variation at $13 \mathrm{ng} / \mathrm{L}^{18}$. N-terminal prohormone of brain natriuretic peptide (NT-proBNP), creatinine and cystatin $\mathrm{C}$ were also measured on the COBAS analyser (all with assays from Roche). The estimated glomerular filtration rate (eGFR) was calculated according to the 
Chronic Kidney Disease Epidemiology Collaboration formula based on creatinine and cystatin $\mathrm{C}^{19}$.

\section{Statistical analysis}

Data are expressed as median and interquartile range (mean [IQR]) for non-normally distributed continuous variables, mean and standard deviation (mean \pm sd) for normally distributed continuous variables and as numbers and percentages $(n(\%))$ for categorical variables. Non-normally distributed continuous variables were compared with the MannWhitney $U$ test, categorical variables with the Pearson $\chi^{2}$ test.

Diurnal rhythms of cardiac troponin $\mathrm{T}$ and cystatin $\mathrm{C}$ were analysed by fitting the data to a cosine curve by using the method of cosinor rhytmometry ${ }^{14-16,20,21}$. The cosinor model is described as: $Z(t)=M+A \cdot \cos (\omega t+\varphi)+e(t)$; where $Z(t)$ represents the measured concentration at a given time $(t), M$ the mesor (value about which oscillation occurs), $A$ the amplitude (half the difference between the peak and the nadir value), $\omega$ the angular frequency (degrees per unit time with $360^{\circ}$ representing a complete cycle), $\varphi$ the acrophase (timing of maximal value in degrees), and $e(t)$ the error between the cosinor model and the measurement. Evidence of a diurnal rhythm was indicated by an amplitude significantly greater than 0.

A validated mathematical framework on the dynamics of diurnal rhythms based on cosine functions was applied to examine the hypothetical effect of prolonged elimination-times due to impaired renal function on the rhythmic oscillation of cardiac troponin T and cystatin C. This model is extensively discussed elsewhere, and briefly described in the Supplemental Methods ${ }^{16}$.

Cosine curves were expressed as deviation (\%) from the $24 \mathrm{~h}$ mesor concentration. Given a mean relative cardiac troponin T amplitude of $14 \pm 6 \%$ in the reference group ${ }^{15}$ and assuming a mean decrease in glomerular filtration rate of $80 \%$ in the subjects with impaired renal function, the inclusion of 18 subjects per group would afford $80 \%$ power at a significance level of $5 \%$ to detect a significantly decreased amplitude. The relative amplitudes between both groups were compared using an unpaired T-test. Additionally, a Wald test was 
performed to examine differences in the amplitudes after plotting cosine curves on group level. To test an overall correlation between renal function and the relative amplitude, we performed a Pearson correlation.

All hypothesis testing was two-tailed, and p-values less than 0.05 were considered statistically significant. Statistical analyses were performed with SPSS for Windows 23.0 (IBM Corp.) and R version 3.3.1 and the R-package Cosinor version 1.1 (R-project.org) ${ }^{21}$.

\section{Results}

\section{Prolonged elimination time is predicted to blunt the rhythmic oscillations}

We mathematically modelled the effect of decreased elimination on the amplitude of the diurnal rhythm in cardiac troponin T that was detected in subjects without CKD (individual curves of subjects without CKD have been published previously $\left.{ }^{14,15}\right)$. As depicted in Figure 2, restricted elimination of cardiac troponin T would increase elimination time and fade the circadian rhythm: The relative amplitude of $12 \%$ would diminish to $11 \%, 8 \%$ and $4 \%$ in case of a $60 \%, 80 \%$ and $90 \%$ decrease in elimination of cardiac troponin $\mathrm{T}$, respectively.

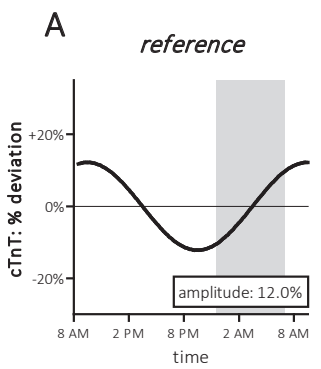

\section{B} $-60 \%$ elimination

C $-80 \%$ elimination
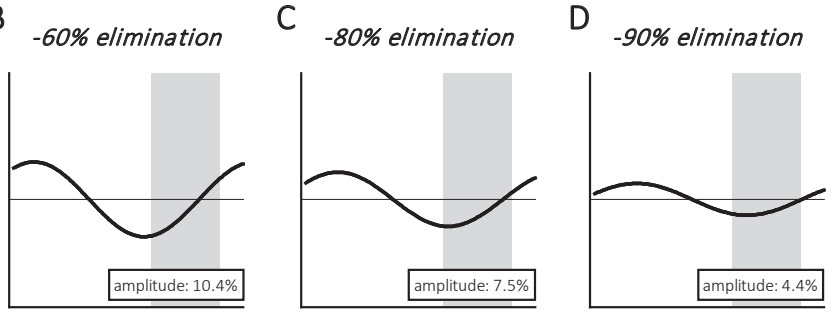

Figure 2. Predicted effect of decreased elimination on rlative amplitudes and circadian rhythm of cardiac troponin T. Fitted cosine curves of 25-hour profile of the percentile deviation from the mesor $24 \mathrm{~h}$ cardiac troponin T (cTnT) concentration (in \%) of 20 reference subjects (panel A) and the predicted cruves for decreased elimination rate (panels B-D).

Diurnal rhythm of cardiac troponin T remains intact in patients with decreased renal function

To examine whether the elimination rate of cardiac troponin $\mathrm{T}$ is decreased, and thus the predicted effect is evident in subjects that truly have decreased renal elimination rates, the 
diurnal rhythm in cardiac troponin T concentrations was analysed in 24 participants with decreased renal function. Participants' baseline characteristics (8.30 AM day 1) are shown in Table 1. Baseline eGFR was by definition lower (median eGFR 18.3 [14.2 - 32.2] vs 82.8 [68.0 - 92.7] $\mathrm{ml} / \mathrm{min} / 1.73 \mathrm{~m}^{2}$ ), whereas baseline cardiac troponin T was significantly higher in the group with CKD, than in the group without CKD disease (median cardiac troponin T 20.5 [18.0 - 24.6] vs. 14.1 [10.4 - 20.6] ng/L, p=0.009).

As shown in the Supplemental Table 1, cardiac troponin T concentrations over the day fitted a cosinor model with an amplitude significantly larger than 0 in all subjects with decreased renal function (range $R^{2}$ 0.54-0.90, all $p \leq 0.001$ ) with similar patterns to those that were observed in the reference population: Gradually decreasing concentrations throughout daytime and rising concentrations during night-time. The observed diurnal rhythm was not related to changes in blood pressure, nor abrogated by the use of loop diuretics (Supplemental Figures 1 and 2).

Table 1. Baseline characteristics

\begin{tabular}{llll}
\hline & $\begin{array}{l}\text { Reference } \\
\mathrm{n}=20\end{array}$ & $\begin{array}{l}\text { Decreased renal function } \\
\mathrm{n}=24\end{array}$ & $\mathrm{p}^{- \text {value }^{*}}$ \\
\hline Age, $\mathrm{y}$ & $72[67-78]$ & $69[61-77]$ & 0.25 \\
Male sex & $16(80)$ & $17(71)$ & 0.49 \\
Antihypertensive medication & $8(40)$ & $16(67)$ & 0.08 \\
Lipid lowering medication & $10(50)$ & $16(58)$ & 0.27 \\
Diabetes Mellitus & $6(30)$ & $8(33)$ & 0.82 \\
Body-mass index, $\mathrm{kg} / \mathrm{m}^{2}$ & $26.3[24.7-29.7]$ & $26.8[24.8-28.5]$ & 0.94 \\
eGFR, ml.min ${ }^{-1} .1 .73 \mathrm{~m}^{-2}$ & $82.8[68.0-92.7]$ & $18.3[14.2-32.2]$ & $<0.001$ \\
Creatinine, $\mu \mathrm{mol} / \mathrm{L}$ & $83.5[68.0-89.8]$ & $256.5[155.0-329.3]$ & $<0.001$ \\
Cystatin C, $\mathrm{mg} / \mathrm{L}$ & $0.94[0.82-1.10]$ & $2.75[1.84-3.51]$ & $<0.001$ \\
Cardiac troponin T, $\mathrm{ng} / \mathrm{L}$ & $14.1[10.4-20.6]$ & $20.5[18.0-24.6]$ & 0.009 \\
NT-proBNP, pmol/L & $9.8[4.4-24.4]$ & $61.5[17.9-102.7]$ & $<0.001$
\end{tabular}

eGFR denotes estimated glomerular filtration rate according to the Chronic Kidney Disease-EPI Creatinine-Cystatin C equation ${ }^{19}$; NT-proBNP, N-terminal prohormone of brain natriuretic peptide. Data is presented as median [IQR] or $\mathrm{n}(\%)$. ${ }^{*}$ Comparisons between reference and subjects with decreased renal function. 


\section{The amplitude of cardiac troponin $\mathrm{T}$ is not dependent on renal function}

The mean relative amplitude was not significantly different between both groups (reference: $13.2[9.1-20.5] \%$, decreased renal function: $11.6[9.0-15.2] \%, p=0.45)$. Also when data was plot and compared on group level, we observed no significant difference between the subjects with or without CKD (Figure 3) (amplitude $12.0 \pm 0.5$ vs. $11.4 \pm 0.5 \%, p=0.53$ ). If an impaired renal function, expressed as a low eGFR, would decrease the elimination rate of cardiac troponin $\mathrm{T}$, a significant positive correlation between the relative amplitude and eGFR is predicted. However, this is not supported by a combined analysis of both studies (R 0.21 , $\mathrm{p}=0.16$ ) (Supplemental Figure 3).

A

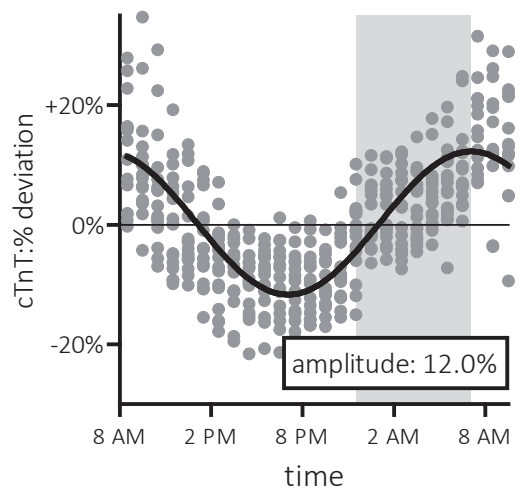

B decreased renal function

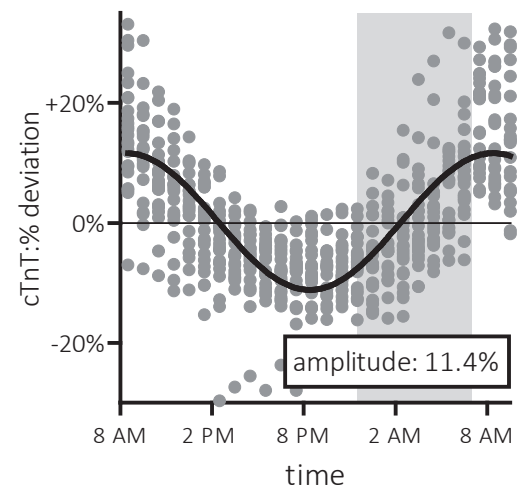

Figure 3. The amplitudes in diurnal troponin T (cTnT) oscillations in subjects with and without decreased renal function. A) All individual data points and cosine curve on the group level of the reference population (CKD $(Z(t)=$ $1.00+0.120 \cdot \cos (0.262 t+25.51)$. All individual data points and cosine curve on the group level for the group with decreased renal function $(C K D(Z(t)=1.00+0.224 \cdot \cos (0.262 t+22.02)$.

Meanwhile, the amplitude of cystatin C, an analyte that also has a diurnal rhythm and with an established renal elimination route 22 , was significantly faded among subjects with decreased renal function compared with the reference group. Its mathematically predicted amplitude in subjects with impaired renal function matched the observed amplitude in these subjects, supporting the validity of the mathematical model (amplitude cystatin c reference group, $3.9 \pm 0.2 \%$ vs $1.7 \pm 0.2 \%$ in subjects with impaired renal function $(P<0.001)$, mathematically predicted amplitude, $2 \%)$. 


\section{Discussion}

This study examined the diurnal rhythm in cardiac troponin T and its relation to the estimated glomerular filtration rate in subjects with or without CKD. We report three major findings. First, cardiac troponin T concentrations exhibit a similar diurnal rhythm in all subjects, with or without CKD. This rhythm is characterized by gradually decreasing concentrations throughout the day and rising concentrations during night-time, and is in line with the circadian pattern of several processes in the cardiovascular system, for example reflected by an increased intensity of cardiovascular events in the morning hours ${ }^{23,24}$.

Second, and most important, there is no fading of the circadian rhythm in subjects with impaired renal function. Decreased elimination of cardiac troponin $T$ and the consecutive prolongation of the terminal elimination time should lead to a faded circadian rhythm with a substantially decreased relative amplitude, as shown by our mathematical model (Figure 2). Whereas previous studies have shown that terminal elimination times of several, endogenous and exogenous, compounds can be prolonged up to more than ten times in patients with $C K D^{25,26}$, we expected to find an obvious decrease in relative amplitude, like we did for cystatin C, if impaired renal clearance would be the main driver behind the elevated cardiac troponin concentrations. The fact that we did not find a difference strongly suggests that the elevated cardiac troponin concentrations in subjects with a decreased renal function are not only explained by decreased elimination and consecutive accumulation of cardiac troponin T. Therefore, our observations do not support the hypothesis that impaired renal elimination is the main driver behind elevated cardiac troponin $T$ levels in subjects with decreased renal function.

Third, we showed a relative amplitude in the diurnal variation of cardiac troponin $T$ of approximately $12 \%$, irrespective of glomerular filtration rate and basal cardiac troponin $T$ concentration. Although in a previous study the diurnal variation in cardiac troponin $\mathrm{T}$ did not seem to affect the diagnostic accuracy for acute myocardial infarction in general ${ }^{15}$, the rhythm can cause substantial absolute troponin changes in CKD subjects with very high chronic troponin levels, and awareness of the rhythm may benefit accurate interpretation ${ }^{27}$. 
Since we did not detect an effect of decreased renal function on the circadian rhythm of cardiac troponin $\mathrm{T}$, we disprove a common misconception in clinical practice, i.e. that impaired renal clearance of cardiac troponins is the main determinant of their accumulation in subjects with $C K D^{7-9,28}$. Accordingly, our findings strongly suggest that other factors than renal elimination contribute to the increase in cardiac troponins in subjects with a decreased renal function, such as increased release of cardiac troponins from the heart due to (subclinical) myocardial injury ${ }^{29,30}$. This is in line with epidemiological evidence, showing a strong association between decreased renal function and cardiovascular risk ${ }^{10}$. However, we cannot fully exclude impaired renal clearance of cardiac troponin $T$ in subjects with $C_{K}{ }^{31}$. From a clinical perspective our findings are of profound importance for the interpretation of cardiac troponin T levels in patients with decreased renal function. Since our results indicate that elevated levels of cardiac troponin T cannot simply be attributed to only decreased renal elimination, an extensive diagnostic work-up is indicated in all patients with elevated cardiac troponin concentrations irrespective of their estimated glomerular filtration rate ${ }^{4}$.

Potential limitations of our study merit consideration. First, the origin of the rhythmic oscillations we observed is still unknown. We showed that the rhythm is independent of sex, glucose metabolism status, or the use of loop diuretics on the diurnal rhythm. Besides, the circadian pattern of cardiac troponin $T$ is not correlated with blood pressure, levels of cortisol, thyroid stimulating hormone and testosterone ${ }^{15}$. Second, we assumed continuous, non-rhythmic, elimination of cardiac troponin $T$ when modelling the predicted effect of a decreasing glomerular filtration rate on the troponin T amplitude. However, even in the case of rhythmic renal elimination, decreased elimination would lead to accumulation of cardiac troponins and fading of the diurnal pattern ${ }^{16}$. Third, an alternative, but unlikely, scenario that may explain the unchanged amplitude in CKD, is that troponin T oscillations on itself are a tightly regulated phenomenon to maintain organism homeostasis. Such a scenario would allow unchanged amplitudes in CKD even if clearance rate is decreased, if compensated by increased oscillation amplitudes in the production rate in the heart and/or clearance.

In conclusion, cardiac troponin T exhibits a similar diurnal rhythm in subjects with or without CKD. Whereas a severely decreased elimination of cardiac troponin $T$ would lead to 
accumulation of cardiac troponin T and fading of the diurnal rhythm, our results suggest that the elimination of cardiac troponin $T$ is not substantially affected in subjects with CKD. Therefore, it is very unlikely that impaired renal elimination is the main cause of persistently elevated cardiac troponin T levels. 


\section{References}

1. Roffi M, Patrono C, Collet JP, Mueller C, Valgimigli M, Andreotti F, Bax JJ, Borger MA, Brotons C, Chew DP, Gencer B, Hasenfuss G, Kjeldsen K, Lancellotti P, Landmesser U, Mehilli J, Mukherjee D, Storey RF, Windecker S, Baumgartner H, Gaemperli O, Achenbach S, Agewall S, Badimon L, Baigent C, Bueno H, Bugiardini R, Carerj S, Casselman F, Cuisset T, Erol C, Fitzsimons D, Halle M, Hamm C, Hildick-Smith D, Huber K, Iliodromitis E, James S, Lewis BS, Lip GY, Piepoli MF, Richter D, Rosemann T, Sechtem U, Steg PG, Vrints C, Luis Zamorano J and Management of Acute Coronary Syndromes in Patients Presenting without Persistent STSEotESoC. 2015 ESC Guidelines for the management of acute coronary syndromes in patients presenting without persistent ST-segment elevation: Task Force for the Management of Acute Coronary Syndromes in Patients Presenting without Persistent ST-Segment Elevation of the European Society of Cardiology (ESC). Eur Heart J. 2016;37:267-315.

2. Amsterdam EA, Wenger NK, Brindis RG, Casey DE, Jr., Ganiats TG, Holmes DR, Jr., Jaffe AS, Jneid H, Kelly RF, Kontos MC, Levine GN, Liebson PR, Mukherjee D, Peterson ED, Sabatine MS, Smalling RW, Zieman SJ, Members AATF, Society for Cardiovascular A, Interventions and the Society of Thoracic S. 2014 AHA/ACC guideline for the management of patients with non-ST-elevation acute coronary syndromes: executive summary: a report of the American College of Cardiology/American Heart Association Task Force on Practice Guidelines. Circulation. 2014;130:2354-94.

3. Thygesen K, Alpert JS, Jaffe AS, Simoons ML, Chaitman BR, White HD, Joint ESCAAHAWHFTFftUDoMI, Katus HA, Lindahl B, Morrow DA, Clemmensen PM, Johanson P, Hod H, Underwood R, Bax JJ, Bonow RO, Pinto F, Gibbons RJ, Fox KA, Atar D, Newby LK, Galvani M, Hamm CW, Uretsky BF, Steg PG, Wijns W, Bassand JP, Menasche P, Ravkilde J, Ohman EM, Antman EM, Wallentin LC, Armstrong PW, Simoons ML, Januzzi JL, Nieminen MS, Gheorghiade M, Filippatos G, Luepker RV, Fortmann SP, Rosamond WD, Levy D, Wood D, Smith SC, Hu D, Lopez-Sendon JL, Robertson RM, Weaver D, Tendera M, Bove AA, Parkhomenko AN, Vasilieva EJ and Mendis S. Third universal definition of myocardial infarction. Circulation. 2012;126:2020-35.

4. deFilippi CR and Herzog CA. Interpreting Cardiac Biomarkers in the Setting of Chronic Kidney Disease. Clin Chem. 2017;63:59-65.

5. Flores-Solis LM and Hernandez-Dominguez JL. Cardiac troponin I in patients with chronic kidney disease stage 3 to 5 in conditions other than acute coronary syndrome. Clin Lab. 2014;60:281-90.

6. Freda BJ, Tang WH, Van Lente F, Peacock WF and Francis GS. Cardiac troponins in renal insufficiency: review and clinical implications. J Am Coll Cardiol. 2002;40:2065-71.

7. Cardinaels EP, Altintas S, Versteylen MO, Joosen IA, Jellema LJ, Wildberger JE, Das M, Crijns HJ, Bekers O, van Dieijen-Visser MP, Kietselaer BL and Mingels AM. High-Sensitivity Cardiac Troponin Concentrations in Patients with Chest Discomfort: Is It the Heart or the Kidneys As Well? PLoS One. 2016;11:e0153300.

8. Diris JH, Hackeng CM, Kooman JP, Pinto YM, Hermens WT and van Dieijen-Visser MP. Impaired renal clearance explains elevated troponin T fragments in hemodialysis patients. Circulation. 2004;109:23-5.

9. Tsutamoto T, Kawahara C, Yamaji M, Nishiyama K, Fujii M, Yamamoto T and Horie M. Relationship between renal function and serum cardiac troponin T in patients with chronic heart failure. Eur J Heart Fail. 2009;11:653-8.

10. Go AS, Chertow GM, Fan D, McCulloch CE and Hsu CY. Chronic kidney disease and the risks of death, cardiovascular events, and hospitalization. N Engl J Med. 2004;351:1296-305.

11. Anavekar NS, McMurray JJ, Velazquez EJ, Solomon SD, Kober L, Rouleau JL, White HD, Nordlander R, Maggioni A, Dickstein K, Zelenkofske S, Leimberger JD, Califf RM and Pfeffer MA. Relation between renal dysfunction and cardiovascular outcomes after myocardial infarction. N Engl J Med. 2004;351:1285-95.

12. Parikh RH, Seliger SL and deFilippi CR. Use and interpretation of high sensitivity cardiac troponins in patients with chronic kidney disease with and without acute myocardial infarction. Clin Biochem. 2015;48:247-53.

13. Aviles RJ, Askari AT, Lindahl B, Wallentin L, Jia G, Ohman EM, Mahaffey KW, Newby LK, Califf RM, Simoons ML, Topol EJ, Berger P and Lauer MS. Troponin T levels in patients with acute coronary syndromes, with or without renal dysfunction. N Engl J Med. 2002;346:2047-52. 
14. Klinkenberg LJ, van Dijk JW, Tan FE, van Loon L, van Dieijen-Visser MP and Meex SJ. Circulating cardiac troponin T exhibits a diurnal rhythm. J Am Coll Cardiol. 2014;63:1788-95.

15. Klinkenberg LJ, Wildi K, van der Linden N, Kouw IW, Niens M, Twerenbold R, Rubini Gimenez M, Puelacher C, Daniel Neuhaus J, Hillinger P, Nestelberger T, Boeddinghaus J, Grimm K, Sabti Z, Bons JA, van Suijlen JD, Tan FE, Ten Kate J, Bekers O, van Loon LJ, van Dieijen-Visser MP, Mueller C and Meex SJ. Diurnal Rhythm of Cardiac Troponin: Consequences for the Diagnosis of Acute Myocardial Infarction. Clin Chem. 2016.

16. Luck S, Thurley K, Thaben PF and Westermark PO. Rhythmic degradation explains and unifies circadian transcriptome and proteome data. Cell Rep. 2014;9:741-51.

17. Levey AS, Stevens LA and Coresh J. Conceptual model of CKD: applications and implications. Am J Kidney Dis. 2009;53:S4-16.

18. Giannitsis E, Kurz K, Hallermayer K, Jarausch J, Jaffe AS and Katus HA. Analytical validation of a high-sensitivity cardiac troponin T assay. Clin Chem. 2010;56:254-61.

19. Inker LA, Schmid CH, Tighiouart $\mathrm{H}$, et al. Estimating glomerular filtration rate from serum creatinine and cystatin C. N Engl J Med 2012;367:20-9.

20. Nelson W, Tong YL, Lee JK and Halberg F. Methods for cosinor-rhythmometry. Chronobiologia. 1979;6:305-23.

21. Tong YL. Parameter estimation in studying circadian rhythms. Biometrics. 1976;32:85-94.

22. Larsson A, Hassan M, Ridefelt P and Axelsson J. Circadian variability of bilirubin in healthy men during normal sleep and after an acute shift of sleep. Chronobiol Int. 2009; 26: 1613-21.

23. Sato M, Matsuo T, Atmore H and Akashi M. Possible contribution of chronobiology to cardiovascular health. Front Physiol. 2013;4:409.

24. Manfredini R, Boari B, Salmi R, Fabbian F, Pala M, Tiseo R and Portaluppi F. Twenty-four-hour patterns in occurrence and pathophysiology of acute cardiovascular events and ischemic heart disease. Chronobiol Int. 2013;30:6-16.

25. Feneberg R, Sparber M, Veldhuis JD, Mehls O, Ritz E and Schaefer F. Altered temporal organization of plasma insulin oscillations in chronic renal failure. J Clin Endocrinol Metab. 2002;87:1965-73.

26. Poschel KA, Bucha E, Esslinger HU, Nortersheuser P, Jansa U, Schindler S, Nowak G and Stein G. Pharmacodynamics and pharmacokinetics of polyethylene glycol-hirudin in patients with chronic renal failure. Kidney Int. 2000;58:2478-84.

27. van der Linden N, Cornelis T, Klinkenberg LJ, Kimenai DM, Hilderink JM, Litjens EJ, Kooman JP, Bekers O, van Dieijen-Visser MP and Meex SJ. Strong diurnal rhythm of troponin T, but not troponin I, in a patient with renal dysfunction. Int J Cardiol. 2016;221:287-8.

28. Bjurman C, Petzold M, Venge P, Farbemo J, Fu ML and Hammarsten O. High-sensitive cardiac troponin, NTproBNP, hFABP and copeptin levels in relation to glomerular filtration rates and a medical record of cardiovascular disease. Clin Biochem. 2015;48:302-7.

29. Masson S, Latini R, Mureddu GF, Agabiti N, Miceli M, Cesaroni G, Forastiere F, Wienhues-Thelen UH, Block D, Zaugg C, Vago T, Boccanelli A and study P. High-sensitivity cardiac troponin T for detection of subtle abnormalities of cardiac phenotype in a general population of elderly individuals. J Intern Med. 2013;273:306-17.

30. Irfan A, Twerenbold R, Reiter M, Reichlin T, Stelzig C, Freese M, Haaf P, Hochholzer W, Steuer S, Bassetti S, Zellweger C, Freidank H, Peter F, Campodarve I, Meune C and Mueller C. Determinants of high-sensitivity troponin T among patients with a noncardiac cause of chest pain. Am J Med. 2012;125:491-498 e1.

31. Friden V, Starnberg K, Muslimovic A, Ricksten SE, Bjurman C, Forsgard N, Wickman A and Hammarsten O. Clearance of cardiac troponin T with and without kidney function. Clin Biochem. 2017. 


\section{Supplemental information}

\section{Supplemental Methods}

\section{Mathematical modeling}

To study the effect of prolonged elimination-times on the observed rhythm of diurnal variation we applied a mathematical framework on the dynamics of diurnal rhythms. Since this model is extensively discussed elsewhere ${ }^{16}$, we just explain it briefly in this section. The applied model assumes a cosineshaped function underlying the circadian rhythm (an assumption that is met for cardiac troponin $\mathrm{T}^{15}$ ), described as: $Z(t)=M+A \cdot \cos (\omega t-\varphi)$, in which $Z(t)$ represents the measured concentration of cardiac troponin $\mathrm{T}$ at a given time $(t), M$ the mesor (the average value on cosine curve fitted to the data), $A$ the amplitude (half the difference between the peak and the nadir value), $\omega$ the angular frequency (degrees per unit time, with $360^{\circ}$ representing $24 \mathrm{~h}$ ) and $\varphi$ the acrophase (timing of maximal value in degrees).

When the observed diurnal rhythm is considered as the resultant of production and degradation, these observed diurnal variation can be expressed as combination of two cosine-shaped functions: $\frac{d x}{d t}=$ $\kappa\left(1+A_{\text {prod }} \cos \left(\omega t-\varphi_{\text {prod }}\right)\right)-\gamma\left(1+A_{\text {deg }} \cos \left(\omega t-\varphi_{\text {deg }}\right)\right) x(t)$, where $\kappa$ represents the mean production coefficient, and $\gamma$ the mean degradation coefficient. This model leads to the abundance: $x(t) \approx M_{x} \cdot\left(1+A_{x} \cdot \cos \left(\omega t-\Delta \varphi_{x}\right)\right.$. In case of constant degradation $\left(A_{\mathrm{deg}}=0\right), M_{x}=\kappa / \gamma, A_{x}=\gamma$.

$A_{\text {prod }} / \sqrt{\gamma^{2}+\omega^{2}}$ and $\Delta \varphi_{x}=\arctan (\omega / \gamma)$, are the solutions for mean, amplitude and phase, respectively. This formula for the amplitude shows that when the mean degradation rate $\gamma$ decreases, the amplitude in $x$ decreases. It can be shown ${ }^{16}$ that this relationship holds qualitatively also when the degradation rate itself is rhythmic $\left(A_{\text {deg }}>0\right)$. 


\section{Supplemental Figures}
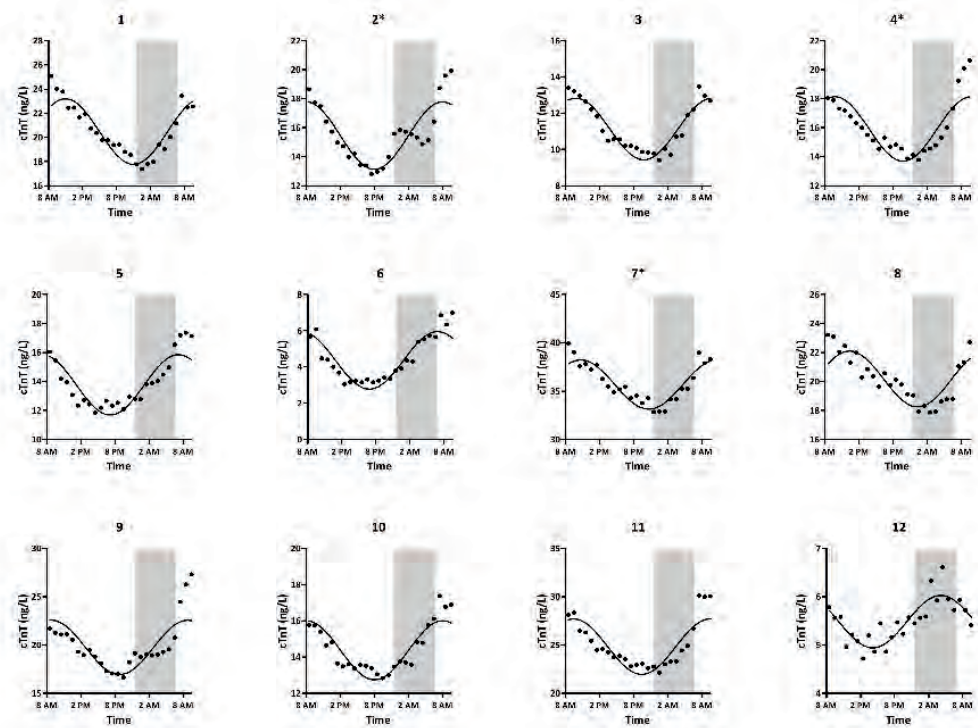

10

11
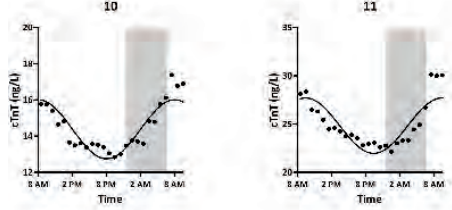

12

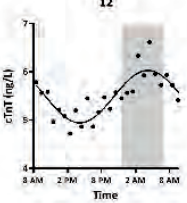

13

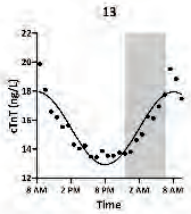

14
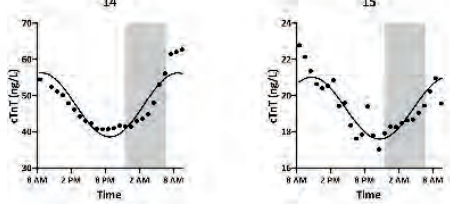

16

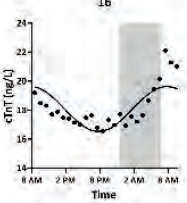

$17 *$
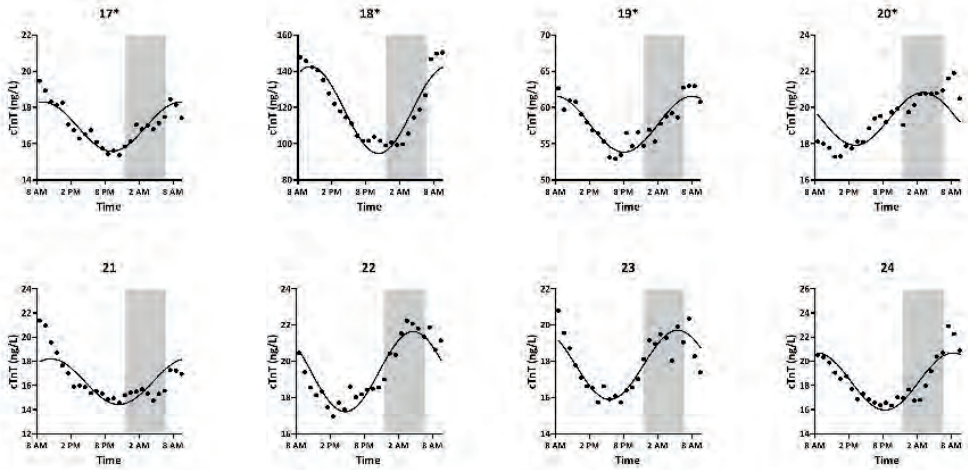

23

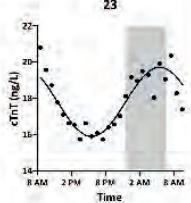

24

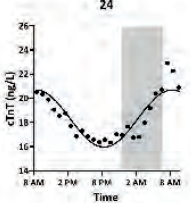

Supplemental figure 1. Individual diurnal cardiac troponin T profiles and fitted cosine curves. Cosine curves for subjects with impaired renal function. Depicted are the mean of the duplicate measurements. The numbering is identical to Table S1. Subjects slept in a supine position between 11.30 PM and 7.00 AM (shaded area). *indicates the use of loop diuretics. 

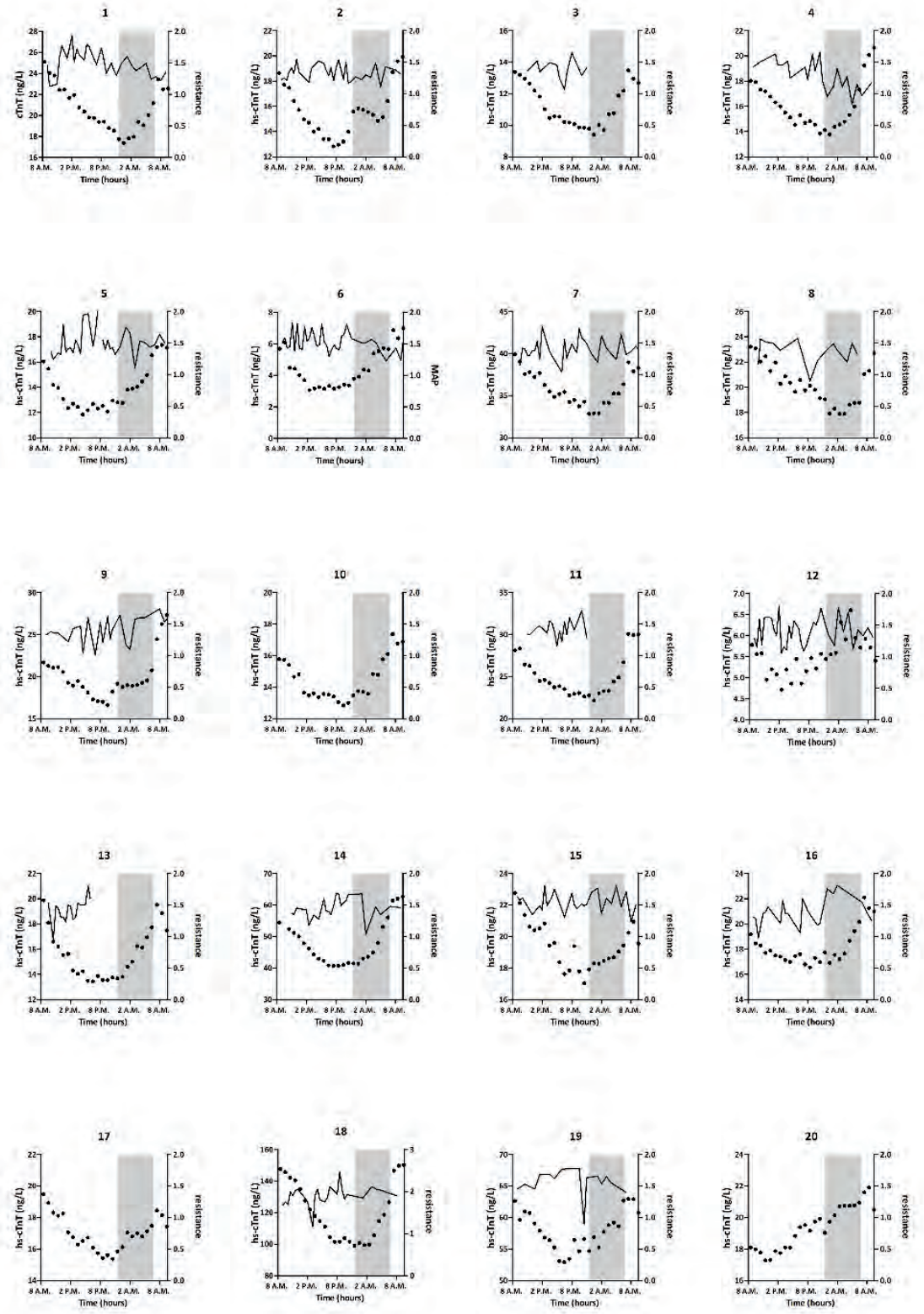

Supplemental Figure 2. Individual diurnal cardiac troponin T profiles and mean arterial pressure. Cosine curves for subjects with decreased renal function. Depicted are the mean of the duplicate measurements. The numbering is identical to Table S1. Subjects slept in a supine position between 11.30 PM and 7.00 AM(shaded area). Arterial pressure data is not available for subjects 21-24. 


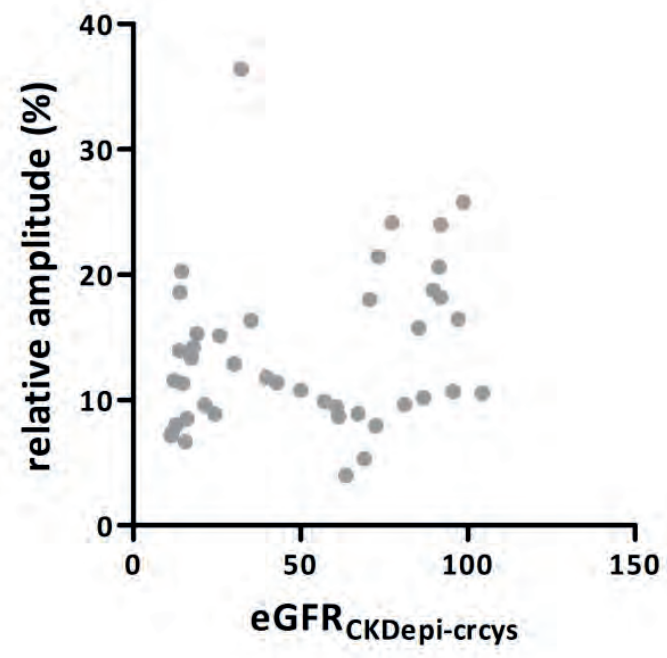

Supplemental figure 3. Correlation between relative amplitudes and kidney function (eGFR). No significant correlation between eGFR and relative amplitude of cardiac troponin T (Pearson's R 0.21, p=0.16). 


\section{Supplemental Tables}

Supplemental table 1. The individual cosinor models for subjects with decreased renal function

\begin{tabular}{|c|c|c|c|c|c|}
\hline Participant & Mesor (ng/L) & Amplitude (ng/L) & Acrophase (h) & $\mathrm{R}^{2}$ & P-value \\
\hline 1 & 20.5 & 2.7 & $11.00 \mathrm{AM}$ & 0.84 & $<0.001^{*}$ \\
\hline 2 & 15.5 & 2.3 & $7.59 \mathrm{AM}$ & 0.73 & $<0.001^{*}$ \\
\hline 3 & 11.1 & 1.7 & $9.46 \mathrm{AM}$ & 0.88 & $<0.001^{*}$ \\
\hline 4 & 15.9 & 2.2 & $9.46 \mathrm{AM}$ & 0.75 & $<0.001^{*}$ \\
\hline 5 & 13.8 & 2.1 & $7.09 \mathrm{AM}$ & 0.77 & $<0.001^{*}$ \\
\hline 6 & 4.4 & 1.6 & $6.52 \mathrm{AM}$ & 0.83 & $<0.001^{*}$ \\
\hline 7 & 35.7 & 2.5 & 10.39 AM & 0.83 & $<0.001^{*}$ \\
\hline 8 & 20.2 & 1.9 & 12.19 PM & 0.69 & $<0.001^{*}$ \\
\hline 9 & 19.7 & 2.8 & $8.47 \mathrm{AM}$ & 0.63 & $<0.001^{*}$ \\
\hline 10 & 14.4 & 1.6 & $8.00 \mathrm{AM}$ & 0.81 & $<0.001^{*}$ \\
\hline 11 & 24.8 & 2.9 & $9.25 \mathrm{AM}$ & 0.75 & $<0.001^{*}$ \\
\hline 12 & 5.5 & 0.5 & 4.11 AM & 0.72 & $<0.001^{*}$ \\
\hline 13 & 15.4 & 2.5 & $8.02 \mathrm{AM}$ & 0.86 & $<0.001^{*}$ \\
\hline 14 & 47.4 & 8.8 & 8.29 AM & 0.83 & $<0.001^{*}$ \\
\hline 15 & 19.3 & 1.7 & 10.37 AM & 0.74 & $<0.001 *$ \\
\hline 16 & 18.1 & 1.5 & $7.44 \mathrm{AM}$ & 0.60 & $<0.001^{*}$ \\
\hline 17 & 16.9 & 1.3 & 9.12 AM & 0.82 & $<0.001^{*}$ \\
\hline 18 & 118.6 & 23.9 & 10.14 AM & 0.90 & $<0.001^{*}$ \\
\hline 19 & 57.7 & 3.9 & $8.00 \mathrm{AM}$ & 0.85 & $<0.001 *$ \\
\hline 20 & 19.4 & 1.5 & $3.05 \mathrm{AM}$ & 0.54 & $<0.001 *$ \\
\hline 21 & 16.3 & 1.9 & 10.26 AM & 0.57 & $<0.001^{*}$ \\
\hline 22 & 19.4 & 2.2 & $4.35 \mathrm{AM}$ & 0.86 & $<0.001^{*}$ \\
\hline 23 & 17.8 & 1.9 & 5.29AM & 0.79 & $<0.001^{*}$ \\
\hline 24 & 18.3 & 2.4 & $8.19 \mathrm{AM}$ & 0.82 & $<0.001^{*}$ \\
\hline
\end{tabular}

The cosinor model is described as: $Z(t)=M+A \cdot \cos (\omega t+\varphi)+e(t)$. where $Z(t)$ represents the measured cardiac troponin $T$ concentration at a given time $(t)$. $M$ the mesor (value about which oscillation occurs). $A$ the amplitude (half the difference between the peak and the nadir value). $\omega$ the angular frequency (degrees per unit time. with $360^{\circ}$ representing a complete cycle). $\varphi$ the acrophase (timing of maximal value in degrees) and $e(t)$ the error between the cosinor model and the measurement. *Significant after Bonferroni correction for multiple testing. $p=0.0025(0.05 / 20)$. 
Cardiac troponins: State of the (He)Art 


\section{Mass spectrometric identification of}

\section{cardiac troponin $T$ in urine of patients}

\section{suffering from acute myocardial infarction}

Sander Streng*, Noreen van der Linden*, Jordy Kocken, Otto Bekers, Freek Bouwman, Edwin Mariman, Steven Meex, Will Wodzig, Douwe de Boer - J App/Lab Med 


\section{Abstract}

\section{Background}

Due to its high cardiospecificity, cardiac troponin $\mathrm{T}$ (cTnT) is one of the biomarkers of first choice to diagnose acute myocardial infarction (AMI). CTnT is extensively fragmented in serum of patients suffering from AMI. However, it is as of yet unknown whether all cTnT is completely degraded in the body or if some CTnT fragments can leave the body via the urine. The aim of the present study is to develop a method for the detection of $\mathrm{CTnT}$ in urine and to examine whether CTnT is detectable in patient urine.

\section{Methods}

Proteins in urine samples of twenty patients were precipitated using a cTnT-specific immunoprecipitation technique and a non-specific acetonitrile protein precipitation. After in-solution digestion of the precipitated proteins, the resulting peptides were separated and analysed using highperformance liquid chromatography and mass spectrometry using a targeted selected ion monitoring assay with data-dependent tandem-MS (t-SIM/dd-MS2).

\section{Results}

The t-SIM/dd-MS2 assay was validated using a synthetic peptide standard containing ten specific cTnT peptides of interest and with purified human intact cTnT spiked in urine from healthy individuals. Using this assay, a total of six different cTnT-specific peptides were identified in urine samples from three different patients, all suffering from AMI.

\section{Conclusions}

We show here for the first time that CTnT can be present in the urine of patients suffering from AMI using a targeted LC-MS/MS assay. Whether the presence of CTnT in urine reflects a physiological or rather a pathophysiological process still needs to be elucidated. 


\section{Introduction}

Cardiac troponin $\mathrm{T}(\mathrm{cTnT})$ is one of the preferred biomarkers to establish the diagnosis of acute myocardial infarction (AMI) without characteristic electrocardiogram changes in addition to a clinical presentation and physical examination ${ }^{1-3}$. Nevertheless, the interpretation of cardiac troponin concentrations can be challenging, especially in subjects with persistently elevated cardiac troponin levels ${ }^{4,5}$, like patients suffering from chronic kidney disease. The high prevalence of persistently elevated cardiac troponin concentrations among subjects with impaired renal function has raised the question if, and if so how, the kidneys are involved in the terminal elimination of cardiac troponins ${ }^{6-8}$. A better understanding of the final elimination of cardiac troponins might help us in correctly interpreting cardiac troponin concentrations.

Previous studies have shown that the initial release of cTnT in response to cardiomyocyte damage ${ }^{9,10}$ is followed by a prolonged increase in cTnT levels that can persist for 7-14 days ${ }^{10-}$ ${ }^{12}$. In addition, it has been shown that CTnT is highly degraded in serum of patients suffering from $\mathrm{AMI}$ in a time-dependent manner ${ }^{12,13}$. However, the next step in the elimination of these cTnT fragments is still unclear. One possible hypothesis is that the smallest cTnT fragments are further degraded in the circulation until they are no longer detectable by the clinical assay. Another hypothesis is that cTnT fragments are eliminated via the kidneys ${ }^{12,14}$. Based on their size and charge, the cTnT fragments are susceptible to glomerular filtration ${ }^{15}$, and should normally be degraded and reabsorbed by the proximal tubule $\mathrm{e}^{16,17}$. These filtered cTnT fragments may also be excreted via the urine, for example due to dysfunction or overload of the tubuli as a result of proteinuria ${ }^{18}$.

Recently, we have developed a gel-based targeted mass spectrometry assay for the identification of cTnT and its fragments in serum ${ }^{19-21}$. In the present study, we have modified and validated this assay for the analysis of urine samples. We explored two different sample workup methods and applied them on urine samples of patients with acute myocardial injury. 


\section{Methods}

\section{Reagents, synthetic peptide standard, and positive control}

UPLC-grade water, acetonitrile (ACN), formic acid (FA), and trifluoroacetic acid (TFA) for sample preparation and mass spectrometry were obtained from Biosolve (Valkenswaard, the Netherlands). Ammonium bicarbonate (AMBIC), $\geq 99.5 \%$, was from Sigma-Aldrich (St. Louis,

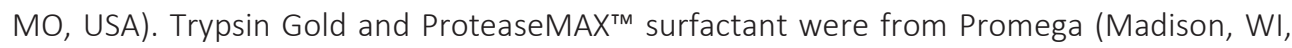
USA).

Ten tryptic cTnT peptides of interest were synthesised by Pepscan (Lelystad, the Netherlands) with an average purity $>95 \%$. These peptides were dissolved in $20 \%$ ACN and subsequently pooled in $2.0 \% \mathrm{ACN}, 0.01 \%$ TFA at a concentration of $40 \mathrm{nmol} / \mathrm{L}$ to create a synthetic peptide standard. This standard was used as a quality control sample throughout the experiment and was also used to schedule the targeted MS experiments.

Validation experiments were performed with a positive control consisting of purified human intact cTnT (cat\# 8T13, HyTest LTD, Turku, Finland) spiked in both 50 mM AMBIC and fresh urine from a healthy volunteer in various concentrations.

\section{Urine samples}

Twenty urine samples from different patients suffering from acute myocardial injury (STelevation myocardial infarction (STEMI) $(n=9)$, non ST-elevation myocardial infarction (NSTEMI) $(n=4)$, myocarditis $(n=5)$, cardiac arrest $(n=1)$ or miscellaneous $(n=1))$ submitted for routine diagnostic analysis were collected over the course of one year when a concomitant cTnT-measurement in the serum of that patient was $>1000 \mathrm{ng} / \mathrm{L}$. Supplemental table 1 shows the relevant patient characteristics. Urine samples were stored for a maximum of 7 days at $4{ }^{\circ} \mathrm{C}$, after which they were aliquoted and stored at $-80^{\circ} \mathrm{C}$ until analysis. After thawing of samples, urine was first centrifuged at 2,000 g for $10 \mathrm{~min}$ and subsequently treated as described below. Patient samples were randomised and treated according to the code of proper secondary use of human tissue in the Netherlands (www.fmwv.nl). As a control, 19 urine samples were collected from presumably healthy volunteers who did not report 
symptoms related to acute myocardial infarction neither had a medical history of cardiovascular disease.

\section{Sample workup; protein concentration and digestion}

In this study, all samples were analysed using both immunoprecipitation and ACN precipitation to concentrate CTnT from urine. Both methods were followed by in-solution digestion and mass spectrometry. Immunoprecipitation of cTnT in $400 \mu \mathrm{L}$ urine was performed by the addition of $50 \mu \mathrm{L}$ M-270 Streptavidin Dynabeads (Invitrogen, Carlsbad, CA, USA) coupled to the biotinylated anti-cTnT antibody M11.7 (Roche Diagnostics, Basel, Switzerland) ${ }^{22}$. After incubation for 1 hour at room temperature, immunoprecipitated proteins were eluted in $50 \mathrm{mM} \mathrm{AMBIC}, \mathrm{pH} 10$, for $15 \mathrm{~min}$ at $56^{\circ} \mathrm{C}$, followed by titration to $\mathrm{pH}$ 8. As an alternative to immunoprecipitation, ACN precipitation was performed by the addition of $1400 \mu \mathrm{L}$ ice-cold $\left(-20^{\circ} \mathrm{C}\right) \mathrm{ACN}$ to $400 \mu \mathrm{L}$ urine and incubation at $-20^{\circ} \mathrm{C}$ overnight. Samples were centrifuged at $12,000 \mathrm{~g}$ for $30 \mathrm{~min}$ at $4{ }^{\circ} \mathrm{C}$ and the supernatant was decanted. Pellets were washed in $1 \mathrm{~mL}$ ice-cold ACN, air dried, and redissolved in $50 \mu \mathrm{L}$ AMBIC $(50 \mathrm{mM}$, $\mathrm{pH}$ 8). The digestion of all samples was performed with $1 \mu \mathrm{g}$ Trypsin Gold in the presence of $0.03 \%$ ProteaseMAX ${ }^{\mathrm{TM}}$ surfactant for 3 hours at $37^{\circ} \mathrm{C}$. TFA was added to a final concentration of $0.5 \%$ before LC-MS/MS analysis.

\section{Mass spectrometric analysis of urine samples}

Identification of cTnT presence in urine was performed using an established targeted mass spectrometry assay ${ }^{19,20}$ on a $\mathrm{Q}$ Exactive hybrid quadrupole-Orbitrap mass spectrometer, connected to a UHPLC Dionex Ultimate 3000 (ThermoFisher Scientific, Waltham, MA, USA). Solvent A consisted of $0.1 \%$ formic acid (FA) in $100 \%$ water, solvent B consisted of $0.08 \%$ FA, $20 \%$ water, and 80\% ACN. Peptides were first trapped on an Acclaim PepMap 100, $100 \mu \mathrm{m} x$ $2 \mathrm{~cm}, \mathrm{C} 18,5 \mu \mathrm{m}, 100 \mathrm{~A}$ trap column in 0.1\% TFA, 2\% ACN and 98\% water. Next, peptides were separated on an Acclaim PepMap RSLC, $75 \mu \mathrm{m} \times 15 \mathrm{~cm}, \mathrm{C} 18,2 \mu \mathrm{m}, 100$ A analytical column by a 30 min gradient of $4-55 \%$ solvent $B$. Washout was performed by a 1 min gradient of $55-90 \%$ solvent $B$ and a constant flow of $90 \%$ solvent $B$ for $4 \mathrm{~min}$ at $300 \mathrm{~nL} / \mathrm{min}$. 
Selected ion monitoring (SIM) scans were acquired in the Orbitrap with a width of 2.0 Th at a resolution of 70,000 full width at half maximum (FWHM) at $200 \mathrm{~m} / \mathrm{z}$, automated gain control (AGC) of $10^{5}$, and a maximum injection time of $250 \mathrm{~ms}$. SIM scans were scheduled based on the retention times of different peptides obtained using a full scan of the synthetic standard. Selected $\mathrm{m} / \mathrm{z}$ values were based on the observed peptide masses from the synthetic standard. As a second scan event, a maximum of 10 most intense precursor ions within each SIM scan were selected for higher-energy collisional dissociation (HCD) with an isolation window of 1.0 Th and a normalised collision energy (NCE) that was optimized for each peptide. Product ions were detected in the range of $250-1500 \mathrm{~m} / \mathrm{z}$ at a resolution of $17,500 \mathrm{FWHM}$ at $200 \mathrm{~m} / \mathrm{z}, \mathrm{AGC}$ target of $2 \cdot 10^{5}$, maximum injection time of $200 \mathrm{~ms}$, and a dynamic exclusion window of $30 \mathrm{~s}$.

\section{cTnT immunoreactivity determination}

cTnT-specific immunoreactivity within the collected urine samples was determined with the $5^{\text {th }}$ generation (high sensitivity) CTnT assay on the Cobas ${ }^{\circledR} 6000$ instrument by Roche Diagnostics; the 99th percentile among healthy subjects is $14 \mathrm{ng} / \mathrm{L}$, with an interassay CV between $1 \%$ and $5 \%$ above $30 \mathrm{ng} / \mathrm{L}^{23}$.

The use of the Roche immunoassay in urine was validated by creating a serial dilution series of the positive control spiked in a urine sample of a healthy individual, in triplicate.

\section{Data analysis}

Product ion spectra were searched against the Uniprot Human database dated 13 november 2013 (39,690 entries) using the database search engine SEQUEST with Proteome Discoverer, version 1.8 (ThermoFisher). Precursor and product ion mass tolerances were set to $10 \mathrm{ppm}$ and 0.1 Da, respectively. Enzyme specificity was set to semi-tryptic with 2 missed cleavages. Fixed value PSM validator was used using default Xcorr cut-offs. All accepted spectra were manually evaluated. Statistical significance of cTnT concentrations in urine as measured with the $5^{\text {th }}$ generation Roche assay was assessed with a two-tailed Mann-Whitney test where $p$ $<0.05$ was considered statistically significant, using GraphPad Prism, version 5.03. 


\section{Results}

\section{Optimization of sample workup and LC-MS/MS}

The synthetic peptide standard, containing synthesised peptides specific to $c T n T$, was first analysed using a full scan with data-dependent MS/MS (dd-MS2). Figure 1 shows a representative precursor ion chromatogram of this measurement. Every peptide present in the synthetic standard was positively identified using dd-MS2. Even though all peptides were pooled in equimolar amounts, it can be observed that the measured abundance of each peptide differs (for example due to ionisation differences). This analysis was repeated regularly to assess the mass accuracy of the instrument and retention time drift between experiments.

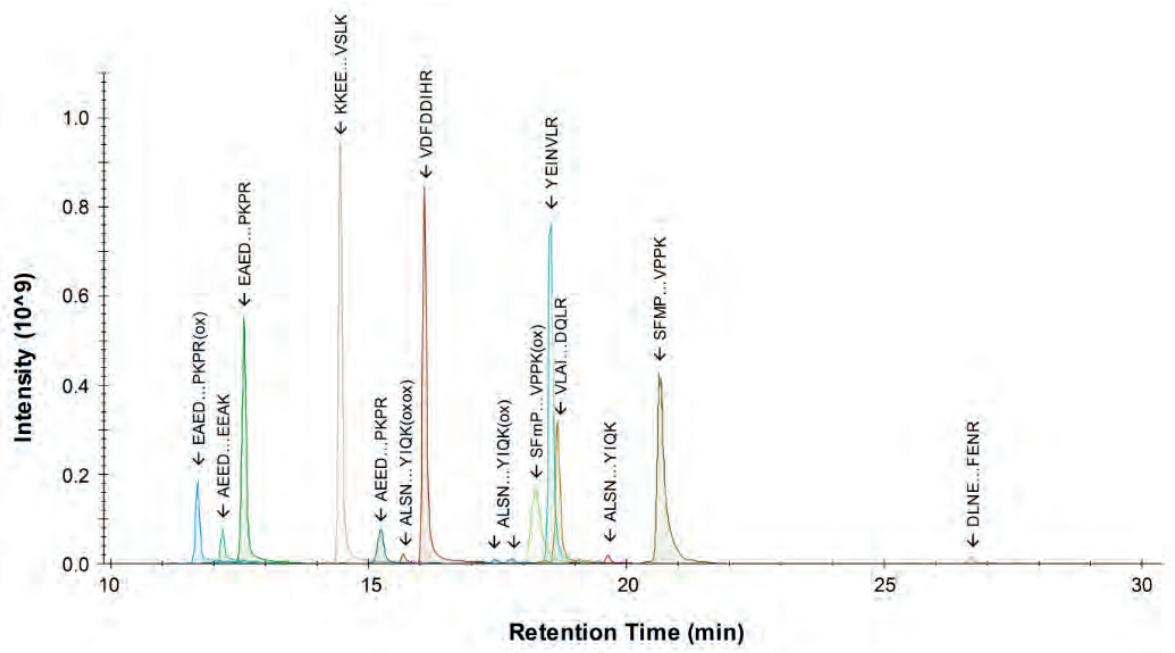

Figure 1. Selected ion chromatogram of the precursor ions of all peptides present in the synthetic peptide standard. The synthetic peptide standard consisted of ten synthesized peptides spiked in equimolar amounts. All labelled peaks were identified with MS/MS. Methionine residues contained within some peptides can be oxidised, designated with: '(ox)'.

Both the immunoprecipitation and the ACN precipitation protocols were validated by serially diluting the positive control in urine from a healthy volunteer and subjecting this dilution series to both workflows. Table 1 lists the peptides identified using MS/MS in both dilution experiments. The listed CTnT concentrations are calculated based on the added amount of 
cTnT and the dilution factors used in both experiments. As shown, both protocols were able to identify cTnT spiked in low concentrations in urine. However, some peptides are identified in lower concentrations than others, while others may not be identified at all. In addition, some of the peptides (like ${ }^{108}$ DLNE...FENR ${ }^{123}$ and ${ }^{228}$ VLAI...DQLR ${ }^{240}$ ) are not detected at all using ACN precipitation, but can be identified in very low concentrations using immunoprecipitation. In general, it can be shown that the limit of identification is lower when using immunoprecipitation than using ACN precipitation.

Table 1. dd-MS2 identification of cTnT peptides of interest in dilution series of positive control samples.

\begin{tabular}{|c|c|c|c|c|c|c|c|c|}
\hline \multirow{2}{*}{$\begin{array}{l}\text { Workflow } \\
\text { Concentration (ng/L) }\end{array}$} & \multicolumn{4}{|c|}{ ACN precipitation } & \multicolumn{4}{|c|}{ Immunoprecipitation } \\
\hline & 3000 & 1500 & 750 & 300 & 5000 & 2500 & 1000 & 500 \\
\hline${ }^{53} \mathrm{AEED} \ldots \mathrm{EEAK}^{63}$ & No & No & No & No & Yes & No & No & No \\
\hline${ }^{53} \mathrm{AEED} \ldots . . \mathrm{PKPR}^{78}$ & Yes & Yes & No & No & Yes & No & No & No \\
\hline${ }^{64}{ }^{6 A E D} \ldots . . . P K P R^{78}$ & Yes & Yes & No & No & Yes & Yes & No & No \\
\hline${ }^{79}$ SFMP...VPPK ${ }^{88}$ & Yes & Yes & No & No & Yes & Yes & Yes & Yes \\
\hline${ }^{95} \mathrm{VDFDDIHR}{ }^{102}$ & Yes & Yes & No & No & Yes & Yes & Yes & Yes \\
\hline${ }^{108}$ DLNE...FENR ${ }^{123}$ & No & No & No & No & Yes & Yes & Yes & Yes \\
\hline${ }^{124}$ KKEE....VSLK $^{134}$ & No & No & No & No & Yes & No & No & No \\
\hline${ }^{187}$ ALSN...YIQK ${ }^{200}$ & Yes & Yes & No & No & Yes & Yes & Yes & No \\
\hline${ }^{228} \mathrm{VLAI}^{2 . . . D Q L R}{ }^{240}$ & No & No & No & No & Yes & Yes & Yes & Yes \\
\hline${ }^{269}$ YEINVLR ${ }^{275}$ & Yes & Yes & Yes & Yes & Yes & Yes & Yes & Yes \\
\hline
\end{tabular}

Purified human intact cTnT was dissolved in urine and serially diluted. Both dilution series were then subjected to either acetonitrile (ACN) precipitation or immunoprecipitation followed by in-solution digestion and mass spectrometry. 'Yes' indicates that the peptide was identified using dd-MS2, 'No' indicates that no such identification was made. Amino acid numbering is based on the canonical adult human isoform of cTnT (CTNT-3, P45379-6).

\section{Troponin identification in patient urine}

Both protocols were applied to the 20 clinical urine samples from patients with acute myocardial injury. When using immunoprecipitation, cTnT specific peptides were identified in two of the analysed samples. In one sample, four peptides could be identified (figure 2a). In the other sample, only one peptide was identified (figure 2 b). When ACN precipitation was 
used, cTnT peptides were identified in a single additional sample (figure 2c). The identification of cTnT in these three samples was independently verified using fresh aliquots in a repeat experiment. No cTnT peptides could be detected with LC-MS/MS in the other 17 urine samples. All peptides were identified with high confidence (xcorr $>1.9$ and $\Delta C n=0$ for all peptides; table 2).

In addition to the LC-MS/MS identifications, CTnT immunoreactivity was measured in all urine samples using the $5^{\text {th }}$ generation Roche assay. Figure $3 a$ shows the measured cTnT immunoreactivity of a dilution series of the positive control in urine of a healthy volunteer $\left(R^{2}=0.9981\right)$, indicating that the assay responds linearly to a serial dilution within the relevant measuring range in urine. Figure $3 \mathrm{~b}$ shows that the immunoreactivity is significantly higher in urine samples from patients suffering from myocardial injury (median $=17.9 \mathrm{ng} / \mathrm{L}$ ) than in urine samples from healthy individuals (median $=3.54 \mathrm{ng} / \mathrm{L}, \mathrm{p}<0.0001$ ). There is no significant correlation between the measured CTnT concentrations in serum and in urine (Spearman's $R^{2}=0.095, p=0.186$, not shown). Supplemental table 1 shows the relevant laboratory measurements of each individual patient included in the study.

Table 2. Cross-correlation (xcorr) and delta correlation $(\Delta \mathrm{Cn})$ values of identified cTnT peptides in patient urine.

\begin{tabular}{cccc}
\hline Patient & Identified peptide & Xcorr & $\Delta$ Cn \\
\hline a & ${ }^{79}$ SFMP...VPPK ${ }^{88}$ & 2.29 & 0 \\
a & ${ }^{95}$ VDFDDIHR ${ }^{102}$ & 2.58 & 0 \\
a & ${ }^{108}$ DLNE...FENR ${ }^{123}$ & 5.65 & 0 \\
a & ${ }^{124}$ KKEE...VSLKK $^{134}$ & 4.55 & 0 \\
b & ${ }^{124}$ KKEE...VSLKK $^{134}$ & 4.45 & 0 \\
c & ${ }^{53}$ AEED...EEAK & \\
& ${ }^{64}$ & 2.08 & 0 \\
c & & 3.48 & 0
\end{tabular}

Xcorr and $\Delta \mathrm{Cn}$ scores are listed for all the spectra in figure 3. Xcorr cutoff value was set to 1.9, maximum $\Delta \mathrm{Cn}$ score allowed was 0.05 . 

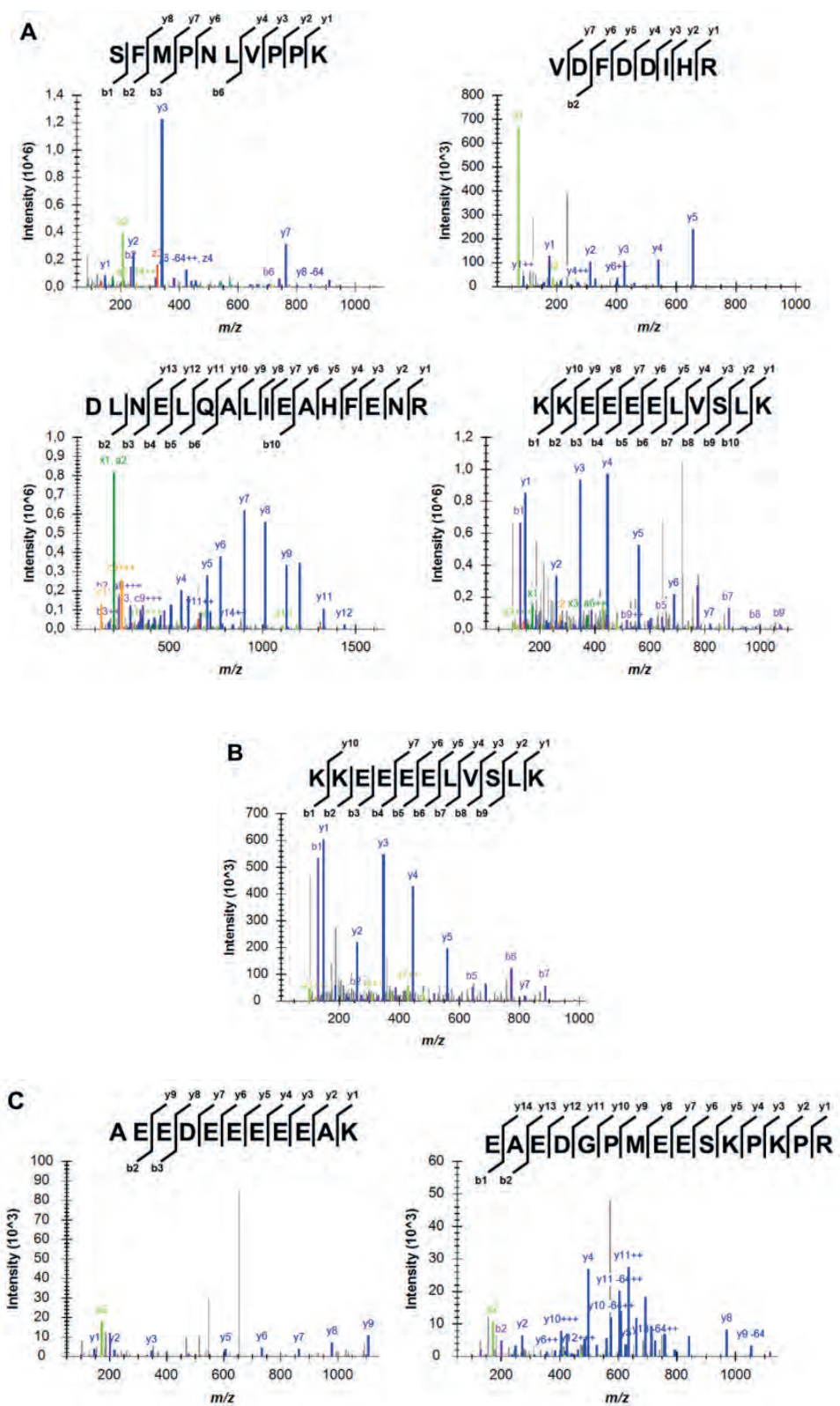

Figure 2. Product ion spectra of cTnT peptides present in patient urine samples. A) Four peptides, located at the centre region of cTnT, were identified in patient (a) using immunoprecipitation. B) One peptide, located at the centre region of cTnT, was identified in patient (b) using immunoprecipitation. C) Two peptides, located at the N-terminus of cTnT, were identified in patient (c) using acetonitrile precipitation. 
A

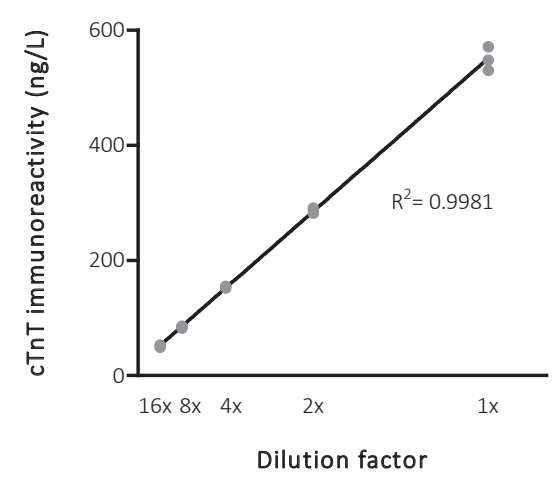

B

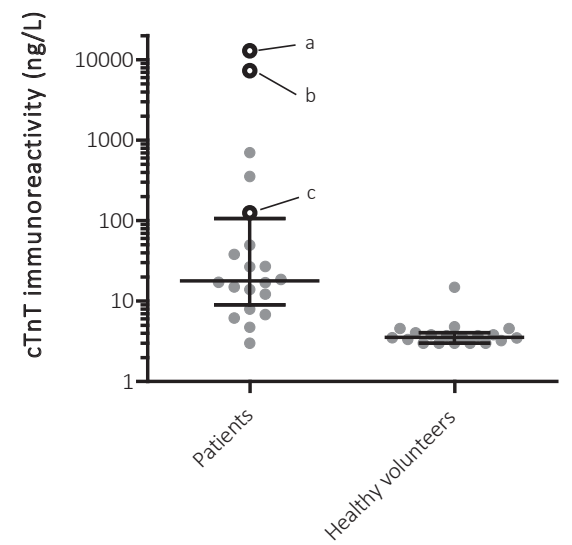

Figure 3. Immunoreactivity in urine samples as measured with the 5 th generation clinical cTnT immunoassay by Roche Diagnostics. A. Serial dilution series of the positive control spiked in urine of a healthy individual. $R 2=0.9981, n=3$. B. Twenty urine samples of patients suffering from acute myocardial injury and of 19 healthy controls. Error bars indicate median and interquartile range, $p<0.0001$. Open dots indicate urine samples where cTnT was positively identified using mass spectrometry, labelled a, b, and c, respectively, as in figure 2, and table 2.

\section{Discussion}

In this study, a targeted LC-MS/MS assay and two different proteomic workflows were used to detect the presence of cardiac troponin $T$ in urine. Using this method, we are the first to provide conclusive evidence that cTnT can be present in the urine of patients with high serum concentrations of cTnT. We were able to show the presence of cTnT-specific peptides in urine in three out of twenty patients suffering from acute myocardial injury. All three of these patients suffered from AMI, with concomitant proteinuria.

While urine is a biological matrix that can be conveniently and non-invasively acquired in large quantities, proteomics-based identification of proteins in urine is a challenge. Urine proteomics is complicated by high interfering concentrations of salts and urea, a broad pH spectrum, and relatively low protein concentrations ${ }^{24}$. Since our primary interest was the identification of cTnT in urine, we selectively captured cTnT from urine using an adapted immunoprecipitation technique based on a successful protocol for cTnT isolation from serum ${ }^{12,19,22}$. This technique removes salts, urea, and other interfering components, but is 
very selective in its binding to the central region of cTnT. The peptides identified with this workflow (figures $2 \mathrm{a}$ and $2 \mathrm{~b}$ ) indeed do originate from this central region (figure 4). Since CTnT is subject to proteolytic degradation in the blood circulation 12,13,21,25, it is possible that this methodology is too selective and that CTnT fragments that do not contain this central region might be missed. For this reason, we repeated the experiment using a non-selective workflow based on the precipitation of proteins using an organic solvent ${ }^{24,26-29}$. This resulted in the identification of two peptides originating from the $\mathrm{N}$-terminus (which removal is considered to be the first step in the degradation of $\mathrm{cTnT}^{12,21}$ ) in an additional sample (figure 2c, figure 4). The peak serum concentration of cTnT in this particular patient was very high $(31,199 \mathrm{ng} / \mathrm{L}$, supplemental table 1), which may explain why we identified cTnT peptides in the urine of this patient, despite the fact that the measured cTnT immunoreactivity in his urine was much lower (125 ng/L). The other two patients in which cTnT peptides were identified had a very high cTnT-specific immunoreactivity in urine $(13,000$ and 7,365 ng/L; red dots in figure $3 b$ ), supporting the hypothesis that the limit of identification of the tSIM/dd-MS2 assay was insufficient to detect cTnT peptides in the other urine samples. Another possibility is that the cTnT present in urine is even more progressively degraded compared to serum. When the arginine and lysine rich centre region is the only part of cTnT that remains in urine, it may become completely destroyed during tryptic digestion and will be missed with the t-SIM/dd-MS2 assay, but possibly not with the immunoassay, where digestion is not performed. Because we utilized leftover samples from patients routinely analysed in our laboratory, no protease inhibitors or other stabilizers were added to the urine upon collection. Therefore, it is possible that any fragmentation of cTnT continues in urine. For the same logistic reason, the urine samples were not collected on standardized time points and no serial sampling was performed. Future research should take these suggestions into account. 
24-33: Peptide spliced in the adult human isoform

(c)

\section{MSDIEEVVEE YEEEEQEEAA VEEEEDWRED EDEQEEAAEE DAEAEAETEE TRAEEDEEEE}

\section{(c) \\ (อ) \\ (3) \\ (2) \\ 61 EAKEAEDGPM EESKPKPRSF MPNLVPPKIP DGERVDFDDI HRKRMEKDLN ELQALIEAHF}

(a)b) 135-141, 146-157: Roche $M 7$ and $M 11.7$ antibody epitopes, respectively

121 ENRKKEEEEL VSLKFRIERR RJAERAEQQRI RNEREKERQN RLAEERARRE EEENRRKAED

181 EARKKKALSN MMHFGGYIQK QAQTERKSGK RQTEREKKKK ILAERRKVLA IDHLNEDQLR

\section{EKAKELWQSI YNLEAEKFDL QEKFKQQKYE INVLRNRIND NQKVSKTRGK AKVTGRWK 298}

Figure 4. Annotated sequence of the canonical isoforms of cardiac troponin T. Green highlights indicate peptides that were successfully identified using mass spectrometry in patients (a), (b), and (c), as depicted. Red highlights indicate targeted peptides that were not identified in any of the samples. The orange highlighted area indicates a region that is spliced in the adult human cTnT isoforms (isoforms 6, cTnT-3). The purple highlights indicate the epitopes of the Roche M7 detector and M11.7 catcher antibodies.

It is important to note that we were only able to identify cTnT in three samples, and that in one of these three samples only a single cTnT peptide was identified, violating commonly used guidelines for the identification of proteins $s^{30}$. However, these guidelines are often focused on intact proteins making the requirement of identifying more than one peptide more feasible than in this specific case, where we are most likely dealing with protein fragments. Considering the high confidence of this match, the quality of the MS/MS spectrum, and the fact that a very abundant cTnT-specific immunoreactivity was also determined, we deemed this match convincing. While it could be possible that the low number of identifications was because of the limit of identification of the assay (Table 1) or because of extensive progressive cTnT degradation (possibly continuing in urine), it cannot be excluded that this effect only occurs in specific pathophysiological conditions. The single selection criterion of our study was a routinely obtained urine sample from a patient with high cTnT concentration in serum (>1000 $\mathrm{ng} / \mathrm{L})$, resulting in a wide range of pathophysiological conditions in the selected patients. In addition to AMI, all three patients where cTnT was identified with LC-MS/MS additionally suffered from proteinuria, possibly causing filtered cTnT fragments to end up in the urine as a result of protein overload of the proximal tubule. 
Previously, Ziebig et al. also detected CTnT in urine of patients suffering from AMI or undergoing heart surgery using the clinical cTnT immunoassay ${ }^{31}$. Patients included in the work of Ziebig also suffered from mild to severe proteinuria, however, their methodology was not intended to be used in urine and was not properly validated for that matrix. This makes it hard to draw firm conclusions, as positive results can be caused by matrix effects or other disturbances. After all, while immunoassays may have relatively low limits of detection, their limit of identification is more questionable. Immunoassays will require multiple epitopes in order to reach the same level of identification certainty as tandem mass spectrometry. In this study, we were able to confirm the findings by Ziebig et al. using the $5^{\text {th }}$ generation cTnT immunoassay (whereas Ziebig used the $2^{\text {nd }}$ generation immunoassay, both by Roche Diagnostics). The thus measured cTnT-specific immunoreactivity was considerable in most patient samples and differed significantly from those measured in healthy controls (figure 3b). However, due to the urinary matrix, the identity of this immunoreactivity is uncertain. Therefore, it is important to interpret figure $3 b$, as well as the study by Ziebig et al. ${ }^{31}$, with caution.

Lastly, a recent study from our group showed that impaired renal clearance is not the main driver behind persistently elevated cardiac troponin concentrations ${ }^{8}$. While the results presented here may seem to be at odds with that conclusion, it is important to consider that the current demonstration of cardiac troponin in urine is limited to subjects suffering from acute myocardial infarction with high cTnT concentrations in serum (>1000 ng/L) and concomitant proteinuria. Involvement of the kidneys in the elimination of cTnT (be it physiological or pathophysiological) does not automatically imply that reduced renal clearance leads to aspecific troponin elevations in the absence of myocardial damage.

In conclusion, we show here, using a targeted mass spectrometry assay, that the highly cardiospecific biomarker cTnT can be present in urine of patients suffering from AMI. Further study to the extent and implications of these observations are required, as well as determining whether this is a non-specific effect caused by concomitant proteinuria, or if this is a more general elimination pathway of cTnT. 


\section{References}

1. Roffi M, Patrono C, Collet JP, et al. 2015 ESC Guidelines for the management of acute coronary syndromes in patients presenting without persistent ST-segment elevation: Task Force for the Management of Acute Coronary Syndromes in Patients Presenting without Persistent ST-Segment Elevation of the European Society of Cardiology (ESC). Eur Heart J 2016;37:267-315.

2. Amsterdam EA, Wenger NK, Brindis RG, et al. 2014 AHA/ACC guideline for the management of patients with nonST-elevation acute coronary syndromes: executive summary: a report of the American College of Cardiology/American Heart Association Task Force on Practice Guidelines. Circulation 2014;130:2354-94.

3. Thygesen K, Alpert JS, Jaffe AS, et al. Third universal definition of myocardial infarction. Journal of the American College of Cardiology 2012;60:1581-98.

4. de Lemos JA. Increasingly sensitive assays for cardiac troponins: a review. Jama 2013;309:2262-9.

5. Twerenbold R, Wildi K, Jaeger C, et al. Optimal Cutoff Levels of More Sensitive Cardiac Troponin Assays for the Early Diagnosis of Myocardial Infarction in Patients With Renal Dysfunction. Circulation 2015;131:2041-50.

6. Cardinaels EP, Altintas S, Versteylen MO, et al. High-Sensitivity Cardiac Troponin Concentrations in Patients with Chest Discomfort: Is It the Heart or the Kidneys As Well? PLoS One 2016;11:e0153300.

7. Fridén V, Starnberg K, Muslimovic A, et al. Clearance of cardiac troponin $T$ with and without kidney function. Clinical biochemistry 2017;50:468-74.

8. van der Linden N, Cornelis T, Kimenai DM, et al. The origin of cardiac troponin T levels in chronic kidney disease. Circulation 2017; accepted.

9. Parmacek MS, Solaro RJ. Biology of the troponin complex in cardiac myocytes. Progress in cardiovascular diseases 2004;47:159-76.

10. Katus HA, Remppis A, Scheffold T, Diederich KW, Kuebler W. Intracellular compartmentation of cardiac troponin $T$ and its release kinetics in patients with reperfused and nonreperfused myocardial infarction. The American journal of cardiology 1991;67:1360-7.

11. Wu AH, Feng YJ, Moore R, et al. Characterization of cardiac troponin subunit release into serum after acute myocardial infarction and comparison of assays for troponin T and I. American Association for Clinical Chemistry Subcommittee on cTnI Standardization. Clinical chemistry 1998;44:1198-208.

12. Cardinaels EP, Mingels AM, van Rooij T, Collinson PO, Prinzen FW, van Dieijen-Visser MP. Time-dependent degradation pattern of cardiac troponin T following myocardial infarction. Clinical chemistry 2013;59:1083-90.

13. Michielsen EC, Diris JH, Kleijnen VW, Wodzig WK, Van Dieijen-Visser MP. Investigation of release and degradation of cardiac troponin T in patients with acute myocardial infarction. Clinical biochemistry 2007;40:851-5.

14. Diris JH, Hackeng CM, Kooman JP, Pinto YM, Hermens WT, van Dieijen-Visser MP. Impaired renal clearance explains elevated troponin T fragments in hemodialysis patients. Circulation 2004;109:23-5.

15. Saritas T, Kuppe C, Moeller MJ. Progress and controversies in unraveling the glomerular filtration mechanism. Curr Opin Nephrol Hypertens 2015;24:208-16.

16. Christensen El, Birn H, Storm T, Weyer K, Nielsen R. Endocytic receptors in the renal proximal tubule. Physiology 2012;27:223-36.

17. Comper WD, Russo LM, Vuchkova J. Are filtered plasma proteins processed in the same way by the kidney? J Theor Biol 2016;410:18-24.

18. Dickson LE, Wagner MC, Sandoval RM, Molitoris BA. The proximal tubule and albuminuria: really! J Am Soc Nephrol 2014;25:443-53.

19. Streng AS, de Boer D, Bouwman FG, et al. Development of a targeted selected ion monitoring assay for the elucidation of protease induced structural changes in cardiac troponin T. Journal of proteomics 2016;136:123-32.

20. Streng AS, de Boer D, Bouwman FG, et al. Validation, optimisation, and application data in support of the development of a targeted selected ion monitoring assay for degraded cardiac troponin T. Data Brief 2016;7:397405 . 
21. Streng AS, De Boer D, Van Doorn WPTM, et al. Identification and characterization of cardiac troponin T fragments in serum of patients suffering from acute myocardial infarction. Clinical chemistry 2017;63:563-72.

22. Michielsen EC, Diris JH, Hackeng CM, Wodzig WK, Van Dieijen-Visser MP. Highly sensitive immunoprecipitation method for extracting and concentrating low-abundance proteins from human serum. Clinical chemistry 2005;51:222-4.

23. Giannitsis E, Kurz K, Hallermayer K, Jarausch J, Jaffe AS, Katus HA. Analytical validation of a high-sensitivity cardiac troponin T assay. Clinical chemistry 2010;56:254-61.

24. Afkarian M, Bhasin M, Dillon ST, et al. Optimizing a proteomics platform for urine biomarker discovery. Molecular \& cellular proteomics : MCP 2010;9:2195-204.

25. Labugger R, Organ L, Collier C, Atar D, Van Eyk JE. Extensive troponin I and T modification detected in serum from patients with acute myocardial infarction. Circulation 2000;102:1221-6.

26. Olszowy P, Buszewski B. Urine sample preparation for proteomic analysis. J Sep Sci 2014;37:2920-8.

27. Thongboonkerd V, McLeish KR, Arthur JM, Klein JB. Proteomic analysis of normal human urinary proteins isolated by acetone precipitation or ultracentrifugation. Kidney Int 2002;62:1461-9.

28. Sigdel TK, Lau K, Schilling J, Sarwal M. Optimizing protein recovery for urinary proteomics, a tool to monitor renal transplantation. Clin Transplant 2008;22:617-23.

29. Court M, Selevsek N, Matondo M, et al. Toward a standardized urine proteome analysis methodology. Proteomics 2011;11:1160-71.

30. Taylor GK, Goodlett DR. Rules governing protein identification by mass spectrometry. Rapid communications in mass spectrometry : RCM 2005;19:3420.

31. Ziebig R, Lun A, Hocher B, et al. Renal elimination of troponin T and troponin I. Clinical chemistry 2003;49:11913. 


\title{
Supplemental information
}

\author{
Supplemental Tables
}

Supplemental Table 1. Characteristics and laboratory values of patients included in the study

\begin{tabular}{|c|c|c|c|c|c|c|}
\hline Pat. No. ${ }^{a}$ & Sex, age & Diagnosis & $\begin{array}{l}\text { cTnT serum } \\
\text { (ng/L) }\end{array}$ & $\begin{array}{l}\text { cTnT urine } \\
\text { (ng/L) }\end{array}$ & $\begin{array}{l}\text { eGFR }^{b} \\
\left(\mathrm{ml} / \mathrm{min} / 1.73 \mathrm{~m}^{2}\right)\end{array}$ & $\begin{array}{l}\text { Urine albuminc } \\
\text { (g/L) }\end{array}$ \\
\hline$a$ & $\mathrm{M}, 80$ & NSTEMI & 2274 & 13000 & 12 & 1 \\
\hline$b$ & $M, 64$ & STEMI & 3494 & 7365 & 13 & 0.3 \\
\hline C & M, 93 & STEMI & 31199 & 125 & 70 & $\mathrm{n} / \mathrm{a}$ \\
\hline 1 & $\mathrm{M}, 71$ & STEMI & 5443 & 27 & 39 & 1 \\
\hline 2 & $F, 90$ & Miscellaneous & 1180 & 7 & 42 & 1 \\
\hline 3 & $M, 82$ & STEMI & 2556 & 355 & 27 & 0.3 \\
\hline 4 & $M, 85$ & NSTEMI & 1220 & 27 & 39 & 0.3 \\
\hline 5 & $F, 70$ & STEMI & 2770 & 15 & 93 & 0.3 \\
\hline 6 & $M, 54$ & Myocarditis & 7891 & 8 & 65 & 0.3 \\
\hline 7 & $M, 52$ & STEMI & 1203 & 38 & 65 & $\mathrm{n} / \mathrm{a}$ \\
\hline 8 & $F, 81$ & STEMI & 1792 & 6 & 58 & Negative \\
\hline 9 & M, 71 & NSTEMI & 1551 & 19 & 21 & Trace \\
\hline 10 & M, 34 & Myocarditis & 8497 & 17 & 112 & Negative \\
\hline 11 & $M, 56$ & Myocarditis & 3803 & 706 & 17 & 0.59 \\
\hline 12 & $M, 77$ & STEMI & 5967 & 17 & 34 & 0.3 \\
\hline 13 & $F, 91$ & STEMI & 2681 & 14 & 25 & $\mathrm{n} / \mathrm{a}$ \\
\hline 14 & $F, 84$ & NSTEMI & 3783 & 50 & 17 & Trace \\
\hline 15 & $M, 65$ & Myocarditis & 1225 & $<3.00$ & 82 & Negative \\
\hline 16 & $F, 55$ & Cardiac arrest & 1715 & 5 & 80 & 0.3 \\
\hline 17 & M, 25 & Myocarditis & 1688 & 12 & 122 & 0.3 \\
\hline
\end{tabular}

a Patient numbers a-c belong to patients with MS/MS identification of cTnT and correspond to the numbers in table 2 and the figures in the main article. Numbers 1-17 belong to patients without MS/MS identifications.

${ }^{b}$ Estimated glomerular filtration rate (eGFR) was calculated by the CKD-EPI formula as proposed by Levey et al., Ann Intern Med 2009; 150(9):604-612., as follows: eGFR = $141 \times \min (\mathrm{Scr} / \mathrm{k}, 1)^{\alpha} \times \max (\mathrm{Scr} / \mathrm{k}, 1)^{-1.209} \times 0.993^{\text {Age }} \times 1.018$ [if female] $\times 1.159$ [if black], where Scr is serum creatinine, $\mathrm{k}$ is 0.7 for females and 0.9 for males, $\alpha$ is -0.329 for females and -0.411 for males, min indicates the minimum of Scr/K or 1, and max indicates the maximum of Scr/k or 1.

c Urine albumin levels were used as indicators of proteinuria. $\mathrm{n} / \mathrm{a}$ indicates that albumin levels were not available, negative indicates albumin levels $<0.05 \mathrm{~g} / \mathrm{L}$, and trace means an albumin level between 0.05 and $0.1 \mathrm{~g} / \mathrm{L}$. 
Cardiac troponins: State of the (He)Art 


\section{Large variation in measured cardiac}

troponin T concentrations after standard addition in serum or plasma of different individuals

Noreen van der Linden*, Sander Streng*, Otto Bekers, Will Wodzig, Steven Meex, Douwe de Boer - Clin Chem. 2017 Jul;63(7):1300-1302 


\section{Introduction}

During acute myocardial infarction (AMI), cardiac troponin $\mathrm{T}(\mathrm{cTnT})$ is released from the damaged myocardium. One would expect a strong correlation between concentrations of cTnT measured in the blood after AMI and the extent of myocardial damage. However, various studies have shown a rather moderate correlation between infarct size and cTnT concentrations $^{1,2}$. Frequently considered explanations for this phenomenon are methodological, or focused on physiological factors associated with differences in release and elimination of $\mathrm{CTnT}^{2}$. In contrast, less attention has been paid to the possible presence of inherent factors in the blood, such as (auto)antibodies or proteases, that might modify CTnT or interfere with the assay. We hypothesized that these factors may be ubiquitously present in the general population, and might affect the measured concentrations of cTnT in individuals.

\section{Methods}

We measured $c T n T$, cardiac troponin I (cTnl), creatine kinase MB isoenzyme (CK-MB) and myoglobin concentrations in sera from 24 healthy volunteers (age range 22 - 54 years) before and after the addition of serum from a patient suffering from AMI (age 59 years, cTnT $\approx 19.5 \mu \mathrm{g} / \mathrm{L}$ ). All participants gave written informed consent and leftover material was used in accordance with the code of proper secondary use of human tissue in the Netherlands. Spiking of AMl-patient serum ( $40 \mu \mathrm{L}$ in $760 \mu \mathrm{L}$ of volunteer serum) was performed in triplicate. cTnT (hs-cTnT: limit of detection (LoD) $5 \mathrm{ng} / \mathrm{L} ; 10 \%-C V$ at $13 \mathrm{ng} / \mathrm{L}$ ), CK-MB (LoD of $0.3 \mu \mathrm{g} / \mathrm{L}, 20 \%-C V$ at $1 \mu \mathrm{g} / \mathrm{L}$ ), and myoglobin (LoD $21 \mu \mathrm{g} / \mathrm{L}$ ) were determined on the COBAS (Roche Diagnostics), and cTnl (hs-cTnl: LoD 1.1-1.9 ng/L, 10\%-CV at $4.7 \mathrm{ng} / \mathrm{L}$ ) on the Architect (Abbott Diagnostics) (information based on product inserts). Biomarker concentrations were corrected for their baseline concentrations, and outliers (Grubbs test) were excluded. To make the results of different biomarkers easily comparable, the subject-specific deviation was calculated. The subject-specific deviation was defined as the percentage of deviation of a measured biomarker concentration in an individual subject compared to the mean 
concentration of that biomarker across all samples after spiking of a fixed amount of material.

\section{Results}

The subject-specific deviation of cTnT after spiking varied between $-54 \%$ and $+22 \%$ (Fig. 1) . Next, the inter-subject coefficient of variation $\left(\mathrm{CV}_{\mathrm{g}}\right)$ was calculated for $\mathrm{CTnT}(14.6 \%)$, which was significantly higher than the $\mathrm{CV}_{\mathrm{g}}$ for $\mathrm{CTnl}$ (6.9\%), CK-MB (2.6\%), and myoglobin (3.0\%) (all $p<0.001)$ measured in the same samples. In contrast, no significant difference was shown in the coefficient of variation between the triplicate measurements of the different cardiac biomarkers ( $\pm 1.5 \%$, not shown). Interestingly, when this experiment was repeated in serum from the same individuals that was deproteinated by perchloric acid and neutralized to $\mathrm{pH}$ 7.4, the $\mathrm{CV}_{\mathrm{g}}$ for $\mathrm{CTnT}$ concentration significantly decreased from $14.6 \%$ to $6.5 \%(\mathrm{p}<0.001)$. These findings were confirmed in an independent duplicate experiment using purified human cTnT (cat\#8T13, HyTest LTD, Turku, Finland) instead of AMI-patient serum.

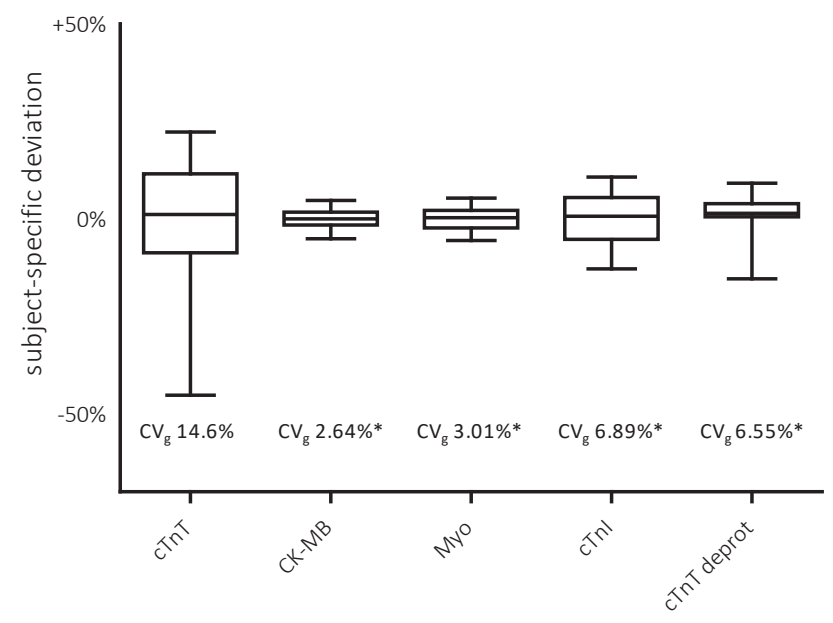

Figure 1. Subject-specific deviation (\%) of cardiac biomarkers added to serum samples of healthy individuals. Boxplots showing quartiles and range (min-max) of the subject-specific deviations for cardiac biomarkers in 24 healthy volunteers (mean of triplicates). We detected two outliers (one for CTnT in deproteinated serum and one for CK$\mathrm{MB})$ within triplicate measurements and excluded these single measurements. ${ }^{*}$ Significant $(p<0.001)$ difference in the inter-subject coefficient of variation of a biomarker compared to that of cTnT in unprocessed serum samples. CK-MB, creatine kinase MB isoenzyme; cTnl, cardiac troponin I; cTnT, cardiac troponin $\mathrm{T}$; $\mathrm{CV}_{\mathrm{g}}$, inter-subject coefficient of variation; deprot, deproteinated serum; Myo, myoglobin. 
The wide inter-subject variation in cTnT concentrations observed in these first experiments was validated in a consecutive experiment in which $2.5 \mu \mathrm{L}$ of purified human cTnT (HyTest) was spiked in leftover heparin plasma samples (250 $\mu \mathrm{L})$ from 119 patients. Again, concentrations were corrected for baseline values. In this population, we observed a subjectspecific deviation between $-39 \%$ and $+37 \%$ that was normally distributed (D'AgostinoPearson omnibus test, $\mathrm{p}=0.210$ ). The $\mathrm{CV}_{\mathrm{g}}$ of $15.8 \%$ in this experiment was comparable to that in our previous experiment.

\section{Conclusion}

Thus, we showed a substantial and specific variation in CTnT concentrations after the standard addition of equal amounts of cTnT to both serum and plasma of several individuals. This inter-subject variation decreased significantly when serum was deproteinated. Currently, we can only speculate about the nature of the observed variations. Since the effect diminished after the removal of proteins from serum, a protein factor could be responsible. Individual differences in proteolytic degradation of cTnT cannot be excluded as the responsible factor, but seems to be unlikely since the cTnT present in the serum sample used for spiking will already be fragmented and the observed pattern remains similar when purified human cTnT was used ${ }^{3}$. Also, the involvement of heterophilic antibodies, like human anti-mouse antibodies (HAMA) and rheumatoid factor, is unlikely since the variation is ubiquitously present. A remaining possible explanation could be the blocking of epitopes or alternative folding of cTnT due to the interaction with specific (auto)antibodies ${ }^{4}$. More research is needed to identify the exact factor(s) responsible for the observed variation, including their mechanism of action, to ascertain whether or not these differences also occur with non-foreign cTnT in vivo, and to determine the exact clinical implications including the determination of infarct size. 


\section{References}

1. Reinstadler SJ, Feistritzer HJ, Klug G, et al. High-sensitivity troponin T for prediction of left ventricular function and infarct size one year following ST-elevation myocardial infarction. Int J Cardiol 2016;202:188-93.

2. Cobbaert $\mathrm{CM}$, Bootsma M, Boden $\mathrm{H}$, et al. Confounding factors in the relation between high sensitivity cardiac troponin $\mathrm{T}$ levels in serum and infarct size of patients with first ST-elevation myocardial infarction. Int J Cardiol 2014;172:e3-5.

3. Cardinaels EP, Mingels AM, van Rooij T, Collinson PO, Prinzen FW, van Dieijen-Visser MP. Time-dependent degradation pattern of cardiac troponin T following myocardial infarction. Clin Chem 2013;59:1083-90.

4. Adamczyk M, Brashear RJ, Mattingly PG. Prevalence of autoantibodies to cardiac troponin $T$ in healthy blood donors. Clin Chem 2009;55:1592-3. 
Cardiac Troponins: State of the (He)Art 


\section{Combining cardiac troponin l and cardiac}

\section{troponin $T$ in the early diagnosis of acute}

\section{myocardial infarction}

Noreen van der Linden*, Karin Wildi, Raphael Twerenbold, John Pickering, Martin Than, Louise Cullen, Jaimi Greenslade, William Parsonage, Thomas Nestelberger, Jasper Boeddinghaus, Patrick Badertscher, Maria Rubini Giménez, Lieke Klinkenberg, Otto Bekers, Aline Schöni, Dagmar Keller, Zaid Sabti, Christian Puelacher, Janosch Cupa, Lukas Schumacher, Nikola Kozhuharov, Karin Grimm, Samyut Shrestha, Dayana Flores Widmer, Michael Freese, Claudia Stelzig, Ivo Strebel, Oscar Miró, Katharina Rentsch, Beata Morawiec, Damian Kawecki, Wanda Kloos, Jens Lohrmann, Mark Richards, RichardTroughton, Christopher Pemberton, Stefan Osswald, Marja van Dieijen-Visser, Alma Mingels, Tobias Reichlin, Steven Meex, Christian Mueller 


\section{Abstract}

\section{Background}

Combining two signals of cardiomyocyte damage, cardiac troponin I (cTnl) and T (cTnT), might overcome some individual pathophysiological and analytical limitations and thereby increase diagnostic accuracy for acute myocardial infarction (AMI) with a single blood draw. We aimed to evaluate the diagnostic performance of combinations of cTnl and cTnT for the early diagnosis of AMI.

\section{Methods}

The diagnostic performance of combining cTnl and CTnT (simple mathematical derivations and a combination algorithm) was evaluated in a large prospective multicenter diagnostic study of patients with suspected AMI. The optimal thresholds were externally validated in a second large multicenter diagnostic study.

\section{Results}

Combining cTnl and cTnT did not consistently increase overall diagnostic accuracy as compared to the individual isoforms. In contrast, the combination seemed to improve the very early rule-out, which at the guideline-recommended cut-offs is limited (6-11\%) and assay dependent. Application of optimized cut-off values using the sum ( $9 \mathrm{ng} / \mathrm{L})$ and the product $\left(18 \mathrm{ng}^{2} / \mathrm{L}^{2}\right)$ of $\mathrm{cTnl}$ and $\mathrm{CTnT}$ led to a significant increase in rule-out to $34-41 \%$ in the original (sum: negative predictive value (NPV) 100\% (95\%Cl: 99.5 100\%); product: NPV 100\% (95\% Cl: 99.5-100\%) and in the external validation cohort (sum: NPV 99.6\% (95\%Cl: 99.0-99.9\%); product: NPV 99.4\% (95\% Cl: 98.8-99.8\%) as in comparison to the ESC 2015 algorithm. The use of a combination algorithm (cTnl $<4 \mathrm{ng} / \mathrm{L}$ and $\mathrm{cTnT}<9 \mathrm{ng} / \mathrm{L}$ ) showed comparable results (rule out: 40-43\%; NPV original cohort 99.9\% (95\%Cl: 99.2-100\%); NPV external validation cohort 99.5\% (95\%Cl: 98.9-99.8\%)).

\section{Conclusions}

New strategies combining cTnl and CTnT measurements may significantly increase the number of patients eligible for very early and safe rule-out, but do not seem helpful for the rule-in of AMI. 


\section{Introduction}

Approximately $10 \%$ of all patients seeking medical attention at the emergency department (ED) report chest discomfort, a complaint that reflects many potential etiologies including acute myocardial infarction (AMI) ${ }^{1}$. Rapid identification of patients with $\mathrm{AMI}$ is of profound clinical importance for fast initiation of medical treatment and management ${ }^{2}$. In addition, rapid rule-out of patients without $\mathrm{AMI}$ can overcome prolonged patient anxiety, unnecessary resource use and overcrowding in the $\mathrm{ED}^{3-9}$. Despite major improvements in diagnostic accuracy due to the introduction of high-sensitivity cardiac troponin (hs-cTn) assays and data-driven optimized diagnostic algorithms, rapid, accurate and safe rule-out based on a single measurement of hs-cTn is still possible only in a minority of patients ${ }^{2,3,10}$.

Current guidelines recommend to measure one of the cardiac specific isoforms of the cardiac troponin (cTn) complex: $\mathrm{cTnl}$ or $\mathrm{cTnT}^{2,11,12}$. Despite differences in biochemical characteristics and release kinetics ${ }^{13,14}$, a recent direct comparison between high-sensitivity cTnl (hs-cTnl) and T (hs-cTnT) showed similar, high diagnostic accuracy for AMl emphasizing the similarities between both isoforms ${ }^{15}$. Based on the observation of an imperfect correlation between blood concentrations of cTnT and cTnl in chronic and acute disorders ${ }^{16,17}$, and in analogy to the quantification of renal function using creatinine and cystatin $\mathrm{C}$, where the combination of two parameters associated with the same pathophysiological process but influenced by distinct factors lead to a more precise and accurate indicator ${ }^{18}$, we hypothesize that combining cTnl and cTnT will overcome independent pathophysiological, pre-analytical and analytical differences of the individual molecules, and might therefore have higher diagnostic accuracy for AMI than either cTnl or cTnT. This hypothesis was tested in two large prospective multicenter diagnostic studies. 


\section{Methods}

\section{Patients and setting}

The combination of cTnl and CTnT for the diagnosis of AMI was investigated in two studies; The primary cohort was the Advantageous Predictors of Acute Coronary Syndrome Evaluation (APACE) study 3,15,19,20, and the secondary (external validation) cohort was the 2Hour Accelerated Diagnostic Protocol to Assess Patients Witch Chest Pain Symptoms Using Contemporary Troponins as the Only Biomarker (ADAPT) study ${ }^{21}$.

APACE is an ongoing prospective international multicenter diagnostic study that enrolls patients presenting to the ED with acute chest discomfort with an onset of peak within the last 12 hours. Patients are enrolled regardless of their renal function. Only patients with terminal kidney failure on chronic dialysis are excluded. This analysis contains data of patients enrolled between April 2006 and May 2013 who had a final diagnosis adjudicated by two independent cardiologists ( $n=3029$ ). For this analysis, patients were excluded if hs-cTnl or hsCTnT blood concentrations at presentation were not available $(n=661)$, if the final adjudicated diagnosis was ST-elevation myocardial infarction (STEMI) $(n=74)$, or if the final diagnosis remained unclear after adjudication and at least one cTn level was elevated (possibly indicating the presence of AMI) ( $n=69)$.

In the ADAPT cohort (4 cohorts combined: ADAPT-Brisbane, ADAPT-Christchurch, EDACS$R C T, A D A P T-R C T)$, patients with at least 5 min of symptoms consistent with acute coronary syndrome ${ }^{22}$, but without ST-segment elevation, were enrolled at two EDs in Brisbane, Australia and Christchurch, New Zealand between November 2007 and February 2011.

Both studies were carried out according to the principles of the Declaration of Helsinki, approved by the local ethics committees, and registered at clinicaltrial.gov (APACE: NCT00470587) or at the Australia-New Zealand Clinical Trials Registry (ADAPT: ACTRN12611001069943). Written informed consent was obtained from all patients.

\section{Routine clinical assessment}

In both cohorts, patients underwent routine clinical assessment that included medical history, physical examination, standard blood tests including serial measurements of local 
(hs)-cTn, 12-lead ECG, chest radiography, continuous ECG rhythm monitoring and pulse oximetry. Management of patients was left to the discretion of the attending physician.

\section{Adjudicated final diagnosis}

In the APACE cohort, adjudication of the final diagnosis was performed by two independent cardiologists at the core laboratory (University Hospital Basel) applying the universal definition of $\mathrm{AMI}^{23}$ using two sets of data: first, all available medical records obtained during clinical care including history, physical examination, results of laboratory testing (including serial clinical (hs)-cTn levels, radiologic testing, ECG, echocardiography, cardiac exercise test, lesion severity and morphology in coronary angiography - pertaining to the patient from the time of ED presentation to 90-day follow up; second, study-specific assessments including detailed chest pain characteristics using 34 predefined criteria, serial hs-cTnT blood concentrations obtained from study samples, and clinical follow-up by telephone and/or mail. In situations of disagreement about the diagnosis, cases were reviewed and adjudicated in conjunction with a third cardiologist. These procedures were comparable to those in the ADAPT cohort, where the adjudication of the final diagnosis was performed by two independent cardiologists blind to results of the index-test biomarkers under investigation, but with knowledge of the clinical record, ECG, and serial cTnl results from routine care (details of adjudication are given in the Supplementary Data).

In both cohorts, AMI was defined and (hs-)cTn interpreted as recommended in the current guidelines ${ }^{2,24,25}$. In brief, AMI was diagnosed when there was evidence of myocardial necrosis in association with a clinical setting consistent with myocardial ischemia. Myocardial necrosis was diagnosed by at least one cTn value above the $99^{\text {th }}$ percentile (or for the conventional cTn assays above the $10 \%$ imprecision value if not fulfilled at the $99^{\text {th }}$ percentile) together with a significant rise and/or fall. The criteria used to define a rise and/or fall in conventional cTn and hs-cTnT are described in detail in the method section in the data supplement. All other patients were classified in the categories of unstable angina (UA), Non Cardiac Chest Pain (NCCP), cardiac but non-coronary disease (e.g. tachyarrhythmias, perimyocarditis), and symptoms of unknown origin with normal levels of hs-cTnT. 


\section{Measurement of hs-cTnT and hs-cTnl}

After centrifugation, serum was frozen at $-80^{\circ} \mathrm{C}$ until measurement with hs-cTn assays. HscTnl was measured by using the ARCHITECT High Sensitive STAT Troponin I assay (Abbott Laboratories). According to the manufacturer, the 99th percentile concentration is $26.2 \mathrm{ng} / \mathrm{L}$ with a corresponding coefficient of variation (CV) of $<5 \%{ }^{26}$. Hs-cTnT was measured with the Roche hs-cTnT assay. The 99th percentile among healthy subjects is $14 \mathrm{ng} / \mathrm{L}$, with a $10 \%$ analytical variation at $13 \mathrm{ng} / \mathrm{L}^{27}$. Data presented here were not affected by the 2010-2012 hs-cTnT low-end shift in APACE and appropriately corrected in ADAPT ${ }^{28-30}$. Calculation of the glomerular filtration rate (eGFR) was performed using the abbreviated Modification of Diet in Renal Disease (MDRD) formula ${ }^{31}$.

\section{Statistical analysis}

We evaluated the diagnostic accuracy and performance of the combined hs-cTnl and hs-cTnT measurement in two different ways: First, we examined simple clinically easily interpretable mathematical derivations (sum, product, ratio). Second, we tested a combination algorithm of hs-cTnl and hs-cTnT. Data are expressed as median \pm interquartile range (IQR) for continuous variables and as numbers ( $n$ ) and percentages (\%) for categorical variables. Continuous variables were compared with the Mann-Whitney $U$ test, and categorical variables were compared by use of the Pearson $\chi^{2}$ test. Cohen's kappa statistic was performed to examine the agreement between rule-in and rule-out at presentation based on hs-cTnT and hs-cTnl according to the two diagnostic algorithms recommended with a class I recommendation in the current European Society of Cardiology (ESC) guidelines: the 0/3hhs-cTn-algorithm and the Oh/1h-hs-cTn-algorithm². Mathematical derivations were calculated from raw data after the correction of undetectable low concentrations into $0.1 \mathrm{ng} / \mathrm{L}$. Binary logistic regression analyses were used to calculate predicted probabilities for combined test variables.

Receiver-operating characteristics (ROC) curves were constructed to assess diagnostic performance at presentation and $1 \mathrm{~h}$ after initial presentation including the absolute change value. Diagnostic accuracy was reported as the area under the ROC curve (AUC) and the 
corresponding 95\% confidence intervals $(95 \% \mathrm{Cl})$. The comparison of dependent and independent AUCs was performed as recommended by Hanley and McNeil ${ }^{32,33}$. Oh and Oh/1h serial sampled hs-cTn blood concentrations were combined to represent the current gold standard of clinical care as suggested in the 2015 ESC guidelines ${ }^{2}$. Furthermore, integrated discrimination improvement (IDI) and category-free net reclassification improvement (cfNRI) were calculated.

For the determination of optimal cut-off values for mathematical derivations (minimal negative predictive value (NPV) of $99.6 \%$ and a positive predictive value (PPV) of $75.0 \%$, respectively, to match the performance of the $0 \mathrm{~h} / 1 \mathrm{~h}$-hs-cTn-algorithm ${ }^{2,34-36}$, the cohort was randomly divided in a derivation ( $80 \%$ of patients) and a validation sub-cohort (20\% of patients).

For the cut-off values in the combination algorithm, we determined the optimal combination of hs-cTn thresholds (maximized specificity and a minimum sensitivity of $99 \%$ ) based on a smoothed average of 500 bootstraps of the original cohort, in which we varied the hs-cTn threshold for each troponin assay in steps of $0.1 \mathrm{ng} / \mathrm{L}$.

All hypothesis testing was two-tailed, and values of $p<0.05$ were considered statistically significant. We did not adjust for multiple testing. Statistical analyses were performed with SPSS for Windows 23.0 (SPSS Inc), MedCalc 9.6.4.0 (MedCalc software) and R version 3.2.4 (with packages 'boot' v1.3-18 and 'fields' v8.10). 


\section{Results}

\section{Distribution of hs-cTn concentrations at presentation in patients with suspected AMI}

Baseline characteristics of 2225 patients in the APACE cohort presenting to the ED with suspected AMI are shown in Supplementary Table 1. The adjudicated final diagnosis was AMI (NSTEMI) in $18 \%$ of patients (85\% had type I and 15\% type II AMI), UA in 10\%, cardiac but not coronary artery disease in $14 \%$, NCCP in $54 \%$, and symptoms of unknown origin in $5 \%$. AMI patients had significant higher levels of hs-cTnl and hs-cTnT at presentation compared with the no-AMI group (hs-cTnl median $115.2 \mathrm{ng} / \mathrm{L}$ (IQR: 21.7-632.9) vs. $3.5 \mathrm{ng} / \mathrm{L}$ (IQR: 2.2-7.2) $\mathrm{P}<0.001$; hs-cTnT median $64.1 \mathrm{ng} / \mathrm{L}$ (IQR: 28.0-152.4) vs. $7.0 \mathrm{ng} / \mathrm{L}$ (IQR: 4.0-12.4) P<0.001; Supplementary Table 3 and Supplementary Figure 1). The correlation between hs-cTnl and hs-cTnT concentrations at presentation was moderate (Figure 1).

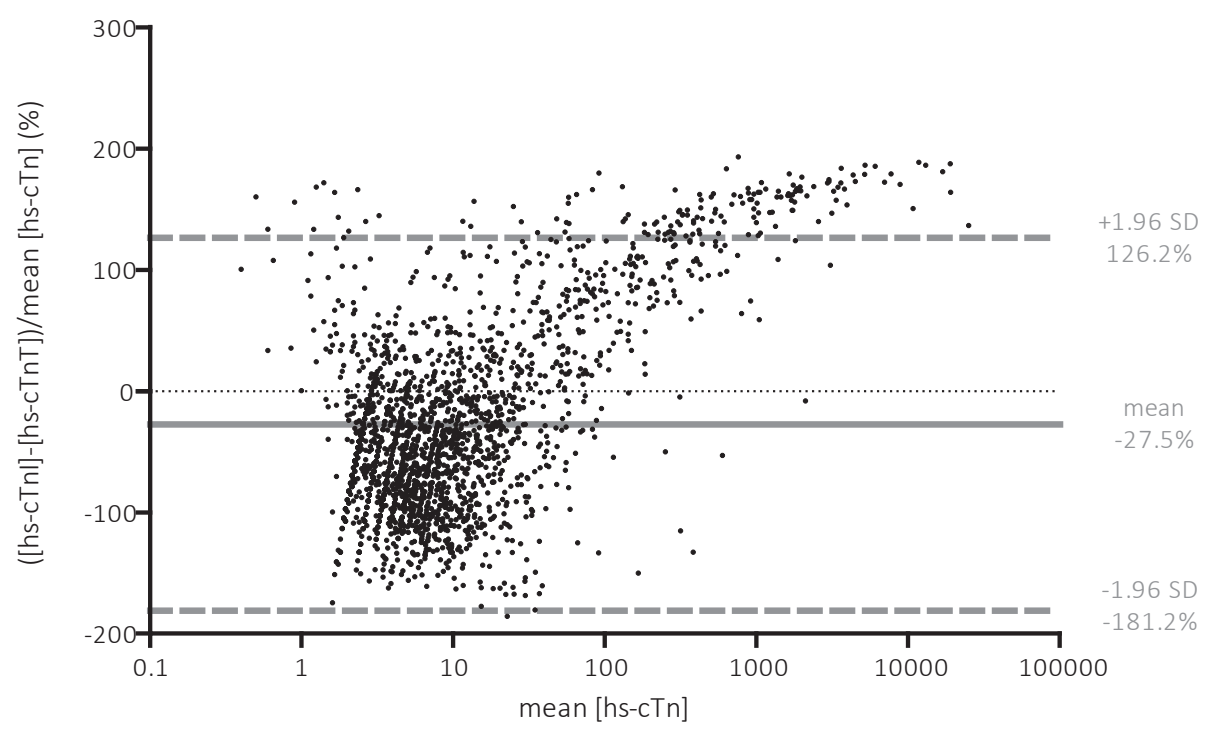

Figure 1. Bland Altman plot of hs-cTnT and hs-cTnl at presentation in the APACE cohort. Bland Altman plot displaying hs-cTnl and hs-cTnT concentrations at presentation in the APACE cohort ( $n=2225)$. 


\section{Application of the ESC Oh/3h-algorithm}

AMI could be ruled-out in 157 (7.1\%, sensitivity 99.2\%, specificity 8.4\%, NPV 98.1\%) and 133 (6.0\%, sensitivity $99.7 \%$, specificity $7.2 \%$, NPV 99.2\%) patients with a single blood draw at presentation, using hs-cTnl or hs-cTnT, respectively. The agreement on patient allocation between hs-cTnl and hs-cTnT for rule-out at presentation was good ( $\mathrm{k}=0.90$ ) (Figure 3, Supplementary Table 4a).

\section{Application of the ESC Oh/1h-algorithm}

AMI could be ruled-out in 149 (6.7\%, sensitivity 100\%, specificity 8,1\%, NPV 100\%) and 235 (10.6\%, sensitivity $100 \%$, specificity $12.9 \%$, NPV 100\%) patients after a single blood draw at presentation, using hs-cTnl or hs-cTnT, respectively. Direct rule-in could be achieved in 331 (14.9\%, sensitivity 62.8\%, specificity 95.6\%, PPV 75.5\%) and 273 (12.3\%, sensitivity 57.8\%, specificity 2.4\%, PPV 84.2\%) subjects, using hs-cTnl or hs-cTnT, respectively. The agreement on patient allocation at presentation between hs-cTnl and hs-cTnT was moderate for ruleout ( $\mathrm{k}=0.42)$ and good for rule-in ( $\mathrm{k}=0.79)$ (Figure 3, Supplementary Tables $4 \mathrm{~b}$ and $4 \mathrm{c}$ ).

\section{Diagnostic performance of combined hs-cTnl and hs-cTnT measurement at presentation}

The diagnostic accuracy in the APACE cohort, as quantified by AUC was evidently lower for ratio than for the other combined measurements and individual isoforms (Table 1 and Figure 2). Addition of a second isoform to Oh hs-cTn led to numerically small, but statistically significant improvements in diagnostic accuracy of hs-cTnT, but not hs-cTnl. Furthermore, addition of a combined measurement at presentation to the $0 \mathrm{~h}$ and $0 \mathrm{~h} / \mathrm{h}$ change concentrations led to a numerically small, but statistically significant improvement in diagnostic accuracy of hs-cTnl, but not of hs-cTnT (Supplementary Table 5). Reclassification statistics (IDI and cf-NRI) did not uniformly show incremental value of combining cardiac troponins at presentation when applied to the entire APACE cohort (Supplementary Tables $6 a$ and $6 b)$. 
Table 1. Diagnostic accuracy of hs-cTnl, hs-cTnT, the combination and athematical derivations their (sum, product and ratio) for the diagnosis of $\mathrm{AMI}$ at presentation.

\begin{tabular}{llll}
\hline Parameters & AUC $(95 \% \mathrm{Cl})$ & $\begin{array}{l}\text { Compared with hs-cTnl } \\
(\mathrm{p} \text {-value })\end{array}$ & $\begin{array}{l}\text { Compared with hs-cTnT } \\
\text { (p-value) }\end{array}$ \\
\hline hs-cTnl & 0.93 & 0.714 \\
& $(0.92-0.94)$ & \\
hs-cTnT & 0.93 & 0.714 & \\
& $(0.92-0.94)$ & & 0.002 \\
hs-cTnl \& hs-cTnT & 0.93 & 0.789 & 0.114 \\
Sum & $(0.92-0.94)$ & & \\
Product & 0.94 & 0.053 & 0.078 \\
Ratio & $(0.93-0.95)$ & & $<0.001$ \\
& 0.94 & 0.007 & \\
\hline
\end{tabular}

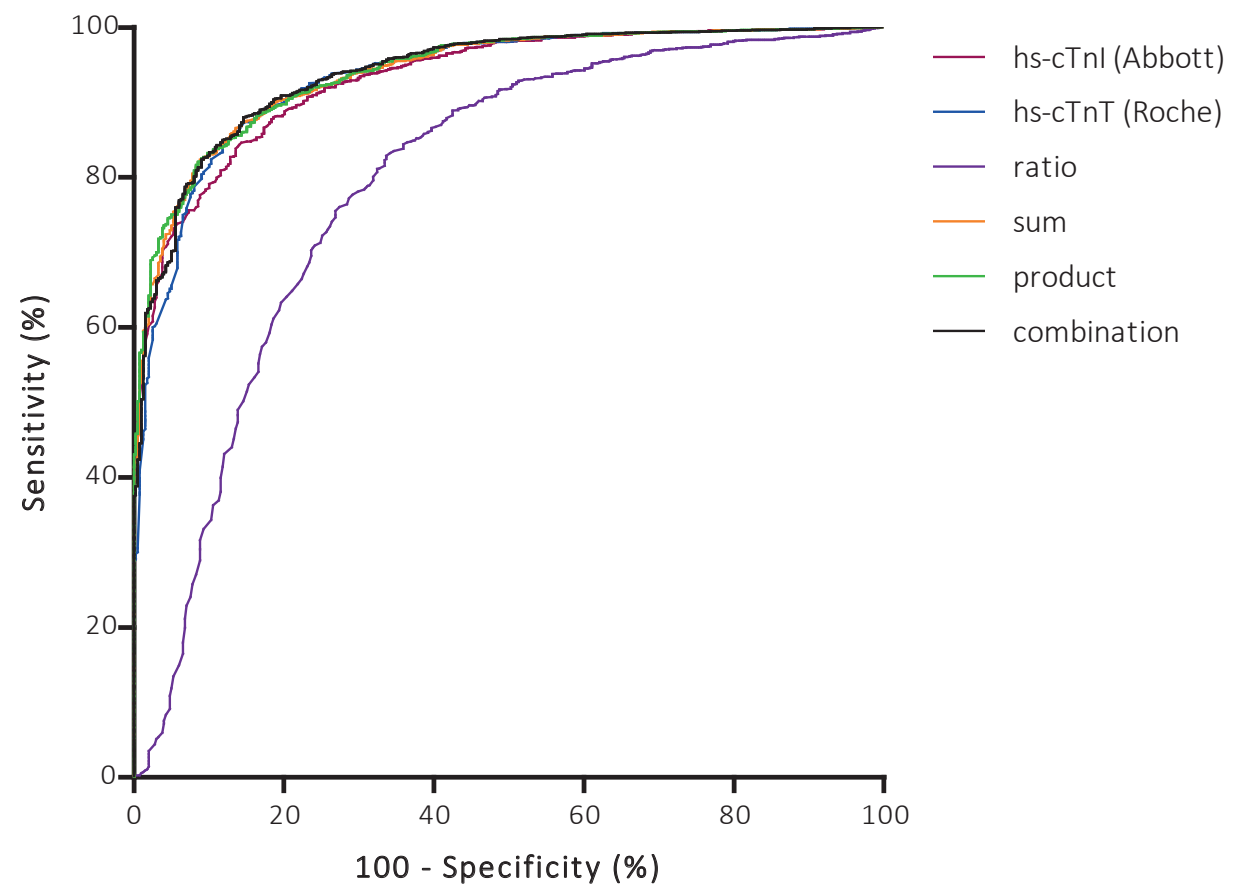

Figure 2. ROC curves of the diagnostic performance of high-sensitivity cTn and their ratio, sum and product for NSTEMI in the APACE cohort. Diagnostic performance of high-sensitive cTn for non-ST segment myocardial infarction at presentation to the emergency department with acute chest pain. Receiver-operating-characteristic curves show the diagnostic accuracy of high-sensitive cardiac troponins I and $\mathrm{T}$, their ratio, sum and product. 
Table 2a. Performance of cut-off values for rule-out and rule-in of sum and product at presentation in the original validation cohort ( $n=2225 ; 398$ AMI, 1827 NO AMI).

\begin{tabular}{|c|c|c|c|}
\hline Rule-out & Sum $<9 \mathrm{ng} / \mathrm{L}$ & Product $<18 \mathrm{ng}^{2} / \mathrm{L}^{2}$ & hs-cTnl $<4 \mathrm{ng} / \mathrm{L}$ AND hs-cTnT $<9 \mathrm{ng} / \mathrm{L}$ \\
\hline AMI & $0(0.0 \%)$ & $0(0.0 \%)$ & $1(0.3 \%)$ \\
\hline No AMI & $746(40.8 \%)$ & $782(42.8 \%)$ & $885(48.4 \%)$ \\
\hline NPV & $100 \%(99.5 \%-100 \%)$ & $100 \%(99.5 \%-100 \%)$ & $99.9 \%(99.2 \%-100 \%)$ \\
\hline Rule-in & Sum $>99 \mathrm{ng} / \mathrm{L}$ & Product $>1608 \mathrm{ng}^{2} / \mathrm{L}^{2}$ & \\
\hline AMI & $249(62.2 \%)$ & $261(65.6 \%)$ & \\
\hline No AMI & 75 (4.1\%) & $79(4.3 \%)$ & \\
\hline$P P V$ & $76.9 \%(71.8 \%-81.3 \%)$ & $76.8 \%(71.9 \%-81.2 \%)$ & \\
\hline
\end{tabular}

Table $2 \mathrm{~b}$. Performance of cut-off values for rule-out and rule-in of sum and product at presentation in the external validation cohort ( $n=2537 ; 408$ AMI, 2129 NO AMI).

\begin{tabular}{|c|c|c|c|}
\hline Rule-out & Sum $<9 \mathrm{ng} / \mathrm{L}$ & Product $<18 \mathrm{ng}^{2} / \mathrm{L}^{2}$ & hs-cTnl $<4$ ng/L AND hs-cTnT $<9$ ng/L \\
\hline AMI & $4(1.0 \%)$ & $6(1.5 \%)$ & $5(1.2 \%)$ \\
\hline No AMI & $984(46.2 \%)$ & 1041 (48.9\%) & $1083(50.9 \%)$ \\
\hline NPV & $99.6 \%(99.0 \%-99.9 \%)$ & $99.4 \%(98.8 \%-99.8 \%)$ & $99.5 \%(98.9 \%-99.8 \%)$ \\
\hline Rule-in & Sum > $99 \mathrm{ng} / \mathrm{L}$ & Product $>1608 \mathrm{ng}^{2} / \mathrm{L}^{2}$ & \\
\hline AMI & $273(66.9 \%)$ & $294(72.1 \%)$ & \\
\hline No AMI & $39(1.8 \%)$ & $43(2.0 \%)$ & \\
\hline$P P V$ & $87.5 \%(83.3 \%-91.0 \%)$ & $87.2 \%(83.2 \%-90.6 \%)$ & \\
\hline
\end{tabular}

\section{Early allocation based on simple mathematical derivations of hs-cTnl and hs-cTnT}

We examined the use of simple mathematical derivations, sum and product, on the allocation of patients at presentation. In a randomly selected derivation cohort of 1799 patients (313 AMI, 1486 no AMI), thresholds for rule-out and rule-in achieving a NPV of at least $99.6 \%$ and a PPV of 75.0\%, respectively, were: rule-out cut-off for the sum of $9 \mathrm{ng} / \mathrm{L}$ and for the product of $18 \mathrm{ng}^{2} / \mathrm{L}^{2}$ (NPV both $100 \%$ (95\% Cl, 99.4-100\%), and a rule-in cut-off for the sum of $99 \mathrm{ng} / \mathrm{L}$ and for the product of $1608 \mathrm{ng}^{2} / \mathrm{L}^{2}$ (PPV sum $75.1 \%$ (95\% Cl 69.3\% 80.3\%), PPV product 75.1\% (95\% Cl 69.5\%-80.1\%)). When these cut-off values were applied to the internal validation cohort of 426 patients ( $85 \mathrm{AMI}, 341$ no $\mathrm{AMI}$ ), we found comparable results for sum (rule-out: sensitivity 100\%, specificity 41.3\%, NPV 100\%; rule-in: sensitivity 65.9\%, specificity 96.8\%, PPV 83.6\%) and product (rule-out: sensitivity 100\%, specificity 42.2\% NPV 100\%; rule-in: sensitivity 65.9\%, specificity 96.8\%, PPV 83.6\%). Application of 
these cut-off values in the original cohort (APACE) would cause a 3-to-5-fold increase in the number of rule-outs at presentation (Table 2a, Figure 3) as compared to the 2015 ESC algorithms. Applying these cut-off values in the external validation cohort (ADAPT) (patient characteristics are shown in Supplementary Table 2), would lead to an early rule- out of more than 45\% in subjects without AMI (NPV sum: 99.6\%; NPV product: 99.4\%; Table 2b).

\section{Early rule-out based on a combination algorithm consisting of hs-cTnl and hs-cTnT}

Examining rule-out based on a combination algorithm, an optimal cut-off value (NPV 99.6\%) was found for the combination of hs-cTnT $<9.8 \mathrm{ng} / \mathrm{L}$ and hs-cTnl $<4.8 \mathrm{ng} / \mathrm{L}$. From a pragmatic point of view, we rounded these cut-off values down to hs-cTnT $<9 \mathrm{ng} / \mathrm{L}$ and hs-cTnl $<4 \mathrm{ng} / \mathrm{L}$. Applying this combination algorithm in the original cohort (APACE) would lead to a 4-to-6fold increase in the number of rule-outs at presentation (Table 2a, Figure 3) (sensitivity 99.7\%, specificity 48.8\%, NPV 99.9\%). Application of this algorithm in the external validation cohort (ADAPT), enables early rule-out in $>50 \%$ of subjects without AMI (sensitivity $98.8 \%$, specificity 50.9\%, NPV 99.5\%) (Table 2b).

\section{Discussion}

We evaluated the combination of cTnl and CTnT for the early diagnosis of AMI in two large prospective diagnostic multicenter studies, and report three major findings.

First, the number of direct rule-outs at presentation using the algorithms of the current ESC guidelines ${ }^{2}$ is limited (7-13\% of subjects without an AMI) and, especially in case of the $0 \mathrm{~h} / 1 \mathrm{~h}$ algorithm, assay-dependent. Second, we showed that the difference in diagnostic accuracy between the combined and single cTn measurements is numerically small (except for ratio). In addition, the results of the reclassification statistics indicated that the application of two cTn isoforms at presentation may add incremental value, but that this is not the case for the mathematical derivations when applied to the whole cohort. Third, combining cardiac hscTnl and hs-cTnT, using the mathematical derivations sum and product or a combination algorithm, may overcome limitations of the individual assays and lead to a 3-to-6-fold increase in the number of rule-outs after a single blood draw (Figure 3). 


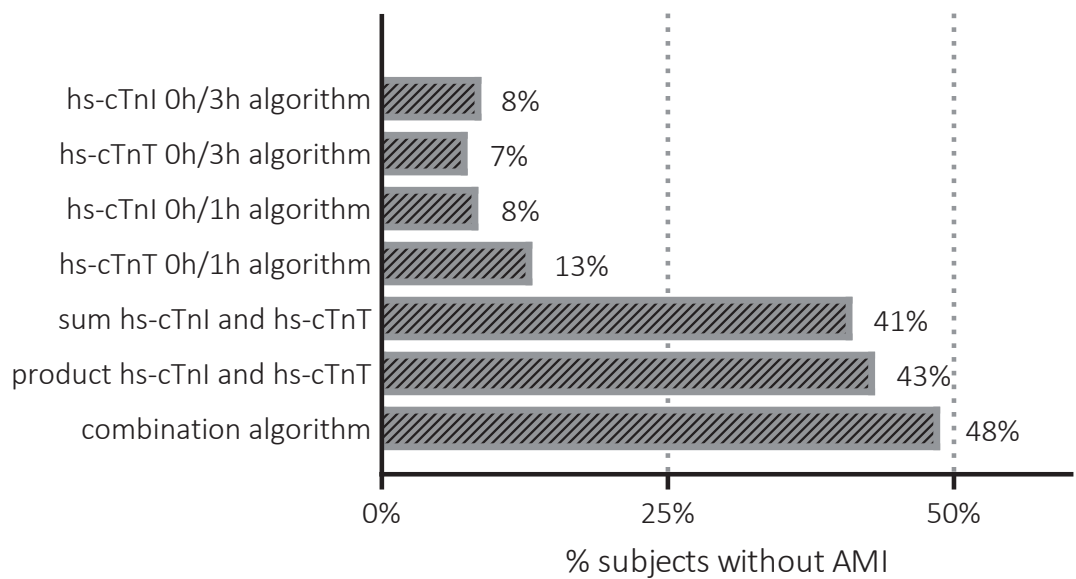

Figure 3. Percentage of patients without AMI in which AMI can be ruled-out after a single blood draw at presentation in the APACE cohort

The findings from this study corroborate and extend previous work aiming to further improve the safety and efficacy of the rule-out and rule-in of AMI among patients presenting with acute chest discomfort to the $\mathrm{ED}^{2-10,23,37}$. To the best of our knowledge this work is the first systematic approach testing the clinical utility of mathematical combinations of hs-cTnl and hs-cTnT, the two most accurate biochemical signals in the early diagnosis of $\mathrm{AMI}^{1-10}$. While there is broad agreement that hs-cTnl or hs-cTnT should be used as a key component in any AMI rule-out algorithm 2,9,12,38,39, it has remained unclear whether a second biochemical signature could provide enough incremental value to potentially justify routine clinical use. The novel concept investigated in this study was based on recent studies documenting at times remarkable differences between the cTnl and the cTnT signal, and the moderate agreement between clinical decisions made on these concentrations ${ }^{16,40,41}$. We hypothesized that combining the two biochemical signals might overcome independent pathophysiological, pre-analytical and analytical differences between the individual molecules such as (auto)antibodies and suggested interference with troponin released from skeletal muscle $15,42-46$, and might therefore have higher diagnostic accuracy for AMI than either cTnl or cTnT alone. 
This study shows that combining hs-cTnl and hs-cTnT may contribute to a significant increase in the number of rule-outs at presentation. The small increase in false-negative results when the derived thresholds were applied in the external validation cohort raises the question what is considered a still acceptable number of false rule-ins and rule-outs ${ }^{47}$. Furthermore, it illustrates the outlier-dependency of the determination of very low cut-off values, and advocates the use of extended (pooled) cohorts and the recalibration of cut-off values for the determination of more universally applicable decision rules ${ }^{48}$. A second point that merits attention are the, at first sight contrary, unconvincing results of the diagnostic accuracy and reclassification statistics. These findings are of limited additional value for the whole population, whereas combing hs-cTnl and hs-cTnT might be especially valuable in patients with low hs-cTn concentrations at presentation. Another reason for this discrepancy might be the three-group (rule-out, observational, rule-in) approach that is used for the diagnosis of $\mathrm{AMI}$ and its outlier dependency.

The clinical implementation of a dual-marker approach combining cTnl and cTnT would likely be associated with substantial logistic obstacles since no diagnostic company currently is able to provide both hs-cTnT and hs-cTnl assays on the same laboratory platform. In addition, most hospitals currently do not have analyzers for both analytes running on a 24/7 basis. Therefore, the cost-effective clinical implementation of the dual-marker approach would require a collaboration among diagnostic companies for the provision of both hs-cTnT and hs-cTnl assays on the analyzer that is used for clinical chemistry routine. Nevertheless, rapid and safe clinical decision making based on a single hs-cTn measurement at presentation seems to be approaching its limits, and the exploration of new diagnostic strategies including combinations of biomarkers or imaging seems to be indicated ${ }^{10}$. From this point of view, overcoming these logistic obstacles by close collaboration between diagnostic companies, hospital laboratories, medical doctors and researchers would be able to provide substantial medical value for patients and physicians, and economic value for hospitals and the health care system in general. Future studies are necessary to identify the best strategy and to better quantify the possible clinical benefit associated with the combination of cTnl and cTnT. Considering the relevant unmet clinical need as quantified by the high percentage of rule- 
out mismatches, the substantial increase in early rule-outs compared to the current ESC Oh/1h-algorithm and the substantial cost savings associated with reductions in the length of stay in the $\mathrm{ED}^{49}$, dedicated economic analyses can be expected to show substantial reductions in time to decision, time to discharge, and therefore treatment costs. Furthermore, it is important to highlight that despite the very high diagnostic accuracy, hscTn and their combinations will always have to be used clinically only in conjunction with full clinical assessment including detailed patient history, physical examination, and the $\mathrm{ECG}^{2}$. Some limitations of this study merit consideration. First, the central adjudication by two independent cardiologists based on the clinical dataset including cardiac imaging and serial measurements of the local (hs)-cTn and the study-specific dataset including 34 chest pain characteristics, serial measurements of hs-cTnT and follow-up in the APACE study represents the highest quality possible in a diagnostic study. However, it possibly introduced a very small but unavoidable disadvantage for hs-cTnl regarding diagnostic accuracy. This is at large counterbalanced by the use of s-cTnl for the adjudication in ADAPT, as this possibly introduced a very small but unavoidable disadvantage for hs-cTnT regarding diagnostic accuracy. Second, we cannot exactly quantify the cost-effectiveness of this novel approach. Third, patients with terminal kidney failure on chronic dialysis were excluded from APACE. Accordingly, we cannot comment on the possible clinical utility of the combination approach in these vulnerable patients.

In conclusion, diagnostic strategies combining cTnl and cTnT measurements, mathematical derivations and a combination algorithm, may significantly increase the number of patients eligible for very early and safe rule-out, but does not seem helpful for the rule-in of AMI. 


\section{References}

1. Nawar EW, Niska RW, Xu J. National Hospital Ambulatory Medical Care Survey: 2005 emergency department summary. Adv Data 2007:1-32.

2. Roffi M, Patrono C, Collet JP, et al. 2015 ESC Guidelines for the management of acute coronary syndromes in patients presenting without persistent ST-segment elevation: Task Force for the Management of Acute Coronary Syndromes in Patients Presenting without Persistent ST-Segment Elevation of the European Society of Cardiology (ESC). Eur Heart J 2016;37:267-315.

3. Reichlin T, Hochholzer W, Bassetti S, et al. Early diagnosis of myocardial infarction with sensitive cardiac troponin assays. N Engl J Med 2009;361:858-67.

4. Reiter M, Twerenbold R, Reichlin T, et al. Early diagnosis of acute myocardial infarction in the elderly using more sensitive cardiac troponin assays. Eur Heart J 2011;32:1379-89.

5. Raskovalova T, Twerenbold R, Collinson PO, et al. Diagnostic accuracy of combined cardiac troponin and copeptin assessment for early rule-out of myocardial infarction: a systematic review and meta-analysis. Eur Heart J Acute Cardiovasc Care 2014;3:18-27.

6. Lipinski MJ, Baker NC, Escarcega RO, et al. Comparison of conventional and high-sensitivity troponin in patients with chest pain: a collaborative meta-analysis. Am Heart J 2015;169:6-16 e6.

7. Mockel M, Searle J, Hamm C, et al. Early discharge using single cardiac troponin and copeptin testing in patients with suspected acute coronary syndrome (ACS): a randomized, controlled clinical process study. Eur Heart J 2015;36:369-76.

8. Collinson P, Gaze D, Goodacre S. Comparison of contemporary troponin assays with the novel biomarkers, heart fatty acid binding protein and copeptin, for the early confirmation or exclusion of myocardial infarction in patients presenting to the emergency department with chest pain. Heart 2014;100:140-5.

9. Thygesen K, Mair J, Giannitsis E, et al. How to use high-sensitivity cardiac troponins in acute cardiac care. Eur Heart J 2012;33:2252-7.

10. Nestelberger T, Wildi K, Boeddinghaus J, et al. Characterization of the observe zone of the ESC 2015 highsensitivity cardiac troponin 0h/1h-algorithm for the early diagnosis of acute myocardial infarction. Int J Cardiol 2016;207:238-45.

11. Amsterdam EA, Wenger NK, Brindis RG, et al. 2014 AHA/ACC Guideline for the Management of Patients with Non-ST-Elevation Acute Coronary Syndromes: a report of the American College of Cardiology/American Heart Association Task Force on Practice Guidelines. J Am Coll Cardiol 2014;64:e139-228.

12. Thygesen K, Mair J, Katus $\mathrm{H}$, et al. Recommendations for the use of cardiac troponin measurement in acute cardiac care. Eur Heart J 2010;31:2197-204.

13. Katrukha IA. Human cardiac troponin complex. Structure and functions. Biochemistry (Mosc) 2013;78:1447-65.

14. Wu AH, Feng YJ. Biochemical differences between cTnT and cTnl and their significance for diagnosis of acute coronary syndromes. Eur Heart J 1998;19 Suppl N:N25-9.

15. Rubini Gimenez M, Twerenbold R, Reichlin T, et al. Direct comparison of high-sensitivity-cardiac troponin I vs. T for the early diagnosis of acute myocardial infarction. European heart journal 2014;35:2303-11.

16. Kimenai DM, Henry RM, van der Kallen CJ, et al. Direct comparison of clinical decision limits for cardiac troponin T and I. Heart 2016;102:610-6.

17. Wildi K, Gimenez MR, Twerenbold R, et al. Misdiagnosis of Myocardial Infarction Related to Limitations of the Current Regulatory Approach to Define Clinical Decision Values for Cardiac Troponin. Circulation 2015;131:2032-40. 18. Inker LA, Schmid CH, Tighiouart $\mathrm{H}$, et al. Estimating glomerular filtration rate from serum creatinine and cystatin C. N Engl J Med 2012;367:20-9.

19. Haaf $P$, Reichlin $T$, Twerenbold $R$, et al. Risk stratification in patients with acute chest pain using three highsensitivity cardiac troponin assays. Eur Heart J 2014;35:365-75.

20. Reiter M, Twerenbold R, Reichlin $T$, et al. Early diagnosis of acute myocardial infarction in patients with preexisting coronary artery disease using more sensitive cardiac troponin assays. Eur Heart J 2012;33:988-97. 
21. Than M, Cullen L, Aldous S, et al. 2-Hour accelerated diagnostic protocol to assess patients with chest pain symptoms using contemporary troponins as the only biomarker: the ADAPT trial. J Am Coll Cardiol 2012;59:2091-8. 22. Luepker RV, Apple FS, Christenson RH, et al. Case definitions for acute coronary heart disease in epidemiology and clinical research studies: a statement from the AHA Council on Epidemiology and Prevention; AHA Statistics Committee; World Heart Federation Council on Epidemiology and Prevention; the European Society of Cardiology Working Group on Epidemiology and Prevention; Centers for Disease Control and Prevention; and the National Heart, Lung, and Blood Institute. Circulation 2003;108:2543-9.

23. Thygesen K, Alpert JS, White HD, Joint ESCAAHAWHFTFftRoMI. Universal definition of myocardial infarction. Eur Heart J 2007;28:2525-38.

24. Apple FS, Wu AH, Jaffe AS. European Society of Cardiology and American College of Cardiology guidelines for redefinition of myocardial infarction: how to use existing assays clinically and for clinical trials. Am Heart J 2002;144:981-6.

25. Apple FS, Wu AH, Jaffe AS, et al. National Academy of Clinical Biochemistry and IFCC Committee for Standardization of Markers of Cardiac Damage Laboratory Medicine practice guidelines: Analytical issues for biomarkers of heart failure. Circulation 2007;116:e95-8.

26. Koerbin G, Tate J, Potter JM, Cavanaugh J, Glasgow N, Hickman PE. Characterisation of a highly sensitive troponin I assay and its application to a cardio-healthy population. Clin Chem Lab Med 2012;50:871-8.

27. Giannitsis E, Kurz K, Hallermayer K, Jarausch J, Jaffe AS, Katus HA. Analytical validation of a high-sensitivity cardiac troponin T assay. Clin Chem 2010;56:254-61.

28. Wildi K, Twerenbold R, Jaeger C, et al. Clinical impact of the 2010-2012 low-end shift of high-sensitivity cardiac troponin T. Eur Heart J Acute Cardiovasc Care 2016.

29. Hallermayer K, Jarausch J, Menassanch-Volker S, Zaugg C, Ziegler A. Implications of adjustment of high-sensitivity cardiac troponin T assay. Clin Chem 2013;59:572-4.

30. Kavsak PA, Hill SA, McQueen MJ, Devereaux PJ. Implications of adjustment of high-sensitivity cardiac troponin T assay. Clin Chem 2013;59:574-6.

31. Levey AS, Coresh J, Greene T, et al. Using standardized serum creatinine values in the modification of diet in renal disease study equation for estimating glomerular filtration rate. Ann Intern Med 2006;145:247-54.

32. Hanley JA, McNeil BJ. The meaning and use of the area under a receiver operating characteristic (ROC) curve. Radiology 1982;143:29-36.

33. Hanley JA, McNeil BJ. A method of comparing the areas under receiver operating characteristic curves derived from the same cases. Radiology 1983;148:839-43.

34. Reichlin T, Schindler C, Drexler B, et al. One-hour rule-out and rule-in of acute myocardial infarction using highsensitivity cardiac troponin T. Arch Intern Med 2012;172:1211-8.

35. Jaeger $C$, Wildi $K$, Twerenbold R, et al. One-hour rule-in and rule-out of acute myocardial infarction using highsensitivity cardiac troponin I. Am Heart J 2016;171:92-102 e1-5.

36. Zellweger C, Wildi K, Twerenbold R, et al. Use of copeptin and high-sensitive cardiac troponin T for diagnosis and prognosis in patients with diabetes mellitus and suspected acute myocardial infarction. Int J Cardiol 2015;190:1907.

37. Stallone F, Schoenenberger AW, Puelacher C, et al. Incremental value of copeptin in suspected acute myocardial infarction very early after symptom onset. Eur Heart J Acute Cardiovasc Care 2016.

38. Hollander JE, Than M, Mueller C. State-of-the-Art Evaluation of Emergency Department Patients Presenting With Potential Acute Coronary Syndromes. Circulation 2016;134:547-64.

39. Mueller C, Giannitsis E, Mockel M, et al. Rapid rule out of acute myocardial infarction: novel biomarker-based strategies. Eur Heart J Acute Cardiovasc Care 2016.

40. Reichlin T, Cullen L, Parsonage WA, et al. Two-hour algorithm for triage toward rule-out and rule-in of acute myocardial infarction using high-sensitivity cardiac troponin T. Am J Med 2015;128:369-79 e4.

41. Mueller C, Giannitsis E, Christ M, et al. Multicenter Evaluation of a 0-Hour/1-Hour Algorithm in the Diagnosis of Myocardial Infarction With High-Sensitivity Cardiac Troponin T. Ann Emerg Med 2016;68:76-87 e4. 
42. Guclu T, Bolat S, Senes M, Yucel D. Relationship between high sensitivity troponins and estimated glomerular filtration rate. Clin Biochem 2016;49:467-71.

43. Savukoski T, Ilva T, Lund J, et al. Autoantibody prevalence with an improved immunoassay for detecting cardiac troponin-specific autoantibodies. Clin Chem Lab Med 2014;52:273-9.

44. Adamczyk M, Brashear RJ, Mattingly PG. Circulating cardiac troponin-I autoantibodies in human plasma and serum. Ann N Y Acad Sci 2009;1173:67-74.

45. Adamczyk M, Brashear RJ, Mattingly PG. Prevalence of autoantibodies to cardiac troponin T in healthy blood donors. Clin Chem 2009;55:1592-3.

46. Lippi G, Aloe R, Meschi T, Borghi L, Cervellin G. Interference from heterophilic antibodies in troponin testing. Case report and systematic review of the literature. Clin Chim Acta 2013;426:79-84.

47. Than M, Herbert M, Flaws D, et al. What is an acceptable risk of major adverse cardiac event in chest pain patients soon after discharge from the Emergency Department?: a clinical survey. Int J Cardiol 2013;166:752-4.

48. Pickering JW, Than MP. The small number problem in diagnostic algorithms and why we need to bootstrap. Clin Biochem 2017.

49. Shortt C, Xie F, Whitlock R, et al. Economic Considerations of Early Rule-In/Rule-Out Algorithms for The Diagnosis of Myocardial Infarction in The Emergency Department Using Cardiac Troponin and Glycemic Biomarkers. Clin Chem 2017;63:593-60. 


\section{Supplemental information}

\section{Supplemental Methods}

\section{Adjudication of final diagnoses in APACE cohort}

The cTn assays used clinically in most of the participating institutions changed during the study from a conventional cTn assay to the hs-cTnT assay. In order to take advantage of the higher sensitivity and higher overall diagnostic accuracy offered by the hs-cTnT assay, patients were adjudicated using the hs-cTnT values in all patients. In patients in whom clinically a conventional cTn assay was used, the conventional cTn values and the hs-cTnT values were available for the adjudication. In patients in whom clinically the hs-cTnT assay was used, only the hs-cTnT values were available for the adjudication.

The following conventional cTn assays were used: For the Roche cTnT 4th generation assay, the 10\% $\mathrm{CV}$ level is $0.035 \mathrm{ug} / \mathrm{l}$. The laboratories of the participating sites reported only two decimals; therefore $0.04 \mathrm{ug} / \mathrm{I}$ was used as a cut-off for myocardial necrosis. In order to fulfil the criteria of a significant change ( $30 \%$ of 99 th percentile or $10 \% \mathrm{CV}$ level), a patient would e.g. need to have a level of $<0.01 \mathrm{ug} / \mathrm{l}$ at presentation and $0.04 \mathrm{ug} / \mathrm{l}$ at $6 \mathrm{~h}$. A patient would also qualify if the first level is $0.02 \mathrm{ug} / \mathrm{l}$ and the second $0.04 \mathrm{ug} / \mathrm{l}$. A patient would not fulfil the criteria if the first level is $0.03 \mathrm{ug} / \mathrm{l}$ and the second is $0.04 \mathrm{ug} / \mathrm{I}$. If the first level is $0.04 \mathrm{ug} / \mathrm{l}$, the second level needs to be at least $0.06 \mathrm{ug} / \mathrm{l}$.

For the Abbott Axsym cTnI ADV, the $10 \%$ CV level is $0.16 \mathrm{ug} / \mathrm{l}$. A patient having $0.16 \mathrm{ug} / \mathrm{l}$ at presentation would meet the criteria for significant change if the second was $\geq 0.21 \mathrm{ug} / \mathrm{l}$. A patient having $<0.12 \mathrm{ug} / \mathrm{l}$ at presentation (limit of detection) would qualify if the second is $>0.16 \mathrm{ug} / \mathrm{l}$.

For the Beckmann Coulter Accu CTnl, the $10 \%$ CV level is $0.06 \mathrm{ug} / \mathrm{I}$. A patient having $0.06 \mathrm{ug} / \mathrm{l}$ at presentation would qualify if the second is $\geq 0.08 \mathrm{ug} / \mathrm{l}$. A patient having 0.05 at presentation would qualify if the second is $0.07 \mathrm{ug} / \mathrm{l}$, but not $0.06 \mathrm{ug} / \mathrm{l}$. A patient having undetectable $\mathrm{cTnl}(\mathrm{cTnl}<0.01 \mathrm{ug} / \mathrm{l}$ ) at presentation would qualify if the second is $\geq 0.06 \mathrm{ug} / \mathrm{l}$.

For hs-cTnT the 99th percentile (14ng/l) was used as cut-off for myocardial necrosis ${ }^{1,2}$. Absolute changes in hs-cTnT were used to determine significant changes based on the diagnostic superiority of absolute over relative changes ${ }^{3,4}$. Based on studies of the biological variation of $\mathrm{cTn}^{5,6}$ as well as on data from previous chest pain cohort studies 7,8 , a significant absolute change was defined as a rise or fall of at least 10ng/I within six hours. In patients, in whom a 6 hour hs-cTnT level was not available, changes were assessed at earlier time points. In an assumption of linearity, an absolute change of $6 \mathrm{ng} / \mathrm{I}$ within three hours was considered. 


\section{Adjudication of final diagnoses in ADAPT cohort}

Outcomes were adjudicated independently by local cardiologists using predefined standardized reporting guidelines. Cardiologists had knowledge of the clinical record, ECG, troponin results and objective testing from standard care. A second cardiologist conducted a blind review of all ACS cases and $10 \%$ of non-ACS cases. In cases of disagreement, endpoints were agreed by consensus.

Diagnosis of AMI was according to international guidelines and based on evidence of myocardial necrosis and ischemia. Evidence of ischaemia included at least one of ECG changes or positive imaging results from exercise tolerance testing, myocardial perfusion scan, stress echocardiography, computed tomographic coronary angiography or coronary angiography during catheterization. Necrosis was diagnosed based on a rise or fall of cardiac troponin concentration over at least six hours with at least one value above the 99th percentile of the normal reference range at a level of assay imprecision near to $10 \%$. If the troponin was greater than the reference range but no rise or fall was recorded, other causes of raised troponin were considered. If no alternative cause for the troponin rise was apparent and if the clinical presentation was suggestive of ACS, an adjudicated diagnosis of AMI was made.

Emergency revascularization was defined as $\mathrm{PCl}$ or $\mathrm{CABG}$ in a symptomatic patient where the clinical status includes either 1) ischaemic dysfunction (ongoing ischaemia despite maximal medical therapy, acute evolving myocardial within 24 hours before intervention or pulmonary oedema requiring intubation) or 2) mechanical dysfunction (shock with or without circulatory support). Urgent revascularisation included $\mathrm{PCI}$ or $\mathrm{CABG}$ that did not meet the emergency criteria above but was required during the same hospitalization to minimize chance of further clinical deterioration. Elective revascularization, or those procedures that could be deferred without increased risk of compromised cardiovascular outcome, were not included in the endpoint.

\section{Assumption of linearity of absolute changes of hs-cTnT within the first hours}

The assumption of linearity of absolute changes within the first hours is based on unpublished internal data as well as recent data from Ola Hammarsten et al. showing a near-linear increase in levels of hscTnT with increasing time from symptom onset in their NSTEMI cohort 9.

\section{Determination of cut-offs for the combination algorithm}

We aimed to determine the safe and optimal combination of hs-cTnl concentration and hs-cTnT concentration. A test was considered positive if either hs-cTn concentration was greater than or equal to an hs-cTn threshold for that troponin assay. We varied the hs-cTn threshold for each troponin assay in steps of $0.1 \mathrm{ng} / \mathrm{L}$ across the range from the LoD to $26 \mathrm{ng} / \mathrm{L}$. At each combination of hs-cTn thresholds 
we calculated the sensitivity and specificity for the diagnosis of AMI. We created 500 bootstraps of the data, and to average the results we then fitted a smooth surface (thin plate spline) for sensitivity on the hs-cTnl threshold - hs-cTnT threshold grid from which we could determine the $99 \%$ sensitivity contour and contours for specificity. The optimal threshold was defined as the combination with $99 \%$ sensitivity and maximized specificity. 


\section{Supplemental Figures}

A

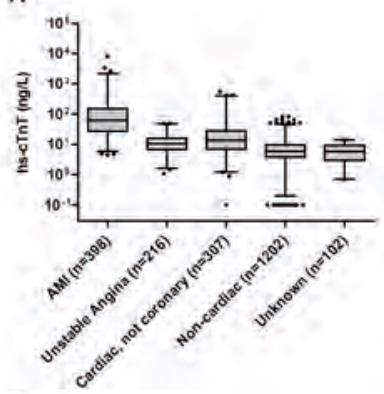

C

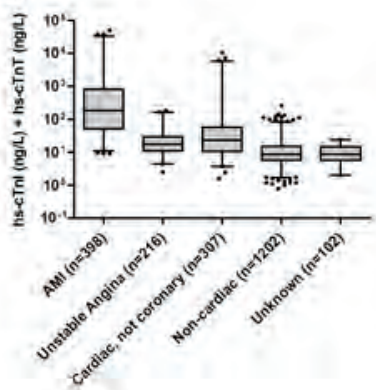

B

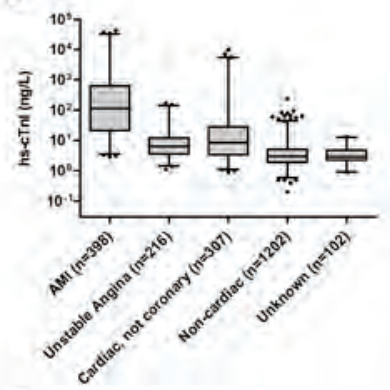

D

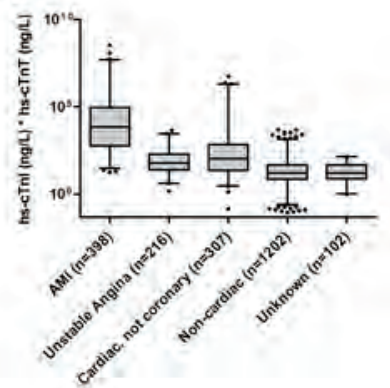

E

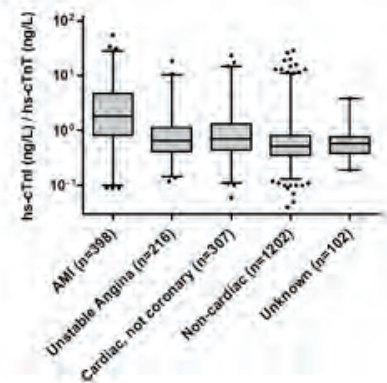

Supplementary Figure 1. hs-cTn concentrations at presentation in the APACE cohort. The boxes represent median and inter-quartile ranges, the whiskers 1st-99th percentile of A) cardiac troponin T (hs-cTnT), B) cardiac troponin I (hs-cTnl), C) sum, D) product and E) ratio. hs-cTn denotes high-sensitivity cardiac troponin; hs-cTnl,high-sensitivity cardiac troponin I; AMI, acute myocardial infarction; UA, unstable angina; $\mathrm{CNC}$, cardiac non-coronary disease; NCCP, non-cardiac chest pain. 


\section{Supplemental Tables}

Supplementary Table 1. Baseline characteristics of patients in the APACE cohort

\begin{tabular}{|c|c|c|c|}
\hline & All $(n=2225)$ & AMI (n=398) & No AMI $(n=1827)$ \\
\hline Age, y & $62(49-75)$ & $72(61-80)$ & $60(47-73)$ \\
\hline Male sex, n (\%) & $1511(68)$ & $290(73)$ & $1221(67)$ \\
\hline eGFR, $\mathrm{ml} \cdot \mathrm{min}^{-1} \cdot 1.73 \mathrm{~m}^{-2}$ & $85(69-101)$ & $73(55-93)$ & $87(71-103)$ \\
\hline Body-mass index, $\mathrm{kg} / \mathrm{m}^{2}$ & $26(24-30)$ & $26(24-29)$ & $26(24-30)$ \\
\hline \multicolumn{4}{|l|}{ Risk factors, n (\%) } \\
\hline Hypertension & $1382(62)$ & $318(80)$ & $1064(58)$ \\
\hline Hypercholesterolemia & $1110(50)$ & $272(68)$ & $838(46)$ \\
\hline Diabetes Mellitus & $384(17)$ & $108(27)$ & $276(15)$ \\
\hline Current or previous smoking & $1366(61)$ & $253(64)$ & $1113(61)$ \\
\hline Family history & $549(25)$ & $117(29)$ & $432(24)$ \\
\hline \multicolumn{4}{|l|}{ History, n (\%) } \\
\hline Coronary Artery Disease & $776(35)$ & $202(51)$ & $574(31)$ \\
\hline Previous AMI & $513(23)$ & $136(34)$ & $377(21)$ \\
\hline Previous revascularisation & $612(28)$ & $147(37)$ & $465(26)$ \\
\hline Peripheral artery disease & $141(6)$ & $56(14)$ & $85(5)$ \\
\hline Previous stroke & $122(6)$ & $39(10)$ & $83(5)$ \\
\hline \multicolumn{4}{|l|}{ ECG changes, $n(\%)$} \\
\hline ST segment depression & $232(10)$ & $121(30)$ & $111(6)$ \\
\hline T wave inversion & $296(13)$ & $100(25)$ & $196(11)$ \\
\hline LBBB & $25(1)$ & $11(3)$ & $14(1)$ \\
\hline \multicolumn{4}{|l|}{ Medication at presentation, $\mathrm{n}(\%)$} \\
\hline ASA & $808(36)$ & $199(50)$ & $609(33)$ \\
\hline Vitamin $\mathrm{K}$ antagonist & $191(9)$ & $41(10)$ & $150(8)$ \\
\hline$\beta$-blockers & $770(35)$ & $174(44)$ & $596(33)$ \\
\hline Statins & $777(35)$ & $178(45)$ & $599(33)$ \\
\hline ACEIs/ARBs & $842(38)$ & $206(52)$ & $636(35)$ \\
\hline Calcium antagonists & $321(14)$ & $82(21)$ & $239(13)$ \\
\hline Nitrates & $257(12)$ & $85(21)$ & $172(9)$ \\
\hline
\end{tabular}

APACE denotes Advantageous Predictors of Acute Coronary Syndrome Evaluation; AMI, acute myocardial infarction; ECG, electrocardiogram; eGFR, estimated glomerular filtration rate; LBBB, left bundle branch block; ASA, acetyl salicylic acid; ACEI, angiotensin-converting enzyme inhibitor; ARB, angiotensin receptor blocker. Values are expressed in percentage or medians \pm IQR. 
Supplementary Table 2. Baseline characteristics of patients in the ADAPT cohort

\begin{tabular}{llll}
\hline & All $(\mathrm{n}=2537)$ & AMI $(\mathrm{n}=408)$ & No AMI $(\mathrm{n}=2129)$ \\
\hline Age, $y$ & $60(51-71)$ & $59(49-69)$ & $69(59-78)$ \\
Male sex, $\mathrm{n}(\%)$ & $1535(61)$ & $292(72)$ & $1243(58)$ \\
Body-mass index, $\mathrm{kg} / \mathrm{m}^{2}$ & $27(24-31)$ & $28(24-31)$ & $27(24-31)$ \\
Risk factors, $\mathrm{n}(\%)$ & & & $1062(50)$ \\
Hypertension & $1316(52)$ & $254(62)$ & $1081(51)$ \\
Hypercholesterolemia & $1309(52)$ & $228(56)$ & $296(14)$ \\
Diabetes Mellitus & $377(15)$ & $81(20)$ & $390(18)$ \\
Current smoking & $462(18)$ & $72(18)$ & $1148(54)$ \\
Family history of CAD & $1393(55)$ & $245(60)$ & \\
History, $\mathrm{n}$ (\%) & & & $499(23)$ \\
Previous AMI & $627(25)$ & $128(31)$ & $440(21)$ \\
Previous PCl & $525(21)$ & $85(21)$ & $164(8)$ \\
Previous CABG & $219(9)$ & $55(13)$ & $69(3)$ \\
Peripheral artery disease & $94(4)$ & $25(6)$ & $166(8)$ \\
Previous stroke & $205(8)$ & $39(10)$ &
\end{tabular}

ADAPT denotes Accelerated Diagnostic Protocol to Assess Patients Witch Chest Pain Symptoms Using Contemporary Troponins as the Only Biomarker; AMI, acute myocardial infarction; CABG, coronary artery bypass grafting; PCI, percutaneous coronary intervention. Values are expressed in percentage or medians $\pm I Q R$. 
Supplementary Table 3. hs-cTn concentrations at presentation in the APACE cohort

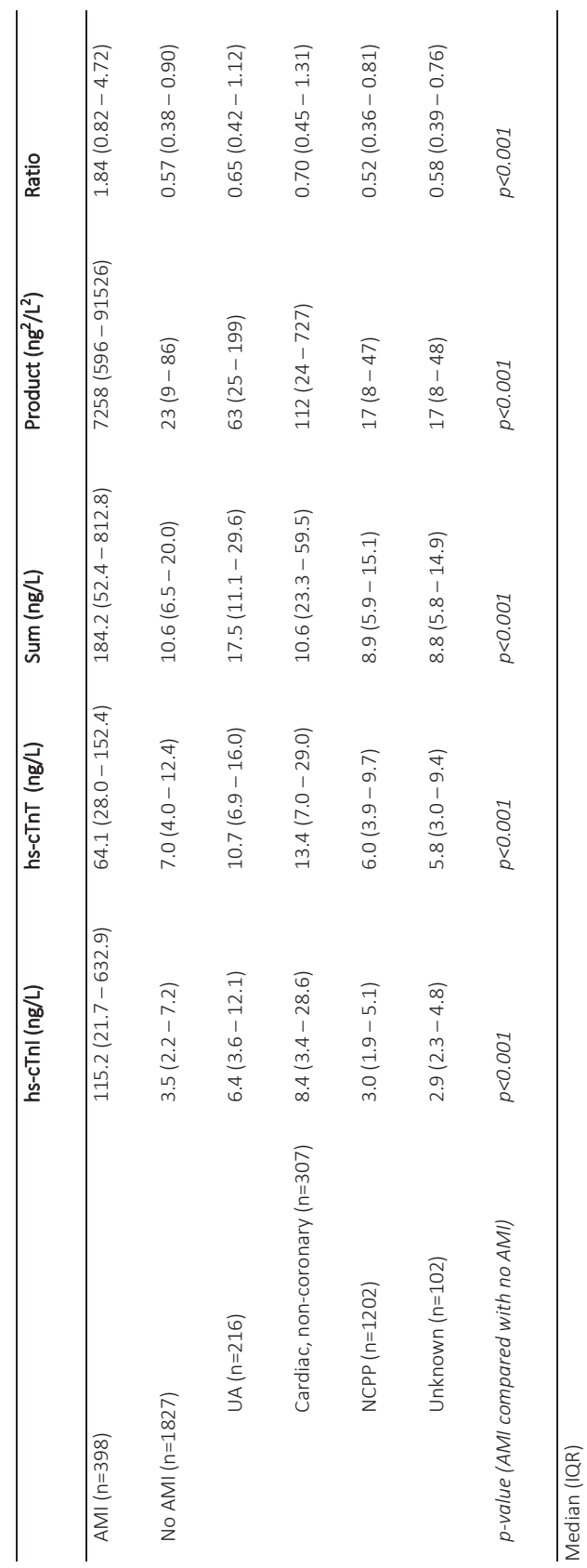


Supplementary Table 4a: Agreement on rule-out at presentation using the Oh/3h-algorithm in the APACE cohort

\begin{tabular}{l|lcc|}
\multicolumn{3}{c}{} & \multicolumn{2}{c|}{ hs-cTnT } \\
\cline { 2 - 4 } \multicolumn{1}{c|}{ hs-cTnl } & rule-out* & no rule-out \\
& rule-out* & $132(5.9 \%)$ & $25(1.1 \%)$ \\
no rule-out & $1(0.0 \%)$ & $2067(92.9 \%)$ \\
\hline
\end{tabular}

* if hs-cTnT $<14 \mathrm{ng} / \mathrm{L}$ or $h s-c T n l<26.2 \mathrm{ng} / \mathrm{L},>6 \mathrm{~h}$ after onset of symptoms and painfree, and GRACE score $<140$

Supplementary Table 4b: Agreement on rule-out at presentation using the $0 \mathrm{~h} / 1 \mathrm{~h}$-algorithm in the APACE cohort

\begin{tabular}{l|lcc|}
\multicolumn{3}{c}{} & \multicolumn{2}{c|}{ hs-cTnT } \\
\cline { 2 - 4 } \multicolumn{1}{c|}{ hs-cTnl } & rule-out* & no rule-out \\
& rule-out* & $98(4.4 \%)$ & $51(2.3 \%)$ \\
no rule-out & $137(6.2 \%)$ & $1939(87.1 \%)$ \\
\hline
\end{tabular}

* if hs-cTnT $<5 \mathrm{ng} / \mathrm{L}$ or $\mathrm{hs}-\mathrm{cTn} /<2 \mathrm{ng} / \mathrm{L}$ and $>3 \mathrm{~h}$ after onset of symptoms

Supplementary Table 4c: Agreement on rule-in at presentation using the 0/1h-algorithm in the APACE cohort

\begin{tabular}{c|lll|}
\multicolumn{1}{c}{} & \multicolumn{2}{c}{ hs-cTnT } \\
\cline { 2 - 4 } & & \multicolumn{1}{c}{ rule-in* } & no rule-in \\
hs-cTnl & rule-in* & $246(11.1 \%)$ & $85(3.8 \%)$ \\
& no rule-in & $27(1.2 \%)$ & $1867(83.9 \%)$ \\
\hline
\end{tabular}

* if hs-cTnT > $52 \mathrm{ng} / \mathrm{L}$ or hs-cTnl $>52 \mathrm{ng} / \mathrm{L}$ 
Supplementary Table 5. Diagnostic accuracy of hs-cTnT and hs-cTnl after the addition of the alternative signal or sum, product or ratio at presentation in the APACE cohort

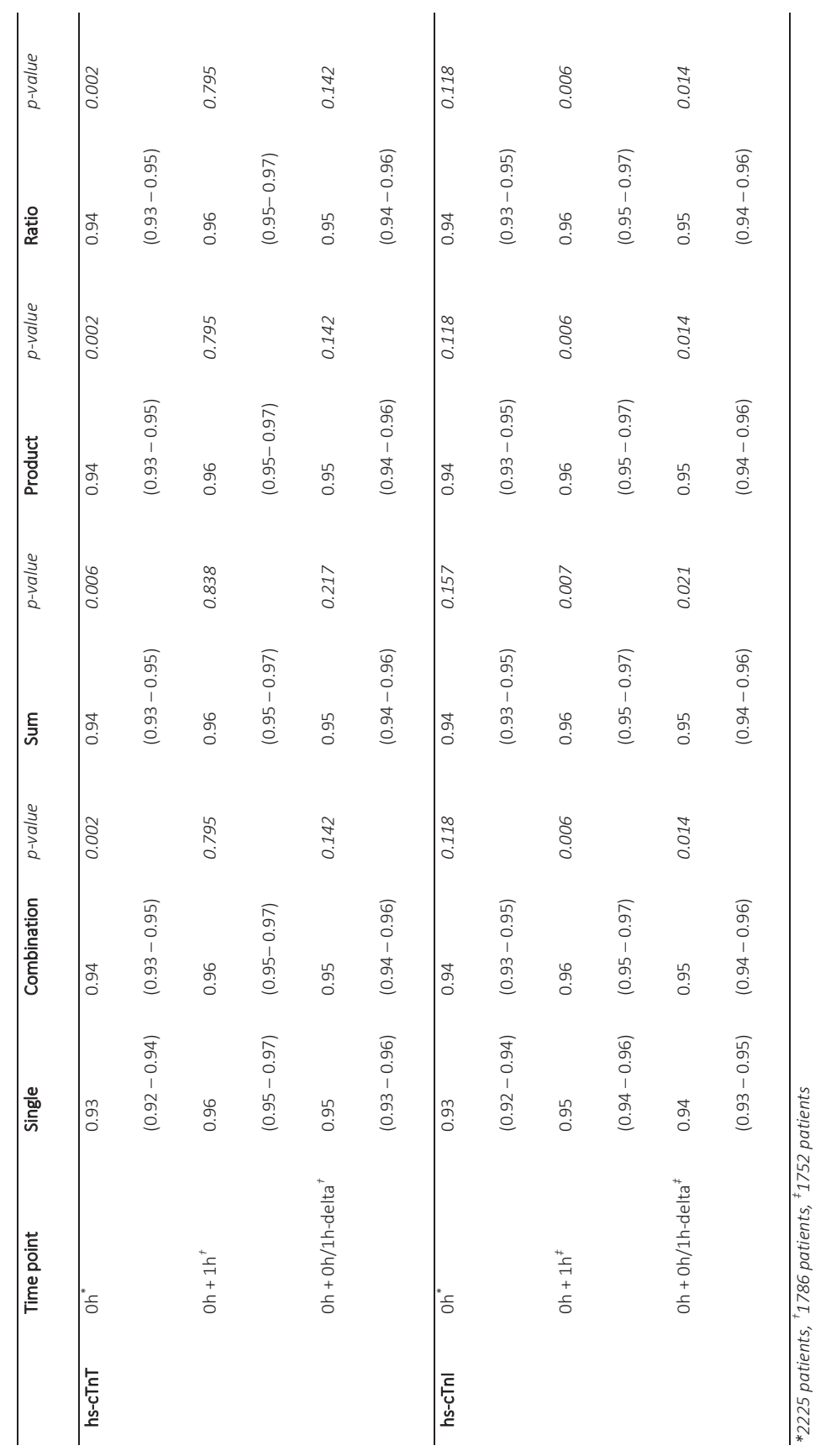


Cardiac Troponins: State of the (He)Art

Supplementary Table 6a. Absolute IDI at presentation in the APACE cohort

\begin{tabular}{|c|c|c|c|c|c|c|c|c|}
\hline & Combination & p-value & Sum & $p$-value & Product & $p$-value & Ratio & p-value \\
\hline \multirow[t]{2}{*}{ hs-cTnl } & 0.214 & $<0.001$ & 0.038 & $<0.001$ & -0.100 & 1.00 & -0.083 & 1.00 \\
\hline & $(0.183 ; 0.244)$ & & $(0.011 ; 0.065)$ & & $(-0.128 ;-0.073)$ & & $(-0.113 ;-0.052)$ & \\
\hline \multirow[t]{2}{*}{ hs-cTnT } & 0.016 & 0.23 & -0.159 & 1.00 & -0.298 & 1.00 & -0.280 & 1.00 \\
\hline & $(-0.011 ; 0.043)$ & & $(-0.188 ;-0.130)$ & & $(-0.328 ;-0.267)$ & & $(-0.311 ;-0.248)$ & \\
\hline
\end{tabular}

dedicates hs-cTnT resp. hs-cTnl alone (old model) versus the mathematical combinations of hs-cTnT and hs-cTnl (new model)

Supplementary Table 6b. Category-free NRI at presentation in the APACE cohort

\begin{tabular}{cccccccc}
\hline & Combination & p-value & Sum & p-value & Product & p-value & Ratio \\
\hline hs-cTnl & $121.1 \%$ & $<0.001$ & $127.5 \%$ & $<0.001$ & $-100.6 \%$ & 1.00 & $-15.8 \%$ \\
hs-cTnT & $105.1 \%$ & $<0.001$ & $-123.0 \%$ & 1.00 & $-131.7 \%$ & 1.00 & $-133.0 \%$ \\
\hline
\end{tabular}

dedicates hs-cTnT resp. hs-cTnl alone (old model) versus the mathematical combinations of hs-cTnT and hs-cTnl (new model) 


\section{Supplemental references}

1. Apple FS, Wu AH, Jaffe AS et al. European Society of Cardiology and American College of Cardiology guidelines for redefinition of myocardial infarction: how to use existing assays clinically and for clinical trials. Am Heart J. 2002;144:981-986.

2. Apple FS, Jesse RL, Newby LK, Wu AHB, Christenson RH. National Academy of Clinical Biochemistry and IFCC Committee for Standardization of Markers of Cardiac Damage Laboratory Medicine Practice Guidelines: Analytical issues for biochemical markers of acute coronary syndromes. Circulation. 2007;115:e352-5.

3. Reichlin T, Irfan A, Twerenbold R et al. Utility of absolute and relative changes in cardiac troponin concentrations in the early diagnosis of acute myocardial infarction. Circulation. 2011;124:136-45.

4. Thygesen K, Mair J, Giannitsis E et al. The Study Group on Biomarkers in Cardiology of the ESCWG on ACC. How to use high-sensitivity cardiac troponins in acute cardiac care. Eur Heart J. 2012;33:2252-7

5. Vasile VC, Saenger AK, Kroning JM, Jaffe AS. Biological and analytical variability of a novel high-sensitivity cardiac troponin T assay. Clin Chem. 2010;56:1086-1090.

6. Wu A, Lu QA, Todd J, Moecks J, Wians F. Short- and long-term biological variation in cardiac troponin I measured with a high-sensitivity assay: implications for clinical practice. Clin Chem. 2009;55:52-58.

7. Keller T, Zeller T, Peetz D et al. Sensitive troponin I assay in early diagnosis of acute myocardial infarction. N Engl J Med. 2009;361:868-877.

8. Apple FS, Pearce LA, Smith SW, Kaczmarek JM, Murakami MM. Role of monitoring changes in sensitive cardiac troponin I assay results for early diagnosis of myocardial infarction and prediction of risk of adverse events. Clin Chem. 2009;55:930-937.

9. Hammarsten O, Fu ML, Sigurjonsdottir R et al. Troponin T percentiles from a random population sample, emergency room patients and patients with myocardial infarction. Clin Chem. 2012;58:628-637. 
Cardiac Troponins: State of the (He)Art 


\section{The effect of a six-month resistance-type}

exercise training program on the course of high sensitive cardiac troponin T levels in (pre)frail elderly

Noreen van der Linden*, Michael Tieland*, Lieke Klinkenberg, Lex Verdijk, Lisette de Groot, Luc van Loon, Marja van Dieijen-Visser, Steven Meex-Int J Cardiol.2014 Aug 1;175(2):374-5 * equal contribution 


\section{Introduction}

Cardiac troponin is the preferred biomarker for diagnosing non ST-segment elevation myocardial infarction ${ }^{1}$. With the introduction of high-sensitivity assays, cardiac troponin levels became also detectable in apparently healthy subjects. This has expanded its role from acute cardiac care to risk stratification and prognostic medicine ${ }^{2}$. Meanwhile, high sensitive cardiac troponin (hs-cTn) has acquired the status of a prognostic biomarker that predicts cardiovascular risk, in addition to established Framingham risk factors such as age, sex, blood pressure and dyslipidemia ${ }^{3}$. Despite the remarkable consistency in literature on the association between cardiac troponin levels and increased risk for cardiovascular morbidity and mortality, it is unclear whether preventative measures such as lifestyle intervention programs can influence the course of basal troponin levels. Suggestive evidence in favor of this hypothesis was provided by an observational study by deFilippi et al, showing that higher physical activity levels in elderly subjects were associated with both lower basal cardiac troponin $\mathrm{T}$ (cTnT) levels, and a lower probability of a significant increase in cTnT concentrations between consecutive visits ${ }^{4}$. However, no intervention study has evaluated the effect of an exercise program on the concentration of cardiac troponin. The present trial was conducted to investigate the hypothesis that a 24-week resistance-type training program modulates the course of basal plasma cTnT levels in (pre)frail elderly subjects. Coronary artery disease is common in the (pre)frail elderly population. While elderly in general are prone to cardiovascular disease, the subgroup of (pre)frail elderly is a subset of patients with highest vulnerability to adverse outcomes and all-cause mortality ${ }^{5}$. We therefore postulated that this particular patient group is characterized by high baseline levels of cTnT, and may be most receptive to the potential effects or benefits of an exercise intervention.

\section{Methods}

Elderly subjects ( $\geq 65$ years), who previously had not participated in any structured exercise training program over the past 2 years and were considered (pre)frail according to the Fried criteria $^{6}$, were eligible. Subjects diagnosed with chronic obstructive pulmonary disease 
(COPD), malignancies, muscle disease, type 2 diabetes (glucose concentrations $\geq 7 \mathrm{mmol} / \mathrm{L}$ ), renal dysfunction (eGFR $<60 \mathrm{~mL} / \mathrm{min} / 1.73 \mathrm{~m}^{2}$ ) or unable to perform the exercise regimen were excluded. The Medical Ethics Committee of Wageningen University approved this study and all subjects gave their written informed consent. Subjects participated either in a 24week supervised resistance-type exercise training program, or were followed up during a parallel non-interventional control period. Training was performed under personal supervision, and consisted of a 5-minute warming up on a cycle ergometer, 4 sets on legpress and leg-extension machines and 3 sets on chest press, lat pulldown, pecdec and vertical row machines (Technogym, Rotterdam, the Netherlands). This program was scheduled twice per week and workload was increased during the 24-week period. Blood samples were collected before and after 12 and 24 weeks of intervention. Assuming a mean baseline cTnT concentration of $11.00 \mathrm{ng} / \mathrm{L}$, a 24 -week increase of $3 \%^{4}$ (estimated baseline correlation and decay rate 0.90 and 0.25 , respectively), and an expected reduction of basal troponin levels after 24 weeks of 15\%, the inclusion of 23 subjects per group would afford $80 \%$ power at a significance level of $5 \%^{7}$. We anticipated a dropout rate of approximately $25 \%$, and therefore recruited 62 subjects for this study (31 per group). Plasma cTnT was measured using the highsensitivity cTnT assay on the Cobas-6000 analyzer (Roche Diagnostics). This study is part of a greater trial examining the impact of protein supplementation and training on skeletal muscle mass in (pre)frail elderly as primary outcome. The effects of the training program on muscle mass, muscle strength and physical performance were published recently ${ }^{8,9}$.

\section{Results}

Ten subjects withdrew from the study; 6 in the intervention group and 4 in the control group, leaving 52 subjects for follow-up analyses. Table 1 shows the clinical characteristics of the participants. All cTnT values measured during the study period (range $3.78-105.70 \mathrm{ng} / \mathrm{L}$ ) were above the detection limit of the assay ( $3 \mathrm{ng} / \mathrm{L})$, and 40 subjects (65\%) had at least one measurement above the 99th percentile of $14 \mathrm{ng} / \mathrm{L}$. Table 2 shows the baseline characteristics and the 12-week and 24-week follow-up data of both groups. Figure 1 shows the course of cTnT levels over time in both groups. Using mixed linear model analyses, we 
did not observe a significant difference in the course of basal cTnT levels between the intervention and the control group (intention-to-treat analysis $p=0.38$, per-protocol analysis $p=0.16)$.

Table 1. Clinical characteristics

\begin{tabular}{|c|c|c|}
\hline Variable & Control $(n=27)$ & Intervention $(n=25)$ \\
\hline \multicolumn{3}{|l|}{ Demographics } \\
\hline Age (years), mean (SD) & $80.2(7.4)$ & $78.6(6.8)$ \\
\hline Women, n (\%) & $14(52)$ & $17(68)$ \\
\hline BMI $\left(\mathrm{kg} / \mathrm{m}^{2}\right)$, mean (SD) & $26.6(3.3)$ & $28.7(4.9)$ \\
\hline \multicolumn{3}{|l|}{ Medical history } \\
\hline Systolic BP (mm Hg), mean (SD) & $151(24)$ & $142(18)$ \\
\hline Diastolic BP (mm Hg), mean (SD) & $75(9)$ & $72(9)$ \\
\hline Current smoking, n (\%) & $1(4)$ & $1(4)$ \\
\hline \multicolumn{3}{|l|}{ Laboratory data } \\
\hline Fasting glucose (mmol/L), mean (SD) & $5.2(0.5)$ & $5.3(0.5)$ \\
\hline Total cholesterol (mmol/L), mean (SD) & $5.4(1.1)$ & $5.2(1.5)$ \\
\hline Triglycerides (mmol/L), mean (SD) & $1.2(0.6)$ & $1.3(0.5)$ \\
\hline HDL cholesterol (mmol/L), mean (SD) & $1.5(0.4)$ & $1.3(0.3)$ \\
\hline LDL cholesterol (mmol/L), mean (SD) & $3.4(0.9)$ & $3.2(1.3)$ \\
\hline Creatinine $(\mu \mathrm{mol} / \mathrm{L})$, mean $(\mathrm{SD})$ & $77.7(12.1)$ & $74.4(14.1)$ \\
\hline eGFR $\left(\mathrm{ml} / \mathrm{min} / 1.73 \mathrm{~m}^{2}\right)$, mean (SD) & $77(11)$ & $78(13)$ \\
\hline NT-proBNP (pg/mL), median (IQR) & $19.1(8.0-46.3)$ & $14.6(6.2-25.3)$ \\
\hline \multicolumn{3}{|l|}{ Medication use } \\
\hline Antihypertensives, n (\%) & $14(52)$ & $11(44)$ \\
\hline Statins, n (\%) & $3(11)$ & $9(36)$ \\
\hline
\end{tabular}

SD, standard deviation; n, number; IQR, interquartile range; BP, blood pressure; eGFR, estimated glomerular filtration rate.

Table 2. Baseline and follow-up concentrations of cTnT

\begin{tabular}{lcc}
\hline Variable & Control $(\mathrm{n}=27)$ & Intervention $(\mathrm{n}=25)$ \\
\hline Baseline hs-cTnT (ng/L), median (IQR) & $14.7(10.0-19.7)$ & $10.3(7.8-17.5)$ \\
12-week hs-cTnT (ng/L), median (IQR) & $14.6(12.0-23.3)$ & $13.9(8.9-20.5)$ \\
24-week hs-cTnT (ng/L), median (IQR) & $14.8(11.4-25.5)$ & $14.3(8.5-20.5)$ \\
\hline
\end{tabular}




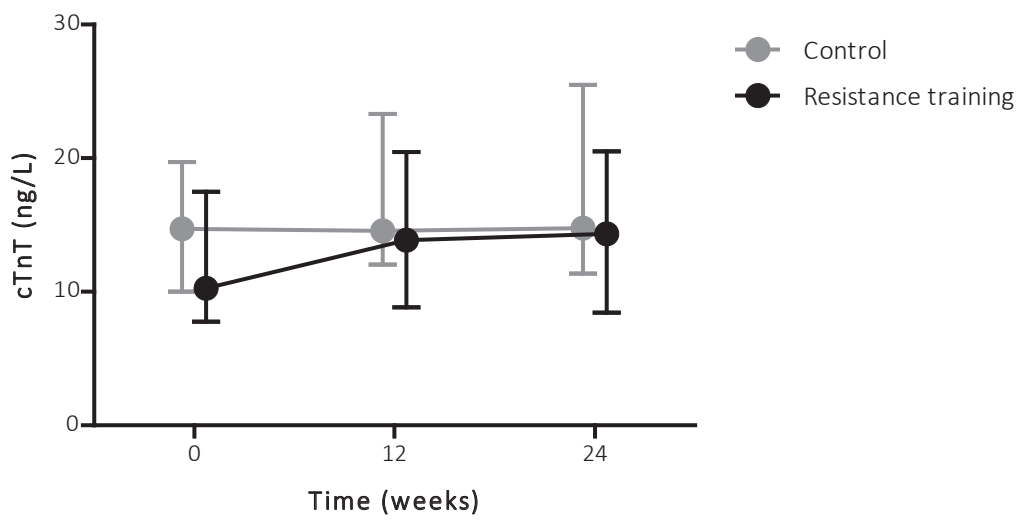

Figure 1. Median (interquartile range) concentrations of $\mathrm{cTnT}$ in the control and intervention group of (pre)frail elderly.

\section{Conclusion}

The finding that the majority of participants had cTnT levels above the 99th percentile confirms our hypothesis that chronically elevated cTnT concentrations are highly prevalent among (pre)frail elderly subjects. However, despite the elevated basal levels of cTnT and the fact that subjects had not previously participated in any structured exercise program, we found no evidence for an effect of a 24-week resistance-type exercise training program on the course of cTnT levels. A possible limitation of our study is that the exercise program was based on resistance-type exercise training, and that a more endurance-type based training may have a greater effect op cTn levels. Another limitation might have been the physical state of the subjects, which precluded a higher training frequency than the biweekly program that was applied in this study. The biweekly schedule was however sufficient to induce substantial improvements of muscle strength and physical performance in this group ${ }^{8,9}$. In conclusion, we demonstrated that prolonged resistance-type exercise training in (pre)frail elderly does not lead to changes in the course of cTnT levels. Future studies will reveal whether more intensive and more prolonged interventions can beneficially affect the course of basal troponin levels over time. 


\section{References}

1. Thygesen K, Alpert JS, Jaffe AS, et al. Third universal definition of myocardial infarction. Journal of the American College of Cardiology 2012;60:1581-98.

2. Giannitsis E, Katus HA. Cardiac troponin level elevations not related to acute coronary syndromes. Nature reviews Cardiology 2013;10:623-34.

3. Wang TJ, Wollert KC, Larson MG, et al. Prognostic utility of novel biomarkers of cardiovascular stress: the Framingham Heart Study. Circulation 2012;126:1596-604.

4. deFilippi CR, de Lemos JA, Tkaczuk AT, et al. Physical activity, change in biomarkers of myocardial stress and injury, and subsequent heart failure risk in older adults. Journal of the American College of Cardiology 2012;60:2539-47.

5. Afilalo J, Karunananthan S, Eisenberg MJ, Alexander KP, Bergman H. Role of frailty in patients with cardiovascular disease. The American journal of cardiology 2009;103:1616-21.

6. Fried LP, Tangen CM, Walston J, et al. Frailty in older adults: evidence for a phenotype. The journals of gerontology Series A, Biological sciences and medical sciences 2001;56:M146-56.

7. Guo Y, Logan HL, Glueck DH, Muller KE. Selecting a sample size for studies with repeated measures. BMC medical research methodology 2013;13:100.

8. Tieland M, Dirks ML, van der Zwaluw N, et al. Protein supplementation increases muscle mass gain during prolonged resistance-type exercise training in frail elderly people: a randomized, double-blind, placebo-controlled trial. Journal of the American Medical Directors Association 2012;13:713-9.

9. Tieland M, van de Rest O, Dirks ML, et al. Protein supplementation improves physical performance in frail elderly people: a randomized, double-blind, placebo-controlled trial. Journal of the American Medical Directors Association 2012;13:720-6. 


\section{The effect of exercise training on the}

\section{course of cardiac troponin $T$ and llevels:}

\section{three independent training studies}

Noreen van der Linden, Lieke Klinkenberg, Marika Leenders, Michael Tieland, Lex Verdijk, Marijke Niens, Jeroen van Suijlen, Lisette de Groot, Otto Bekers, Luc van Loon, Marja van Dieijen-Visser, Steven Meex - Sci Rep. 2015 Dec 16;:18320 


\section{Abstract}

With the introduction of high-sensitive assays, cardiac troponins became potential biomarkers for risk stratification and prognostic medicine. Observational studies have reported an inverse association between physical activity and basal cardiac troponin levels. However, causality has never been demonstrated. This study investigated whether basal cardiac troponin concentrations are receptive to lifestyle interventions such as exercise training. Basal high-sensitive cardiac troponin T (cTnT) and I (cTnl) were monitored in two resistance-type exercise training programs (12-week (study 1) and 24week (study 2)) in older adults ( $\geq 65$ years). In addition, a retrospective analysis for high sensitive troponin I in a 24-week exercise controlled trial in (pre)frail older adults was performed (study 3). In total, 91 subjects were included in the final data analyses. There were no significant changes in cardiac troponin levels over time in study 1 and 2 (study 1: cTnT -0.13 (-0.33-+0.08) ng/L/12-weeks, cTnl $0.10(-0.33-+0.12) \mathrm{ng} / \mathrm{L} / 12$-weeks; study 2: CTnT -1.99 (-4.79-+0.81) ng/L/24-weeks, cTnl -1.59 (-5.70 $-+2.51) \mathrm{ng} / \mathrm{L} / 24$-weeks). Neither was there a significant interaction between training and the course of cardiac troponin in study $3(p=0.27)$. In conclusion, this study provides no evidence that prolonged resistance-type exercise training can modulate basal cardiac troponin levels. 


\section{Introduction}

The development of increasingly sensitive assays for cardiac troponin has now reached the point where cardiac troponin concentrations can be accurately assessed in the majority of subjects from a healthy reference population ${ }^{1}$. Parallel to the development of assays with increasing sensitivity, interest in cardiac troponin has expanded from acute cardiac care to risk prediction and risk stratification ${ }^{2}$. Studies in various patient groups and asymptomatic individuals have provided compelling evidence that baseline cardiac troponin levels predict outcome $^{3}$. Interestingly, the observed risk gradient is not restricted to elevations above the $99^{\text {th }}$ percentile, but is even apparent within the 'healthy' population reference interval, and independent of traditional risk factors such as age, sex and diabetes ${ }^{4-9}$. In the Framingham heart study, the basal cardiac troponin I concentration adds prognostic value to standard risk factors for predicting death, and cardiovascular disease ${ }^{10}$. Furthermore, temporal increases in cardiac troponin concentrations over time confer additional cardiovascular risk, and are inversely related to the level of physical fitness ${ }^{7,11}$. These observations form the basis of the hypothesis that cardiac troponin is a modifiable parameter, which may be receptive to lifestyle interventions such as an exercise training program. In a recent study with (pre)frail subjects we tested the hypothesis that a supervised resistance-type exercise training program can influence the course of cardiac troponin levels over time ${ }^{12}$. However, this $24-$ week supervised bi-weekly exercise training program conferred no beneficial effect on the course of cardiac troponin T levels, despite substantial improvements of these subjects at the level of physical performance ${ }^{12}$. This negative study was conducted in frail and pre-frail subjects, characterized by substantially elevated baseline cardiac troponin levels, and therefore theoretically most amenable to the potential benefits of an exercise intervention. A possible drawback however, was the limited training load that could be imposed due to the level of frailty of these older subjects. Nevertheless, despite these limitations, our results were similar to those of other recent reports: 14-week endurance training in untrained adults $^{13}$, 17-week half-marathon training in previously sedentary men ${ }^{14}$ and 3-month exercise training in heart failure patients (NYHA class II-IV) ${ }^{15}$ did not result reduce basal cardiac troponin T concentrations. However, limitations of these studies were the low basal 
cardiac troponin concentrations ${ }^{13}$ and the use of conventional instead of high-sensitive cardiac troponin T assays ${ }^{14,15}$ which reduced the power to detect an effect. Another issue that merits attention is that the effect of training intervention on cardiac troponin I has never been assessed. Since cardiac troponin $\mathrm{T}$ and I are two different proteins of the cardiac troponin complex with different biochemical characteristics ${ }^{16}$ and distinct release patterns ${ }^{17}$, both cardiac troponins may also respond differently to lifestyle interventions.

The aim of the current study is 1) to overcome the limitations of previous studies, and 2) to examine the effect of exercise training on basal cardiac troponin I levels. Therefore, we now present two additional exercise training studies to the effect of a more intense training regimen on the course of high-sensitive cardiac troponin $T$ and I levels in older adults, a population that often exhibits elevated basal cardiac troponin concentrations. Additional high sensitive troponin I was measured in our previously conducted study in (pre)frail subjects in order to present a complete and comprehensive overview to the effects of three supervised training programs.

\section{Methods}

To investigate the effect of resistance-type exercise training on cardiac troponin T (cTnT) and I (cTnl) in older adults, two independent studies were conducted: a study to the effect of 12week resistance-type exercise training in older men ( $\geq 65$ years) (study 1 ) and a second study to the effect of a 24-week resistance-type exercise training older men and women $(\geq 65$ years) (study 2). In addition, we performed a retrospective analysis for cardiac troponin I in our recently published study to the effect of a 24-week resistance-type exercise training program on the course of troponin T levels in (pre)frail subjects ( $\geq 65$ years) (study 3 ).

All studies complied with the principles of the Declaration of Helsinki and were approved by the local institutional review boards and the ethics committees of Wageningen University or Maastricht University Medical Center. Participants were recruited by advertisements in local newspapers (study 1 and 2) or by approaching elderly living in apartment houses and in homes for the elderly (study 3 ). All participants provided written informed consent. Subjects 
were only eligible for inclusion when they had not participated in any structured exercise training program over the past 2 years.

\section{Study 1: 12-week training in older men}

In total 14 subjects were included in study 1 . Inclusion criteria were age (65 - 85 years), male gender and the ability to understand and perform study procedures. Exclusion criteria were (silent) cardiac or peripheral vascular disease, orthopedic limitations (self-reported) and type 2 diabetes (based on an oral glucose tolerance test). A training session consisted of a 5minute warming up on a cycle ergometer, four sets on leg-press and leg-extension machines and a 5-minute cooling down on the cycle ergometer. The workload started at $60 \%$ of 1 repetition maximum (1-RM) (10-15 repetitions per set) and was increased to $75-80 \%$ of 1 RM (8-10 repetitions per set). The typical duration of a training session was approximately 45 minutes, and the work:rest ratio was 1:4. This supervised resistance-type exercise training program was performed three times per week during 12 weeks. There was at least a resting period of 2 days between consecutive training sessions. Blood samples were obtained by a venipuncture after an overnight fast, at least three days after the last training session, before the start of the training program (week 0) and after 4, 8 and 12 weeks of intervention. This study is a substudy of a more extensive trial examining the effects of resistance-type exercise training in older men on muscle strength, body composition and muscle fiber type-specific characteristics as primary outcome ${ }^{18}$.

\section{Study 2: 24-week training in older men and women}

In total 27 subjects were included in study 2. Inclusion criteria were age (65-90 years) and the ability to understand and perform study procedures. Exclusion criteria were the same as in study 1. Training sessions consisted of a 5-minute warming up on a cycle ergometer, four sets on leg-press and leg-extension machines and three sets on the chest press and horizontal row; these four exercises were performed every training session. In addition, three sets of vertical lat pull and abdominal crunches were alternated with biceps curl and triceps extension between subsequent training sessions. The workload started at 60\% of 1-RM (10- 
15 repetitions per set) and was increased to $75-80 \%$ of 1-RM (8-10 repetitions per set). The typical duration of a training session was approximately 45 minutes, and the work:rest ratio was 1:4. This supervised resistance-type exercise training program was performed three times per week during 24 weeks. There was at least a resting period of 2 days between consecutive training sessions. Blood samples were obtained by a venipuncture after an overnight fast at least three days after the last training session before the start of the training program (week 0) and after 4, 8, 12, 16, 20 and 24 weeks of intervention. This is a substudy of a more extensive trial to the effects of an exercise training intervention in older adults on muscle strength, body composition and muscle fiber type-specific characteristics. In this current study we included only subjects from the control group who were not receiving dietary protein supplementation (as described in the original study protocol) ${ }^{19,20}$.

\section{Study 3: 24-week training in (pre)frail older men and women}

Methodological details of study 3 have been described previously ${ }^{12}$. Briefly, a total of 62 (pre)frail older men and women ( $\geq 65$ years) were included and were equally distributed in an intervention and a control group. Frailty and prefrailty were defined according to the Fried criteria (subjects were considered prefrail when 1 or 2 criteria were applicable, and frail when 3 or more criteria were present): (1) unintentional weight loss, (2) weakness, (3) selfreported exhaustion, (4) slow walking speed, and (5) low physical activity ${ }^{21}$. The intervention group participated in a 24-week, biweekly, supervised resistance-type exercise training program. Blood samples were obtained by a venipuncture after an overnight fast before the start of the training program (week 0), after 12 and after 24 weeks of intervention. The effect of the training program on muscle mass, muscle strength, physical performance, and troponin T levels were published recently. In this current study we included only subjects from the control group who were not receiving dietary protein supplementation (as described in the original study protocol) ${ }^{12,22,23}$. 


\section{Cardiac troponin T and I}

Plasma and serum samples were stored at $-80^{\circ} \mathrm{C}$ until analysis. Serum (study 1 ) and plasma (study 2 and 3) cardiac troponins were measured using the high sensitive cTnT assay (limit of blank $3.0 \mathrm{ng} / \mathrm{L}$, limit of detection $5.0 \mathrm{ng} / \mathrm{L}$, 99th percentile $14 \mathrm{ng} / \mathrm{L}^{24}$ ) on the Cobas analyzer (Roche Diagnostics) and the high sensitive cTnl assay (according to the manufacturer, limit of blank $0.7-1.3 \mathrm{ng} / \mathrm{L}$, limit of detection $1.1-1.9 \mathrm{ng} / \mathrm{L}, 99^{\text {th }}$ percentile $26.2 \mathrm{ng} / \mathrm{L}$ and $10 \% \mathrm{CV}$ $4.7 \mathrm{ng} / \mathrm{L}$ ) on the ARCHITECT analyzer (Abbott Diagnostics).

\section{Statistical analysis}

Normally distributed parameters were expressed as mean \pm standard deviation (SD), nonnormally distributed parameters as median and interquartile range (IQR), and categorical variables as number $(\mathrm{n})$ and percentage (\%). Changes in basal cardiac troponin $\mathrm{T}$ and I concentrations over time were analyzed using mixed linear model analyses (random intercept) with the covariate time as fixed effect. The inclusion of 14 subjects in study 1 and 27 subjects in study 2 afforded at least 90\% power at a significance level of 5\% to detect a $10 \%$ reduction in cardiac troponin levels, anticipating a dropout rate of $25 \%$. These post-hoc power calculations were based on the assumption of a mean baseline cTnT concentration of $8.0 \mathrm{ng} / \mathrm{L}$ and $\mathrm{CTnl}$ concentration of $6.0 \mathrm{ng} / \mathrm{L}$ for study 1 and a mean baseline cardiac CTnT concentration of $6.0 \mathrm{ng} / \mathrm{L}$ and a cTnl concentration of $4.0 \mathrm{ng} / \mathrm{L}$ for study 2 with older men and women (estimated baseline correlation and decay rate 0.90 and 0.25 , respectively) ${ }^{25}$. We used two-level design mixed linear model analyses (random intercept) with time and intervention as fixed effects to assess the differences in the course of cardiac troponin I between (pre)frail subjects in the control group and in the intervention group. Power calculation for study 3 has been described previously ${ }^{12}$. All statistical analyses were performed using SPSS, version 20.0. P $\leq 0.05$ was considered statistically significant for all analyses. 


\section{Results}

\section{Compliance to the training program}

Included subjects in study 1 and 2 demonstrated $97 \%$ and $90 \%$ compliance, respectively, to the scheduled, supervised training sessions. In each study one subject withdrew. Ten subjects (15\%) withdrew from study 3. Subjects withdrew because of time constraints and medical reasons unrelated to the exercise training. In total, in the final data analysis we included: 13 older men participating in the 12-week training program (study 1), 26 older men and women participating in the 24-week training program (study 2), and 52 subjects in the retrospective troponin I analysis with (pre)frail older men and women (study 3). Table 1 shows the subjects' clinical characteristics at baseline. Baseline characteristics for the (pre)frail subjects have been published previously ${ }^{12}$.

\section{No decrease in cardiac troponin T and I levels during the exercise training program}

Fig. 1 shows the course of cardiac troponin T and cardiac troponin I levels over time in study 1 and 2 (for individual data see Supplementary Fig. S1 and S2). Table 2 shows the corresponding baseline and the follow-up values for cTnT and cTnl. One subject in study 2 had cardiac troponin T concentrations below the limit of blank ( $3 \mathrm{ng} / \mathrm{L}$ ) at all time points, and was therefore excluded from the cardiac troponin T analysis. $98.6 \%$ and $99.6 \%$ of all other troponin $\mathrm{T}$ and I measurements respectively had concentrations above the limit of blank. Using mixed linear model analyses, we found no significant changes in cardiac troponin levels over time in older men participating in a 12-week resistance-type exercise training program (cTnT-0.13 (-0.33-+0.08) ng/L/12-weeks ( $p=0.16), c T n l-0.10(-0.33-+0.12)$ ng/L/12-weeks $(p=0.44))$ and in older men and women participating in a 24-week resistance-type exercise training program (cTnT -1.99 $(-4.79-+0.81)$ ng/L/24-weeks $(p=0.23)$, cTnl -1.59 (-5.70 +2.51) ng/L/24-weeks ( $p=0.37))$. 


\begin{tabular}{|c|c|c|c|c|}
\hline & $\begin{array}{c}\text { Older men } \\
12-w \text { intervention } \\
(n=13)\end{array}$ & $\begin{array}{c}\text { Older men and } \\
\text { women } \\
\text { 24-w intervention } \\
(n=26)\end{array}$ & $\begin{array}{l}\text { (pre)Frail older } \\
\text { men and women } \\
24-w \text { intervention } \\
\quad(n=25)\end{array}$ & $\begin{array}{l}\text { (pre)Frail older } \\
\text { men and women } \\
\text { control } \\
(n=27)\end{array}$ \\
\hline Age (years), mean (SD) & $72.4(5.4)$ & $69.2(4.1)$ & $78.6(6.8)$ & $80.2(7.4)$ \\
\hline Women, n (\%) & $0(0)$ & $12(46)$ & $17(68)$ & $14(52)$ \\
\hline BMI $\left(\mathrm{kg} / \mathrm{m}^{2}\right)$, mean (SD) & $27.4(3.8)$ & $25.9(2.0)$ & 28.7 (4.9) & $26.6(3.3)$ \\
\hline \multicolumn{5}{|l|}{ Medical history } \\
\hline $\begin{array}{l}\text { Systolic BP (mm Hg), } \\
\text { mean (SD) }\end{array}$ & $147(14)$ & $137(15)$ & $142(18)$ & $151(24)$ \\
\hline $\begin{array}{l}\text { Diastolic BP (mm Hg), } \\
\text { mean (SD) }\end{array}$ & $82(17)$ & $77(22)$ & $72(9)$ & 75 (9) \\
\hline \multicolumn{5}{|l|}{ Laboratory data } \\
\hline $\begin{array}{l}\text { Fasting glucose } \\
(\mathrm{mmol} / \mathrm{L}) \text {, mean (SD) }\end{array}$ & $5.6(0.7)$ & $5.5(0.5)$ & $5.3(0.5)$ & $5.2(0.5)$ \\
\hline $\begin{array}{l}\text { Total cholesterol } \\
\text { (mmol/L), mean (SD) }\end{array}$ & $5.6(1.1)$ & $6.2(0.9)$ & $5.2(1.5)$ & $5.4(1.1)$ \\
\hline $\begin{array}{l}\text { Triglycerides (mmol/L), } \\
\text { mean (SD) }\end{array}$ & $1.4(0.5)$ & $1.3(0.5)$ & $1.3(0.5)$ & $1.2(0.6)$ \\
\hline $\begin{array}{l}\text { HDL cholesterol } \\
(\mathrm{mmol} / \mathrm{L}) \text {, mean }(\mathrm{SD})\end{array}$ & $1.3(0.4)$ & $1.6(0.3)$ & $1.3(0.3)$ & $1.5(0.4)$ \\
\hline $\begin{array}{l}\text { LDL cholesterol } \\
\text { (mmol/L), mean (SD) }\end{array}$ & $3.6(1.3)$ & $4.3(0.8)$ & $3.2(1.3)$ & $3.4(0.9)$ \\
\hline $\begin{array}{l}\text { Creatinine }(\mu \mathrm{mol} / \mathrm{L}) \text {, } \\
\text { mean }(\mathrm{SD})\end{array}$ & $102.2(7.5)$ & $88.1(17.4)$ & $74.4(14.1)$ & $77.7(12.1)$ \\
\hline $\begin{array}{l}\text { Cystatin C (mg/L), mean } \\
\text { (SD) }\end{array}$ & $1.07(0.11)$ & $0.90(0.19)$ & $1.00(0.29)$ & $1.08(0.25)$ \\
\hline $\begin{array}{l}\text { eGFR }\left(\mathrm{ml} / \mathrm{min} / 1.73 \mathrm{~m}^{2}\right) \\
\text { mean }(\mathrm{SD})^{*}\end{array}$ & $66.3(7.2)$ & $78.3(15.2)$ & $73.9(15.4)$ & $69.6(13.9)$ \\
\hline $\begin{array}{l}\text { NT-proBNP }(\mathrm{pg} / \mathrm{mL}) \text {, } \\
\text { median (IQR) }\end{array}$ & $12.1(6.8-21.0)$ & $7.6(3.1-15.8)$ & $14.6(6.2-25.3)$ & $19.1(8.0-46.3)$ \\
\hline \multicolumn{5}{|l|}{ Medication use } \\
\hline Antihypertensives, n (\%) & $3(25)$ & $3(12)$ & $11(44)$ & $14(52)$ \\
\hline Statins, n (\%) & $1(8)$ & $1(4)$ & $9(36)$ & $3(11)$ \\
\hline
\end{tabular}


A

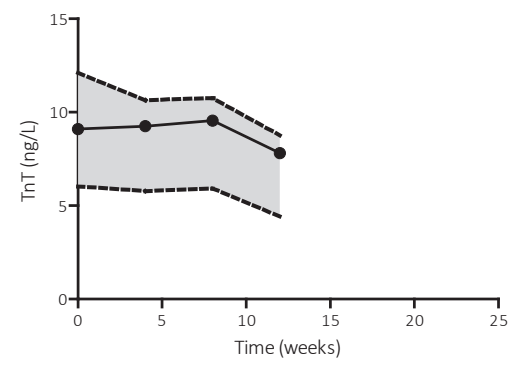

C

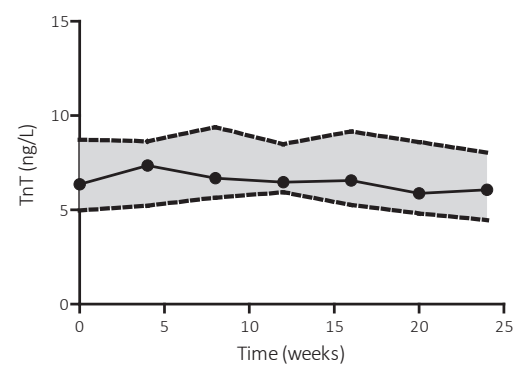

B

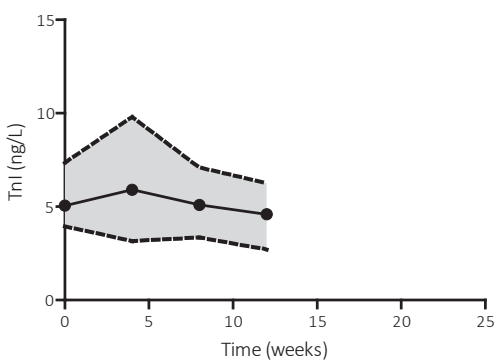

D

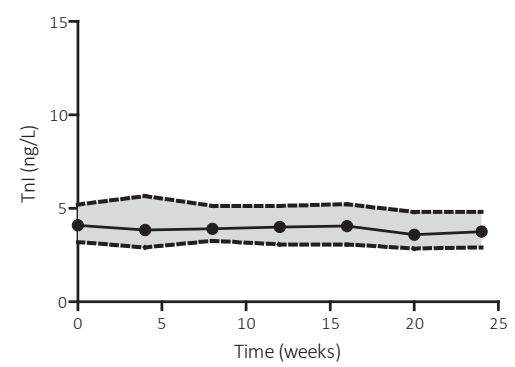

Fig. 1. Course of cardiac troponinT and $\mathrm{I}$ in older adults participating in 12- and 24-week training programs. Median (interquartile range) concentrations of cTn in older adults participating in a supervised resistance-type training program: A, course of cTnT in older men in a 12-week training program; $\mathrm{B}$, course of cTnl in older men in a 12-week training program; C, course of cTnT in older men and women in a 24-week training program; D, course of $\mathrm{cTnl}$ in older men and women in a 24-week training program. 
Table 2. Baseline and follow-up concentrations of cTn in older adults participating in 12-week and 24-week training programs

\begin{tabular}{lcccc}
\hline & \multicolumn{2}{c}{ hs-cTnT (ng/L) , median (IQR) } & \multicolumn{2}{c}{ hs-cTnl (ng/L), median (IQR) } \\
\hline & $\begin{array}{cccc}\text { 12-w intervention } \\
(n=13)\end{array}$ & $\begin{array}{c}24-w \text { intervention } \\
(n=25)\end{array}$ & $\begin{array}{c}\text { 12-w intervention } \\
(n=13)\end{array}$ & 24-w intervention \\
Baseline & $9.1(6.0-12.1)$ & $6.4(5.0-8.7)$ & $5.1(4.0-7.4)$ & $4.1(3.2-5.2)$ \\
Week 4 & $9.3(5.8-10.7)$ & $7.4(5.2-8.6)$ & $5.9(3.2-9.8)$ & $3.9(2.9-5.7)$ \\
Week 8 & $9.6(5.9-10.8)$ & $6.7(5.6-9.4)$ & $5.1(3.4-7.1)$ & $3.9(3.3-5.1)$ \\
Week 12 & $7.8(4.4-8.8)$ & $6.5(5.9-8.5)$ & $4.6(2.7-6.3)$ & $4.0(3.1-5.1)$ \\
Week 16 & & $6.6(5.3-9.2)$ & & $4.1(3.1-5.2)$ \\
Week 20 & & $5.9(4.8-8.6)$ & & $3.6(2.9-4.8)$ \\
Week 24 & & $6.1(4.5-8.0)$ & & $3.8(2.9-4.8)$ \\
\hline
\end{tabular}

Table 3. Baseline and follow-up concentrations of hs-cTnl in (pre)frail older subjects in the control group and those participating in a 24-week resistance-type exercise training program

\begin{tabular}{lcc}
\hline & $\begin{array}{c}\text { (pre)Frail older subjects 24-w } \\
\text { intervention } \\
(\mathrm{n}=25)\end{array}$ & $\begin{array}{c}\text { (pre)Frail older subjects } \\
\text { no intervention } \\
(\mathrm{n}=27)\end{array}$ \\
\hline Baseline hs-cTnl (ng/L), median (IQR) & $4.4(3.3-6.2)$ & $6.7(4.7-7.7)$ \\
12-week hs-cTnl (ng/L), median (IQR) & $4.6(3.5-6.4)$ & $6.7(4.4-8.4)$ \\
24-week hs-cTnl (ng/L), median (IQR) & $4.3(3.6-8.4)$ & $6.0(3.4-7.6)$ \\
\hline
\end{tabular}

\section{No effect of a 24-week resistance-type exercise training program on cardiac troponin I levels}

Table 3 shows the baseline cTnl concentrations and the 12-week and 24-week follow-up data of (pre)frail older men and women. In the control group, all values were above the limit of blank of the assay $(1.3 \mathrm{ng} / \mathrm{L}), 3$ subjects had at least one value above the $99^{\text {th }}$ percentile of $26.2 \mathrm{ng} / \mathrm{L}$, which is used for the diagnosis of myocardial infarction. In the intervention group, one subject had one value below limit of blank of the assay, and one subject had at least one value above the $99^{\text {th }}$ percentile. Mixed linear model analyses revealed no significant interaction between training and the course of cTnl levels between the intervention and the control group (intention-to-treat analysis $p=0.27$, per-protocol analysis $p=0.27$ ) (for individual data see Supplementary Fig. S2). 


\section{Discussion}

In two independent supervised training studies of 12 and 24 weeks respectively, we found no evidence for a modifying effect of resistance-type exercise training on the course of cardiac troponin T and I levels in older subjects, nor did we find an effect of a 24-week training program on cardiac troponin I levels in (pre)frail subjects.

Our results do not support the idea that cardiac troponin levels might be modifiable through exercise. This hypothesis was postulated by observational studies, showing that higher physical activity levels in older subjects were associated with both lower basal cTnT levels, and a lower probability of a significant increase in cTnT concentrations between consecutive visits $^{11,26}$. To investigate whether a causal relationship underlies this association, we conducted a series of training studies in subjects where stable elevated cardiac troponin levels are common, and who are therefore - at least theoretically - most receptive to the favorable effects of an intervention. To ensure that basal cardiac troponin levels, rather than acute post-exercise effects were studied, we included a three day interval between the last training session and the blood sampling procedure ${ }^{27}$. None of these studies showed a favorable effect of resistance-type exercise training on cardiac troponin levels. The nonresponsive pattern of troponin T and I to an exercise program is in contrast with the beneficial changes observed for leg strength, muscle mass, insulin sensitivity, $\mathrm{HbA1c}$, total cholesterol and $\operatorname{LDL}^{18-20,22,23}$. The present results confirm and extend the results of our previous training intervention study in (pre)frail older adults ${ }^{12}$, which was also characterized by favorable effects on physical performance, but a lack of effect on circulating cardiac troponin levels. Although the absolute number of subjects in these training studies are relatively low, all studies afforded at least $90 \%$ power to detect a $10 \%$ reduction of cardiac troponin over time. We cannot exclude the possibility that training confers a smaller effect on the course of troponin levels that goes by undetected in the present study, but we feel that (much) smaller changes than specified in our power calculation would comprise limited clinical relevance. A possible limitation of our study relates to the type of exercise training intervention, comprising mostly resistance-type exercise. However the subjects' physical state precluded (intense) endurance training. Despite the physical and metabolic improvements found in our 
studies $^{18-20,22,23}$, a valid question may be whether resistance-type exercise training is the most appropriate type of training to generate a favorable effect on cardiac troponin concentrations. In this respect the results of a recently conducted cardio-based exercise training intervention study in heart failure patients are interesting. Similar to our studies, no favorable effect was found of a supervised endurance exercise training program on cardiac troponin T levels ${ }^{15}$.

The supervision during the sessions contributed to the internal validity, but might also have led to increased safety, less adverse events, and a higher motivation among subjects. This may overestimate any beneficial effects of exercise training. Another point regarding the validity of our study is the participant recruitment process and study design. Since participation in the study is associated with a substantial time investment and a relatively high intensity training program, this might have attracted a specific, highly motivated subpopulation. Since our study was conducted in an elderly population we can only speculate about the effects of exercise training on basal cardiac troponin levels in younger subjects who may respond differently on exercise training and demonstrate higher cardiovascular plasticity ${ }^{28}$. Nevertheless, we show that 12 and 24 weeks of supervised resistance-based exercise training does not reduce basal cardiac troponin $T$ and I concentrations in older adults. 


\section{References}

1. Koerbin G, Tate J, Potter JM, Cavanaugh J, Glasgow N, Hickman PE. Characterisation of a highly sensitive troponin I assay and its application to a cardio-healthy population. Clinical chemistry and laboratory medicine : CCLM / FESCC 2012;50:871-8.

2. Giannitsis E, Katus HA. Highly sensitive troponins knocking at the door of primary prevention. European heart journal 2014;35:268-70.

3. Mills NL, Churchhouse AM, Lee KK, et al. Implementation of a sensitive troponin I assay and risk of recurrent myocardial infarction and death in patients with suspected acute coronary syndrome. JAMA : the journal of the American Medical Association 2011;305:1210-6.

4. de Lemos JA, Drazner MH, Omland T, et al. Association of troponin T detected with a highly sensitive assay and cardiac structure and mortality risk in the general population. Jama 2010;304:2503-12.

5. Saunders JT, Nambi V, de Lemos JA, et al. Cardiac troponin T measured by a highly sensitive assay predicts coronary heart disease, heart failure, and mortality in the Atherosclerosis Risk in Communities Study. Circulation 2011;123:1367-76.

6. Zeller T, Tunstall-Pedoe H, Saarela O, et al. High population prevalence of cardiac troponin I measured by a highsensitivity assay and cardiovascular risk estimation: the MORGAM Biomarker Project Scottish Cohort. European heart journal 2014;35:271-81.

7. Eggers KM, Venge P, Lindahl B, Lind L. Cardiac troponin I levels measured with a high-sensitive assay increase over time and are strong predictors of mortality in an elderly population. Journal of the American College of Cardiology 2013;61:1906-13.

8. White HD, Tonkin A, Simes J, et al. Association of contemporary sensitive troponin I levels at baseline and change at 1 year with long-term coronary events following myocardial infarction or unstable angina: results from the LIPID Study (Long-Term Intervention With Pravastatin in Ischaemic Disease). Journal of the American College of Cardiology 2014;63:345-54.

9. Collinson P. The role of cardiac biomarkers in cardiovascular disease risk assessment. Current opinion in cardiology 2014.

10. Wang TJ, Wollert KC, Larson MG, et al. Prognostic utility of novel biomarkers of cardiovascular stress: the Framingham Heart Study. Circulation 2012;126:1596-604.

11. deFilippi CR, de Lemos JA, Tkaczuk AT, et al. Physical activity, change in biomarkers of myocardial stress and injury, and subsequent heart failure risk in older adults. Journal of the American College of Cardiology 2012;60:253947.

12. van der Linden $\mathrm{N}$, Tieland $\mathrm{M}$, Klinkenberg $\mathrm{L}$, et al. The effect of a six-month resistance-type exercise training program on the course of high sensitive cardiac troponin T levels in (pre)frail elderly. International journal of cardiology 2014;175:374-5.

13. Legaz-Arrese A, Lopez-Laval I, George K, et al. Impact of an endurance training program on exercise-induced cardiac biomarker release. Am J Physiol Heart Circ Physiol 2015;308:H913-20.

14. Vance DD, Chen GL, Stoutenberg M, et al. Cardiac performance, biomarkers and gene expression studies in previously sedentary men participating in half-marathon training. BMC sports science, medicine and rehabilitation 2014;6:6.

15. Ahmad T, Fiuzat M, Mark DB, et al. The effects of exercise on cardiovascular biomarkers in patients with chronic heart failure. American heart journal 2014;167:193-202 e1.

16. Katrukha IA. Human cardiac troponin complex. Structure and functions. Biochemistry (Mosc) 2013;78:1447-65.

17. Agewall S, Giannitsis E, Jernberg T, Katus H. Troponin elevation in coronary vs. non-coronary disease. European heart journal 2011;32:404-11.

18. Verdijk LB, Gleeson BG, Jonkers RA, et al. Skeletal muscle hypertrophy following resistance training is accompanied by a fiber type-specific increase in satellite cell content in elderly men. The journals of gerontology Series A, Biological sciences and medical sciences 2009;64:332-9. 
19. Leenders M, Verdijk LB, van der Hoeven L, van Kranenburg J, Nilwik R, van Loon LJ. Elderly men and women benefit equally from prolonged resistance-type exercise training. The journals of gerontology Series $A$, Biological sciences and medical sciences 2013;68:769-79.

20. Leenders M, Verdijk LB, Van der Hoeven L, et al. Protein supplementation during resistance-type exercise training in the elderly. Medicine and science in sports and exercise 2013;45:542-52.

21. Fried LP, Tangen CM, Walston J, et al. Frailty in older adults: evidence for a phenotype. The journals of gerontology Series A, Biological sciences and medical sciences 2001;56:M146-56.

22. Tieland $M$, Dirks ML, van der Zwaluw N, et al. Protein supplementation increases muscle mass gain during prolonged resistance-type exercise training in frail elderly people: a randomized, double-blind, placebo-controlled trial. Journal of the American Medical Directors Association 2012;13:713-9.

23. Tieland $M$, van de Rest O, Dirks ML, et al. Protein supplementation improves physical performance in frail elderly people: a randomized, double-blind, placebo-controlled trial. Journal of the American Medical Directors Association 2012;13:720-6.

24. Giannitsis E, Kurz K, Hallermayer K, Jarausch J, Jaffe AS, Katus HA. Analytical validation of a high-sensitivity cardiac troponin T assay. Clinical chemistry 2010;56:254-61.

25. Guo Y, Logan HL, Glueck DH, Muller KE. Selecting a sample size for studies with repeated measures. BMC medical research methodology 2013;13:100.

26. Klenk J, Denkinger M, Nikolaus T, et al. Association of objectively measured physical activity with established and novel cardiovascular biomarkers in elderly subjects: every step counts. Journal of epidemiology and community health 2013;67:194-7.

27. Savukoski T, Mehtala L, Lindahl B, Venge P, Pettersson K. Elevation of cardiac troponins measured after recreational resistance training. Clin Biochem 2015.

28. Wang E, Naess MS, Hoff J, et al. Exercise-training-induced changes in metabolic capacity with age: the role of central cardiovascular plasticity. Age (Dordr) 2014;36:665-76.

29. Inker LA, Schmid CH, Tighiouart $H$, et al. Estimating glomerular filtration rate from serum creatinine and cystatin

C. The New England journal of medicine 2012;367:20-9. 


\section{Supplemental information}

\section{Supplemental Figures}

12-week training older men

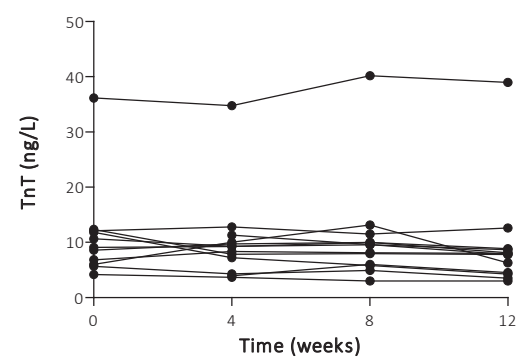

Supplementary Figure 1. Individual troponin T profiles

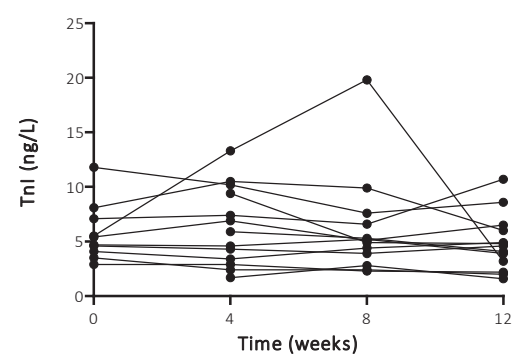

24-week training (pre)frail older men and women

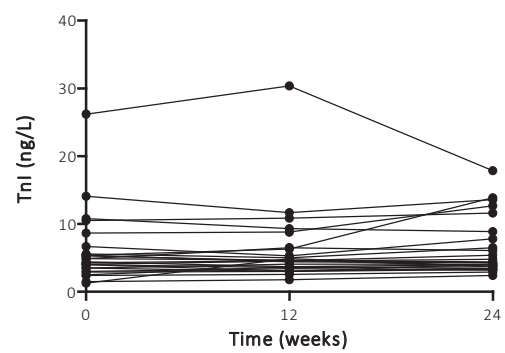

24-week training older men and women

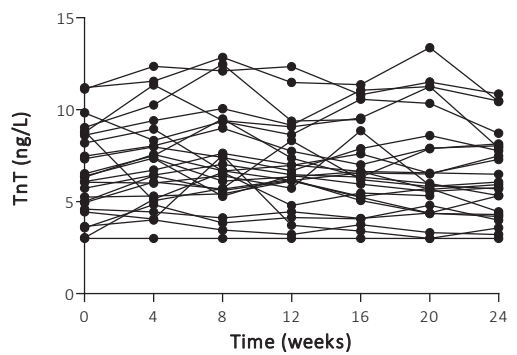

24-week training older men and women

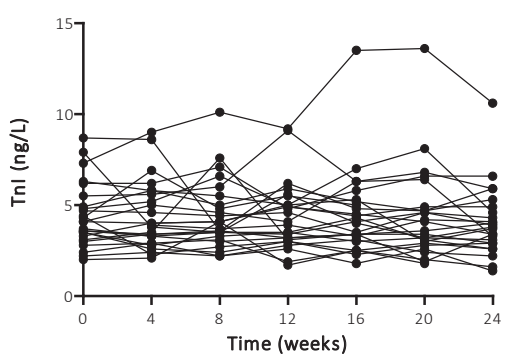

24-week control (pre)frail older men and women

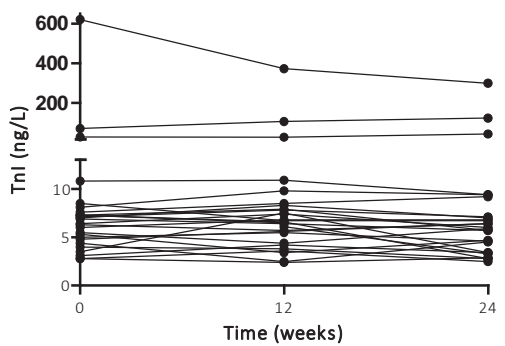

Supplementary Figure 2. Individual troponin I profiles 


\section{Prognostic value of basal high-sensitive}

\section{cardiac troponin levels on mortality in the general population: a meta-analysis}

Noreen van der Linden, Lieke Klinkenberg, Otto Bekers, Luc van Loon, Marja van Diejjen-Visser, Maurice Zeegers, Steven Meex-Medicine (Baltimore). 2016 Dec;95(52): 5703 


\begin{abstract}
Background

Interest in the use of cardiac troponin T (cTnT) and cardiac troponin I (cTnl) has expanded from diagnosis of acute myocardial infarction to risk assessment for morbidity and mortality. Although cTnT and $\mathrm{cTnI}$ were shown to have equivalent diagnostic performance in the setting of suspected acute myocardial infarction, potential prognostic differences are largely unexplored. The aim of this study is to quantify and compare the relationship between cTnT and cTnl, and cardiovascular and all-cause mortality in the general population.
\end{abstract}

\title{
Methods
}

Medline, Embase and the Cochrane Library (from inception through October 2016) were searched for prospective observational cohort studies reporting on the prognostic value of basal high-sensitive cTnT and/or cTnl levels on cardiovascular and all-cause mortality in the general population. Data on study characteristics, participants' characteristics, outcome parameters and quality (according to the Effective Public Health Practice Project (EPHPP) 'Quality Assessment Tool For Quantitative Studies') were retrieved. Hazard ratios per standard deviation increase in basal cardiac troponin level (HR per 1SD; retrieved from the included articles or estimated) were pooled using a random-effects model.

\section{Results}

On a total of 2585 reviewed citations, 11 studies, with data on 65019 participants, were included in the meta-analysis. Random effects pooling showed significant associations between basal cardiac troponin levels and hazard ratio for cardiovascular and all-cause mortality (HR per 1-SD 1.29 [95\% Cl, 1.20 to 1.38] and HR per 1-SD 1.18 [95\% Cl, 1.11 to 1.26], respectively). Stratified analyses showed higher hazard ratios for CTnT than cTnl (cardiovascular mortality: CTnT HR per 1-SD 1.37 [Cl, 1.23-to-1.52]; and CTnI HR per 1-SD 1.21 [Cl, 1.16-to-1.26]; all-cause mortality: cTnT HR per 1-SD 1.31 [Cl, 1.13-to-1.53]; and $\mathrm{CTnI} H R$ per 1-SD $1.14[\mathrm{Cl}, 1.06$-to-1.22]). These differences were significant $(p<.01)$ in metaregression analyses for cardiovascular mortality but did not reach statistical significance for all-cause mortality.

\section{Conclusions}

Elevated, basal cTnT and cTnl show robust associations with increased risk of cardiovascular and allcause mortality during follow-up in the general population. 


\section{Introduction}

Cardiac troponins are the preferred biomarkers in the diagnostic work up for non-ST elevation myocardial infarction (NSTEMI) ${ }^{1,2}$. Troponin assays target either cardiac troponin T or cardiac troponin I, both sarcomere components of the heart ${ }^{3}$. The release of cardiac troponin in the peripheral blood is strongly associated with myocardial injury ${ }^{4}$. The recent introduction of high-sensitive cardiac troponin assays has expedited the early diagnosis of NSTEMI, but also resulted in the detection of previously unnoticed cardiac troponin levels in various patient groups without acute cardiac injury such as chronic kidney disease, chronic heart failure, and even in apparently healthy subjects ${ }^{5,6}$. Subsequently, several studies have shown that even these minimal increases of cardiac troponin $\mathrm{T}$ and I are associated with unfavorable outcomes in various patient groups ${ }^{6-10}$.

The current guidelines recommend either cardiac troponin $T$ and I for de diagnosis of acute myocardial infarction, suggesting equivalent diagnostic performance of troponin $T$ and I in the acute situation ${ }^{11}$. In contrast, cardiac troponin $\mathrm{T}$ seems to have greater prognostic accuracy than cardiac troponin I in the acute setting ${ }^{12}$. Potential differences in prognostic value between basal cardiac troponin $T$ and I concentrations in subjects from the general population are largely unexplored. In addition, the quantitative relationship between elevated cardiac troponin levels, also below the $99^{\text {th }}$ percentile, and the magnitude of risk for adverse events has not been systematically assessed.

To address these knowledge gaps, we conducted a systematic review and meta-analysis of the available evidence from published prospective cohort studies. Specifically, we quantified the relationship between basal levels of high-sensitive cardiac troponins and cardiovascular and all-cause mortality during follow-up in the general population. In addition, the prognostic performance of high-sensitive cardiac troponin $T$ and I assays was separately assessed and compared. 


\section{Methods}

\section{Data sources and searches}

Medline, Embase and the Cochrane Library were searched from inception through October 2016. Also the reference lists of all relevant articles and Web of Science (for prospective citations of key publications) were checked for any additional articles. The PubMed search terms were (mortality OR death OR 'Mortality'[Mesh]) AND (troponin OR 'Troponin'[Mesh]) AND (predictive OR prediction OR prognostic OR prognosis OR 'Prognosis'[Mesh] risk OR 'Risk'[Mesh]) AND (follow-up OR prospective OR cohort). We adapted this search strategy for searches of Embase and the Cochrane Library. The search was restricted to English, Dutch, French and German documents.

The protocol for this study was published on the International Prospective Register of Systematic Reviews, or PROSPERO. The registration number was CRD42014006964.

\section{Study selection}

Study selection was performed by two independent investigators (N.v.d.L. and L.K.). We included all prospective cohort studies that evaluated the prognostic value of basal highsensitive cardiac troponin T and I levels in subjects from the general population (without any suspected acute event or surgery at the time of sampling) for cardiovascular and all-cause mortality during follow-up.

Studies were excluded from the meta-analysis when a) the duration of follow-up was less than one year, b) hazard ratios for cardiovascular or all-cause mortality were not provided (either as hazard ratio per stratum, or as hazard ratio (HR) per 1-standard deviation (SD) increase in logarithmic transformed cardiac troponin concentrations), or c) when hazard ratios were not adjusted for conventional cardiovascular risk factors (at least for age, sex, smoking, hypertension (or systolic blood pressure), diabetes mellitus (or glucose levels) and dyslipidemia (or levels of total and HDL cholesterol). When duplicate publications of data were encountered, only results from the most recent publication were considered. Discrepancies between reviewers were resolved in the presence of a third reviewer (S.M.). 


\section{Data Extraction and Quality Assessment}

We extracted data using a customized and validated extraction form. Data on study characteristics (authors, publication year, journal, study design, sample size, country and duration of follow-up), participants' characteristics (age, sex, history of CVD, hypertension, dyslipidemia, diabetes), cardiovascular and all-cause mortality rate, cardiac troponin assay, hazard ratios for cardiovascular and all-cause mortality, the factors for which the hazard ratios had been adjusted for, and C-statistics and reclassification were retrieved. When multiple cardiac troponin assays of the same kind (cardiac troponin I or T) were presented, we used the data from the assay that was commercially available and most commonly used in clinical practice for our analyses. Two authors (N.v.d.L. and L.K.) performed the dataextraction separately. Discrepancies between reviewers were resolved in presence of a third reviewer (S.M.).

To assess study quality, each study was evaluated for selection bias, study design, confounders, blinding, data collection methods, and withdrawals/dropouts according to the Effective Public Health Practice Project (EPHPP) 'Quality Assessment Tool For Quantitative Studies'13. Two authors (N.v.d.L. and L.K.) performed quality assessment separately, disagreements were resolved by consensus in the presence of a third review author (S.M.)

\section{Data Synthesis and Analysis}

To quantitatively assess the relationship between basal cardiac troponin levels and cardiovascular and all-cause mortality during follow-up, we used hazard ratios HR per 1-SD increase in logarithmic transformed cardiac troponin levels. When hazard ratios were provided for stratified cardiac troponin concentrations only, we mathematically derived HR per 1-SD increase in logarithmic transformed cardiac troponin concentration. This estimation was based on the assumption that logarithmic transformed cardiac troponin levels are normally distributed in the population ${ }^{14-16}$, and consisted of the following three steps: 1) we extracted the cumulative distribution of the study population and calculated the associated z-scores based on the cumulative standard normal distribution, 2) we calculated the mean and standard deviation based on the z-scores $(z=(x-\mu) / \sigma$, where $x$ is the log-transformed 
cardiac troponin concentration acting as upper limit of the stratum (continuity correction was performed), $\mu$ is the mean, and $\sigma$ is the standard deviation), and 3) we constructed a biomarker concentration-risk curve (x-axis: log-transformed medians for the strata, $y$-axis: associated hazard ratios) for which we calculated the slope (hazard ratio/log cardiac troponin) and transformed this into HR per 1-SD increase in logarithmic transformed cardiac troponin level. This method was validated in two studies that reported hazard ratios for both stratified data and log-transformed cardiac troponin as a continuous variable ${ }^{17,18}$. We found a minimal differences between the reported and the calculated HR per 1-SD in these two studies (2-8\% difference).

For the meta-analysis, we used full-model adjusted hazard ratios (at least adjusted for the conventional cardiovascular risk factors age, sex, smoking, hypertension (or systolic blood pressure), diabetes mellitus (or glucose levels), and dyslipidemia (or levels of total and HDL cholesterol). As a result of this approach the number of adjusting factors in the full-model differs between studies. Nevertheless, this method ensures that we correct for all significant confounders in the individual studies. Since cardiac troponins are strongly associated with cardiovascular disease, the set of adjusting factors mainly consists of cardiovascular risk factors. The adjusted HRs per 1-SD were pooled using a random-effects model and summarized using forest plots.

Examination of the impact of potential moderator variables on the study effect size was done by meta-regression analyses. Potential moderator variables included 1) type of cardiac troponin assay, 2) calculated versus reported HRs, 3) study quality, 4) geographic region, and 5) mean age of the population. A sensitivity analysis was applied to examine the effect of the inclusion of studies, that did not adjust for the minimal set of six conventional risk factors, on the robustness of the pooled results.

The MOOSE guidelines ${ }^{19}$ for meta-analysis of observational studies were followed and PRISMA criteria were performed for the search methodology (Figure 1). Publication bias was evaluated using Egger regression. Heterogeneity between studies was estimated using $\left.\right|^{2} 20$. For all analyses, a 2-tailed p-value less than .05 was considered statistically significant. All statistical analyses were performed with the Stata software package (Stata/IC version 13.1). 


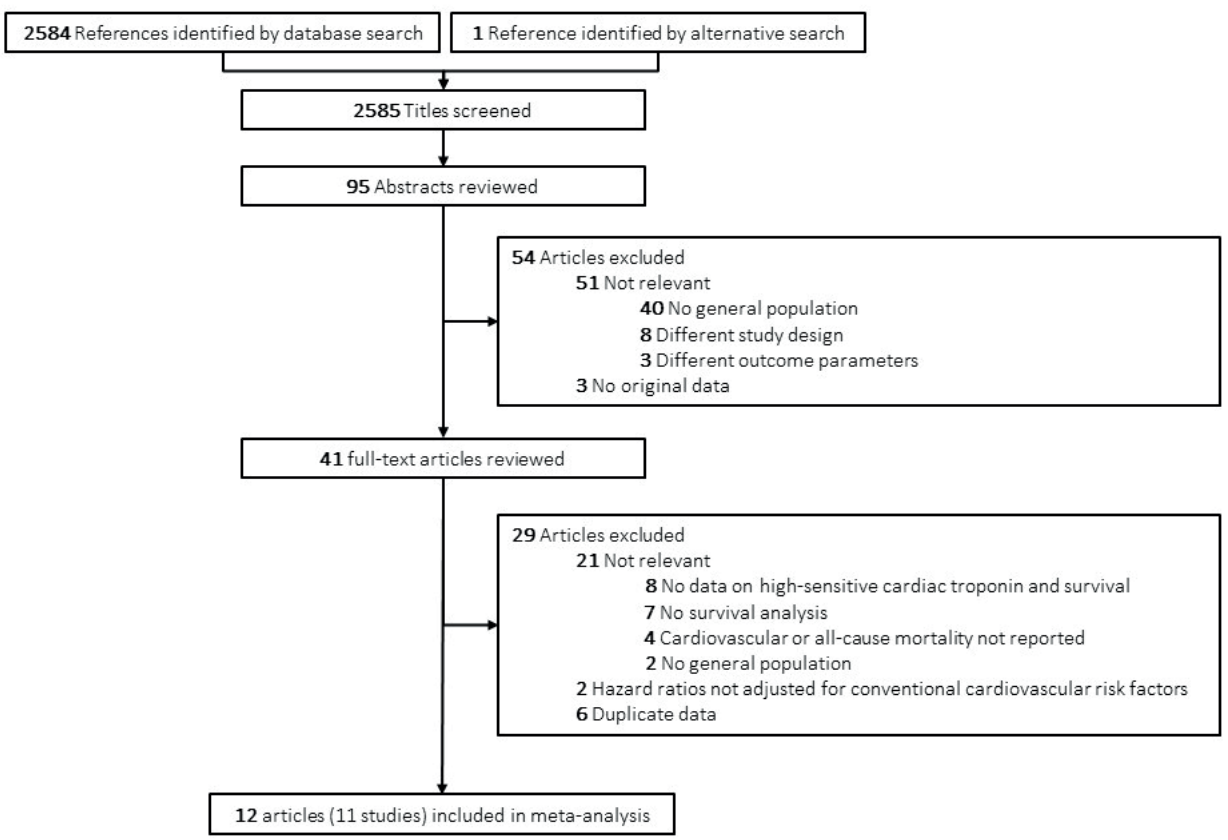

Figure 1. Flow chart of the study selection.

\section{Results}

\section{Study characteristics}

The literature search protocol is depicted in Figure 1. Our initial search identified 2585 citations. Based on titles and abstracts, 41 articles were considered potentially eligible. After full text evaluation 29 articles were excluded: eight articles did not report hazard ratio's for high-sensitive cardiac troponin concentrations and survival, seven did not perform a survival analysis, four did not report on cardiovascular or all-cause mortality as outcome measures, two were not performed in the general population, and six articles reported data that were also published in more recent articles. In two studies the reported hazard ratios were not adjusted for the minimal list of conventional risk factors. Since the adjustment for these factors is one of the inclusion criteria, those studies were excluded. Hence, a total of 11 studies (12 articles) (65019 participants) were eventually included in the meta-analysis; seven studies (45956 participants) for cardiovascular mortality ${ }^{15,17,21-25}$ and ten studies 
(48679 participants) for all-cause mortality $15,17,18,22-24,26-29$. Five studies reported hazard ratios related to cardiac troponin $\mathrm{T}$ levels ${ }^{17,18,21-23,26}$, another five studies reported on cardiac troponin I levels ${ }^{15,24,25,27-29}$. Clinical and demographic characteristics are shown in Table 1. The median follow-up duration of all studies was between 3.8 and 20 years. The percentage of individuals with detectable cardiac troponin levels varied from 27 to $96 \%$.

The study quality varied across studies. According to the EPHPP quality assessment too ${ }^{13}$ one study was of high methodological quality ${ }^{27}$, eight studies were of moderate quality ${ }^{17,18,21-}$ $25,28,29$ and two studies were of low methodological quality ${ }^{15,26}$. The global rating 'weak' for study quality was due to lack of information about subject inclusion, study withdrawals, and dropouts. To ascertain vital status studies used death registries $(n=5)^{17,22,24,26,29}$, medical records $(n=3)^{18,21,27,28}$, the combination of medical records and death registries $(n=2)^{15,25}$, and the combination of interviews, medical records and a death register $(n=1)^{23}$. All included studies provided hazard ratios adjusted for at least six conventional cardiovascular risk factors, described in the methods section. Quality assessment scores and additional adjustments made by the individual studies are presented in Supplemental Table 1.

\section{Association between cardiac troponin concentration and mortality}

We pooled adjusted HRs per 1-SD for all studies using a random-effects model. Pooling of the results from the seven included studies for cardiovascular mortality showed a significant association between increased basal cardiac troponin levels and an elevated hazard ratio for cardiovascular mortality (HR per 1-SD 1.29, 95\% confidence interval Cl 1.20 to 1.38). Cardiac troponin T was significantly stronger associated with cardiovascular mortality compared to cardiac troponin I (meta-regression analysis p<.01; HR per 1-SD 1.37, 95\% confidence interval 1.23 to 1.52 and HR per 1-SD 1.21,95\% confidence interval 1.16 to 1.26, for cardiac troponin $\mathrm{T}$ and I, respectively). The forest plots of the random-effects pooled data for all-cause mortality are shown in Figure 2. 


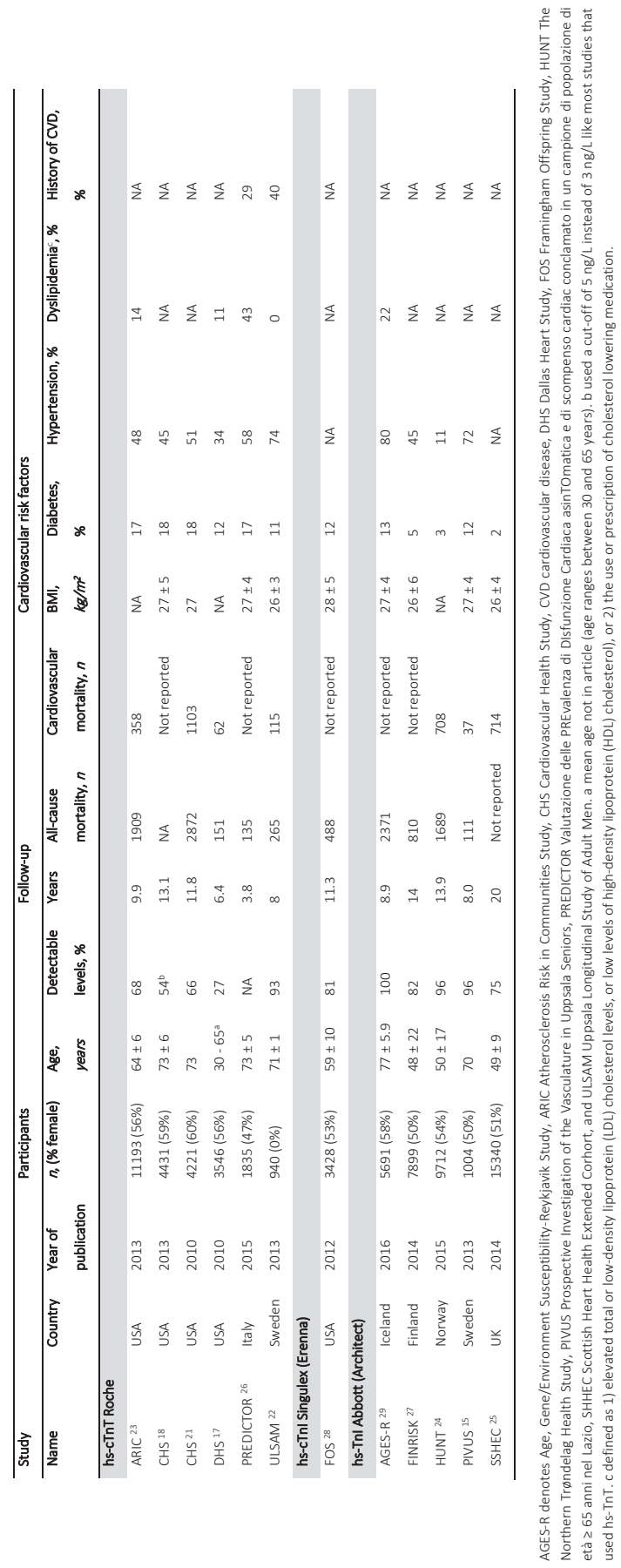


Pooling of the results from the nine included studies for all-cause mortality showed a significant association between increased basal cardiac troponin levels and an elevated hazard ratio for all-cause mortality (HR per 1-SD 1.18, 95\% confidence interval Cl 1.11 to 1.26). Considering the trend towards different effect sizes (HR per 1-SD) for cardiac troponin T and I shown by meta-regression analysis ( $p=.09)$, we performed stratified analyses for both isoforms of cardiac troponin. Basal cardiac troponin $T$ levels translated to a higher hazard ratio for all-cause mortality ((HR per 1-SD 1.31, 95\% confidence interval 1.13 to 1.53) versus $(1.14,1.06$ to 1.22$))$. The forest plots of the random-effects pooled data for all-cause mortality are shown in Figure 3.

\section{Heterogeneity and publication bias assessment}

Our meta-analysis revealed substantial heterogeneity across studies (Figure 2 and 3). We used meta-regression analyses to identify the underlying factors. Univariable metaregression analyses pointed out that estimated versus reported hazard ratio and study quality (strong/moderate versus weak global rating) were not statistically significant predictors, whereas the assay-type (high-sensitive cardiac troponin T or I) was statistically significant for cardiovascular mortality $(p<.01)$ but did not reach significance for all-cause mortality ( $p=.09)$. Besides, age was a borderline significant factor in meta-regression analysis for cardiac troponin I and all-cause mortality $(p=0.05)$, indicating that older age is associated with a stronger correlation between troponin concentration and risk.

One SD increase in basal cardiac troponin T levels was associated with a higher HR for cardiovascular and all-cause mortality during follow-up than a similar increase in troponin I. Stratified analyses for cardiac troponin $T$ and I decreased heterogeneity across troponin $T$ studies $\left(\mathrm{I}^{2}=37.9 \%, \mathrm{p}=.18\right.$ and $\mathrm{I}^{2}=62.7 \%, \mathrm{p}=.03$, for cardiovascular and all-cause mortality, respectively). Across studies with cardiac troponin I, heterogeneity for cardiovascular mortality decreased $\left(I^{2}=.0 \%, p=.40\right)$, but remained high for all-cause mortality $\left(I^{2}=83.4 \%\right.$, $\mathrm{p}<.01)$.

Egger's regression analysis was not indicative for publication bias $(p=.50$ and $p=.68$, for cardiovascular and all-cause mortality, respectively) (Supplemental Figure 1 and 2). 
A

$\begin{array}{ll}\text { study } & \text { ES }(95 \% \mathrm{Cl}) \\ & \\ \text { DHS } & 1.41(1.07,1.86) \\ \text { ARIC } & 1.71(1.32,2.21) \\ \text { PIVUS } & 1.31(1.10,1.55) \\ \text { ULSAM } & 1.20(1.00,1.45) \\ \text { CHS } & 1.36(1.29,1.43) \\ \text { SHHEC } & 1.18(1.12,1.25) \\ \text { HUNT } & 1.23(1.15,1.31)\end{array}$

$\%$ Weight

Overall

$1^{2}=70.4 \%, p=0.002$

$1.29(1.20,1.38)$

$B$

study

ES $(95 \%$ CI)

DHS $\quad 1.41(1.07,1.86)$

ARIC

$1.71(1.32,2.21)$

ULSAM

$1.20(1.00,1.45)$

CHS

$1.36(1.29,1.43)$

Cardiac troponin T $1.37(1.23,1.52)$

$r^{2}=37.9 \%, p=0.184$

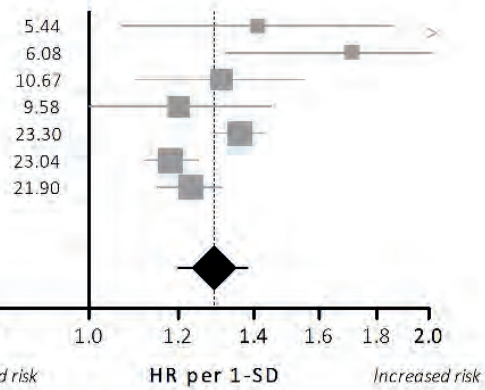

Decreased risk

HR per 1-SD

$\%$ Weight

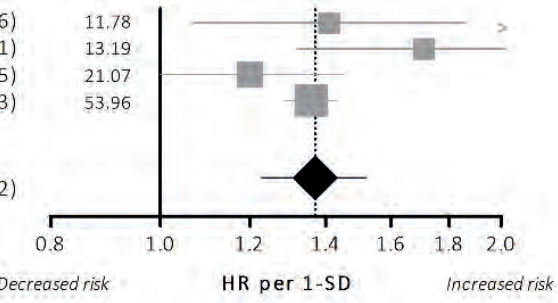

C

Decreased risk

HR per 1 -SD

$\%$ Weight

study

ES $(95 \%$ Cl)

PIVUS

$1.31(1.10,1.55)$

SHHEC

$1.18(1.12,1.25)$

HUNT

$1.23(1.15,1.31)$

Cardiac troponin I $1.21(1.16,1.26)$

$P^{2}=0.0 \%, p=0.403$

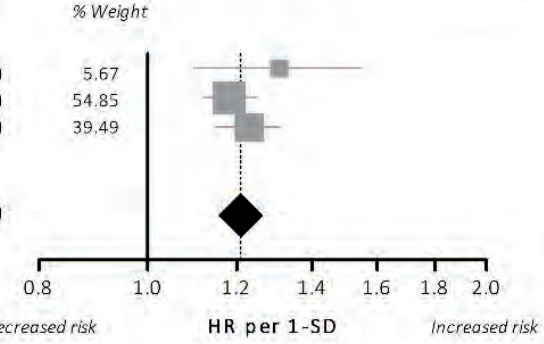

Figure 2. Association between one standard deviation increase in basal cardiac troponin concentrations and hazard ratio for cardiovascular mortality in the general population. The boxes and lines in the forest plots indicate the hazard ratio for cardiovascular mortality per standard deviation increase (HR per 1-SD) in cardiac troponin concentrations for individual studies and a pooled estimate (random-effects model) for all studies (panel A), studies on cardiac troponin T (panel B), and studies on cardiac troponin I (panel C). 
A

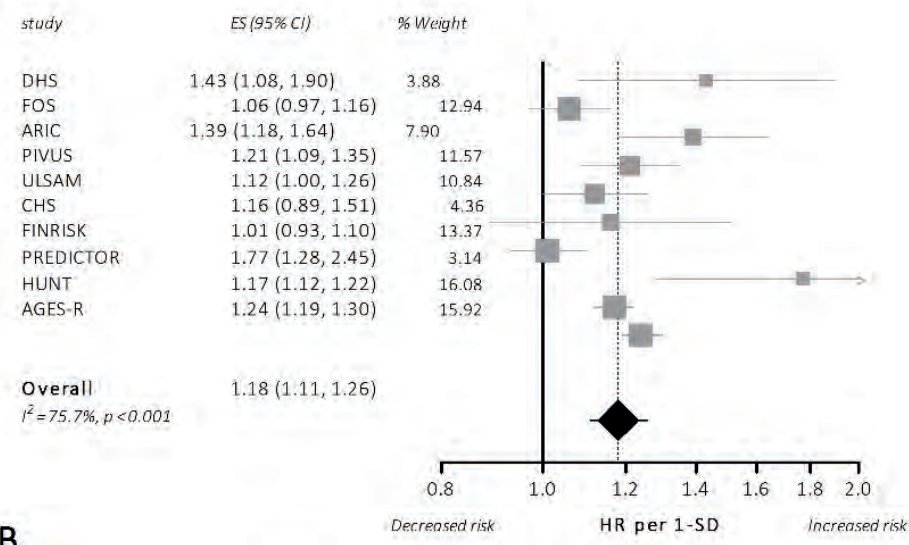

B

study

ES $(95 \%$ CI)

\% Weight

DHS

ARIC

ULSAM

CHS

$1.43(1.08,1.90)$

$1.39(1.18,1.64)$

$1.12(1.00,1.26)$

$1.16(0.89,1.51)$

PREDICTOR

$1.77(1.28,2.45)$

Cardiac troponin T $1.31(1.13,1.53)$

$t^{2}=62.7 \%, p=0.030$

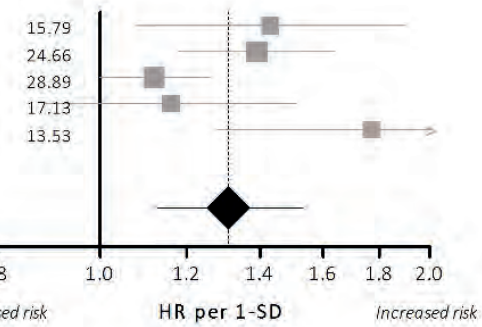

C

Decreased risk

HR per 1-SD

$\%$ Weight

study

ES $(95 \%$ C $)$

FOS

PIVUS

$1.06(0.971 .16)$

$1.21(1.09,1.35)$

FINRISK

$1.01(0.93,1.10)$

HUNT

$1.17(1.12,1.22)$

AGES-R

$1.24(1.19,1.30)$

Cardiac troponin I $1.14(1.06,1.22)$

$r^{2}=83.4 \%, p<0.001$

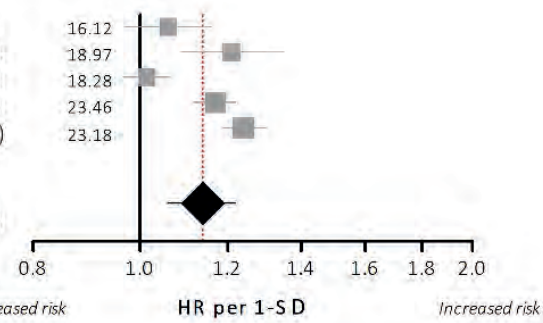

Figure 3. Association between one standard deviation increase in basal cardiac troponin concentrations and hazard ratio for all-cause mortality in the general population. The boxes and lines in the forest plots indicate the hazard ratio for all-cause mortality per standard deviation increase (HR per 1-SD) in cardiac troponin concentrations for individual studies and a pooled estimate (random-effects model) for all studies (panel A), studies on cardiac troponin $\mathrm{T}$ (panel B), and studies on cardiac troponin I (panel C). 


\section{Sensitivity analysis}

For all-cause mortality two studies were excluded because they did not correct for at least the six conventional cardiovascular risk factors. To verify that pooled hazard ratios were robust and independent, a sensitivity analysis including those two studies was performed. Random-effects pooling after the addition of two additional studies ${ }^{30,31}$ revealed similar results regarding all-cause mortality and reduced heterogeneity for cardiac troponin T (HR per 1-SD 1.30, 95\% confidence interval 1.16 to $\left.1.47 ; \mathrm{I}^{2}=52.9 \%(\mathrm{p}=.060)\right)$, cardiac troponin I $\left(1.14,1.07\right.$ to $\left.1.22 ; I^{2}=79.3 \%(p<.01)\right)$ and the combined analysis $\left(1.19,1.12\right.$ to $1.26 ; I^{2}=$ $71.3 \%(p<.01))$. The meta-regression analysis that was performed after the inclusion of two additional studies ${ }^{30,31}$ showed a significant effect of assay-type (cardiac troponin T versus I) on the observed heterogeneity $(p=.05)$.

\section{Translation to clinical practice}

Together, the pooled data show that elevated basal cardiac troponin levels in the general population are associated with increased mortality during follow-up, and suggest that a slightly stronger association for cardiac troponin T than for cardiac troponin I. To relate the pooled summary measures per standard deviation, to a clinically comprehensible risk estimation tool, we transformed HR per 1-SD to absolute cardiac troponin concentrations and corresponding hazard ratios for all high-sensitive troponin assays that were included in the meta-analysis. Figure 4 depicts the relation between basal cardiac troponin $\mathrm{T}$ and I concentrations and mortality during follow-up.

The majority of included studies performed discrimination and reclassification statistics (Supplemental Table 2) 15,17,21,22,24,25,27,29. The results from these analyses suggest small, but significant improvements in (established) prediction models based on established risk factors after the inclusion of high-sensitive cardiac troponin $\mathrm{T}$ or I. 
A

Cardiac troponin T-cardiovascular mortality

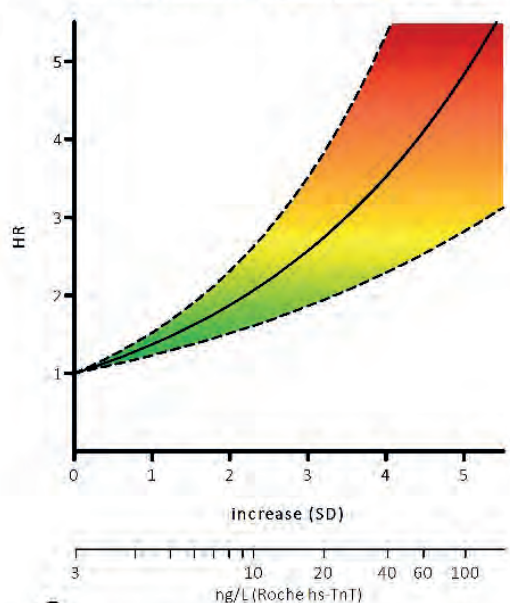

C

Cardiac troponin T-all-cause mortality

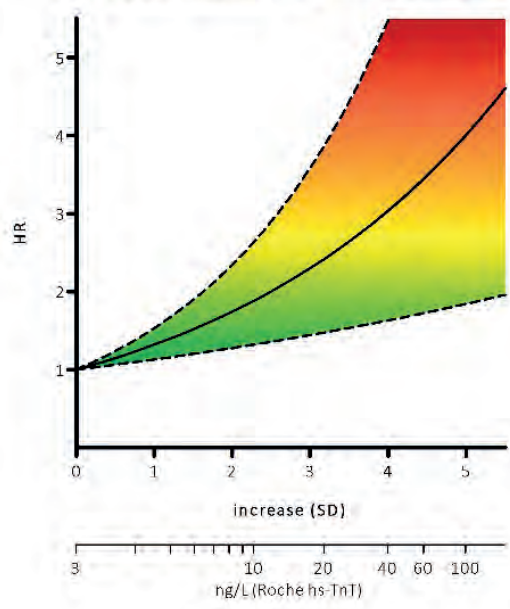

B

Cardiac troponin I-cardiovascular mortality

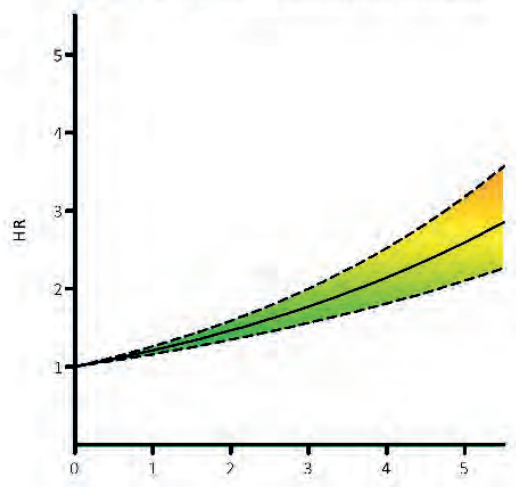

increase (SD)

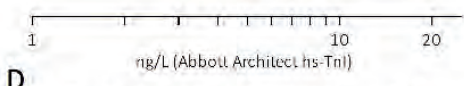

Cardiac troponin I-all-cause mortality

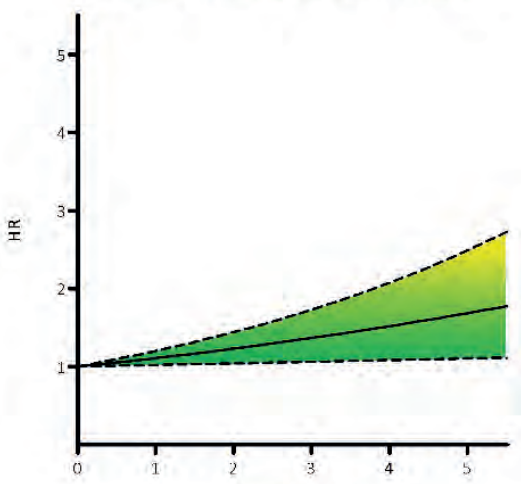

increase (SD)

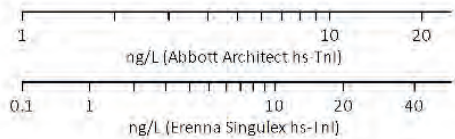

Figure 4. Cardiac troponin levels and corresponding hazard ratios. HR per 1-SD increase was transformed to absolute cardiac troponin concentrations and corresponding hazard ratios for all high-sensitive troponin assays that were included in the meta-analysis. 


\section{Discussion}

This meta-analysis with data on 65019 participants from 11 prospective cohorts shows that basal elevated cardiac troponin concentrations, also below the $99^{\text {th }}$ percentile, in the general population are associated with an increased risk for cardiovascular and all-cause mortality during follow-up. The pooled data analyses corroborates and extends the findings of previous studies $^{7-9,32,33}$. Besides, our results suggest that the quantitative relationship between cardiac troponin concentrations is stronger for cardiovascular than for all-cause mortality, and may be slightly stronger for cardiac troponin T than for cardiac troponin I.

The observed association between elevated cardiac troponin concentrations and an increased risk for cardiovascular mortality and, to a lesser extent, for all-cause mortality, are reflected in the discrimination and reclassification statistics of the included studies. These analyses indicate that the addition of cardiac troponins may cause a small, but significant improvement in risk prediction models $15,17,21,22,24,25,27,29$. Some recent studies, but not all, suggest that the addition of cardiac troponin I to existing risk prediction models may be particularly useful among a subpopulation, especially in older and female subjects $24,25,29,33$. Before cardiac troponins can be actually implemented in clinical practice, it is necessary to identify the suitable target population and the best combination of biomarkers for risk prediction, also from a cost-effectiveness point of view.

The observed differences in the quantitative relationships of high-sensitive cardiac troponin $T$ and I are clinically and scholarly interesting and contrast with demonstrated equivalent performance of both assays in the acute setting, i.e. for diagnosing acute myocardial infarction $^{34}$. We can only speculate about the factors that might underlie these apparent prognostic differences. A first factor concerns the analytical aspects of the distinct cardiac troponin assays. It is conceivable that intrinsic assay differences, including the use of different antibodies ${ }^{35}$, may affect distinctive power of troponin assays with direct consequences for prognostic performance. A second factor involves the release of cardiac troponins. The mechanism of chronic cardiac troponin release is not elucidated, but recent observations suggest differences in release patterns of cardiac troponin T and $\mathrm{I}^{36}$. Third, post- 
release modification like fragmentation, complex formation, and elimination of cardiac troponins may differ between both cardiac troponins ${ }^{37,38}$.

This meta-analysis has several strengths, including its large patient populations, standardization of the outcome measure and the comparison of cardiac troponin $\mathrm{T}$ and I. Limitations of our study merit consideration: First, despite the consistent adjustment for at least six established cardiovascular risk factors and the use of a standardized outcome measure (HR per 1-SD), heterogeneity was substantial, which persisted in assay-stratified analyses, in particular across all-cause mortality studies. Meta-regression analyses did not identify other suggestive factors that contributed to heterogeneity. A likely, remaining source of heterogeneity is the diversity in populations, caused by variable in- and exclusion criteria of the individual studies. Nonetheless, despite the detected heterogeneity, all studies revealed the same direction of effect, with increased mortality upon higher levels of circulating cardiac troponin. Second, due to the fact that we did not have access to the individual, data and that the percentage of included women showed minimal variation, we were unable to examine the effect of gender. Therefore we cannot confirm that gender affects the relation between cardiac troponin concentrations and the risk on cardiovascular and all-cause mortalit $24,30,33$. With respect to age, we could only examine the effect of the mean age across the included studies. In line with previous observations ${ }^{33}$, we found a borderline significant association between increasing age and a stronger correlation between troponin I and all-cause mortality. We cannot exclude that the variation in in- and exclusion criteria and adjusting factors might have masked a more explicit effect of age. Third, HR per 1-SD from stratified data has been mathematically derived. However, validation of this method in two studies revealed minimal discrepancies, and this approach to standardize riskestimates enabled us to pool rather heterogeneously presented data, and to perform a metaanalysis with substantial statistical power and long-term follow-up. Fourth, the measurement and definition of mortality outcomes differs across studies. The definition of cardiovascular mortality varied slightly across studies, but in general it concerned the ICD-10 diagnosis I00199. 


\section{Conclusion}

Elevated, basal cardiac troponin T and I levels are significantly associated with increased risk of cardiovascular and all-cause mortality during follow-up in the general population. The results of this meta-analysis suggest this association is stronger for cardiovascular than for all-cause mortality. The observation that the quantitative relationship between cardiac troponin concentration and mortality risk may be stronger for cardiac troponin $T$ than for cardiac troponin I is an interesting finding that requires further research. In addition, future studies should focus on further establishment and validation of cardiac troponins for the prediction of long-term outcomes. 


\section{References}

1. Thygesen K, Alpert JS, Jaffe AS, et al. Third universal definition of myocardial infarction. J Am Coll Cardiol 2012;60:1581-98.

2. Al-Saleh A, Alazzoni A, Al Shalash S, et al. Performance of the high-sensitivity troponin assay in diagnosing acute myocardial infarction: systematic review and meta-analysis. CMAJ open 2014;2:E199-207.

3. Katrukha IA. Human cardiac troponin complex. Structure and functions. Biochemistry (Mosc) 2013;78:1447-65.

4. de Lemos JA. Increasingly sensitive assays for cardiac troponins: a review. Jama 2013;309:2262-9.

5. Giannitsis E, Katus HA. Cardiac troponin level elevations not related to acute coronary syndromes. Nat Rev Cardiol 2013;10:623-34.

6. Collinson P. The role of cardiac biomarkers in cardiovascular disease risk assessment. Current opinion in cardiology 2014;29:366-71.

7. Ahmed AN, Blonde K, Hackam D, lansavichene A, Mrkobrada M. Prognostic significance of elevated troponin in non-cardiac hospitalized patients: a systematic review and meta-analysis. Annals of medicine 2014;46:653-63.

8. Michos ED, Wilson LM, Yeh HC, et al. Prognostic value of cardiac troponin in patients with chronic kidney disease without suspected acute coronary syndrome: a systematic review and meta-analysis. Annals of internal medicine 2014;161:491-501.

9. Khan NA, Hemmelgarn BR, Tonelli M, Thompson CR, Levin A. Prognostic value of troponin $T$ and I among asymptomatic patients with end-stage renal disease: a meta-analysis. Circulation 2005;112:3088-96.

10. Lipinski MJ, Baker NC, Escarcega RO, et al. Comparison of conventional and high-sensitivity troponin in patients with chest pain: a collaborative meta-analysis. Am Heart J 2015;169:6-16 e6.

11. Roffi M, Patrono C, Collet JP, et al. 2015 ESC Guidelines for the management of acute coronary syndromes in patients presenting without persistent ST-segment elevation: Task Force for the Management of Acute Coronary Syndromes in Patients Presenting without Persistent ST-Segment Elevation of the European Society of Cardiology (ESC). Eur Heart J 2016;37:267-315.

12. Haaf $P$, Reichlin $T$, Twerenbold $R$, et al. Risk stratification in patients with acute chest pain using three highsensitivity cardiac troponin assays. European heart journal 2014;35:365-75.

13. Thomas BH, Ciliska D, Dobbins M, Micucci S. A process for systematically reviewing the literature: providing the research evidence for public health nursing interventions. Worldviews on evidence-based nursing / Sigma Theta Tau International, Honor Society of Nursing 2004;1:176-84.

14. Koerbin G, Potter JM, Abhayaratna WP, Telford RD, Hickman PE. The distribution of cardiac troponin I in a population of healthy children: lessons for adults. Clin Chim Acta 2013;417:54-6.

15. Eggers KM, Venge P, Lindahl B, Lind L. Cardiac troponin I levels measured with a high-sensitive assay increase over time and are strong predictors of mortality in an elderly population. Journal of the American College of Cardiology 2013;61:1906-13.

16. Venge P, Johnston N, Lindahl B, James S. Normal plasma levels of cardiac troponin I measured by the highsensitivity cardiac troponin I access prototype assay and the impact on the diagnosis of myocardial ischemia. Journal of the American College of Cardiology 2009;54:1165-72.

17. de Lemos JA, Drazner MH, Omland T, et al. Association of troponin T detected with a highly sensitive assay and cardiac structure and mortality risk in the general population. Jama 2010;304:2503-12.

18. Hussein AA, Gottdiener JS, Bartz TM, et al. Cardiomyocyte injury assessed by a highly sensitive troponin assay and sudden cardiac death in the community: the Cardiovascular Health Study. Journal of the American College of Cardiology 2013;62:2112-20.

19. Stroup DF, Berlin JA, Morton SC, et al. Meta-analysis of observational studies in epidemiology: a proposal for reporting. Meta-analysis Of Observational Studies in Epidemiology (MOOSE) group. Jama 2000;283:2008-12.

20. Higgins JP, Thompson SG. Quantifying heterogeneity in a meta-analysis. Statistics in medicine 2002;21:1539-58. 21. deFilippi $\mathrm{CR}$, de Lemos JA, Christenson RH, et al. Association of serial measures of cardiac troponin $\mathrm{T}$ using a sensitive assay with incident heart failure and cardiovascular mortality in older adults. Jama 2010;304:2494-502. 
22. Eggers KM, Al-Shakarchi J, Berglund L, et al. High-sensitive cardiac troponin T and its relations to cardiovascular risk factors, morbidity, and mortality in elderly men. American heart journal 2013;166:541-8.

23. Oluleye OW, Folsom AR, Nambi V, Lutsey PL, Ballantyne CM, Investigators AS. Troponin T, B-type natriuretic peptide, C-reactive protein, and cause-specific mortality. Annals of epidemiology 2013;23:66-73.

24. Omland T, de Lemos JA, Holmen OL, et al. Impact of sex on the prognostic value of high-sensitivity cardiac troponin I in the general population: the HUNT study. Clinical chemistry 2015;61:646-56.

25. Zeller T, Tunstall-Pedoe H, Saarela O, et al. High population prevalence of cardiac troponin I measured by a highsensitivity assay and cardiovascular risk estimation: the MORGAM Biomarker Project Scottish Cohort. European heart journal 2014;35:271-81.

26. Masson S, Agabiti N, Vago T, et al. The fibroblast growth factor-23 and Vitamin D emerge as nontraditional risk factors and may affect cardiovascular risk. Journal of internal medicine 2015;277:318-30.

27. Neumann JT, Havulinna AS, Zeller T, et al. Comparison of three troponins as predictors of future cardiovascular events--prospective results from the FINRISK and BiomaCaRE studies. PloS one 2014;9:e90063.

28. Wang TJ, Wollert KC, Larson MG, et al. Prognostic utility of novel biomarkers of cardiovascular stress: the Framingham Heart Study. Circulation 2012;126:1596-604.

29. Thorsteinsdottir I, Aspelund T, Gudmundsson E, et al. High-Sensitivity Cardiac Troponin I Is a Strong Predictor of Cardiovascular Events and Mortality in the AGES-Reykjavik Community-Based Cohort of Older Individuals. Clinical chemistry 2016;62:623-30.

30. Dallmeier D, Denkinger M, Peter $R$, et al. Sex-specific associations of established and emerging cardiac biomarkers with all-cause mortality in older adults: the ActiFE study. Clinical chemistry 2015;61:389-99.

31. McKie PM, AbouEzzeddine OF, Scott CG, et al. High-sensitivity troponin I and amino-terminal pro--B-type natriuretic peptide predict heart failure and mortality in the general population. Clin Chem 2014;60:1225-33.

32. Sze J, Mooney J, Barzi F, Hillis GS, Chow CK. Cardiac Troponin and its Relationship to Cardiovascular Outcomes in Community Populations - A Systematic Review and Meta-analysis. Heart Lung Circ 2015.

33. Blankenberg S, Salomaa V, Makarova N, et al. Troponin I and cardiovascular risk prediction in the general population: the BiomarCaRE consortium. European heart journal 2016;37:2428-37.

34. Irfan A, Reichlin T, Twerenbold R, et al. Early diagnosis of myocardial infarction using absolute and relative changes in cardiac troponin concentrations. The American journal of medicine 2013;126:781-8 e2.

35. Apple FS, Collinson PO, Biomarkers ITFoCAoC. Analytical characteristics of high-sensitivity cardiac troponin assays. Clinical chemistry 2012;58:54-61.

36. Klinkenberg L, Wildi K, van der Linden N, et al. Diurnal Rhythm of Cardiac Troponin: Consequences for the Diagnosis of Acute Myocardial Infarction. Clinical chemistry 2016.

37. Streng AS, de Boer D, van der Velden J, van Dieijen-Visser MP, Wodzig WK. Posttranslational modifications of cardiac troponin T: an overview. J Mol Cell Cardiol 2013;63:47-56.

38. Martin AF. Turnover of cardiac troponin subunits. Kinetic evidence for a precursor pool of troponin-I. J Biol Chem 1981;256:964-8. 


\section{Supplemental information}

\section{Supplemental Figures}

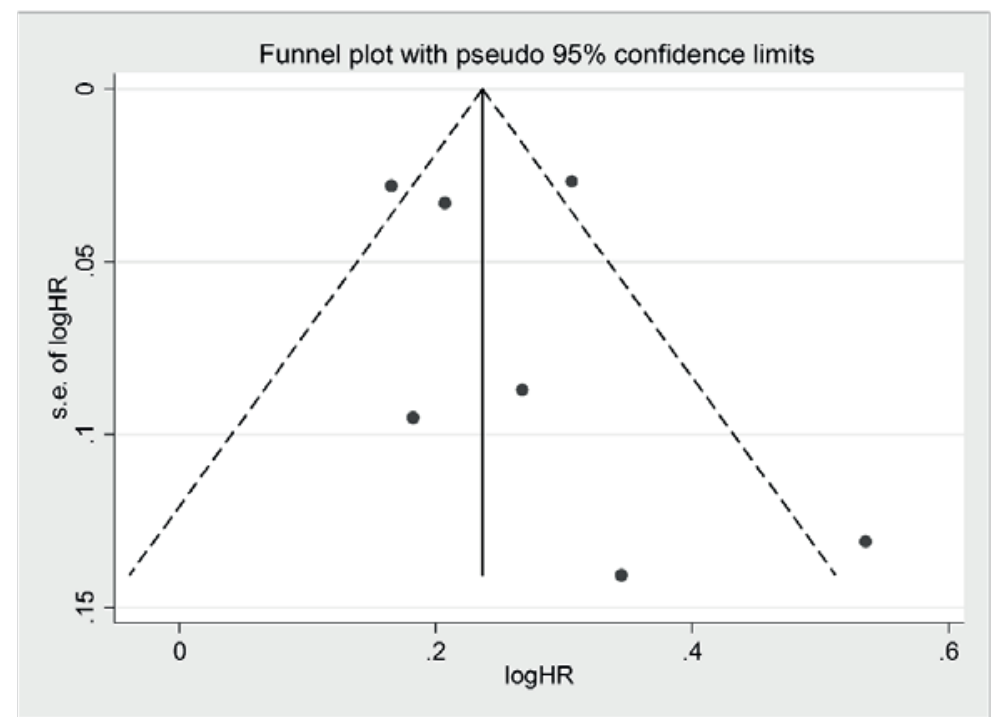

Supplemental Figure S1. Funnel plot cardiovascular mortality

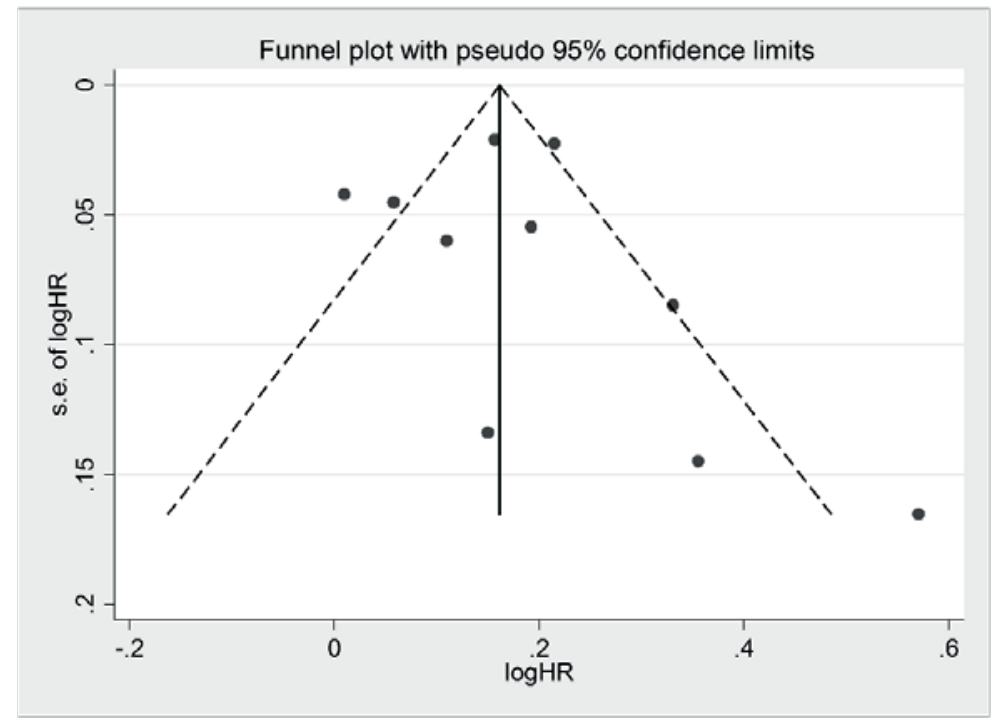

Supplemental Figure S2. Funnel plot all-cause mortality 


\section{Supplemental Tables}

Supplemental table 1a. Quality assessment (EPHPP quality assessment tool)

\begin{tabular}{|c|c|c|c|c|c|c|c|c|c|}
\hline & $\begin{array}{l}\frac{\mathscr{N}}{0} \\
\frac{0}{0} \\
\frac{0}{0} \\
\frac{U}{U} \\
\frac{\mathscr{U}}{\mathscr{N}}\end{array}$ & 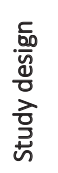 & 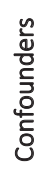 & 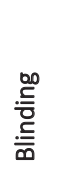 & 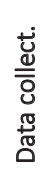 & 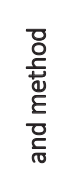 & 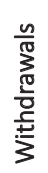 & $\begin{array}{l}\text { ํㅗ } \\
\text { 음 } \\
\text { 은 } \\
\text { 음 }\end{array}$ & 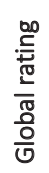 \\
\hline Oluleye, Ann Epidemiol 2013 & $+/-$ & $+/-$ & + & $+/-$ & & + & ${ }^{2}$ & - & $+/-$ \\
\hline Hussein, J Am Coll Cardiol 2013 & $+/-$ & $+/-$ & + & $+/-$ & & + & & - & $+/-$ \\
\hline deFilippi, JAMA 2010 & $+/-$ & $+/-$ & + & $+/-$ & & + & & - & $+/-$ \\
\hline De Lemos, JAMA 2010 & $+/-$ & $+/-$ & + & $+/-$ & & + & & - & $+/-$ \\
\hline Masson, J Intern Med 2014 & - & $+/-$ & + & $+/-$ & & + & & - & - \\
\hline Eggers, Am Heart J 2013 & + & $+/-$ & + & $+/-$ & & + & & - & $+/-$ \\
\hline Neumann, PLoS One 2014 & $+/-$ & $+/-$ & + & $+/-$ & & + & r & + & + \\
\hline Wang, Circulation 2012 & $+/-$ & $+/-$ & + & $+/-$ & & + & & - & $+/-$ \\
\hline Omland, Clin Chem 2015 & $+/-$ & $+/-$ & + & $+/-$ & & + & & - & $+/-$ \\
\hline Eggers, J Am Coll Cardiol 2013 & - & $+/-$ & + & $+/-$ & & + & & - & - \\
\hline Thorsteinsdottir, Clin Chem 2016 & $+/-$ & $+/-$ & + & $+/-$ & & + & & - & $+/-$ \\
\hline Zeller, Eur Heart J 2014 & $+/-$ & $+/-$ & + & $+/-$ & & + & & - & $+/-$ \\
\hline
\end{tabular}

+ indicates strong, +/- moderate and - weak. 


\section{Supplemental Table 1b. Variables in cox regression model.}

\begin{tabular}{|c|c|}
\hline Article & Variables in multivariate cox regression analysis \\
\hline Oluleye, Ann Epidemiol 2013 & $\begin{array}{l}\text { Age, gender, race, BMI, total cholesterol, } \mathrm{HDL} \text { cholesterol, SBP, diet, sport } \\
\text { index, FEV } V_{1} \text { eGFR, smoking, drinking, hormone use, antihypertensive } \\
\text { medication, DM, history of cancer, CVD, stroke, heart failure, and respiratory } \\
\text { disease, hs-CRP and NT-proBNP }\end{array}$ \\
\hline Hussein, J Am Coll Cardiol 2013 & $\begin{array}{l}\text { Age, race, gender, smoking, SBP, serum glucose, total cholesterol, HDL } \\
\text { cholesterol, eGFR, physical activity, history of heart failure, coronary disease, } \\
\text { history of myocardial infarction, stroke, transient ischemic attack, ventricular } \\
\text { conduction delay, Q and QS abnormalities, prolonged QT interval, LVEF, LV } \\
\text { mass, CRP, NT-proBNP, use of aspirin, antihypertensives, antiarrhythmics, } \\
\text { and digoxin }\end{array}$ \\
\hline deFilippi, JAMA 2010 & $\begin{array}{l}\text { Age, gender, race, SBP, DBP, use of antihypertensives, diabetes, CHD, } \\
\text { smoking, HDL cholesterol, LDL cholesterol, NT-proBNP and CRP }\end{array}$ \\
\hline De Lemos, JAMA 2010 & $\begin{array}{l}\text { Age, gender, race/ethnicity, DM, hypertension, hypercholesterolemia, HDL } \\
\text { cholesterol, smoking, hs-CRP, CKD categories, and NT-proBNP }\end{array}$ \\
\hline Masson, J Intern Med 2014 & $\begin{array}{l}\text { Age, gender, dyslipidemia, DM, SBP, smoking, blood sample taken in the } \\
\text { winter season, eGFR, COPD, alcohol consumption, atrial fibrillation, heart } \\
\text { failure, history of ischemic heart disease, LVEF, LV mass/BSA, NT-proBNP and } \\
\text { vitamin D }\end{array}$ \\
\hline Eggers, Am Heart J 2013 & $\begin{array}{l}\text { Age, smoking, BMI, SBP, antihypertensive treatment, total cholesterol, HDL } \\
\text { cholesterol, lipid-lowering treatment, DM, and previous or prevalent cancer, } \\
\text { NT-proBNP, CRP, cystatin C }\end{array}$ \\
\hline Neumann, PLoS One 2014 & $\begin{array}{l}\text { Age, gender, total cholesterol, HDL cholesterol, SBP, hypertension } \\
\text { medication, DM, smoking, region of Finland, CRP, and NT-proBNP }\end{array}$ \\
\hline Wang, Circulation 2012 & $\begin{array}{l}\text { Age, gender, BMI, SBP, hypertension therapy, DM, smoking, total } \\
\text { cholesterol, HDL cholesterol, atrial fibrillation, major cardiovascular disease, } \\
\text { ECG left ventricular disease, heart murmur, sST2, GDF-15, BNP and hsCRP }\end{array}$ \\
\hline Omland, Clin Chem 2015 & $\begin{array}{l}\text { Age, gender, total and HDL cholesterol, SBP, treatment of hypertension, } \\
\text { smoking, diabetes, eGFR, history of myocardial infarction, CRP }\end{array}$ \\
\hline Eggers, J Am Coll Cardiol 2013 & $\begin{array}{l}\text { Gender, hypertension, DM, HDL cholesterol, LDL cholesterol, smoking, BMI, } \\
\text { eGFR, CRP, NT-proBNP and previous cardiovascular disease }\end{array}$ \\
\hline Thorsteinsdottir, Clin Chem 2016 & $\begin{array}{l}\text { Age, gender, diabetes, BMI, hypertension medication, systolic blood } \\
\text { pressure, smoking, log CRP, log creatinine, cholesterol, HDL cholesterol, log } \\
\text { triglycerides, statin medication }\end{array}$ \\
\hline Zeller, Eur Heart J 2014 & $\begin{array}{l}\text { Age, sex, cohort, family history of } \mathrm{CHD} / \text { stroke, diabetes, rheumatoid arthritis, } \\
\text { use of antihypertensives, smoking, BMI, SBP, total cholesterol, HDL } \\
\text { cholesterol }\end{array}$ \\
\hline
\end{tabular}


Supplemental Table 2. C-statistic and reclassification for the addition of hs-cTn*

All-cause mortality

\begin{tabular}{|c|c|c|c|c|c|c|c|}
\hline Study & $\begin{array}{l}\text { C-statistic } \\
\text { without hs-cTn }\end{array}$ & $\begin{array}{l}\text { C-statistic } \\
\text { with hs-cTn }\end{array}$ & p-value & NRI & p-value & IDI & $\mathrm{p}$-value \\
\hline \multicolumn{8}{|c|}{ hs-cTnT Roche } \\
\hline $\mathrm{CHS}^{21}$ & NA & NA & NA & NA & NA & NA & NA \\
\hline \multirow[t]{8}{*}{$\mathrm{DHS}^{17}$} & Model 1: & Model 1: & Model 1: & NA & NA & Model 1: & Model 1: \\
\hline & 0.793 & 0.818 & $<0.001$ & & & 0.044 & $<0.001$ \\
\hline & Model 2: & Model 2: & Model 2: & & & Model 2: & Model 2: \\
\hline & 0.793 & 0.818 & $<0.001$ & & & 0.044 & $<0.001$ \\
\hline & Model 3: & Model 3: & Model 3: & & & Model 3: & Model 3: \\
\hline & 0.806 & 0.823 & $<0.01$ & & & 0.024 & $<0.001$ \\
\hline & Model 4: & Model 4: & Model 4: & & & Model 4: & Model 4: \\
\hline & 0.821 & 0.827 & 0.02 & & & 0.010 & 0.01 \\
\hline ULSAM ${ }^{22}$ & 0.63 & 0.65 & 0.07 & NA & NA & 0.02 & $<0.001$ \\
\hline \multicolumn{8}{|c|}{ Hs-Tnl Abbott (Architect) } \\
\hline AGES-R 29 & 0.739 & 0.748 & $<0.001$ & 0.26 & NA & NA & NA \\
\hline FINRISK 27 & 0.8174 & 0.8173 & ns & -0.0040 & ns & -0.0002 & ns \\
\hline \multirow[t]{4}{*}{ HUNT ${ }^{24}$} & Women: & Women: & Women: & NA & NA & NA & NA \\
\hline & 0.934 & 0.937 & 0.011 & & & & \\
\hline & Men: & Men: & Men: & & & & \\
\hline & Not reported & Not reported & ns & & & & \\
\hline \multirow[t]{5}{*}{ PIVUS 15} & Not reported & Model 1: & Model 1: & Model 1: & Model 1: & Model 1: & Model 1: \\
\hline & & +0.003 & 0.51 & 0.231 & 0.02 & 0.014 & 0.04 \\
\hline & & Model 2: & Model 2: & Model 2: & Model 2: & Model 2: & Model 2: \\
\hline & & +0.006 & 0.60 & 0.140 & 0.17 & 0.120 & 0.07 \\
\hline & & Model 3: & Model 3: & Model 3: & Model 3: & Model 3: & Model 3: \\
\hline SSHEC $^{25}$ & NA & NA & NA & $\begin{array}{l}0.288 \\
\text { NA }\end{array}$ & NA & NA & $\begin{array}{l}0.01 \\
N A\end{array}$ \\
\hline \multicolumn{8}{|c|}{ Cardiovascular mortality } \\
\hline Study & $\begin{array}{l}\text { C-statistic } \\
\text { without hs-cTn }\end{array}$ & $\begin{array}{l}\text { C-statistic } \\
\text { with hs-cTn }\end{array}$ & p-value & NRI & $p$-value & IDI & p-value \\
\hline \multicolumn{8}{|c|}{ hs-cTnT Roche } \\
\hline $\mathrm{CHS}^{21}$ & 0.779 & 0.792 & $<0.05$ & 0.040 & $<0.05$ & 0.021 & $<0.05$ \\
\hline \multirow[t]{8}{*}{$\mathrm{DHS}^{17}$} & Model 1: & Model 1: & Model 1: & NA & NA & Model 1: & Model 1: \\
\hline & 0.832 & 0.868 & $<0.001$ & & & 0.027 & $<0.001$ \\
\hline & Model 2: & Model 2: & Model 2: & & & Model 2: & Model 2: \\
\hline & 0.833 & 0.869 & $<0.001$ & & & 0.029 & $<0.001$ \\
\hline & Model 3: & Model 3: & Model 3: & & & Model 3: & Model 3: \\
\hline & 0.865 & 0.882 & 0.02 & & & 0.010 & 0.10 \\
\hline & Model 4: & Model 4: & Model 4: & & & Model 4: & Model 4: \\
\hline & 0.889 & 0.893 & 0.37 & & & 0.005 & 0.24 \\
\hline ULSAM ${ }^{22}$ & 0.66 & 0.70 & 0.01 & NA & NA & 0.02 & 0.001 \\
\hline \multicolumn{8}{|c|}{ Hs-Tnl Abbott (Architect) } \\
\hline AGES-R 29 & NA & NA & NA & NA & NA & NA & NA \\
\hline FINRISK 27 & NA & NA & NA & NA & NA & NA & NA \\
\hline \multirow[t]{4}{*}{ HUNT ${ }^{24}$} & Women: & Women: & Women: & NA & NA & NA & NA \\
\hline & 0.875 & 0.878 & 0.003 & & & & \\
\hline & Men: & Men: & Men: & & & & \\
\hline & Not reported & Not reported & ns & & & & \\
\hline PIVUS ${ }^{15}$ & NA & NA & NA & NA & NA & NA & NA \\
\hline SSHEC 25 & 0.8362 & 0.8439 & $<0.0001$ & 0.0451 & 0.0119 & 0.0053 & 0.0150 \\
\hline
\end{tabular}


*Addition of hs-cTn to models with established risk factors:

CHS: model includes age, gender, race, SBP, DBP, use of antihypertensives, diabetes, CHD, smoking, HDL cholesterol, LDL cholesterol, NT-proBNP and CRP

DHS: model 1 includes age, race/ethnicity, gender, diabetes mellitus, hypertension, hypercholesterolemia, LDH cholesterol, and current smoking, model 2 includes all factors in model 1 and CRP, model 3 includes all factors in model 2 and chronic kidney disease, and model 4 includes all factors in model 3 and NT-proBNP

ULSAM: model includes age, current smoking, BMI, systolic blood pressure, antihypertensive treatment, total cholesterol, HDL cholesterol, lipid-lowering treatment, diabetes treatment and previous or prevalent cancer

AGES-R: model includes age, diabetes mellitus, BMI, hypertension medication, systolic blood pressure, smoking, CRP, creatinine, total cholesterol, HDL cholesterol, triglycerides and statin medication

FINRISK: model includes total cholesterol, HDL cholesterol, systolic blood pressure, hypertension medication, diabetes mellitus, current smoking and region of Finland

HUNT: model includes gender, age, total cholesterol, HDL cholesterol, systolic blood pressure, treatment of hypertension, smoking, diabetes mellitus, eGFR, history of myocardial infarction and CRP

PIVUS: model 1 includes gender, hypertension, diabetes mellitus, HDL cholesterol, LDL cholesterol, current smoking, BMI, eGFR, CRP, and previous cardiovascular disease, model 2 includes all factors in model 1 and NT-proBNP, and model 3 includes the Framingham risk score

SSHEC: model includes age, gender, area, family history, diabetes mellitus, blood pressure, total cholesterol and HDL cholesterol 


\section{General discussion}
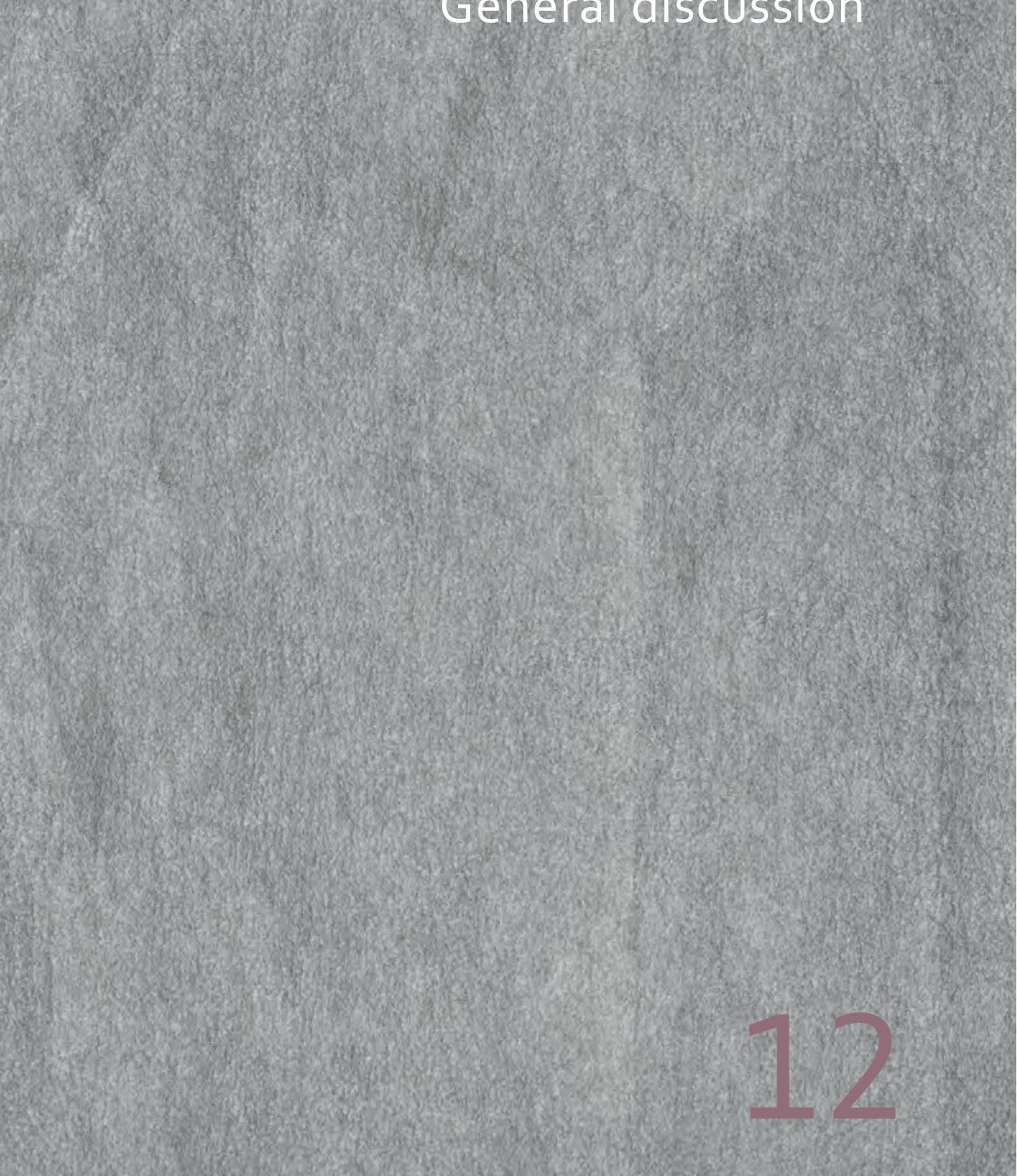
The diagnosis of acute myocardial infarction has undergone a noticeable transformation since the introduction of the high-sensitivity cardiac troponin assays ${ }^{1,2}$. The increased sensitivity of the assays empowered the detection of low levels and small changes in cardiac troponins and was therefore at the basis of the development of more accurate and faster diagnostic algorithms ${ }^{3-6}$. However, at the same time, the detection of previously unnoticed, slightly elevated cardiac troponin levels in various populations without acute cardiac injury complicated the interpretation of cardiac troponin concentrations ${ }^{7}$.

This thesis highlights four aspects associated with the complexity of interpretation of cardiac troponins in the high-sensitivity era (discussed in the following chapters): First, the variation in cardiac troponin concentrations over the day (chapters 3 and 4). Second, the effect of impaired renal function on cardiac troponin levels (chapters 5 and 6). Third, the differences between CTnT and CTnl (chapters 7 and 8). Fourth, persistently elevated basal cardiac troponin concentrations and their clinical significance (chapters 9, 10 and 11).

In this discussion section, we shine light on the four major findings that are described in this thesis and provide directions towards further research.

\section{Cardiac troponin $\mathrm{T}$, but not cardiac troponin I, exhibits a diurnal rhythm}

The first major finding is the circadian rhythm in basal cTnT, but not in cTnl, concentrations (chapters 2, 3, 4, and 5) 8,9. This rhythm is characterized by gradually decreasing concentrations throughout daytime and rising concentrations during night-time ${ }^{10}$.

The presence of a diurnal rhythm implies that both the absolute concentrations and the changes in serially measured cTnT are of different magnitude at different time points. Since the current guidelines presume random variation in cardiac troponin levels and apply the same cut-off values during the whole day ${ }^{2,11}$, the diurnal rhythm may affect the diagnosis of acute myocardial infarction. Our results from chapter 3 , in which we examined the diagnostic accuracy during the high- (early morning) and low-level phase (evening), show that the diagnostic accuracy is not time-dependent, and indicate that the circadian rhythm in cTnT does not affect diagnosis of acute myocardial infarction. Nevertheless, the current approach 
used to analyze the effect of the diurnal rhythm on the diagnosis of acute myocardial infarction by examining diagnostic accuracy, does not allow us to draw any conclusions on whether the optimal cut-off values are identical throughout the day. Future, extended or pooled, cohort studies establishing the sensitivity and specificity of different cut-off values at different time-intervals, may help us to determine whether cut-off values for CTnT should be time-dependent.

\section{Decreased renal elimination is not the main driver}

In addition to the possible clinical implications of the diurnal variation, we employed it as a model to examine the effect of impaired renal clearance on cTnT concentrations (chapter 5). At the basis of this study was the hypothesis that if impaired renal elimination and subsequent accumulation, and not increased cardiac release, are the main drivers behind elevated cardiac troponin release, this would affect the diurnal rhythm in cTnT concentrations: A prolongation of the terminal half-life of cTnT will fade the circadian rhythm. This hypothesis was supported by a mathematical model ${ }^{12}$. In contrast, we demonstrated that the circadian rhythm remains similar in subjects with severe chronic kidney disease, and therefore we showed that impaired renal elimination is not the main driver behind elevated cTnT concentrations in subjects with impaired renal function.

Our conclusion is in line with the results of several cross-sectional studies indicating the multi-etiology of cardiac troponin elevations, including factors that are associated with myocardial injury ${ }^{13,14}$. However, stronger support for our findings is provided by longitudinal cohort studies that show a robust association between basal elevated cardiac troponin concentrations and premature mortality and morbidity. The fact that these associations are almost unaffected by adjustment for renal function indicate that cardiac troponin levels rather reflect an underlying risk profile or (subclinical) disease, rather than a mere consequence of impaired renal elimination ${ }^{15-18}$.

In conclusion, we should take elevated cardiac troponin concentrations seriously as an indicator for underlying cardiovascular disease and future risk. Our results therefore advocate for an extensive diagnostic work-up, not limited to the rule-out of acute myocardial 
infarction (based on (adjusted) diagnostic algorithms ${ }^{19}$ ), in all patients with elevated cardiac troponin concentrations irrespective of their estimated glomerular filtration rate ${ }^{13}$.

We cannot deny that the conclusion that decreased renal elimination is not the main driver behind persistently elevated cTnT levels in subjects with impaired renal function might feel contra intuitive considering the successful identification of cTnT fragments in the urine of patients suffering acute myocardial injury (chapter 6). However, it is important to consider that both studies examine two distinct settings. The presence of cardiac troponin fragments in urine in the acute setting has only been detected in patients with concomitant proteinuria ${ }^{20}$. Furthermore, it is important to bear in mind that the finding that impaired renal clearance is not the main driver behind elevated CTnT levels, does not exclude that cardiac troponin (fragments) may, in part, be removed from the human body by renal clearance $21-23$.

\section{Combining cardiac troponin $\mathrm{T}$ and cardiac troponin I increases early rule-out}

The third major finding involves the early diagnosis of acute myocardial infarction using a combination of cTnT and cTnl concentrations. In chapter 8, we demonstrated how combining cardiac troponin levels, both mathematical combinations and a combination algorithm, leads to a three-to-six-fold increase in the number of patients in whom acute myocardial infarction can be ruled-out after a single blood draw at presentation compared to the current algorithm (based on either cTnT or cTnI) ${ }^{2}$. This may have major impact on clinical practice since it allows more efficient patient care and resource allocation ${ }^{24-29}$.

The addition of a second biomarker to cardiac troponin for the rapid rule-in and rule-out of acute myocardial infarction may increase the specificity of a single measurement at presentation and can be considered as a next step towards increasingly accurate and fast clinical decision making ${ }^{25,30-35}$. Nevertheless, we should be aware of the fact that dual testing can also complicate interpretation and may be associated with logistic challenges. Since none of the current diagnostic platforms offers high-sensitivity cTnT and cTnl assays, a direct, broad and prompt implementation of this combination strategy is not (yet) achievable. 
A second point that merits attention, is the performance of the diagnostic algorithms in the external validation cohort (chapter 8): Despite the high negative predictive value (99.4 $99.6 \%)$, the number of false-negative findings (0.8-1.5\%) is increased in comparison with the internal cohort. It is a common phenomenon that algorithms show different performances in distinct cohorts, and it is for example also the case for the 0-hour/1-hour algorithm ${ }^{36}$. Nevertheless, it challenges us to reflect on the underlying mechanisms (different patient characteristics, distinct allocation strategies, analytical variation in the lower range and the outlier-driven approach of determining cut-off values ${ }^{37-39}$ ), and to consider future research strategies like recalibration of cut-off values in even bigger datasets and specific subgroup analyses ${ }^{39-41}$.

\section{Basal cardiac troponin levels predict premature mortality}

Fourth and final major finding is the prognostic value of basal cardiac troponin values in the general population. We performed a meta-analysis, with data on 65019 participants from 11 cohort studies, in which we showed a significant relationship between basal cardiac troponin levels, also below the $99^{\text {th }}$ percentile, and all-cause and cardiovascular mortality during follow-up (chapter 11) (2) $^{2}$ Our data corroborate and extend the findings of previous studies $^{43,44}$.

The finding that cardiac troponin levels correlate to prognosis in asymptomatic individuals, raises the question whether and how baseline cardiac troponin concentrations are of additional value in cardiovascular risk management ${ }^{14}$. Chapter 11 shows that the addition of cardiac troponins leads to a significant, but numerically small improvement of existing prediction models ${ }^{42}$. Nevertheless, remaining questions merit attention: First, do changes in cardiac troponin concentrations translate directly into risk prediction? Second, may interventions affect both the outcome and the basal concentration of cardiac troponins? Recent studies indicate that changes in cardiac troponin levels over time are reflected in the prognosis ${ }^{45-49}$. Furthermore, whereas exercise training interventions did not affect basal cardiac troponin levels (chapters 9 and 10)50,51, other interventions like statin therapy (modestly) reduced both cTnl concentrations and associated risk ${ }^{52,53}$. Notwithstanding, 
additional studies towards the effect of possible risk stratification strategies - including the target population, biomarker panel and timing - on health related and economic outcomes seem to be necessary prior to implementation $45,49,54-57$.

\section{From state of the art to the future}

This thesis paid attention to multiple aspects of cardiac troponins in acute and chronic conditions. Based on our main findings, we propose a number of future directions. Besides, the ultimate challenge for the upcoming years seems to be finding a solution for the limited specificity of cardiac troponin elevations for the diagnosis of acute myocardial infarction.

Three future strategies may help us to increase the specificity of cardiac troponin testing for acute myocardial infarction: The first strategy involves the optimization of interpretation of cardiac troponin concentrations measured with the current assays. By determining adjusted, optimal cut-off values for factors like time of presentation, gender, age or previous concentrations ${ }^{58,59}$, cardiac troponin testing may become more specific to detect acute myocardial infarction. The second strategy relates to chapter 8 of this thesis: The specificity of cardiac troponin testing may increase by addition of a second cardiac or ischemic biomarker to the diagnostic algorithm ${ }^{25,30-34}$. The third, and most challenging strategy is the development of new, high-specificity (cardiac troponin) assays for the diagnosis of acute myocardial infarction. Over the last decades, our research group has partially solved the puzzle on release, degradation, and elimination by unraveling the fragmentation of cardiac troponins ${ }^{60-62}$. A next step may involve the identification of cardiac troponin forms that are specific for acute myocardial infarction, and can be targeted by new assays. 


\section{References}

1. Thygesen K, Alpert JS, Jaffe AS, et al. Third universal definition of myocardial infarction. Eur Heart J 2012;33:255167.

2. Roffi M, Patrono C, Collet JP, et al. 2015 ESC Guidelines for the management of acute coronary syndromes in patients presenting without persistent ST-segment elevation: Task Force for the Management of Acute Coronary Syndromes in Patients Presenting without Persistent ST-Segment Elevation of the European Society of Cardiology (ESC). Eur Heart J 2016;37:267-315.

3. Reichlin T, Hochholzer W, Bassetti S, et al. Early diagnosis of myocardial infarction with sensitive cardiac troponin assays. N Engl J Med 2009;361:858-67.

4. Keller T, Zeller T, Peetz D, et al. Sensitive troponin I assay in early diagnosis of acute myocardial infarction. N Engl J Med 2009;361:868-77.

5. Jaeger $C$, Wildi K, Twerenbold R, et al. One-hour rule-in and rule-out of acute myocardial infarction using highsensitivity cardiac troponin I. Am Heart J 2016;171:92-102 e1-5.

6. Reichlin T, Schindler C, Drexler B, et al. One-hour rule-out and rule-in of acute myocardial infarction using highsensitivity cardiac troponin T. Arch Intern Med 2012;172:1211-8.

7. de Lemos JA. Increasingly sensitive assays for cardiac troponins: a review. JAMA 2013;309:2262-9.

8. Klinkenberg LJ, Wildi K, van der Linden N, et al. Diurnal Rhythm of Cardiac Troponin: Consequences for the Diagnosis of Acute Myocardial Infarction. Clin Chem 2016;62:1602-11.

9. van der Linden N, Cornelis T, Klinkenberg LJ, et al. Strong diurnal rhythm of troponin T, but not troponin I, in a patient with renal dysfunction. Int J Cardiol 2016;221:287-8.

10. Klinkenberg LJ, van Dijk JW, Tan FE, van Loon LJ, van Dieijen-Visser MP, Meex SJ. Circulating cardiac troponin T exhibits a diurnal rhythm. J Am Coll Cardiol 2014;63:1788-95.

11. Amsterdam. 2014 ACC/AHA Guideline for the Management of Patients With Non-ST-Elevation Acute Coronary Syndromes: A Report of the American College of Cardiology/American Heart Association Task Force on Practice Guidelines (vol 130, pg e344, 2014). Circulation 2014;130:E433-E4.

12. Luck S, Thurley K, Thaben PF, Westermark PO. Rhythmic degradation explains and unifies circadian transcriptome and proteome data. Cell Rep 2014;9:741-51.

13. Irfan A, Twerenbold $R$, Reiter $M$, et al. Determinants of high-sensitivity troponin $T$ among patients with a noncardiac cause of chest pain. Am J Med 2012;125:491-8 e1.

14. Rubin J, Matsushita K, Lazo M, et al. Determinants of minimal elevation in high-sensitivity cardiac troponin T in the general population. Clin Biochem 2016;49:657-62.

15. Cardinaels EP, Daamen MA, Bekers O, et al. Clinical Interpretation of Elevated Concentrations of Cardiac Troponin T, but Not Troponin I, in Nursing Home Residents. J Am Med Dir Assoc 2015;16:884-91.

16. Oluleye OW, Folsom AR, Nambi V, Lutsey PL, Ballantyne CM, Investigators AS. Troponin T, B-type natriuretic peptide, C-reactive protein, and cause-specific mortality. Ann Epidemiol 2013;23:66-73.

17. Bosselmann H, Egstrup M, Rossing K, et al. Prognostic significance of cardiovascular biomarkers and renal dysfunction in outpatients with systolic heart failure: a long term follow-up study. Int J Cardiol 2013;170:202-7.

18. Scheven L, de Jong PE, Hillege HL, et al. High-sensitive troponin T and N-terminal pro-B type natriuretic peptide are associated with cardiovascular events despite the cross-sectional association with albuminuria and glomerular filtration rate. Eur Heart J 2012;33:2272-81.

19. Twerenbold R, Wildi K, Jaeger C, et al. Optimal Cutoff Levels of More Sensitive Cardiac Troponin Assays for the Early Diagnosis of Myocardial Infarction in Patients With Renal Dysfunction. Circulation 2015;131:2041-50.

20. Gosling P, Hughes EA, Reynolds TM, Fox JP. Microalbuminuria is an early response following acute myocardial infarction. Eur Heart J 1991;12:508-13.

21. Diris JH, Hackeng CM, Kooman JP, Pinto YM, Hermens WT, van Dieijen-Visser MP. Impaired renal clearance explains elevated troponin T fragments in hemodialysis patients. Circulation 2004;109:23-5. 
22. Cardinaels EP, Mingels AM, van Rooij T, Collinson PO, Prinzen FW, van Dieijen-Visser MP. Time-dependent degradation pattern of cardiac troponin T following myocardial infarction. Clin Chem 2013;59:1083-90.

23. Friden V, Starnberg K, Muslimovic A, et al. Clearance of cardiac troponin T with and without kidney function. Clin Biochem 2017.

24. Shortt C, Xie F, Whitlock R, et al. Economic Considerations of Early Rule-In/Rule-Out Algorithms for The Diagnosis of Myocardial Infarction in The Emergency Department Using Cardiac Troponin and Glycemic Biomarkers. Clin Chem 2017;63:593-602.

25. Shortt C, Ma J, Clayton N, et al. Rule-In and Rule-Out of Myocardial Infarction Using Cardiac Troponin and Glycemic Biomarkers in Patients with Symptoms Suggestive of Acute Coronary Syndrome. Clin Chem 2017;63:40314.

26. Chapman AR, Shah AS, Mills NL. Appropriate Use of High-Sensitivity Cardiac Troponin Levels in Patients With Suspected Acute Myocardial Infarction. JAMA Cardiol 2017;2:228.

27. Sandoval Y, Smith SW, Shah AS, et al. Rapid Rule-Out of Acute Myocardial Injury Using a Single High-Sensitivity Cardiac Troponin I Measurement. Clin Chem 2017;63:369-76.

28. Goodacre S, Cross E, Arnold J, Angelini K, Capewell S, Nicholl J. The health care burden of acute chest pain. Heart 2005;91:229-30.

29. Goodacre S, Thokala P, Carroll C, et al. Systematic review, meta-analysis and economic modelling of diagnostic strategies for suspected acute coronary syndrome. Health Technol Assess 2013;17:v-vi, 1-188.

30. Stallone F, Schoenenberger AW, Puelacher C, et al. Incremental value of copeptin in suspected acute myocardial infarction very early after symptom onset. Eur Heart J Acute Cardiovasc Care 2016;5:407-15.

31. Schaub N, Reichlin T, Twerenbold R, et al. Growth differentiation factor-15 in the early diagnosis and risk stratification of patients with acute chest pain. Clin Chem 2012;58:441-9.

32. Haaf $P$, Twerenbold $R$, Reichlin $T$, et al. Mid-regional pro-adrenomedullin in the early evaluation of acute chest pain patients. Int J Cardiol 2013;168:1048-55.

33. Reiter M, Twerenbold R, Reichlin $\mathrm{T}$, et al. Heart-type fatty acid-binding protein in the early diagnosis of acute myocardial infarction. Heart 2013;99:708-14.

34. Haaf P, Zellweger C, Reichlin T, et al. Utility of C-terminal proendothelin in the early diagnosis and risk stratification of patients with suspected acute myocardial infarction. Can J Cardiol 2014;30:195-203.

35. Nestelberger T, Wildi K, Boeddinghaus J, et al. Characterization of the observe zone of the ESC 2015 highsensitivity cardiac troponin $0 \mathrm{~h} / 1 \mathrm{~h}$-algorithm for the early diagnosis of acute myocardial infarction. Int J Cardiol 2016;207:238-45.

36. Pickering JW, Greenslade JH, Cullen L, et al. Assessment of the European Society of Cardiology 0-Hour/1-Hour Algorithm to Rule-Out and Rule-In Acute Myocardial Infarction. Circulation 2016;134:1532-41.

37. Mueller C, Roffi M, Task Force of the ESCGftMoACSiPPWPSTSE. Letter by Mueller and Roffi Regarding Article, "Assessment of the European Society of Cardiology 0-Hour/1-Hour Algorithm to Rule-Out and Rule-In Acute Myocardial Infarction". Circulation 2017;135:e921-e2.

38. Than MP, Pickering JW, Cullen L, Kavsak PA. Response by Than et al to Letter Regarding Article, "Assessment of the European Society of Cardiology 0-Hour/1-Hour Algorithm to Rule-Out and Rule-In Acute Myocardial Infarction". Circulation 2017;135:e923-e4.

39. Pickering JW, Than MP. The small number problem in diagnostic algorithms and why we need to bootstrap. Clin Biochem 2017.

40. Rubini Gimenez M, Twerenbold R, Reichlin T, et al. Direct comparison of high-sensitivity-cardiac troponin I vs. T for the early diagnosis of acute myocardial infarction. Eur Heart J 2014;35:2303-11.

41. Slagman A, Searle J, Vollert JO, et al. Sex differences of troponin test performance in chest pain patients. Int J Cardiol 2015;187:246-51.

42. van der Linden N, Klinkenberg $\sqcup$, Bekers O, et al. Prognostic value of basal high-sensitive cardiac troponin levels on mortality in the general population: A meta-analysis. Medicine (Baltimore) 2016;95:e5703. 
43. Blankenberg S, Salomaa V, Makarova N, et al. Troponin I and cardiovascular risk prediction in the general population: the BiomarCaRE consortium. Eur Heart J 2016;37:2428-37.

44. Sze J, Mooney J, Barzi F, Hillis GS, Chow CK. Cardiac Troponin and its Relationship to Cardiovascular Outcomes in Community Populations - A Systematic Review and Meta-analysis. Heart Lung Circ 2016;25:217-28.

45. Hughes MF, Ojeda F, Saarela O, et al. Association of Repeatedly Measured High-Sensitivity-Assayed Troponin I with Cardiovascular Disease Events in a General Population from the MORGAM/BiomarCaRE Study. Clin Chem 2017;63:334-42.

46. Eggers KM, Venge P, Lindahl B, Lind L. Cardiac troponin I levels measured with a high-sensitive assay increase over time and are strong predictors of mortality in an elderly population. J Am Coll Cardiol 2013;61:1906-13.

47. deFilippi CR, de Lemos JA, Christenson RH, et al. Association of serial measures of cardiac troponin $\mathrm{T}$ using a sensitive assay with incident heart failure and cardiovascular mortality in older adults. JAMA 2010;304:2494-502.

48. Eggers KM, Kempf T, Larsson A, et al. Evaluation of Temporal Changes in Cardiovascular Biomarker Concentrations Improves Risk Prediction in an Elderly Population from the Community. Clin Chem 2016;62:485-93. 49. McEvoy JW, Chen Y, Ndumele CE, et al. Six-Year Change in High-Sensitivity Cardiac Troponin T and Risk of Subsequent Coronary Heart Disease, Heart Failure, and Death. JAMA Cardiol 2016;1:519-28.

50. van der Linden $\mathrm{N}$, Tieland $\mathrm{M}$, Klinkenberg $\amalg$, et al. The effect of a six-month resistance-type exercise training program on the course of high sensitive cardiac troponin T levels in (pre)frail elderly. Int J Cardiol 2014;175:374-5.

51. van der Linden $N$, Klinkenberg $L$, Leenders $M$, et al. The effect of exercise training on the course of cardiac troponin T and I levels: three independent training studies. Sci Rep 2015;5:18320.

52. White HD, Tonkin A, Simes J, et al. Association of contemporary sensitive troponin I levels at baseline and change at 1 year with long-term coronary events following myocardial infarction or unstable angina: results from the LIPID Study (Long-Term Intervention With Pravastatin in Ischaemic Disease). J Am Coll Cardiol 2014;63:345-54.

53. Tonkin AM, Blankenberg S, Kirby A, et al. Biomarkers in stable coronary heart disease, their modulation and cardiovascular risk: The LIPID biomarker study. Int J Cardiol 2015;201:499-507.

54. Sandoval Y, Herzog CA, Love SA, et al. Prognostic Value of Serial Changes in High-Sensitivity Cardiac Troponin I and T over 3 Months Using Reference Change Values in Hemodialysis Patients. Clin Chem 2016;62:631-8.

55. Kawahara C, Tsutamoto T, Sakai H, et al. Prognostic value of serial measurements of highly sensitive cardiac troponin I in stable outpatients with nonischemic chronic heart failure. Am Heart J 2011;162:639-45.

56. Masson S, Anand I, Favero C, et al. Serial measurement of cardiac troponin T using a highly sensitive assay in patients with chronic heart failure: data from 2 large randomized clinical trials. Circulation 2012;125:280-8.

57. de Lemos JA, Ayers CR, Levine BD, et al. A Multimodality Strategy for Cardiovascular Risk Assessment: Performance in Two Population-Based Cohorts. Circulation 2017.

58. Kimenai DM, Henry RM, van der Kallen CJ, et al. Direct comparison of clinical decision limits for cardiac troponin T and I. Heart 2016;102:610-6.

59. Shah ASV, Ferry AV, Mills NL. Cardiac Biomarkers and the Diagnosis of Myocardial Infarction in Women. Curr Cardiol Rep 2017;19:40.

60. Mingels AM, Cardinaels EP, Broers NJ, et al. Cardiac Troponin T: Smaller Molecules in Patients with End-Stage Renal Disease than after Onset of Acute Myocardial Infarction. Clin Chem 2017;63:683-90.

61. Streng AS, de Boer D, van Doorn WP, et al. Identification and Characterization of Cardiac Troponin T Fragments in Serum of Patients Suffering from Acute Myocardial Infarction. Clin Chem 2017;63:563-72.

62. Streng AS, de Boer D, Bouwman FG, et al. Development of a targeted selected ion monitoring assay for the elucidation of protease induced structural changes in cardiac troponin T. J Proteomics 2016;136:123-32. 
Cardiac Troponins: State of the (He)Art 



\section{Summary}

Cardiac troponins, cardiac troponin $\mathrm{T}$ (cTnT) and cardiac troponin I (cTnl), are the biochemical cornerstones in the diagnosis of acute myocardial infarction without characteristic changes on the electrocardiogram (ECG). The recent introduction of the high-sensitivity cardiac troponin assays was at the basis of the increasingly accurate and rapid clinical decision making in patients with acute chest pain. Nevertheless, the increased sensitivity of the recent assays has not only expedited diagnosis of acute myocardial infarction, but also resulted in the detection of, previously undetectable, low cardiac troponin concentrations in subjects without acute myocardial infarction. The aim of this thesis is to increase our understanding of basal cardiac troponin levels in non-acute conditions, their impact on the diagnostics of acute myocardial infarction, and how these aspects can be used to improve the interpretation of cardiac troponins levels.

Chapter 1 provides a general introduction on cardiac troponins and the diagnosis of acute myocardial infarction. Furthermore, it describes the prevalence and prognostic value of persistently elevated cardiac troponin concentrations.

Chapter 2 illustrates the impact of chronically elevated cardiac troponin concentrations with a case. This case highlights four aspects that are discussed in this thesis (discussed in the following chapters): First, the variation in cardiac troponin concentrations over the day (chapters 3 and 4). Second, the effect of impaired renal function on cardiac troponin levels (chapters 5 and 6). Third, the differences between CTnT and CTnl (chapters 7 and 8). Fourth, persistently elevated basal cardiac troponin concentrations and their clinical significance (chapters 9, 10 and 11).

Since increased cardiac troponin concentrations are not specific for the presence of an acute myocardial infarction, serial measurement of cardiac troponins is of profound importance in diagnostics. An important assumption for the interpretation of these serial cardiac troponin concentrations is the random variation of concentrations in clinically stable conditions. In chapters 3 and 4, we demonstrate that this assumption holds true for cTnl, but not for cTnT. Specifically, in chapter 3 , which contains the results of a biological variation study with hourly sampling during day and night in 24 patients without chronic kidney disease, we show an 
omnipresent diurnal rhythm in CTnT. This rhythm is characterized by gradually decreasing concentrations during the day and increasing concentrations of cTnT during night-time. Since the diurnal pattern in CTnT concentrations may cause increases or decreases in serial measurements to be more likely in certain timeframes, we additionally evaluated the effect of time on the diagnostic accuracy in an international multicenter diagnostic trial (2782 patients with acute chest pain). This evaluation shows a very high and time-frame independent diagnostic accuracy of cTnT for the diagnosis of acute myocardial infarction. Nevertheless, in early presenters ( $\leq 3 \mathrm{~h}$ after the onset of chest pain) the effect of the timeframe seems to be more pronounced and leads to a borderline significant decrease in diagnostic accuracy for cTnT concentrations at one hour after presentation and for the combination of CTnT concentration at presentation combined with the absolute change within the first hour. Therefore, the diurnal rhythm may provide an explanation in cases where the clinical assessment is discordant with the biochemical kinetics. In addition, this chapter shows random variation in cTnl levels over the day without an effect on the diagnostic accuracy of cTnl. Moreover, chapter 4 focuses on the variation in cTnl levels during day and night. In addition to the population described in chapter 3, we included 20 subjects with chronic kidney disease. Their baseline cTnl levels were slightly higher and accompanied by a significant lower analytical coefficient of variation compared to the subjects without chronic kidney disease. Nevertheless, we observed a similar random variation in cTnl levels over the day in both groups.

Persistently elevated cardiac troponin concentrations are highly prevalent among subjects with impaired renal function. A better understanding and interpretation of these elevations, requires more insight in the underlying mechanism: Is it the heart, by increased release, or the kidneys, by decreased renal elimination? Chapters 5 and 6 consider the role of the kidneys in the terminal elimination of cTnT from the circulation. Chapter 5 builds upon the previous chapters and employs the diurnal oscillation pattern of cTnT as a model to assess the contribution of impaired renal elimination to persistently elevated cTnT levels in in patients with chronic kidney disease. Mathematically modeling shows that impaired elimination of cTnT should lead to a faded circadian rhythm characterized by a substantial 
decrease in amplitude. Nevertheless, the pattern and the amplitude of the circadian rhythm in CTnT is similar in subjects with or without chronic kidney disease. These observations strongly suggest that other factors, such as increased release of cardiac troponins from the heart due to (subclinical) myocardial injury, are responsible for persistently elevated cardiac troponin levels in subjects with decreased renal function. Whereas the previous chapter focuses on the elimination of cTnT in a chronic setting, chapter 6 examines the elimination of cTnT in patients suffering from acute myocardial infarction. We are the first to develop and validate a targeted mass spectrometry assay for the detection of cTnT (fragments) in urine. Subsequently, we show the presence of cTnT fragments in a subset of patients. Nevertheless, whether the presence of CTnT in urine reflects a physiological or rather a pathophysiological process needs to be elucidated.

cTnT and cTnl are both proteins from the same complex inside the cardiomyocytes and are equally recommended for the diagnosis of acute myocardial infarction. Although there are some clear differences between both cardiac troponins, which are further highlighted in chapters 7 and 8 . In chapter 7, we show a substantial variation in CTnT concentrations in both serum and plasma of different individuals after the addition of a standardized amount of cardiac troponin. This evident variation is not present for other cardiac biomarkers (cTnl, creatine kinase $\mathrm{MB}$ isoenzyme and myoglobine) and disappears when the experiment is replicated in deproteinated serum. These findings indicate the presence of one or more factors that have specific interaction with cTnT and not with cTnl. Additional studies have to elucidate whether this effect can also be found in vivo and what its exact clinical implications are. Chapter 8 examines the clinical impact of the use of the different cardiac troponins for the diagnosis of acute myocardial infarction and evaluates whether combining them might overcome individual limitations. When applying the current 0-hour/1-hour algorithm for cTnT or for cTnl in an international multicenter center diagnostic trial (2225 patients with acute chest pain), the agreement on the early diagnosis of acute myocardial infarction is moderate and the rule-out of patients without an acute myocardial infarction at presentation is limited. The combination of cTnT and cTnl for the rule-out of acute myocardial infarction leads to a significant increase in the number of safe rule-outs after a single blood draw. These 
results are supported by the validation of combination strategies in an external cohort (2539 patients). Nevertheless, some limitations of this strategies merit attention: First, the 3-to-6fold increase in rule-outs at presentation due to combining cTnT and cTnl seems to lead to a very small, but inevitable increase in the absolute number of false-negative results. Second, the clinical implementation of this dual-marker approach would currently be associated with substantial logistic obstacles.

The final part of this thesis reflects on persistently elevated cardiac troponin concentrations that are common in various subpopulations and associated with an unfavorable prognosis. Chapters 9 and 10 explore the effects of exercise training, a preventive lifestyle intervention, on the course of basal cardiac troponin levels. In chapter 9, we analyze the effects of a 24week resistance-type exercise training program on the cTnT levels of (pre)frail elderly. There is no significant difference in the course of cTnT levels between the intervention ( 25 subjects) and the control group (27 subjects). A limitation of this study might be the limited (absolute) training load due to the frailty of the subjects. Furthermore, the effect on cTnl concentrations is unclear. To overcome these limitations, chapter 10 presents two additional exercise training studies (including 13 and 39 subjects) and an additional analysis of cardiac cTnl in the previous study. Despite the beneficial changes in metabolic profile and physical performance, there is also no effect on circulating cardiac troponins levels in these studies. To quantify the relationship between basal cardiac troponin levels and prognosis in the general population, we perform a meta-analysis in Chapter 11. This meta-analysis with data on 65019 participants from 11 cohort studies shows a robust association of basal cardiac troponin levels with all-cause and cardiovascular mortality during follow-up in the general population.

Lastly, chapter 12, contains a general discussion of the work presented in this thesis and provides directions for future research. 


\section{Samenvatting}

Cardiale troponines, cardiaal troponine $\mathrm{T}(\mathrm{cTnT})$ en cardiaal troponine I (cTnl), zijn de biochemische pijlers in de diagnostiek van een acuut myocardinfarct in afwezigheid van kenmerkende veranderingen op het elektrocardiogram (ECG). Door de recent geïntroduceerde hoog-sensitieve cardiale troponinetesten, kunnen infarcten steeds accurater en sneller worden gediagnosticeerd bij patiënten die zich melden met pijn op de borst. De hoge sensitiviteit zorgt er echter ook voor dat er regelmatig (licht) verhoogde concentraties worden gevonden bij personen die geen acuut myocardinfarct hebben. Het doel van dit proefschrift is om deze niet-acute verhogingen verder in kaart te brengen, te bestuderen hoe zij kunnen interfereren met de diagnostiek en uiteindelijk te onderzoeken of deze kennis ons kan helpen om cardiale troponineconcentraties beter te interpreteren.

Hoofdstuk 1 vormt de introductie van dit proefschrift. Het geeft een korte beschrijving van de diagnostiek van het acute myocardinfarct en de rol die cardiale troponines hierin spelen. Daarnaast worden in dit hoofdstuk chronische troponineverhogingen en hun prognostische implicaties besproken.

Hoofdstuk 2 illustreert de impact van chronisch verhoogde cardiale troponines aan de hand van een casus. In deze casus komen vier aspecten aan de orde die verder in dit proefschrift aan bod komen: Ten eerste, de schommelingen in cardiale troponineconcentraties over de dag (hoofdstuk 3 en 4). Ten tweede, de mogelijke effecten van de nieren op cardiale troponines (hoofdstuk 5 en 6). Ten derde, de verschillen tussen cTnT en cTnl (hoofdstuk 7 en 8). Ten vierde, de prognostische betekenis van chronische verhoogde cardiale troponineconcentraties (hoofdstuk 9, 10 en 11).

Omdat verhoogde cardiale troponineconcentraties niet specifiek zijn voor de aanwezigheid van een acuut myocardinfarct, is het serieel meten van licht verhoogde cardiale troponineconcentraties van belang bij de diagnostiek. Een belangrijke aanname bij de interpretatie van seriële waarden is dat de concentraties onder normale omstandigheden (dus in de afwezigheid van een infarct) willekeurig schommelen over de dag. In hoofdstuk 3 en 4 laten we zien dat deze aanname klopt voor cTnl, maar niet voor cTnT. Hoofdstuk 3 rapporteert de resultaten van een biologische variatie studie waarbij gedurende een etmaal bij 24 proefpersonen ieder uur bloed is afgenomen. Hierbij vertonen alle proefpersonen een 
duidelijk dag-nacht ritme in cTnT: Over de dag neemt de concentratie cTnT in het bloed langzaam af en gedurende de nacht neemt deze weer toe. Het dag-nacht ritme zou ertoe kunnen leiden dat (grote) verschillen in serieel gemeten waarden op sommige tijdstippen waarschijnlijker zijn dan op andere tijdstippen en dat hiermee het vermogen om onderscheid te maken tussen wel of geen infarct tijdsafhankelijk is. Daarom hebben we in dit hoofdstuk tevens gekeken of het diagnostische vermogen van cardiale troponines afhankelijk is van het moment van de dag. Dit hebben we gedaan in een internationale diagnostische multicenter studie (2782 patiënten met acute pijn op de borst). Uit deze analyse blijkt dat het vermogen van cTnT om patiënten met en zonder een infarct te onderscheiden hoog is, en niet lijkt te worden gestoord door het dag-nacht ritme. Echter, in de groep die zich vroeg presenteert ( $\leq$ 3 uur na het ontstaan van pijn op de borst) zien we dat het diagnostisch vermogen van cTnT beter lijkt in de avonduren (dit verschil is significant wanneer alleen gekeken wordt naar de concentratie na een uur of naar het verschil in seriële metingen en bijna significant als dit wordt gecombineerd met de meting bij presentatie). Dit zou mogelijk kunnen verklaren waarom bij sommige patiënten de klinische presentatie niet overeenkomt met het beeld in de laboratoriumuitslag. Daarnaast laat dit hoofdstuk zien dat cTnI concentraties wél random fluctueren gedurende de dag en het moment waarop de patiënt zich presenteert geen effect heeft op het diagnostisch vermogen van de test. In aanvulling hierop focust hoofdstuk 4 zich volledig op de variatie in cTnl concentraties gedurende de dag en de nacht. Hiertoe zijn 20 proefpersonen met een chronische nierziekte toegevoegd aan de populatie zoals deze beschreven is in hoofdstuk 3. De beginconcentraties van cTnl waren significant hoger in deze tweede groep, hetgeen gepaard ging met een lagere analytische variatie. Ondanks dit verschil zien we in beide groepen een even grote, random spreiding van cTnl levels over de dag.

Individuen met een verminderde nierfunctie hebben relatief vaak chronisch verhoogde cardiale troponineconcentraties in hun bloed. Het onderliggende mechanisme voor deze verhogingen is echter nog onopgehelderd. De centrale vraag hierbij is: Komt het door de nieren (doordat zij minder cardiale troponines verwijderen uit het bloed) of komt het door het hart (doordat het meer cardiale troponines afgeeft aan het bloed)? In hoofdstuk 5 en 6 hebben we gekeken naar de rol die de nieren spelen bij het verwijderen van cardiale 
troponines. Hoofdstuk 5 is een vervolg op de voorgaande hoofdstukken en gebruikt het ritme in CTnT om de mate waarin de nieren verantwoordelijk zijn voor de basaal verhoogde troponineconcentraties te onderzoeken. We hebben hierbij allereerst aan de hand van een mathematisch model laten zien dat als er minder CTnT uit het bloed wordt verwijderd bij patiënten met een verminderde nierfunctie, het ritme in cTnT over de dag zal afnemen. Als we echter naar de ritmes in CTnT in de individuen met en in de individuen zonder nierfalen kijken, dan zien we dat het ritme hetzelfde is (ook qua sterkte). Deze bevindingen suggereren dat niet een verminderde uitscheiding door de nieren, maar andere factoren, zoals een verhoogd vrijkomen van cardiale troponines als het resultaat subklinische hartschade, de drijvende krachten zijn achter de troponine verhogingen in individuen met een verminderde nierfunctie. In tegenstelling tot het vorige hoofdstuk richt hoofdstuk 6 zich op de rol van de nieren in de acute setting. In dit hoofdstuk hebben we een methodiek ontworpen en gevalideerd om cTnT middels massaspectrometrie te meten in urine. Vervolgens hebben we hiermee de aanwezigheid van cTnT fragmenten in de urine van een aantal patiënten met een acuut myocardinfarct aangetoond. Het is echter niet duidelijk of de aanwezigheid van troponine in urine een 'normaal', fysiologisch proces is (dat door de lage concentraties niet in ieder monster gemeten kan worden) of dat dit het gevolg is van overloopproteïnurie en enkel bij heel hoge troponineconcentraties in het bloed kan worden gemeten.

cTnT en cTnl zijn twee eiwitten die afkomstig zijn vanuit een gemeenschappelijk eiwitcomplex in de hartspiercel en worden gezien als diagnostische equivalenten (dit wil zeggen; in de huidige richtlijnen worden zij als gelijkwaardige biomarkers gezien). Er zijn echter ook verschillen tussen beide troponines. In hoofdstuk 7 en 8 wordt hier verder bij stilgestaan. In hoofdstuk 7 laten we grote verschillen in de gemeten concentraties CTnT zien als aan het serum of plasma van verschillende individuen dezelfde hoeveelheid cardiaal troponine wordt toegevoegd. Dit is niet het geval voor de andere cardiale biomarkers (cTnl, creatine kinase MB en myoglobine) of als we het serum eerst eiwit-vrij maken. Het lijkt erop dat er één of meerdere factoren in min of meerdere mate aanwezig zijn in het serum en plasma van verschillende individuen en dat deze factoren wel een effect hebben op cTnT, maar niet op cTnl. Vervolgstudies zullen moeten aantonen of dit effect ook in vivo aanwezig is en wat hiervan de klinische implicaties zijn. Hoofdstuk 8 bestudeert welke impact de keuze 
voor het testen van cTnT of cTnl kan hebben en bekijkt of het wellicht beter is om deze testen te combineren. In een internationale multicenter cohortstudie (2225 patiënten met acute pijn op de borst) is de overeenkomst in vroege diagnostiek van het acute myocardinfarct matig als cTnT en cTnl worden vergeleken. Hierbij valt eveneens op dat slechts bij een klein deel van alle patiënten dat geen infarct heeft, een infarct direct na één bloedafname op het moment van presentatie kan worden uitgesloten. Door het combineren van de beide cardiale troponinetesten neemt het aantal patiënten waarbij direct na één bloedafname veilig een infarct kan worden uitgesloten significant toe. Hetzelfde beeld zien we terug als deze combinatiestrategie wordt toegepast in een extern validatie cohort (2539 patiënten met acute pijn op de borst). Kanttekeningen bij deze combinatiestrategieën zijn: 1) de kleine toename in het absolute aantal vals-negatieven (patiënten die voor rule-out in aanmerking komen, maar toch een acuut myocardinfarct blijken te hebben) ten gevolge van een 3-tot-6voudige toename in het aantal vroege rule-outs, en 2 ) het feit dat het meten van twee verschillende cardiale troponines op dit moment in veel ziekenhuizen nog niet mogelijk is vanwege de logistiek.

Het laatste deel van dit proefschrift richt zich op prognostische betekenis van chronische cardiale troponine verhogingen. Hoofdstuk 9 en 10 onderzoeken het effect van levensstijlinterventies, in de vorm van trainingsprogramma's, op cardiale troponineconcentraties over de tijd. In hoofdstuk 9 hebben we gekeken naar het effect van een 24-weeks krachttrainingsprogramma op cTnT concentraties in (pre)fragiele ouderen. Hierbij hebben wij geen verschil kunnen aantonen tussen groep die wel (25 deelnemers) en de groep die geen trainingsprogramma heeft gevolgd (27 deelnemers). Een mogelijke limitatie van deze studie was de beperkte belastbaarheid van de (pre)fragiele deelnemers. Daarnaast laat deze studie in het midden of er mogelijke effecten op cTnl zijn. In hoofdstuk 10 hebben we, rekening houdend met de beperkingen van de vorige studie, de studie uit hoofdstuk 9 verder uitgebreid. Hiertoe hebben we twee extra trainingsstudies toegevoegd (met respectievelijk 13 en 39 deelnemers) en hebben we in alle studies ook cTnl bekeken. Ondanks duidelijke verbeteringen op metabole en fysieke parameters, zagen we ook in deze studie geen effect op cardiale troponineconcentraties. Ten slotte, hebben we in hoofdstuk 11 middels een meta-analyse de relatie tussen basale cardiale troponine levels en het risico 
op vroegtijdige overlijden van mensen in de algemene populatie gekwantificeerd. De uitgevoerde meta-analyse bevat data van 11 cohort studies (in totaal 65019 deelnemers) en laat zien dat verhoogde basale cardiale troponineconcentraties samenhangen met een grotere de kans op vroegtijdig overlijden.

Tot slot bevat hoofdstuk 12 een algemene discussie over het in dit proefschrift beschreven werk en worden suggesties voor vervolgonderzoek aangedragen. 

This chapter describes the (future) valorization of the research described in this thesis. Valorization is thereby defined as the social and economic relevance of research, the target populations of the findings, the related concrete products, services, processes and activities, their innovativeness and future directions ${ }^{1}$.

\section{Relevance}

According to the World Health Organization (WHO), cardiovascular disease, of which acute myocardial infarction is considered as an important entity ${ }^{2}$, is the world's number one killer, responsible for approximately $31 \%$ of all deaths worldwide ${ }^{3}$. Along, cardiovascular disease substantially contributes to disability and decreased quality of life ${ }^{4,5}$. A recent economic evaluation showed that cardiovascular disease accounts for approximately $12 \%$ of the total European health care expenditures ${ }^{6,7}$. However, this may be even an underestimation since the total economic impact on society is bigger due to productivity losses and informal care as result of chronic disease and disability ${ }^{7}$. Urbanization and associated changes in lifestyle at one hand, and longevity at the other hand, will further increase the prevalence of cardiovascular disease in the coming years ${ }^{8,9}$.

This thesis focuses on two ways in which the accurate interpretation of cardiac troponin concentrations may contribute to lower the burden of cardiovascular disease: The distinction of patients with and without myocardial infarction in the acute setting, and the identification of subjects at increased risk for future events based on basal cardiac troponin concentrations.

\section{Target population}

Since this thesis focuses on the optimization of interpretation of cardiac troponin concentrations in both the acute (diagnostic) and chronic (prognostic) setting, its findings are relevant for a large population. For example, whereas the novel insights on combining cardiac troponin T and cardiac troponin I for the early rule-out of acute myocardial infarction refer to emergency department patients, the results on the prognostic value of cardiac troponins are applicable to the general population. 


\section{Products}

As already described in the discussion chapter of this thesis, many of our findings trigger further research. An example of a concept that requires additional research is the application of cardiac troponins for prognosis and prevention. In contrast, a more concrete and innovative concept is the combination of cardiac troponin $\mathrm{T}$ and cardiac troponin I for the diagnosis of acute myocardial infarction.

\section{Implementation of combination testing in clinical practice}

We showed that combining cardiac troponin T and cardiac troponin I in de diagnostic setting is highly promising to overcome the limited specificity of the separate assays. It results in a significant increase in patients in which acute myocardial infarction could be safely ruled-out at presentation (from approximately $10 \%$ to more than 40\%). Therefore, the implementation of this combination strategy may have profound consequences for clinical practice since it may help to overcome unnecessary resource use, overcrowding of the emergency department and anxiety among patients ${ }^{10}$. The fact that high-sensitivity cardiac troponin $T$ and high-sensitivity cardiac troponin I assays do currently not run on the same platform and most laboratories have only one platform running 24/7 for routine measurements is an important obstacle for the introduction of this strategy in clinical practice. The development of (point of care) devices suitable for combined measurement - a project involving diagnostic companies, laboratories and physicians - is therefore crucial. Parallel, we should evaluate the optimal place of combination testing in the diagnostic chain - directly at presentation in the hospital or maybe even earlier at the general practice or in the ambulance setting - , and define optimal algorithms with optimal cut-off values, taking into account the effects of combination testing and the increased number of rule-outs at presentation downstream the diagnostic chain. 


\section{References}

1. Vereniging van Samenwerkende Nederlandse Universiteiten (VSNU). Raamwerk valorisatie-indicatoren. VSNU, 2013. Available at http://www.vsnu.nl/valorisatie-indicatoren.html.

2. Roger VL. Epidemiology of myocardial infarction. Med Clin North Am 2007;91:537-52; ix.

3. World Health Organization (WHO). Factsheet Cardiovascular Diseases (CVDs). WHO, 2016. Available at http://www.who.int/mediacentre/factsheets/fs317/en/.

4. Heyworth IT, Hazell ML, Linehan MF, Frank TL. How do common chronic conditions affect health-related quality of life? Br J Gen Pract 2009;59:e353-8.

5. Ko HY, Lee JK, Shin JY, Jo E. Health-Related Quality of Life and Cardiovascular Disease Risk in Korean Adults. Korean J Fam Med 2015;36:349-56.

6. Tarride JE, Lim M, DesMeules M, et al. A review of the cost of cardiovascular disease. Can J Cardiol 2009;25:e195202.

7. Leal J, Luengo-Fernandez R, Gray A, Petersen S, Rayner M. Economic burden of cardiovascular diseases in the enlarged European Union. Eur Heart J 2006;27:1610-9.

8. Fuster V, Kelly BB, Vedanthan R. Promoting global cardiovascular health: moving forward. Circulation 2011;123:1671-8.

9. Fuster V. Global burden of cardiovascular disease: time to implement feasible strategies and to monitor results. J Am Coll Cardiol 2014;64:520-2.

10. van der Linden N SA, Wodzig, WK BO, Meex SJ Better, higher, lower, faster: increasingly rapid clinical decision making using high-sensitivity cardiac troponin assays. J Lab Precis Med 2017;2:14.

11. Ford I, Shah ASV, Zhang R, et al. High-Sensitivity Cardiac Troponin, Statin Therapy, and Risk of Coronary Heart Disease. Journal of the American College of Cardiology 2016;68:2719-28.

12. White HD, Tonkin A, Simes J, et al. Association of Contemporary Sensitive Troponin I Levels at Baseline and Change at 1 Year With Long-Term Coronary Events Following Myocardial Infarction or Unstable Angina. Journal of the American College of Cardiology 2014;63:345-54.

13. Tonkin AM, Blankenberg S, Kirby A, et al. Biomarkers in stable coronary heart disease, their modulation and cardiovascular risk: The LIPID biomarker study. International Journal of Cardiology;201:499-507. 
Dankwoord

Curriculum Vitae

List of publications 


\section{Dankwoord}

De afgelopen vier jaar waren voor mij veel meer dan het werken aan dit proefschrift: Ik deed unieke ervaringen op (zowel binnen als buiten de wetenschap), leerde veel over mezelf en de wereld waarin ik leef en mocht kennismaken met ontzettend veel inspirerende mensen. Kansen krijgen én grijpen, is iets wat je niet alleen doet. Daarom wil ik de volgende mensen graag bedanken:

Deelnemers aan de diverse studies; jullie medewerking was onmisbaar voor het tot stand komen van dit proefschrift!

Leden van de beoordelingscommissie, dank voor alle moeite en tijd die jullie namen voor het lezen en het beoordelen van mijn proefschrift.

Promotieteam, Prof. Dr. Marja van Dieijen-Visser, Prof. Dr. Luc van Loon en Dr. Steven Meex, jullie vormden het fundament van mijn promotie. Marja, het was een eer om met jou samen te mogen werken. Ik heb enorm veel bewondering voor alles wat je bereikt hebt en hoe je desondanks betrokken kon blijven bij mijn projecten. Dank voor alles! Luc, jij was altijd bereid om mee te denken als dat nodig was. Jouw out-of-the-box ideeën en enthousiasme zijn aanstekelijk. Steven, ik ben jou, als dagelijks begeleider, de meeste dank verschuldigd. Ik had me geen betere en fijnere copromotor kunnen wensen! Qua manier van werken heb ik ontzettend veel van je geleerd, maar het meest dankbaar ben ik je toch wel voor het enorme vertrouwen en de vrijheid die je me altijd gaf. Ik heb hier echt gigantisch veel aan te danken. Bedankt, Steven!

Otto, dank voor het faciliteren van mijn promotie, onze overlegmomenten én de dropjes!

APACE and ADAPT researchers, thanks for sharing your unique data and expertise. It was an honor and a pleasure to be part of the Basel team, to celebrate Kingsday at the CRIB, to be part of the GREAT meeting in Rome, and to swim in the river Rhine. Especially Karin, Christian and John, thanks for all things I have learned from you! 
Graag wil ik een aantal van mijn co-auteurs in het bijzonder bedanken: Michael, jij was betrokken bij mijn eerste twee papers; dank voor deze fijne start! Maurice, het was ontzettend prettig om met jou samen te werken aan de meta-analyse; jouw manier van denken en je razendsnelle begrip maakten grote indruk op mij. Tom, Jeroen en Elizabeth, bedankt voor jullie hulp bij de inclusie van de CKD-patiënten. Jeroen, Stephan en Marijke, klinisch-chemici van de Gelre Ziekenhuizen, dank dat ik altijd bij jullie terecht kon voor metingen. Dear Sarah and Pål your mathematical input in our manuscript on the diurnal rhythm of cardiac troponin T and kidney function was extremely helpful.

Als ik de afgelopen vier jaar iets heb geleerd, dan is het wel dat de mensen waarmee je werkt het grote verschil maken. Daarom wil ik graag ook de overige leden van de onderzoeksgroep bedanken: Alma, als door de wol geverfde troponine-expert was jij onmisbaar om verbanden te leggen en te brainstormen over nieuwe ideeën. Douwe, het was ontzettend leuk en leerzaam om met jou van gedachten te wisselen over zowel onderzoek als doping. Jouw prikdame spelen was voor mij altijd een erebaantje. Will, ik heb je vooral in het laatste jaar leren kennen als iemand met verborgen talenten. Petal, jouw aanwezigheid en verzamelwoede maakten dat ik me geen moment hoefde te vervelen op het lab. Vincent (Fritz), waar was ik zonder jou geweest? Met jou in de buurt kon er voor mijn gevoel (bijna) niets misgaan. Natuurlijk wil ik je ook bedanken voor de memorabele tripjes naar Apeldoorn en Zutphen waarbij jij technische ondersteuning bood en ik me met name focuste op het entertainment. Eline en Lieke, jullie gaven mij het goede voorbeeld (vooral de manier waarop jij, Lieke, alles altijd vlekkeloos regelde) en zorgden ervoor dat ik me gelijk thuis voelde. Dorien, dank voor al jouw epidemiologische input. Ik heb grote bewondering voor de nauwkeurige manier waarop jij iedere keer weer naar mijn manuscripten keek. Judith, succes met de laatste loodjes. Frederique, ik vond het ontzettend leuk om je te helpen en het was fijn om een collega-arts te hebben waarop ik altijd kon terugvallen. Wim en Stephanie, het was prettig om jullie als collega's te hebben; maak er iets moois van de komende jaren! Tenslotte, mijn twee fantastische paranimfen: Sander, wat was ik blij met jou als collega! Qua persoonlijkheid en werkstijl zijn we heel verschillend, maar juist daarom heb ik zoveel van je geleerd! Ik vond het een enorme eer dat ik jouw paranimf mocht zijn. Heel veel succes met 
je opleiding tot klinisch-chemicus! William, pseudo-broertje en opvolger, vanaf het moment dat jij bij ons op de afdeling kwam, maakte je grote indruk met je gedrevenheid, intelligentie en humor. Onze dagelijkse lachmomentjes ga ik zeker missen. Succes met jouw promotie! Mannen, ik vind het echt bijzonder om te midden van jullie mijn proefschrift te mogen verdedigen!

Tijdens mijn promotietraject mocht ik op een ontzettend fijne afdeling werken, waar ik me vanaf begin af aan heel welkom voelde. Ik wil dan ook alle medewerkers (en in het bijzonder ook alle collega-onderzoekers) van het CDL bedanken voor de geweldige tijd die ik hier had. Bedankt voor al jullie hulp en gezelligheid! Daarnaast wil ik ook graag de M3 research unit bedanken voor hun ondersteuning.

Gelukkig hoefde ik me geen moment te vervelen, want snel vond ik een nieuwe uitdaging en leerschool: Collega's van Gupta Strategists bedankt voor het warme welkom en de leerzame en gezellige momenten die ik al met jullie heb mogen delen!

Mijn deelname aan de Nationale DenkTank in 2015 bracht mij ontzettend veel mooie ervaringen. Mede-DenkTankers, Anne, Anke-Marit, Ella, Dorinde, Flora, Fleur, Frank, Gijsbert, Jaco, Job, Jonas, Jorrit, Kay, Koen, Laura, Lisa, Lizan, Milan, Niels, Richard, Rosa, Sarah, Thijmen, de vier maanden dat wij samen deel uit mochten maken van de Nationale DenkTank waren één leerzaam feest (met als hoogtepunt natuurlijk de partybus)! Dank voor deze onvergetelijke tijd en de leuke momenten die we nog steeds samen hebben!

RowZeetjes, Sanne, Mechteld, Ivette, Heleen, Marlies, Liselotte en Nathalie, na het afronden van onze studies zagen we elkaar iets minder frequent, maar gelukkig hebben we nog altijd contact. De gezamenlijke weekendjes/dagjes en feestjes, de appjes over alles en niets, en het feit dat ik weet dat ik jullie altijd om raad kan vragen, maakt onze vriendschap voor mij heel bijzonder. Sanne en Mechteld, jullie wil ik graag nog speciaal bedanken: De stapavondjes, carnaval, uitstapjes in binnen- en buitenland en het jaarlijks etentje bij mijn ouders zijn voor mij heel waardevol! 
Karin en Koos, een vermelding in mijn dankwoord is wel het minste wat ik terug kan doen. Dank voor al jullie enthousiasme bij in het werven van proefpersonen, jullie gezelligheid en de heerlijke etentjes.

Lin, het is niet zo toevallig dat wij steeds weer een powerkoppel zijn. Vanaf onze eerste kennismaking weten we elkaar perfect aan te vullen; jij lief lachend, ik magneten schietend. Dank voor alle interessante discussies, gezellige lunches en leuke (verkleed)feestjes!

Eric, ik kan me niet voorstellen hoe het is zonder een goede vriend waarmee je zoveel uiteenlopende dingen kunt delen; van afzien op een op een Kroatische berg tot culinaire hoogstandjes. Bedankt!

Tim, ondanks de fysieke afstand en twee totaal verschillende karakters, hebben we bijna dagelijks contact waarbij we (tussen alle grappen door) klein en groot nieuws bespreken en sportwedstrijden van het gepast en ongepast commentaar voorzien. Ik realiseer me dat ik het volgende niet altijd voldoende heb laten blijken, maar jij bent voor mij hét voorbeeld dat het maken van je eigen, soms onconventionele, keuzes tot heel veel mooie kansen en ervaringen kunnen leiden.

Frans en Ellie, papa en mama, hoe meer ik leer en meemaak, hoe meer ik besef dat aan alles wat ik kan en mag doen een enorm sterk fundament ten grondslag ligt. Dank voor al jullie hulp, betrokkenheid, ruimdenkendheid én humor! 


\section{Curriculum Vitae}

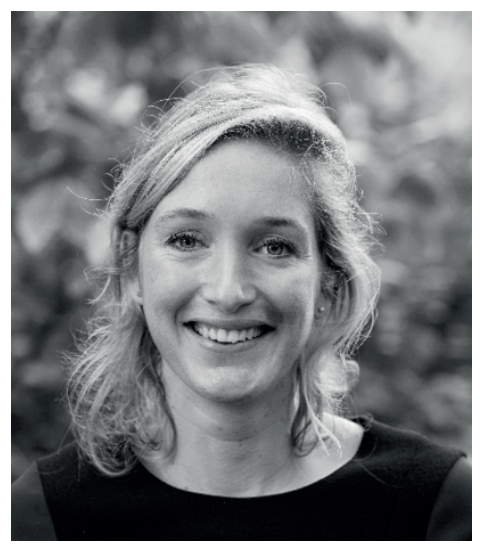

Noreen van der Linden was born on October 20th, 1987 in Breda, the Netherlands. After completing her secondary school with the distinction cum laude at Stedelijk Gymnasium Breda (Breda, the Netherlands) in 2006, she studied Molecular Life Sciences at Maastricht University (Maastricht, the Netherlands). After obtaining her Bachelor's degree, she enrolled in the 4-year Physician-Clinical Investigator Research Master at the same university. During her medical training, she was awarded the top 3\% student award. In 2013 she graduated with the distinction cum laude. After her graduation, Noreen started her PhD-program under supervision of prof. dr. Marja van Dieijen-Visser, prof. dr. Luc van Loon and dr. Steven Meex at the department of Clinical Chemistry of the Central Diagnostic Laboratory of the Maastricht University Medical Center+ (MUMC+) (Maastricht, the Netherlands). As part of her PhD-program, she visited the Cardiovascular Research Institute Basel (CRIB) at Basel University Hospital (Basel, Switzerland) (supervised by prof. dr. Christian Mueller).

During the first two years of her PhD-program, Noreen was a board member of the AAVMUMC+. In 2015, she interrupted her PhD-program for four months to take part in the National ThinkTank. In a team of twenty-four (PhD-)students, she examined the educational system in the Netherlands and presented an advice on future improvement. Afterwards, she was involved in various ThinkTank projects on topics like e-Health and intergenerational processes. During the academic year 2016-2017, she joined the KNAW Academy Honors Program for Young Artists and Scientists.

The scientific work in this thesis was published in several peer-reviewed journals and presented on national and international conferences. In addition, Noreen won the Dutch Society of Clinical Chemistry Abstract Price, was nominated for the Pélerin Award and received a travel grant from the European Society of Cardiology.

Noreen will pursue her career in healthcare strategy consulting at Gupta Strategists. 


\section{List of Publications}

Streng AS*, van der Linden N*, Kocken JM, Bekers O, Bouwman FG, van Dieijen-Visser MP, Mariman EC, Meex SJ, Wodzig WKH, de Boer D. Mass spectrometric identification of cardiac troponin T in urine of patients suffering from acute myocardial infarction. J Appl Lab Med.

van der Linden N, Hilderink JM, Cornelis T, Kimenai DM, Klinkenberg $\amalg$, van Doorn WP, Litjens EJ, van Suijlen JD, van Loon LJ, Bekers O, Kooman JP, Meex SJ. Twenty-four hour biological variation profiles of cardiac troponin I in subjects with or without chronic kidney disease. Clin Chem. 2017 Oct;63(10):16551656.

van der Linden N, Cornelis T, Kimenai DM, Klinkenberg $\sqcup$, Hilderink JM, Lück S, Litjens EJ, Peeters FE, Streng AS, Breidthardt T, van Loon L, Bekers O, Kooman JP, Westermark PO, Mueller C, Meex SJ. The origin of cardiac troponin T levels in chronic kidney disease. Circulation. 2017 Sep 12;136(11):10731075.

van der Linden N*, Streng AS*, Bekers O, Wodzig WKH, Meex SJ, de Boer D. Large variation in measured cardiac troponin $\mathrm{T}$ concentrations after standard addition in serum or plasma of different individuals. Clin Chem. 2017 Jul;63(7):1300-1302.

Hilderink JM, Klinkenberg $\amalg$, Aakre KM, de Wit NC, Henskens YM, van der Linden N, Bekers O, Rennenberg RJ, Koopmans RP, Meex SJ. Within-day biological variation and (hour to hour) reference change values for hematological parameters. Clin Chem Lab Med. 2017 Jun 27;55(7):1013-1024.

van der Linden N, Streng AS, Wodzig WK, Bekers O, Meex SJ. Better, higher, lower, faster: Increasingly rapid clinical decision making using high-sensitivity cardiac troponin assays. J Lab Precis Med 2017;2:14.

van der Linden N, Klinkenberg LJ, Bekers O, van Loon LJ, van Dieijen-Visser MP, Zeegers MP, Meex SJ. Prognostic value of basal high-sensitive cardiac troponin levels on mortality in the general population: A systematic review and meta-analysis. Medicine. 2016 Dec;95(52):e5703.

Peeters FE, van der Linden N, Thomassen AL, Crijns HJ, Meex SJ, Kietselaer BL, Crijns HJ. Clinical and echocardiographic determinants in bicuspid aortic dilatation. Medicine. 2016 Dec;95(52):e5699.

Klinkenberg $L^{*}$, Wildi $K^{*}$, van der Linden N, Kouw IW, Niens M, Twerenbold R, Rubini Gimenez M, Puelacher C, Neuhaus JD, Hillinger P, Nestelberger T, Boeddinghaus J, Grimm K, van Suijlen JD, Tan FE, ten Kate J, Bekers O, van Loon LJ, van Dieijen-Visser MP, Mueller C, Meex SJ. A diurnal rhythm of cardiac troponin: consequences for the diagnosis of acute myocardial infarction. Clin Chem. 2016 Dec;62(12):1602-1611. 
Klinkenberg $L$, Luyten P, van der Linden N, Urgel K, Snijders DP, Knackstedt C, Dennert R, Kietselaer BL, Mingels AM, Cardinaels EP, Peeters FE, van Suijlen JD, Ten Kate J, Marsch E, Theelen TL, Sluimer JC, Wouters K, Bekers O, Bekkers SC, van Loon LJ, van Dieijen-Visser MP, Meex SJ. Cardiac Troponin T and I Release After a 30-km Run. Am J Cardiol. 2016 Jul 15;118(2):281-7.

van der Linden N, Cornelis T, Klinkenberg L, Kimenai DM, Hilderink JM, Litjens EJ, Kooman JP, Bekers O, van Dieijen-Visser MP, Meex SJ. Strong diurnal rhythm of troponin T, but not troponin I, in a patient with renal dysfunction. Int J Cardiol. 2016 Jun 28;221:287-288.

Muris AH, Smolders J, Rolf L, Klinkenberg $L$, van der Linden N, Meex S, Damoiseaux J, Hupperts R. Vitamin D Status Does Not Affect Disability Progression of Patients with Multiple Sclerosis over Three Year Follow-Up. PLoS One. 2016 Jun 8;11(6):e0156122.

van der Linden $N$, Klinkenberg $L$, Leenders $M$, Tieland $M$, Verdijk LB, Niens $M$, van Suijlen JD, de Groot LC, Bekers O, van Loon $L$, van Dieijen-Visser MP, Meex SJ. The effect of exercise training on the course of cardiac troponin T and I levels: three independent training studies. Sci Rep. 2015 Dec 16;5:18320.

van der Linden N, Klinkenberg L, Meex SJ, Beckers EA, de Wit NC, Prinzen L. Immature platelet fraction measured on the Sysmex XN hemocytometer predicts thrombopoietic recovery after autologous stem cell transplantation. Eur J Haematol. 2014 Aug;93(2):150-6.

van der Linden $\mathrm{N}^{*}$, Tieland $\mathrm{M}^{*}$, Klinkenberg $L$, Verdijk LB, de Groot LC, van Loon LJ, van Dieijen-Visser MP, Meex SJ. The effect of a six-month resistance-type exercise training program on the course of high sensitive cardiac troponin T levels in (pre)frail elderly. Int J Cardiol. 2014 Aug 1;175(2):374-5.

van der Linden $\mathrm{N}^{*}$, Wildi K, Twerenbold R, Pickering J, Than M, Cullen L, Greenslade J, Parsonage W, Nestelberger T, Boeddinghaus J, Badertscher P, Rubini Giménez M, Klinkenberg $L$, Bekers O, Schöni A, Keller DI, Sabti Z, Puelacher C, Cupa J, Schumacher L, Kozhuharov N, Grimm K, Shrestha S, Flores Widmer D, Michael M, Stelzig C, Strebel I, Miró O, Rentsch K, Morawiec B, Kawecki D, Kloos W, Lohrmann J, Richards AM, Troughton R, Pemberton C, Osswald S, van Dieijen-Visser MP, Mingels AM, Reichlin T, Meex SJ, Mueller C. Combining Cardiac Troponin I and Cardiac Troponin T in the Early Diagnosis of Acute Myocardial Infarction. Under revision.

Hilderink JM, van der Linden N, Klinkenberg $L$, Kimenai DM, Mohammed Aref B, Aziz F, Rennenberg RJ, Bekers O, Koopmans RP, Meex SJ. Twenty-four hour biological variation of eGFR in subjects with or without chronic kidney disease. Under review.

Nationale DenkTank 2015. LeerWijzer. ISBN: 978-90-78757-08-5.

* Equal contribution 